\title{
INVESTIGATION OF VEHICLE TO \\ VEHICLE COMMUNICATION SYSTEM \\ USING VISIBLE LIGHT TECHNOLOGY \\ by
}

Hasan Farahneh

Master of Science, The University of Jordan, 2004

Bachelor of Engineering, Al-Yarmouk University, 1985

A dissertation

presented to Ryerson University

in partial fulfillment of the

requirements for the degree of

Doctor of Philosophy

in the Program of

Electrical and Computer Engineering

Toronto, Ontario, Canada, 2018

(c)Hasan Farahneh 2018 
AUTHOR'S DECLARATION FOR ELECTRONIC SUBMISSION OF A DISSERTATION

I hereby declare that I am the sole author of this dissertation. This is a true copy of the thesis, including any required final revisions, as accepted by my examiners.

I authorize Ryerson University to lend this dissertation to other institutions or individuals for the purpose of scholarly research.

I further authorize Ryerson University to reproduce this dissertation by photocopying or by other means, in total or in part, at the request of other institutions or individuals for the purpose of scholarly research.

I understand that my dissertation may be made electronically available to the public. 
Investigation of Vehicle to Vehicle Communication System Using Visible Light Technology

Doctor of Philosophy 2018

Hasan Farahneh

Electrical and Computer Engineering

Ryerson University

\begin{abstract}
Visible Light Communication (VLC) has emerged as an attractive alternative to radio frequency $(\mathrm{RF})$ communication, due to cost-effectiveness and being license free. It has also proved its applicability in automotive applications, as an alternative and/or a complement to the traditional RF-based communications. We investigate the suitability of VLC in Intelligent Transportation Systems (ITS) and discuss its advantages in terms of safety enhancement and improved efficiency of the ITS. In this thesis, a VLC-based Vehicle-to-Vehicle (V2V) system in practical environments, considering both Line-of-Sight (LOS) and Non-Line-of-Sight (NLOS) paths are presented. The thesis investigates robust communication between a Light Emitting Diodes (LEDs) based VLC emitter and Photodiodes (PDs) based VLC receiver. For consideration of a V2V communication system, we consider transmitter on vehicle headlights and receivers on taillight making $2 \times 2$ Multiple-Input-Multiple-Output (MIMO) communication link. A closed-form expression of the Channel Impulse Response (CIR) is derived and the effect of various channel parameters is analyzed. Optical-Orthogonal Frequency Division Multiplexing (O-OFDM) with adaptive modulation schemes is proposed for system improvement. Its performance is evaluated in terms of Inter Symbol Interference (ISI) mitigation, and data rate improvement. Moreover, the effect of sunlight on the V2V-VLC system is investigated with appropriate denoising schemes. Two denoising schemes are proposed and evaluated as a solution to combat the effect of the solar irradiance on the VLC signal. Firstly, we use a differential receiver for denoising purposes followed by k-Nearest Neighbour (kNN) based adaptive filtering algorithm, which is a supervised Machine Learning (MLE) technique. The shadowing effect is also studied. Moreover, an application of VLC in
\end{abstract}


Foglet based ITS is described. The simulation validation of the VLC-based V2V system is performed under various environmental conditions and scenarios. Obtained results emphasize the suitability of VLC technology for automobile applications. 


\section{Acknowledgment}

All praises are due to Almightily Allah, the most Gracious, and the most Merciful. I thank Him for all the bounties and blessings He bestowed upon me and for granting me the strength and patience to complete this work.

I would like to express my sincerest gratitude to my supervisor, Professor Xavier Fernando, for his patient guidance, warm encouragement and invaluable advice throughout the course of my $\mathrm{PhD}$ study. He was always understanding of the difficulties and the challenges one inevitably faces during the seemingly never ending $\mathrm{PhD}$ study. His positive outlook and endless support inspired me and gave me the confidence and motivation needed to finish this work. His careful and insightful reviews contributed enormously to the production of this thesis. It was truly a great privilege working with him as I learnt from him a lot that will pave the road in my future.

I would like to thank my thesis committee members, chairs and professors, for taking the time and effort to review my thesis and provide me with their insightful and valuable comments. I would also like to acknowledge the administrative staff at the school of graduate studies and the department of Electrical and Computer Engineering at Ryerson University for all the instances in which their assistance helped me along the way. Finally, I would like to thank my colleagues at Ryerson Communication Lab (RCL) and research group present and past, I am lucky to be a part of this group where a team spirit truly prevails. Our group discussions and their insightful feedback have helped in many occasions. 


\section{Table of Contents}

Declaration ............................ ii

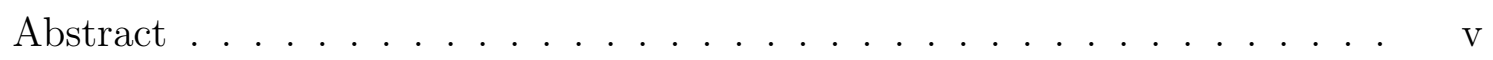

Acknowledgment ........................ vi

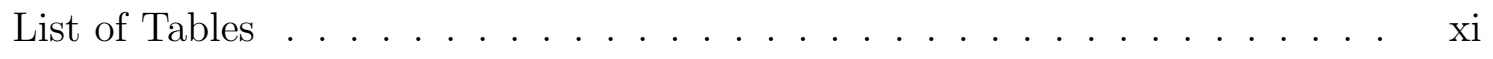

List of Figures . . . . . . . . . . . . . . . xiv

List of Acronyms ..................... $\ldots \ldots$

1 Introduction 1

1.1 Background . . . . . . . . . . . . . . . . . . . . 1

1.2 The Architecture of VLC System . . . . . . . . . . . . . . 3

$1.2 .1 \quad$ VLC Emitter . . . . . . . . . . . . . . . . . . 3

$1.2 .2 \quad$ VLC Receiver . . . . . . . . . . . . . . . . . . 4

$1.2 .3 \quad$ VLC Channel . . . . . . . . . . . . . . . . . . . 5

1.2.4 Visible Light Terminology . . . . . . . . . . . . . . . . . 5

1.3 Channel Noise and SNR . . . . . . . . . . . . . . . . . . 7

1.4 Comparison of Radio Frequency and Visible Light Propagation Channels . . 9

1.4.1 VLC Advantages and Drawbacks . . . . . . . . . . . . 10

1.5 Visible Light Communications in Automotive

Applications . . . . . . . . . . . . . . . . . . . 11

1.5 .1 Usage of VLC in ITS . . . . . . . . . . . . . . . . . 12

1.5.2 VLC in the ITS / State of the Art . . . . . . . . . . 13 
1.6 VLC Research Direction and Future Challenges . . . . . . . . . . . . 16

1.7 Motivation of the Work . . . . . . . . . . . . . . . . . . 17

1.8 Contribution . . . . . . . . . . . . . . . . . . . . . . . . . . 18

1.9 Thesis Outline . . . . . . . . . . . . . . . . . . . . . . . . . . . . . . . . . . . . . . . 19

1.10 Chapter Summary . . . . . . . . . . . . . . . . . . . . . 21

2 Multipath Channel Model for V2V-VLC System 22

2.1 Related Work in Channel Modeling for VLC . . . . . . . . . . . . . . . . . . 23

$2.2(2 \times 2)$ MIMO V2V-VLC System Model . . . . . . . . . . . . 25

2.2.1 The V2V-VLC System Considerations . . . . . . . . . . . . 25

2.2 .2 System Model . . . . . . . . . . . . . . . . . . . . . . . . . 27

2.3 Channel Impulse Response and Transfer Function . . . . . . . . . . . . . . 29

2.3.1 Impulse Response and Transfer Function of the LOS Component . . . 29

2.3.2 Impulse Response and Transfer Function of the NLOS Component . . 31

2.3.3 Derivation of NLOS Transfer Function . . . . . . . . . . . . . . . 34

2.4 Performance Analysis of the V2V-VLC Channel Model . . . . . . . . . . . . 37

2.4.1 The Received Optical Power and SNR . . . . . . . . . . . . 37

2.4 .2 Calculating the BER . . . . . . . . . . . . . . . . . . . . . . . 39

2.4.3 Time Dispersion Parameters for the Channel . . . . . . . . . . . . . . 40

2.5 Simulation and Results . . . . . . . . . . . . . . . . . . . . . . . 42

2.6 Chapter Summary . . . . . . . . . . . . . . . . . . . . . . . 50

3 Performance Analysis of Adaptive OFDM Modulation Scheme in VLC $\begin{array}{ll}\text { Vehicular Communication Network } & 51\end{array}$

3.1 Related Work in Adaptive Modulation for VLC . . . . . . . . . . . . 52

3.2 Optical OFDM Scheme of V2V-VLC System _ . . . . . . . . . . 53

3.2 .1 DCO-OFDM Modulation Scheme . . . . . . . . . . . . 53

$3.2 .2 \quad$ ACO-OFDM Modulation Scheme . . . . . . . . . . . . . . 58

3.3 Performance Analysis and Bit Loading Algorithm . . . . . . . . . . . . 60 
3.3.1 SNR Estimation of Subcarriers Using SVD . . . . . . . . . . . . . 60

3.3.2 Adaptive Transmission and Bit Loading Scheme . . . . . . . . . . 62

3.4 Results and Discussions _... . . . . . . . . . . . . . 63

3.4 .1 Simulation Environment . . . . . . . . . . . . . . . . . 63

3.4 .2 Results and Analysis . . . . . . . . . . . . . . . . . . . . . 64

3.5 Chapter Summary . . . . . . . . . . . . . . . . . . . . 70

4 Proposed Precoder and Equalizer in $2 \times 2$ MIMO VLC Systems for Vehicular $\begin{array}{ll}\text { Application } & 71\end{array}$

4.1 Related Work in Precoding and Equalization for VLC . . . . . . . . . . . 72

$4.2 \quad$ System Model . . . . . . . . . . . . . . . . . . . . . . . . 73

4.2 .1 Investigating the Transmitter $\ldots \ldots \ldots \ldots \ldots$

4.2 .2 Investigating the Receiver $\ldots \ldots \ldots \ldots \ldots$

$4.2 .3 \quad$ Equalization Matrix Design . . . . . . . . . . . . . . . . 78

4.2 .4 Precoding Matrix . . . . . . . . . . . . . . . . 81

4.3 Simulation and Results . . . . . . . . . . . . . . . . . . . . 82

4.4 Chapter Summary . . . . . . . . . . . . . . . . . . . . . . 84

5 Shadowing Effects on V2V-VLC 85

5.1 Channel Impulse Response with Shadowing . . . . . . . . . . . . . . . 86

5.2 Shadowing Effect $\ldots \ldots \ldots \ldots \ldots \ldots$

5.3 Error Probability . . . . . . . . . . . . . . . . . . . . 90

5.4 Simulations . . . . . . . . . . . . . . . . . . . . . 95

5.5 Chapter Summary . . . . . . . . . . . . . . . . . . . . . . . 97

6 Sunlight Effect on V2V-VLC System and Denoising Schemes 98

6.1 Solar Irradiance . . . . . . . . . . . . . . . . . . . . . . . . . . . . . . . . . 99

6.2 SNR of V2V-VLC System . . . . . . . . . . . . . . . 100

6.3 Related Work in Denoising Schemes for VLC . . . . . . . . . . . . . 103 
6.4 Noise Calculations . . . . . . . . . . . . . . . . . . . . . . . . . . 104

6.4 .1 Other Noise Sources _. . . . . . . . . . . . . . . . . . . 105

6.4 .2 Performance Metrics . . . . . . . . . . . . . . . . . . . 106

6.5 Differential Receiver as a Denoising Scheme to Improve the Performance of V2V-VLC Systems . . . . . . . . . . . . . . . . 106

6.5.1 Differential Filtering Scheme for $2 \times 2$ MIMO-V2V-VLC . . . . . 107

6.5.2 Signal Filtration by Differential Receiver . . . . . . . . . . . . . 109

6.5.3 Simulation Results . . . . . . . . . . . . . . . . . . . . . . . 112

6.6 Denoising Scheme to Improve the Performance of V2V-VLC Systems by

Machine Learning . . . . . . . . . . . . . . . . . . . . . . . . . 114

6.6 .1 System Model . . . . . . . . . . . . . . . . . . . . . . . . 115

6.6.2 Machine Learning and Adaptive Filtering . . . . . . . . . . . . 115

6.6 .3 Why kNN Algorithm . . . . . . . . . . . . . . . . . . . 115

6.6.4 Problem Classification . . . . . . . . . . . . . . . . 116

6.6 .5 Adaptive Filtering . . . . . . . . . . . . . . . . . 117

6.6.6 k-Nearest Neighbour Algorithm and Distance weighted kNN rule . . . 117

6.6 .7 Simulation Results . . . . . . . . . . . . . . . . . . . 120

6.6 .8 Execution Time . . . . . . . . . . . . . . . . . . . . . . . 123

6.7 Chapter Summary . . . . . . . . . . . . . . . . . . . . . 125

7 Applications on the Proposed Channel Model $\quad 126$

7.1 VLC Enabled Foglets Assisted Road Asset Reporting . . . . . . . . . . . 126

$7.2 \quad$ VLC Enabled Foglets . . . . . . . . . . . . . . . . . . . 127

7.2 .1 Fog Computing . . . . . . . . . . . . . . . . . . . . 128

7.2 .2 Challenges and Motivation . . . . . . . . . . . . . . . . . 129

7.2.3 Foglets and Distributed Data Processing . . . . . . . . . . . . . 129

7.2 .4 Why VLC for Foglets . . . . . . . . . . . . . . . . . . 130

7.3 Road Asset Management and Incident Reporting . . . . . . . . . . . 130 
7.3.1 Infrastructure . . . . . . . . . . . . . . . . . . 131

7.3.2 Smart Vehicle . . . . . . . . . . . . . . . . . 132

7.3.3 Foglets Infrastructure . . . . . . . . . . . . . . . . . 132

7.3.4 Optical Communication Sensors . . . . . . . . . . . . 133

7.3.5 Foglets Reporting . . . . . . . . . . . . . . . . . . 134

7.4 Investigate VLC Channel between Vehicle and Foglets . . . . . . . . . . . . . 134

7.4.1 BER of VLC Link . . . . . . . . . . . . . . . . . 135

7.4.2 Results and Discussion . . . . . . . . . . . . . . 135

7.5 Chapter Summary . . . . . . . . . . . . . . . . . . 139

8 Discussion and Future Work $\quad 140$

8.1 Conclusions . . . . . . . . . . . . . . . . . . . . . . 141

8.2 Future Developments and Perspectives . . . . . . . . . . . . . . . 145

$\begin{array}{lr}\text { Bibliography } & 147\end{array}$ 


\section{List of Tables}

1.1 Probability Density Function (PDF)s of different types of noise . . . . . . . 9

1.2 Noise parameters and their simulation values . . . . . . . . . . . . 9

1.3 Comparison of RF and VLC propagation channels for vehicular communications 10

2.1 Simulation parameters . . . . . . . . . . . . . . . . 42

2.2 RMS delay spread comparison . . . . . . . . . . . . . . . 46

2.3 Median coherence time comparison (units in $(m s)$ ) . . . . . . . . . 47

3.1 Simulation parameters . . . . . . . . . . . . . . . 65

3.2 Look-Up-Table . . . . . . . . . . . . . . . . . . . 66

6.1 Solar irradiance at the city of Toronto [1] . . . . . . . . . . . . . . 101

6.2 Classes of natural light $[2] \ldots \ldots \ldots 1$ 


\section{List of Figures}

1.1 VLC transceiver block diagram. . . . . . . . . . . . . . . . 3

1.2 The relationship between the illuminance and the candela $[3] \ldots \ldots$

1.3 Distribution of the electromagnetic spectrum [4] . . . . . . . . . . . 11

1.4 Application scenario for vehicular-VLC based [5]. . . . . . . . . . . . . 12

1.5 Integration of LEDs lighting systems in series vehicles [6] . . . . . . . . . 14

1.6 Summary of the thesis outline. . . . . . . . . . . . . . . . . 20

$2.1(2 \times 2)$ MIMO model for V2V-VLC showing the LOS and NLOS paths. . . 25

2.2 Headlight optical radiation pattern $[7] \ldots \ldots \ldots$

2.3 Block diagram of a $2 \times 2$ MIMO link of V2V-VLC system $\ldots \ldots \ldots$

2.4 Emitting and incident angles and other related parameters. . . . . . . . . 30

2.5 Illustration of how the NLOS path's matrices ( $\mathbf{A}, \mathbf{B}$, and $\mathbf{C}$ ) are constructed. 32

2.6 Conditional probability density function with OOK modulation . . . . . . . 39

2.7 Channel impulse response of the LOS and NLOS components when vehicles are moving in the same direction. . . . . . . . . . . . . . . 43

2.8 The LOS and NLOS paths transfer functions. . . . . . . . . . . . . 44

2.9 Comparison between derived NLOS CIR and NLOS CIR given by EQ (2.23). 44

2.10 Received power versus the distance between vehicles. . . . . . . . . . . . 45

2.11 BER versus the distance between vehicles moving in same direction. . . . . . 46

2.12 RMS delay spread for one reflection system. . . . . . . . . . . . . . . 47

2.13 Channel coherence time when both vehicles move in the same direction. . . . 48 
2.14 Channel coherence time when both vehicles move in the opposite direction. . 48

2.15 Coherence bandwidth for one reflection system with 0.9 correlation level when vehicles move in same and opposite direction. . . . . . . . . . . . . . . 49

3.1 Simplified diagram of the V2V-VLC-OFDM scheme. . . . . . . . . . . . 54

3.2 (a) OFDM bipolar signal (b) The signal after adding DC bias voltage (c) The signal after clipping the remaining negative. . . . . . . . . . . . 56

$3.3 \mathrm{I}-\mathrm{V}$ characteristic curve of the LED. . . . . . . . . . . . . . . . . . . . . 64

3.4 Number of loaded bits for DCO-OFDM when $I_{T}\left(\alpha_{T}, \beta_{T}\right)=1500(c d)$. . . 66

3.5 Number of loaded bits for ACO-OFDM when $I_{T}\left(\alpha_{T}, \beta_{T}\right)=1500(c d) . \quad$. . 67

3.6 The transmission rate of DCO-OFDM with adaptive modulation. . . . . . . 68

3.7 The transmission rate of ACO-OFDM with adaptive modulation. . . . . . 68

3.8 SNR vs. BER for ACO-OFDM with FFT size=64. . . . . . . . . . . . . 69

3.9 SNR vs. BER for DCO-OFDM with FFT size=64. . . . . . . . . . . . . . 70

4.1 Illustration of the investigated precoder and equalizer system. . . . . . . . 73

4.2 Comparison of the suggested detection algorithms in terms of BER. . . . . . 83

4.3 Comparison of the suggested detection algorithms in terms of MSE. . . . . . 84

5.1 Outdoor shadowing . . . . . . . . . . . . . . 86

5.2 Bimodal function with equal variances . . . . . . . . . . . . . 89

5.3 The PDF of no shadowing . . . . . . . . . . . . . . . . . . . 91

5.4 The PDF of moderate shadowing . . . . . . . . . . . . . . 91

5.5 The PDF of severe shadowing . . . . . . . . . . . . . . . . 92

5.6 Error probability with different shadowing effects _ . . . . . . . . . . 96

6.1 Sunlight spectrum $[8] \ldots \ldots \ldots 9$

6.2 The effect of solar irradiance on V2V-VLC . . . . . . . . . . . . . . 100

6.3 Average predicted solar irradiance at the city of Toronto in month of June [1]. 102 
6.4 SNR without any filtration scheme at the city of Toronto when the LED illuminance intensity is $1750(c d)[1] \ldots \ldots$. . . . . . . . . . 102

6.5 The receiver architecture of the VLC system. . . . . . . . . . . . . 107

6.6 Denoising differential scheme of VLC . . . . . . . . . . . . . . . . . . . . 108

6.7 Background noise PSD . . . . . . . . . . . . . . . . . . . . . . . . 111

6.8 Average predicted solar irradiance at Toronto city in month of June with the denoising schemes. . . . . . . . . . . . . . . . . . . . . 113

6.9 BER with sunlight irradiance considering city of Toronto. . . . . . . . . . . 113

6.10 Achievable data rate with sunlight irradiance considering city of Toronto. . . 114

6.11 Adaptive filtering using $\mathrm{kNN}$ algorithm $\ldots \ldots \ldots \ldots$

6.12 Training data and observed data in Voronoi diagram. . . . . . . . . . . . . 121

6.13 The relation between the number of neighbors and the percentage of decision error. . . . . . . . . . . . . . . . . . . . . 122

6.14 BER of the V2V-VLC system with and without denoising scheme. . . . . . 123

6.15 Probability of each action with day hours. . . . . . . . . . . . . . . 124

6.16 Execution time for the proposed scheme. . . . . . . . . . . . . . . . . . . 124

7.1 VLC Assisted asset management infrastructure. . . . . . . . . . . . . . . 131

7.2 Foglets architecture. . . . . . . . . . . . . . . . . . . . . . 132

7.3 Sequence of Foglets tasks . . . . . . . . . . . . . . . . . . . . . 133

7.4 Isocandela diagrams of the road surface from a pair of low beam and high beam headlamp $[7] \ldots \ldots \ldots \ldots$

7.5 Received power vs the distance between the vehicle and the Foglet station. . 138

7.6 The BER vs the LED illuminance intensity . . . . . . . . . . . . . . . 138 


\section{List of Acronyms}

ACO-OFDM Asymmetrically Clipped Optical Orthogonal Frequency Division Multiplexing
$\mathrm{A} / \mathrm{D}$
Analog-to-Digital
APD
Avalanche Photodiode
AWGN Additive White Gaussian Noise
BER Bit Error Rate
BPF Band Pass Filter
BPSK Binary Phase Shift Keying
BRDF Bidirectional Reflectance Distribution Function
CIR Channel Impulse Response
CP Cyclic Prefix
CMOS Complementary Metal-Oxide Semiconductor
CSI Channel State Information
D/A Digital-to-Analog

DCO-OFDM Direct Current Optical Orthogonal Frequency Division Multiplexing

DMT Discrete Multi-Tone Modulation

DSSS Discrete Sequence Spread Modulation

FFT Fast Fourier Transform

FOV Filed of View

IFFT Inverse Fast Fourier Transform

IM/DD Intensity Modulation and Direct Detection

IoT Internet of Things

IR Infrared

ISI Inter Symbol Interference

ITS Intelligent Transportation Systems

IVC Inter-Vehicle Communication 


\begin{tabular}{ll} 
I2V & Infrastructure-to-Vehicle \\
kNN & k-Nearest Neighbour \\
LEDs & Light Emitting Diodes \\
LOS & Line-of-Sight \\
LPF & Low Pass Filter \\
MIMO & Multiple-Input-Multiple-Output \\
ML & Maximum Likelihood \\
MLE & Machine Learning \\
MMSE & Minimum Mean-Square Error \\
MSE & Mean Squared Error \\
NLOS & Non-Line-of-Sight \\
OCI & Optical Communication Image Sensor \\
OFDM & Orthogonal Frequency Division Multiplexing \\
O-OFDM & Optical-Orthogonal Frequency Division Multiplexing \\
OOK & ON-OFF-Keying \\
OWC & Optical Wireless Communication \\
PAM & Pulse Amplitude Modulation \\
PD & Photodiode \\
PDF & Probability Density Function \\
PDs & Photodiodes \\
PIN & Positive Intrinsic Negative Photodiodes \\
PPM & Pulse Position Modulation \\
PSD & Power Spectral Density \\
P/S & Parallel-to-Serial \\
QAM & Quadrature Amplitude Modulation \\
QoS & Quality of Service \\
RMS & Root Mean Squared \\
RSU & Road Side Units \\
\hline
\end{tabular}




$\begin{array}{ll}\text { RZ } & \text { Return to Zero } \\ \text { SISO } & \text { Single-Input Single-Output } \\ \text { SNR } & \text { Signal-to-Noise-Ratio } \\ \text { S/P } & \text { Serial-to-Parallel } \\ \text { STBC } & \text { Space-Time Block Coding } \\ \text { SVD } & \text { Singular Value Decomposition } \\ \text { TDE } & \text { Time Domain Equalization } \\ \text { VLC } & \text { Visible Light Communication } \\ \text { V2I } & \text { Vehicle-to-Infrastructure } \\ \text { V2V } & \text { Vehicle-to-Vehicle } \\ \text { WDM } & \text { Wave Division Multiplexing } \\ \text { ZF } & \text { Zero Forcing }\end{array}$




\title{
List of Symbols
}

\author{
$A_{r} \quad$ The effective area of the photo detector \\ $A_{q} \quad$ The area of the reflector $\mathrm{q}$ \\ $\alpha \quad$ The transmitter horizontal angle in relation to the transmitter axis \\ $\beta \quad$ The transmitter vertical angle in relation to the transmitter axis \\ $B_{n} \quad$ Equivalent noise bandwidth \\ c The speed of light \\ $\otimes \quad$ The convolution sign \\ $d \omega \quad$ The solid angle measured by steradian $(s r)$ \\ $d P_{r} \quad$ The received optical power from any single reflected \\ $\delta \quad$ The impulse function \\ $E_{i} \quad$ The irradiance incident onto the surface \\ $\eta \quad$ Dimming level \\ $F \quad$ Excess noise \\ $F_{n} \quad$ Noise figure of the photodiode \\ $g\left(\theta_{n m}\right) \quad$ The concentrator gain \\ G Photodiode gain \\ $\gamma \quad$ The receiver responsivity \\ $h_{i} \quad$ The hight of the photo detector \\ $h(t) \quad$ The channel impulse response \\ $I(\alpha, \beta) \quad$ luminous intensity measured by candela $(c d)$ \\ $I_{o} \quad$ photo current produced due to the received optical power \\ $I_{s} \quad$ photo current produced due to sunlight \\ $I_{b g} \quad$ Receiver background noise current \\ $I_{d g} \quad$ Bulk dark current \\ $I_{d s} \quad$ Surface dark current \\ $I_{p} \quad$ The average photocurrent generated at the receiver
}




\begin{tabular}{|c|c|}
\hline$K_{B}$ & Boltzmanns constant \\
\hline$l$ & The Lambertian order of the source \\
\hline$L_{r}$ & The irradiance scattering from the surface \\
\hline$L_{\Phi}$ & The illuminance \\
\hline$\lambda$ & light wavelength \\
\hline$\mu_{0,1}$ & The mean values of the Gaussian function \\
\hline$n(t)$ & The noise signal \\
\hline$P_{e}$ & Probability of error \\
\hline$\Phi$ & Luminance flux \\
\hline$\phi_{n m}$ & The emitting angle from source $m$ to receiver $n$ \\
\hline$\phi_{\text {inc }}$ & The incident azimuth angle \\
\hline$\phi_{\text {ref }}$ & The reflected azimuth angle \\
\hline$P_{i n c}$ & The incident optical power \\
\hline$P_{r}$ & The received optical power \\
\hline$P_{T}$ & The total power \\
\hline$\Psi$ & The field of view of the receiver \\
\hline$q_{e}$ & Electron charge \\
\hline$R_{L}$ & Load resistance \\
\hline$\rho$ & The reflection coefficient of the reflector \\
\hline$R(\phi)$ & The reflected radiant intensity \\
\hline$R_{n m}$ & The distance between source $m$ and receiver $n$ \\
\hline$\theta_{n m}$ & The incident angle from source $m$ to receiver $n$ \\
\hline$\theta_{i n c}$ & The incident polar angle \\
\hline$\theta_{\text {ref }}$ & The reflected polar angle \\
\hline$\sigma^{2}$ & The variance of the signal \\
\hline$T_{k}$ & Temperature in Kelvin \\
\hline$\left.\theta_{n m}\right)$ & The signal transmission coefficient of an optic \\
\hline
\end{tabular}


$\tau \quad$ The time delay

$\zeta \quad$ Biasing ratio 


\section{Chapter 1}

\section{Introduction}

\section{$1.1 \quad$ Background}

Optical Wireless Communication (OWC), includes Infrared (IR) and visible light, is used to transmit information wirelessly. Visible Light Communication (VLC) is an emerging wireless communication technology which uses the visible light for dual purposes, illumination, and transmission of the data. Therefore, simple low-cost Intensity Modulation and Direct Detection (IM/DD) techniques are widely employed. This means that only the signal intensity is modulated and there is no phase information. IM/DD requires real-valued and unipolar electrical signal.

At the emitter side, the message is modulated onto the instantaneous power of the light, while at the receiver, a Photodiode (PD) is used to detect the variations of the light intensity and then, extract the transmitted data. The main advantage of VLC is the usage of the existing LEDs lighting systems which makes it ubiquitous and significantly reduces its implementation cost.

The RF-based communications begin to show their limitations. The limited availability of the spectrum and the increasing number of nodes affect the performances and the reliability of the link. Under these circumstances, it is obvious that a new wireless communication technology is required. VLC will be an attractive alternative to RF communication. In 
addition to its ubiquitous character, VLC offers a huge bandwidth available free of charge, enabling high data rate communications.

Wireless communication systems are very essential in transportation, especially in automotive field. By using wireless communication, safety messages can be transmitted from the traffic infrastructure to the approaching vehicles and from one vehicle to another. Furthermore, vehicles may share data concerning their state such as location, velocity, road conditions and many other concerns. Moreover, communications among the vehicles increase driver's awareness and enable the vehicle active safety systems. Also, it increases the efficiency of the transportation system by providing location services and optimized alternative routes.

Since the LEDs lighting began to appear in transportation in many aspects such as integrated with traffic lights, street lighting and traffic sings and in the vehicle lighting systems, VLC seems to be appropriate for providing wireless data exchange for automotive applications.

RF communication systems and VLC systems are fully compatible with each other, thus, VLC usage does not exclude RF communications. The benefit for using VLC in vehicular applications is well motivated for many reasons. Firstly, the ability of RF communications to support vehicular communication in high traffic densities and in crowded cities is rather questionable because of the mutual interferences. Secondly, VLC is a LOS technology, it is able to successfully support communications even in high traffic density.

To this end, VLC represents a new communication technology that uses energy efficient solid-state LEDs for both lighting and wireless data transmission. VLC uses the visible light $(380-780) \mathrm{THz}$ as a communication medium, which offers huge bandwidths free of charge, it is not limited by any law and it is safe to human body, allowing for high power transmissions. VLC has the potential to provide low-price high-speed wireless data communication. Even if VLC is a new technology, it had a fast development which is proof of its huge potential. 


\subsection{The Architecture of VLC System}

A VLC system consists of a transmitter to modulate the LEDs light and a receiver to predict the received light variation, then, extracting the data. Both, the transmitter and the receiver are connected through VLC channel. Figure. 1.1 illustrates the VLC system.

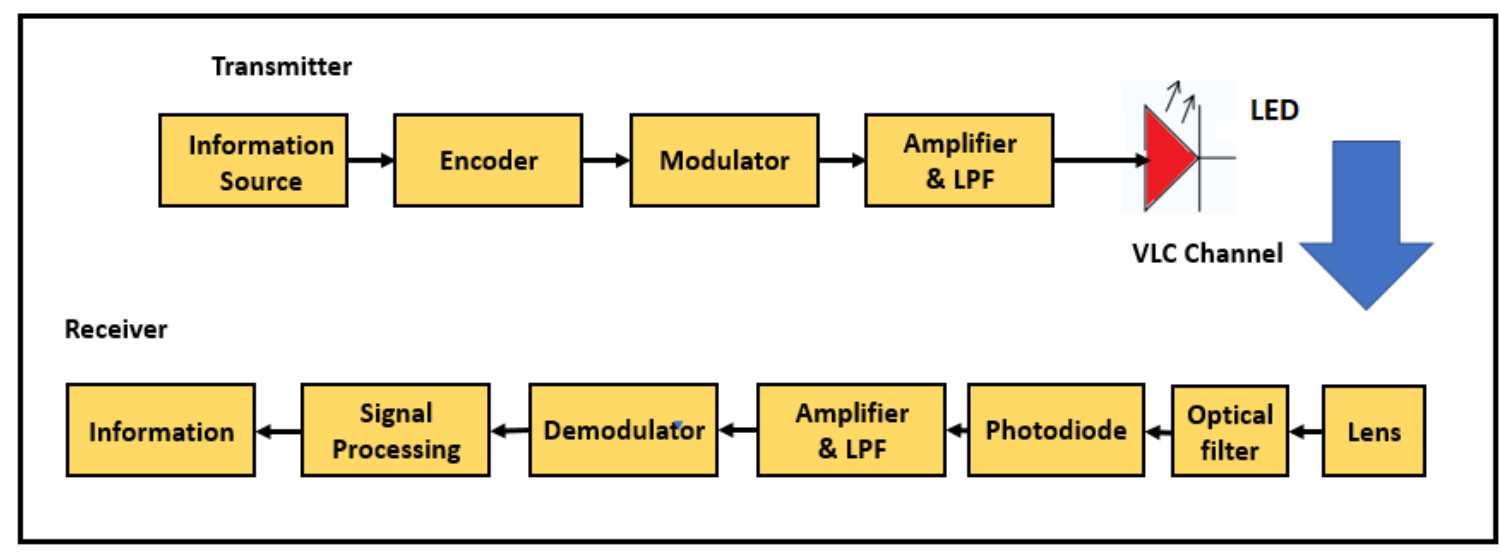

Figure 1.1: VLC transceiver block diagram.

\subsubsection{VLC Emitter}

A VLC emitter is a device that converts the binary data into messages that can be sent over the free space optical medium by using visible light. The VLC emitter has a dual purpose, emits light, and transmits data instantaneously by using the same optical power and without any noticeable flickering. The main component of the VLC emitter is the encoder which converts the data into a modulated message. The encoder controls the switching of the LEDs according to the transmitted binary data and the data rate, thus, converts the transmitted binary data into an amplitude modulated light beam. Generally, the light produced by the LEDs is current modulated with ON-OFF-Keying (OOK) amplitude modulation, but other modulation techniques such as Orthogonal Frequency Division Multiplexing (OFDM) [9], Discrete Multi-Tone Modulation (DMT) or Discrete Sequence Spread Modulation (DSSS) [10] can also be used. 
Basically, there are two types of emitters: R-G-B LED, and phosphorescent LED. The R-G-B LED consists of red, green, and blue chips and combines the three lights in a correct proportion to generate white light. R-G-B LED has relative higher modulation bandwidth $20 \mathrm{MHz}$ and can support Wave Division Multiplexing (WDM), but the cost is relatively high. Phosphorescent LED uses the blue LED chip coated with a yellow phosphor, which is the most popular white LED in the market due to its low cost. However, the slow response of phosphor limits the modulation bandwidth of the phosphorescent white LEDs to only few MHz. Optical filtering can be operated at the receiver to decrease the noise [11].

In general, the parameters of the VLC emitter are mainly limited by the characteristics of the LEDs. The data rate depends on the switching abilities of the LEDs while the emitters service area depends on the transmission power and on the illumination pattern. Currently, the solid-state lighting industry is able to produce LEDs that can offer switching frequencies of a few tens of $\mathrm{MHz}[4]$.

\subsubsection{VLC Receiver}

The VLC receiver is used to extract the data from the modulated light beam. It transforms the light into an electrical signal that will be demodulated and decoded by the embedded decoder module. There are two widely adopted types of PDs, ordinary Positive Intrinsic Negative Photodiodes (PIN) and Avalanche Photodiode (APD). APD is favored when there is little ambient-induced shot noise because their structure can overcome more preamplifier thermal noise.

The key parameter in PDs is the responsivity $\gamma$ which is defined as [12]

$$
\gamma=\frac{I_{p}}{P_{i n c}}
$$

where $I_{p}$ denotes the average photocurrent generated, and $P_{i n c}$ denotes the incident optical power. 
The PD responsivity depends on the physical structure of the PD and has the units of (A/W). The incident power $P_{i n c}$ is proportional to its effective light collection area.

Thus, the PD effective area must be large enough to collect enough transmitted signal. However, since the incident light is not only due to the emitter but also from other light sources

(artificial or natural), the receiver is subject to significant interferences. The performances of the VLC receiver can be enhanced using an optical filter that rejects the unwanted spectrum components.

In general, the VLC receiver must be selected such that the cost, performance, and safety requirements are satisfied [12].

\subsubsection{VLC Channel}

The transmitter and the receiver of the VLC system are connected through the free space optical communication channel. At the receiver, the received power intensity decreases with the square root of the distance between the transmitter and the receiver as it passes through the communication channel, making the received signal very low. Also, the VLC channel could contain many sources of optical noise. During the daytime, the most dominant source of the channel noise is the sun. Different noise sources and the weather condition make the VLC channel unpredictable. Rain, snow, dust particles or heavy fog can affect the VLC link by causing scattering of the light containing the data. Noise sources, together with the low signal power especially at a long distance, significantly affects the Signal-to-Noise-Ratio (SNR) in VLC systems. Another characteristic of the VLC channel comes from the strict of LOS conditions, which limits the multipath propagation. In VLC the multipath has a limited effect which is experienced only at short emitter-receiver distances.

\subsubsection{Visible Light Terminology}

In the following paragraphs, we will present some of the visible light terminologies to understand what the expressions "Lux" and "Lumens" mean and how they relate to the 
energy consumption and lighting levels.

The lux (symbol: $l x$ ) is the SI derived unit of illuminance and luminous emittance, measuring luminous flux per unit area. It is equal to one lumen per square meter. The $(l x)$ is converted to watts $/ \mathrm{m}^{2}$ for daylight by multiplying with (0.00402), only appropriate for the visible light band of interest [13].

The candela (symbol: $c d$ ) is the luminous intensity, in a given direction, of a source that emits monochromatic radiation of frequency $540 \times 10^{12} \mathrm{~Hz}$ and that has a radiant intensity in that direction of 1683 watt per steradian.

Solar insolation is a measure of solar irradiance over of period of time - typically over the period of a single day.

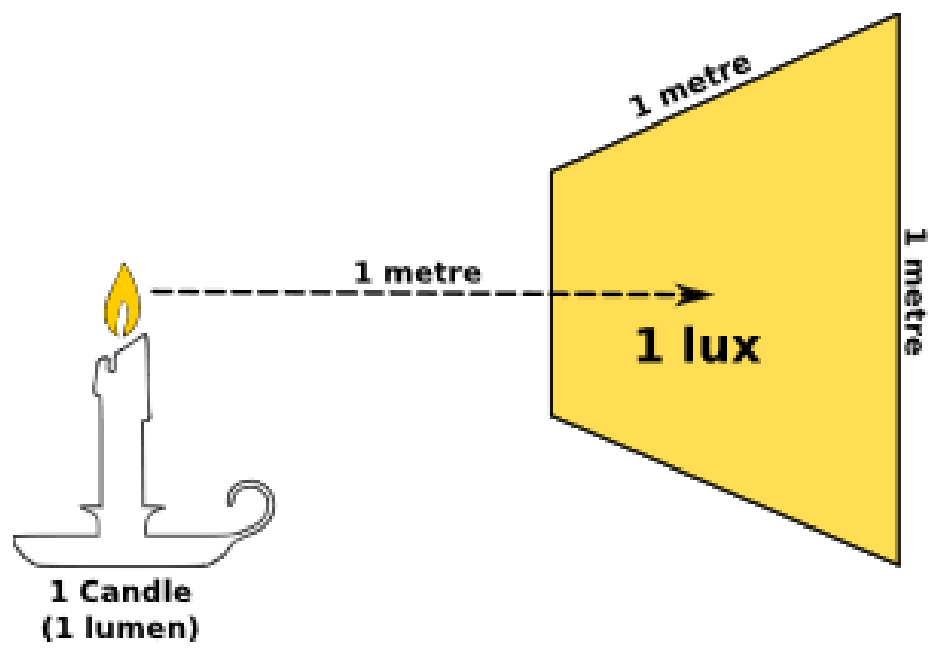

Figure 1.2: The relationship between the illuminance and the candela [3]

A light engineering term exists for the measurement of the rate at which a lamp is able to convert electrical power (Watts) to Light (Lumens), this is referred to as luminous efficacy (LER) (or just efficacy) and is expressed in Lumens per watt.

The relation between $l x$ and the power is given by [3]

$$
1 l x=\operatorname{Power}(W) \times \frac{L E R}{\operatorname{Area}\left(m^{2}\right)}
$$


Figure. 1.2 illustrates the relationship between the illuminance and the candela [3].

\subsection{Channel Noise and SNR}

In OWC systems, the most important challenge is the background noise which is created from ambient light in the environment. Ambient light can rise from sunlight, skylight, advertising signs and fluorescent lamps, or other light sources [14]. The background noise existing in the environment leads to a DC photocurrent that causes a shot noise at the receiver. This noise source is signal independent and is the result of the summation of many independent Poisson distributed random variables. Hence, it can be modeled as a Gaussian distribution [12].

The shot noise variance is given by [15]

$$
\sigma^{2}{ }_{S h o t}=2 q_{e} G^{2} F\left(I_{\text {inc }}\right) B
$$

where $F$ denotes the excess noise, $q_{e}$ denotes the electronic charge constant, $G$ is a PD gain, $B$ denotes the PD bandwidth, and $I_{i n c}$ denotes the photocurrent produced due to the received optical power.

The photocurrent $I_{\text {inc }}$ is given by [16]

$$
I_{\text {inc }}=G A_{r} \int_{\lambda_{1}}^{\lambda_{2}} P_{i n c}(\lambda) \gamma(\lambda) T_{o}(\lambda) d \lambda
$$

where $\lambda_{1}, \lambda_{2}$ are the visible light wavelengths, $\gamma(\lambda)$ is the responsivity of the APD given in A/W at certain $\lambda, A_{r}$ is the effective area of the receiver, and $T_{o}(\lambda)$ is the transmittance of the bandpass optical blue filter.

In addition to the shot noise, there are many different types of noises that are liable for degrading the performance of the VLC system, such as dark noise and thermal noise. Dark 
noise is caused due to the avalanche effect of the APD and is given by [15]

$$
\sigma^{2}{ }_{\text {Dark }}=2 q_{e} G^{2} F I_{d g} B+2 q_{e} I_{d s}
$$

where $I_{d s}$ is a surface dark current, and $I_{d g}$ is a bulk dark current.

Also, due to a random thermal motion of charge carriers, thermal noise is generated. The variance of thermal noise is given by [15]

$$
\sigma_{\text {Thermal }}^{2}=4\left(\frac{K_{B} T_{k}}{R_{L}}\right) F_{n} B
$$

where $K_{B}$ denotes Boltzmanns constant, $T_{k}$ denotes absolute temperature, $R_{L}$ denotes the load resistance, and $F_{n}$ is the photodiode noise figure.

To find the variance of the total noise, we have to apply variance sum law which is an expression for the variance of the sum of two variables. If the variables are independent, the following formula represents the variance of the sum of the variables $\mathrm{X}$ and $\mathrm{Y}\left(\sigma_{X+Y}^{2}=\right.$ $\left.\sigma_{X}^{2}+\sigma_{Y}^{2}\right)$

If $\mathrm{X}$ and $\mathrm{Y}$ are correlated, then the variance sum is $\left(\sigma_{X+Y}^{2}=\sigma_{X}^{2}+\sigma_{Y}^{2}+2 \rho_{x y} \sigma_{X} \sigma_{Y}\right)$, where $\rho_{x y}$ is the correlation coefficient between $\mathrm{X}$ and $\mathrm{Y}[17]$.

Therefore, the variance of the ambient noise $\sigma^{2}{ }_{n}$ is given by

$$
\sigma_{n}^{2}=\sigma_{\text {shot }}^{2}+\sigma_{\text {Dark }}^{2}+\sigma_{\text {Thermal }}^{2}
$$

Table. 1.1 shows the PDF of each type of noise.

In this thesis, Hamamatsu-S8664 APD is considered as a receiver. The simulation parameters and their values are given in Table. $1.2[21]$. 
Table 1.1: PDFs of different types of noise

\begin{tabular}{|c|c|}
\hline \hline Noise & PDF \\
\hline Shot noise & Poisson distribution [18] \\
\hline Dark noise & Poisson distribution [18] \\
\hline Thermal noise & Gaussian distribution [18] \\
\hline ISI noise & Gaussian distribution [19] \\
\hline Clipping noise & Gaussian distribution [20] \\
\hline
\end{tabular}

Table 1.2: Noise parameters and their simulation values

\begin{tabular}{|c|c|c|}
\hline \hline Symbol & Description & Value \\
\hline APD model & $(\mathrm{Si})$ Hamamatsu & $S 8664-1010$ \\
\hline$\lambda$ & Visible light wavelength & $350-750 \mathrm{~nm}$ \\
\hline$G$ & APD gain & 50 \\
\hline$q_{e}$ & Electron charge & $1.9 \times 10^{-19} \mathrm{C}$ \\
\hline$B$ & APD bandwidth & $65 \mathrm{MHz}$ \\
\hline$I_{d s}$ & Surface dark current & $10 \mathrm{nA}$ \\
\hline$I_{d g}$ & Bulk dark current & $5100 \mu \mathrm{A}$ \\
\hline$K_{B}$ & Boltzmanns constant & $1.38 \times 10^{-23} \mathrm{~J} / \mathrm{K}$ \\
\hline$T_{k}$ & Temperature & $298 \mathrm{~K}^{\circ}$ \\
\hline$F_{n}$ & APD noise figure & 0.2 \\
\hline$\gamma$ & Photodetector responsivity & $0.28 \mathrm{~A} / \mathrm{W}$ \\
\hline$R_{L}$ & Load resistance & $50 \Omega$ \\
\hline
\end{tabular}

\subsection{Comparison of Radio Frequency and Visible Light Propagation Channels}

Both radio and visible light waves can be used to enable communications in highly dynamic vehicular environments. Unfortunately, the roles of these two technologies and how they interact with each other in future vehicular communication systems remain unclear. Understanding the propagation characteristics is an essential step in investigating the benefits and shortcomings of each technology.

Comparison of relevant properties such as radiation pattern, path loss modeling, noise and interference, and channel time variation of radio and visible light propagation channels, provides an important insight that the two communication channels can complement each 
other's capabilities in terms of coverage and reliability, thus better satisfying the diverse requirements of future cooperative intelligent transportation systems. The following table summarize the most important observations for applications stemming from propagation channel perspective.

Table 1.3: Comparison of RF and VLC propagation channels for vehicular communications

\begin{tabular}{|c|c|}
\hline \hline Item & \begin{tabular}{c} 
Description \\
\hline Range
\end{tabular} \\
\hline Datarate & $\begin{array}{r}\text { Radio channel is used for longer range and optical } \\
\text { channel for shorter range applications } \\
\text { per-link bit rate and can even sustain a long link } \\
\text { durations }\end{array}$ \\
\hline Complexity & $\begin{array}{c}\text { Optical receiver signal-processing complexity of } \\
\text { vehicular VLC is much lower than its RF counterpart }\end{array}$ \\
\hline Coverage & $\begin{array}{c}\text { VLC communication ensures coverage of only a small } \\
\text { area. Therefore, it is less suitable for use cases which } \\
\text { require omnidirectional transmission }\end{array}$ \\
\hline Cost & $\begin{array}{c}\text { Radio-based solutions have a higher initial deployment } \\
\text { costs. Vehicular VLC has a lower initial cost as LEDs } \\
\text { are common in vehicles today and additional electronics } \\
\text { to enable VLC is inexpensive }\end{array}$ \\
\hline
\end{tabular}

\subsubsection{VLC Advantages and Drawbacks}

VLC is expected to play a big rule in the next generation of wireless communications, due to its unique characteristics and advantages. VLC comes with high bandwidth free of charge which allows for high data rates as shown in Figure. 1.3. VLC also has unlicensed spectrum, and safety for a human body and for high-precision electronic equipment. VLC is also considered more secure than RF and the data transmission is available in addition to the lighting function. Besides these benefits, VLC is a low-cost technology and is easy to implement. Moreover, VLC is a green wireless communication technology [4].

On the other side, VLC has also several drawbacks. Some of the disadvantages are due to the early stage of the VLC technology and could be passed on future as the technology is fully developed. The other ones are due to the usage of the light and its characteristics. It will be 


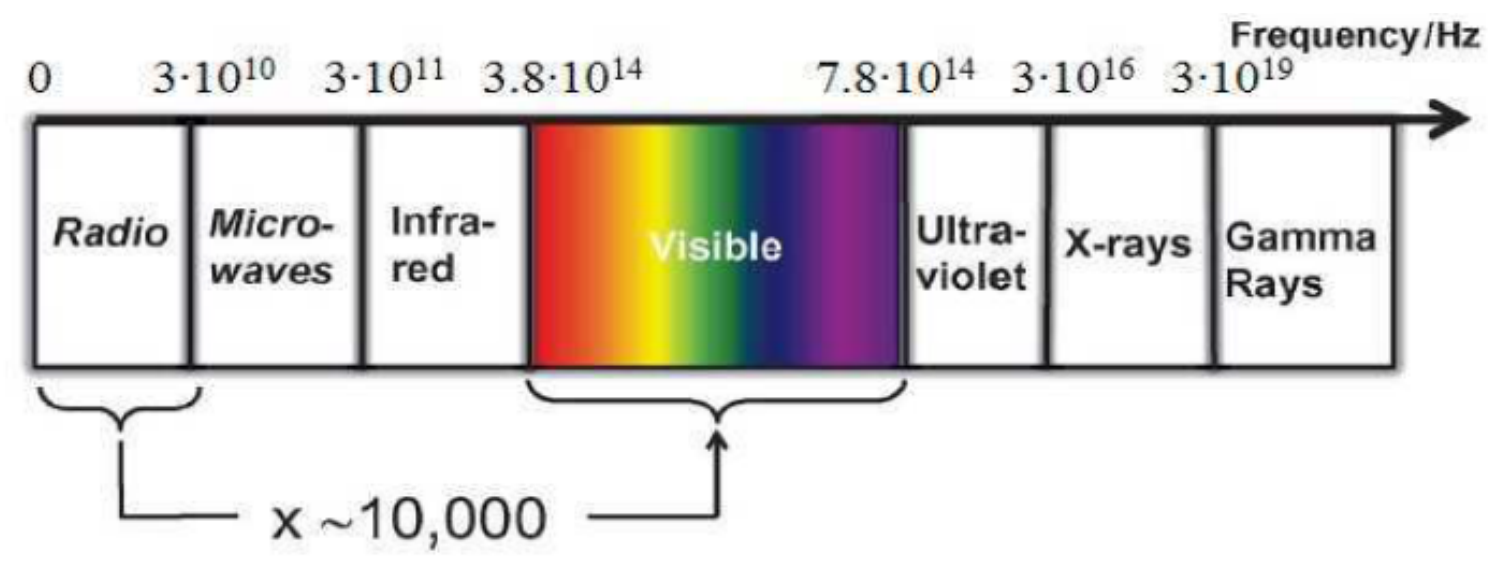

Figure 1.3: Distribution of the electromagnetic spectrum [4].

difficult to completely mitigate the latter issues, but their effects could be reduced or the communication techniques could be adapted to the situations. The main drawbacks of VLC are stringent LOS condition, limited transmission range, and susceptibility to interferences from other light sources.

\subsection{Visible Light Communications in Automotive Applications}

The number of vehicles that use the transportation infrastructure increases every year. Therefore, it is mandatory to keep improving the safety and the efficiency of the transportation system. In spite that vehicles industry has grown much and today's cars are safer than ever before, more than 1.3 million people die every year while 20 to 50 million are injured because of cars accidents [22]. It is estimated that by 2020 road accidents will be the sixth cause of death, with 1.9 million victims yearly [22]. In this context, reducing road accidents and the associated victims is a very serious concern. The United Nations has declared in 2010 a Decade of Action for Road Safety with the purpose of improving the safety of vehicles and roads.

In addition to cars safety systems, there is a strong need for vehicle awareness, obtained 
from different vehicles that work together by sharing information in order to increase the safety, thus, creating a highly-efficient road accident prevention system. Such a system requires high cooperation among vehicles and between vehicles and transportation infrastructure. ITS is the best solution to the road accidents. The ITS consists of intelligent vehicles and intelligent infrastructure, working together to increase the safety and the efficiency of the transportation system [23]. Intelligent transportation system combines advanced wired and wireless communication technologies for data gathering and distribution. By enabling wireless communications among V2V and Vehicle-to-Infrastructure (V2I), the safety and the efficiency of road traffic can be substantially improved. Figure. 1.4 shows the application scenario for vehicular-VLC based.

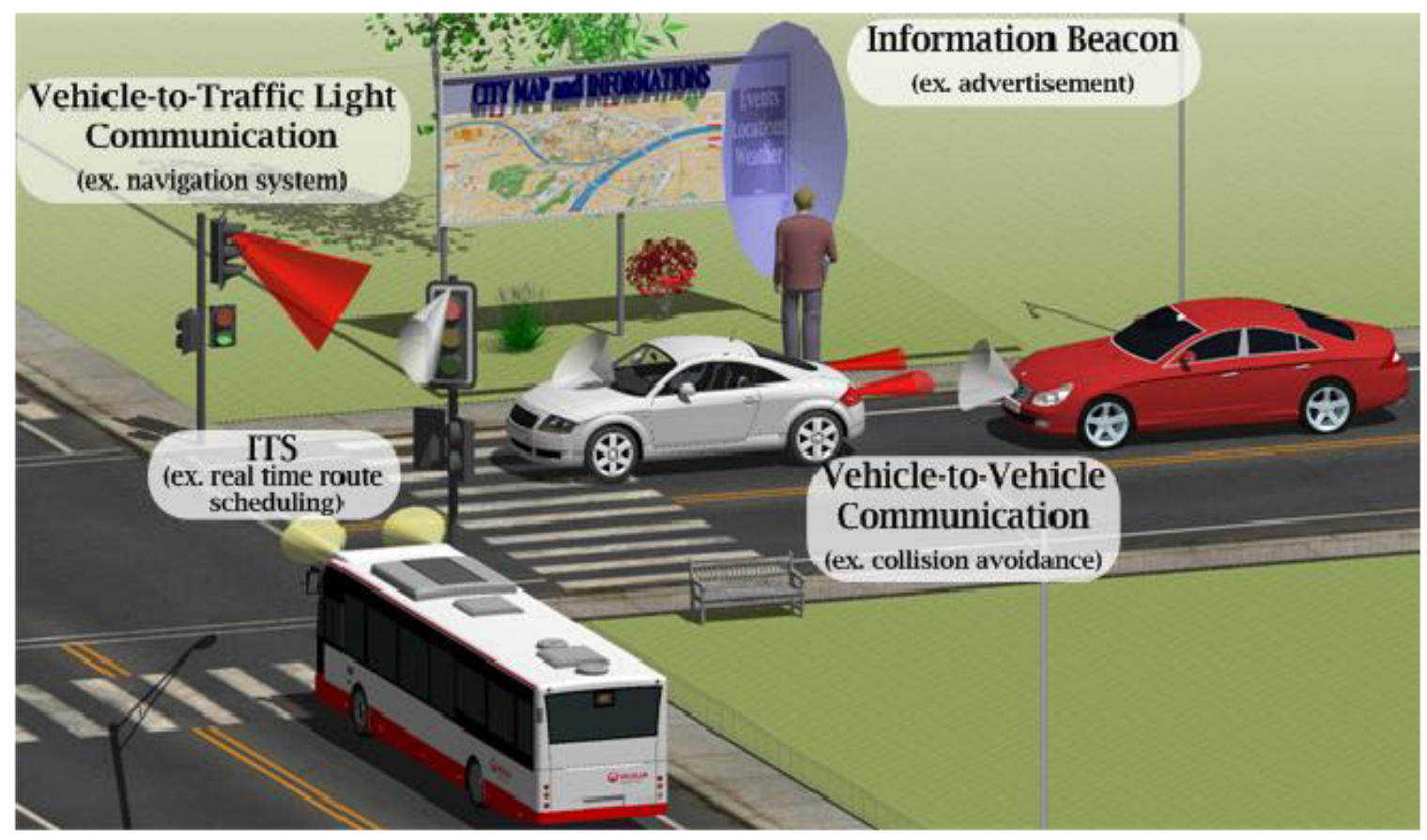

Figure 1.4: Application scenario for vehicular-VLC based [5].

\subsubsection{Usage of VLC in ITS}

Since 1995 the academic and industrial societies are considering Inter-Vehicle Communication (IVC) systems. Recently, VLC was considered as a possible solution to enable IVC. The main 
advantages of VLC usage in automotive applications are represented by the low complexity, reduced implementation cost and the ubiquitous character. All these characteristics can facilitate rapid and wide market penetration, which represents a strong considerate in favor of the VLC.

Due to the LEDs features, car manufacturers think of replacing the classical halogen lamps with LED lighting systems. LEDs are highly reliable, energy efficient and have a lifetime that exceeds by far the classical light sources. Nowadays, and as illustrated in Figure. 1.5, vehicles lighting systems based on LEDs are common.

Moreover, LEDs based traffic lights becoming more popular and is beginning to be used on an extended scale. Normally a large number (100-200) of LEDs are used for the traffic light that offer the signaling function and the possibility for data transmission. Also, most of the street lighting will be LEDs based in the near future, so the road illumination will also be able to provide communication support for the case of Infrastructure-to-Vehicle (I2V)-VLC.

From the above, one can see that LED-based lighting will be part of the transportation system, being integrated with vehicles and in the infrastructure. The large geographical area in which LEDs lighting will be used, combined with VLC technology will allow ITS to gather data from a widespread area and can enable the distribution of high-quality communications. These additional functions will be possible without affecting the primary goal which is signaling or lighting in any way. This leads to a fact that VLC is able to satisfy the requirements imposed in vehicular networks in real working conditions as confirmed.

\subsubsection{VLC in the ITS / State of the Art}

Many research groups have investigated the usage of VLC in automotive communication to increase traffic safety by allowing $\mathrm{V} 2 \mathrm{~V}$ and $\mathrm{I} 2 \mathrm{~V}$ communications. The simulation and experimental results obtained so far, prove that the use of VLC for road safety applications is possible, but to enable widespread implementation, the performances of such systems still need more work and more improvement [4]. 

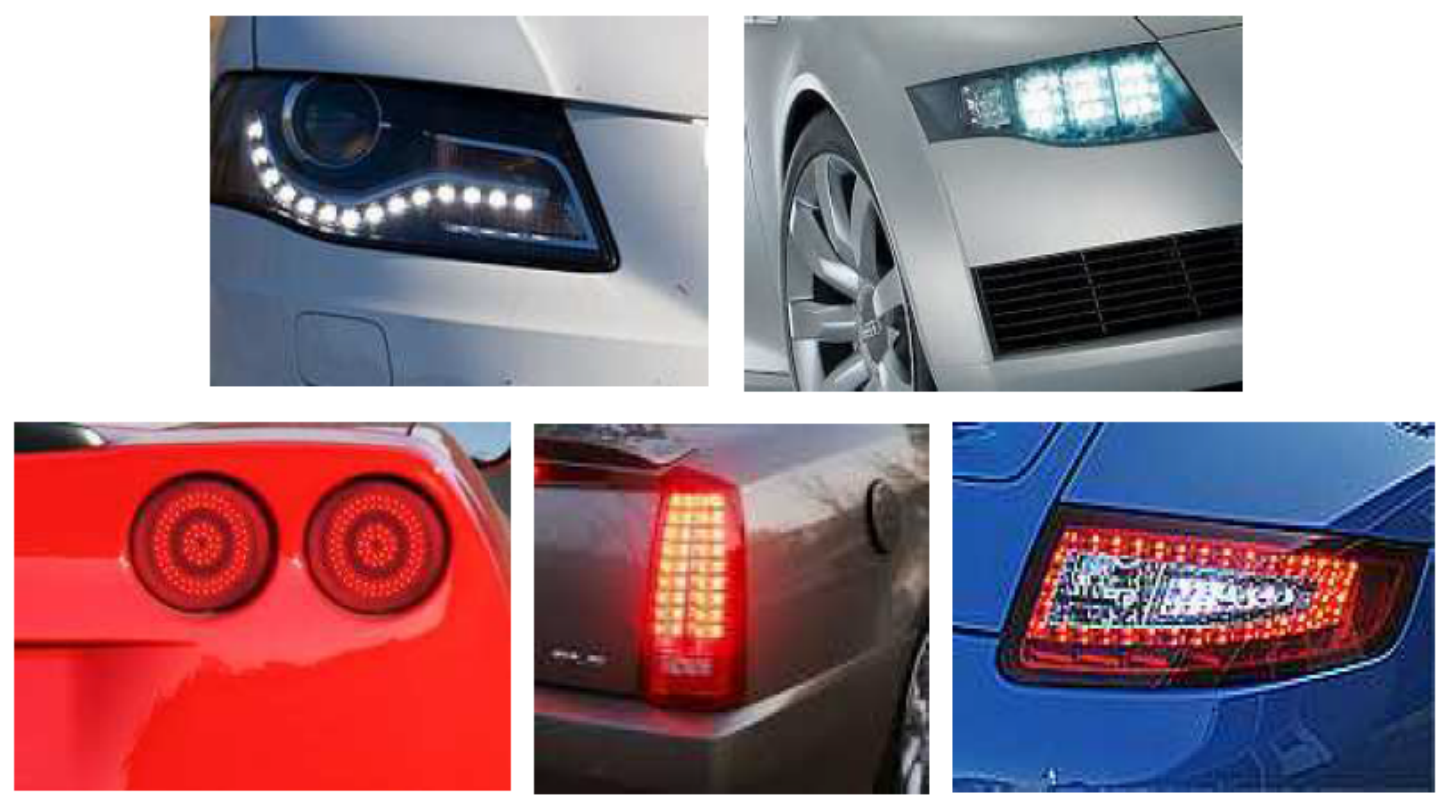

Figure 1.5: Integration of LEDs lighting systems in series vehicles [6].

In the following paragraphs, we present a brief review of the work of some of the most representative research groups in the area of VLC automotive usage.

The group of Smart Lighting Engineering Research Center of Boston University (USA) is involved in the usage of VLC for IVC. The research team has developed a prototype used for vehicular networking based on optical transceivers. The system uses short-range directional optical transceivers to share vehicle state data. Also, they performed a comparative analysis between omnidirectional 802.11 RF communications and directional VLC, with application in vehicular communications is presented in [24]. The results show that in high traffic density, VLC offers better performances in terms of packet delivery ratio, throughput, and average packet delay, at the cost of a shorter communication range [25].

The group of professor Knightly from Rice University (USA) is another research group in vehicular communication VLC based. They presented a detailed analysis of Vehicular VLC based networks. They developed a research platform with high robustness to noise on which they made experiments in different conditions and for different scenarios. The experimental results show that VLC offers the possibility of robust vehicular communication in real traffic conditions. Experiments showed that V2V-VLC system is resilient to sunlight noise except 
for the case of a direct line of sight with the sun. Also, their results show that in dense traffic conditions, VLC satisfies the latency and the reachability requirements imposed by the vehicle safety applications [26].

G. Pang from the university of Hong Kong (China) and his research group are one of the pioneers of VLC systems for traffic safety. He considered that LEDs have the potential to replace the classical light sources and to be integrated with traffic lights, traffic signaling devices or into traffic display boards dut to the LEDs fast switching ability. This group proposed the replacement of all traffic lights and signaling devices with LEDs to reduce power consumption and to increase traffic safety. Also, they confirmed that the LEDs long life expectancy, even in unfriendly working conditions, is extremely important since a burned traffic light can be a major risk factor. On the other hand, the communication capabilities of the LEDs can be used to further increase the safety of the transportation. Under these circumstances, the authors demonstrated the dual use of LEDs in the ITS: signaling and communication. To support their arguments, the research group presented a prototype traffic light that besides the traditional signaling purpose also transmits audio information through visible light [27]. Moreover, the research group proposed an intelligent traffic light system for the broadcasting of vehicle location and navigation information with audio support for the driver. The same group presented a different approach for traffic light to vehicle communication in [28].

The researchers from the Nagoya University (Japan) are one of the early groups working in the ITS area. They have an inspiring background in the development of ITS-VLC base. In 2005 they proposed a parallel VLC system meant to broadcast traffic safety information from a LED-based traffic light to a high-speed camera-based receiver [29]. In 2007, they proposed a novel concept of VLC receiver prototype that aims to solve the main problems associated with the use of VLC in the ITS such as the necessity of long-distance high-speed transmission under the dynamic conditions [30]. The experimental results showed a $2 \mathrm{Mb} / \mathrm{s}$ communication speed and a Bit Error Rate (BER) was below $10^{-6}$ for distances up to 40 meters. The performances of the high-speed camera based receivers were improved in the 
years that followed with the development of the coding scheme and as higher performance cameras became available. The communication distance was increased up to distances of 120 meters, however at a high BER of $10^{-2}$ [31].

The collaboration between Shizuoka University (Japan) and the Applied Optics laboratory from Toyota enabled the development of a high-performance VLC system. They have developed a high sensitivity Complementary Metal-Oxide Semiconductor (CMOS) image sensor which is able to achieve 1000 (frame/second). With the integration of the image sensor in a VLC receiver, their $\mathrm{V} 2 \mathrm{~V}$ prototype was able to achieve data rates of $10 \mathrm{Mb} / \mathrm{s}$ for distances that can go up to 20 meters [32]. The communication range of the systems can be increased up to 50 meters by decreasing the data rate to $32 \mathrm{~kb} / \mathrm{s}$, or even up to 100 meters for data rates of $2 \mathrm{~kb} / \mathrm{s}$ [33].

One of the leading groups in the research of VLC usage for automotive applications is in Portugal, at the university of Aveiro. The group has proposed and analyzed in detail the use of LED-based traffic lights as Road Side Units (RSU) as apart of the ITS for I2V data broadcast. The research group has considered the usage of PDs based receivers and proposed the use of DSSS inverse keying as a modulation technique [10]. The experimental results showed that this modulation technique is suitable for outdoor VLC since it reduces the effect of noise produced by artificial light sources [34]. The proposed system has been tested for outdoor conditions both in the daytime and at the night time. The system is able to transmit data up to more than 40 meters with a BER between $10^{-6}$ at 10 meters and $10^{-2}$ at 45 meters with the data rate $20 \mathrm{~kb} / \mathrm{s}$.

\subsection{VLC Research Direction and Future Challenges}

Even though, the discussed methods enhance the communication data rates, still very high data rates (more than a Gbit/s) are unachievable even with optical filtering and equalization techniques. Also, providing a high-speed uplink is an additional challenge for VLC [35]. Another challenge for VLC is to cooperate with another wireless standard such as RF. The 
work in [36] examined the combination of a high-speed down link and a lower speed RF wireless LAN and showed that the combined system possess some benefits in terms of latency and throughput. Also, the work in OMEGA project aims to combine different RF and optical wireless to achieve the desired performance [37].

In [38], the IEEE 802.15.7 group showed that the integration of lighting and communication provides significant potential for VLC technology. They reported two main challenges in communication in this scenario, flicker mitigation and support for dimming. Moreover, they presented few techniques to mitigate flicker and support dimming as defined in the IEEE 802.15.7 VLC standard. Besides, they found that there are many technical challenges must be addressed to realize the full potential of VLC technology. The first challenge is, the channel models for VLC are not well understood, especially for NLOS for outdoor environments, and there is an active area of research for channel models and platforms for VLC. Also, the networking of the light sources and upgrading current infrastructures to support communication is another challenge, which requires support from the lighting industry. They conclude their work as, with continued growth in LED-based light sources and the need for multi-Gb/s data distribution, VLC, being developed as a global industry standard in IEEE 802.15.7, promises to be a very attractive candidate as a future high data rate and power-efficient technology

Furthermore, using the VLC in outdoor application still facing many problems since the channel is highly affected by the metrological conditions, besides the noise which is coming from the sun and other light sources. Finally, the VLC system cannot be used as a long distance communication system.

\subsection{Motivation of the Work}

The area of ITS is directly related to human and material safety. It uses many technologies to prevent losses and reduce casualties. Vehicular communication based on VLC is an important concept for road safety applications in ITS. VLC systems, though with high potential to be 
an integral part of ITS, because of ubiquitous infrastructures support have not been deeply investigated. Therefore, there is a great need to explore this research area and analyze its novel applications in road safety. Furthermore, VLC systems are cost effective, use LEDs and the unregulated visible spectrum. Hence, they are expected to offer broad potential applications. The research work in this thesis is focused on the V2V-VLC system.

\subsection{Contribution}

This thesis work investigates aspects in the realization of V2V-VLC systems. This work was developed aiming for the road safety application area. There are many original contributions in this research work. The major contributions are

- Investigating a conceptual $(2 \times 2)$ MIMO-V2V-VLC system and its associated modules. We characterize an outdoor optical channel which is a key point in the V2V-VLC system.

- Using an appropriate modulation scheme for communication systems is another key requirement. We have proposed and developed an adaptive modulation scheme based on O-OFDM modulation technique for this application. Also, we present a pre coding scheme for the V2V-VLC system.

- We investigate different precoding and equalization schemes for $2 \times 2 \mathrm{MIMO}$ for the V2V-VLC system considering flickering/dimming control and the nature of outdoor VLC channel as a frequency selective channel.

- Due to the nature of the visible light, shadowing is a significant issue especially over short distances and narrow Filed of View (FOV). This can seriously impair communications in fast varying outdoor V2V-VLC environments. We proposed a method to take advantage of the optical diffraction phenomenon to overcome shadowing by employing a receiver with a wide FOV. We have modeled the shadowing effect for visible light by a Bimodal 
Gaussian distribution and derived the probability of error for no shadowing, moderate shadowing, and severe shadowing conditions.

- Since the noise is a serious issue in VLC- outdoor application, two novel denoising schemes are proposed. The first one is by using differential receiver and the second scheme is by using MLE algorithms.

- Apart from the above, we have studied a scenario in which an outdoor VLC channel with Foglets assisted road asset reporting system, where Foglet is considered an important part of ITS.

The work in this thesis was reported in many reputable conferences, journals, and as a book chapter.

\subsection{Thesis Outline}

The work reported in this thesis is divided into eight chapters as shown in Figure. 1.6. The contents of the following chapters are briefly described.

- Chapter 2 presents the proposing a $(2 \times 2)$ MIMO channel for V2V-VLC by considering LOS and NLOS components. We analyze the effects of infinite number of reflections. Then, we combine it analytically into a mathematical formulation using standard numerical methods to get the CIR. Afterward, we utilize the obtained CIR to derive and investigate some statistical properties such as BER, received power, mean access delay, Root Mean Squared (RMS) delay spread, coherence bandwidth and coherence time in both directions of vehicles movement.

- In chapter 3, we use O-OFDM with an adaptive modulation scheme to mitigate ISI, and also to improve data rate. We consider Direct Current Optical Orthogonal Frequency Division Multiplexing (DCO-OFDM) and Asymmetrically Clipped Optical Orthogonal Frequency Division Multiplexing (ACO-OFDM) schemes for VLC channel. 


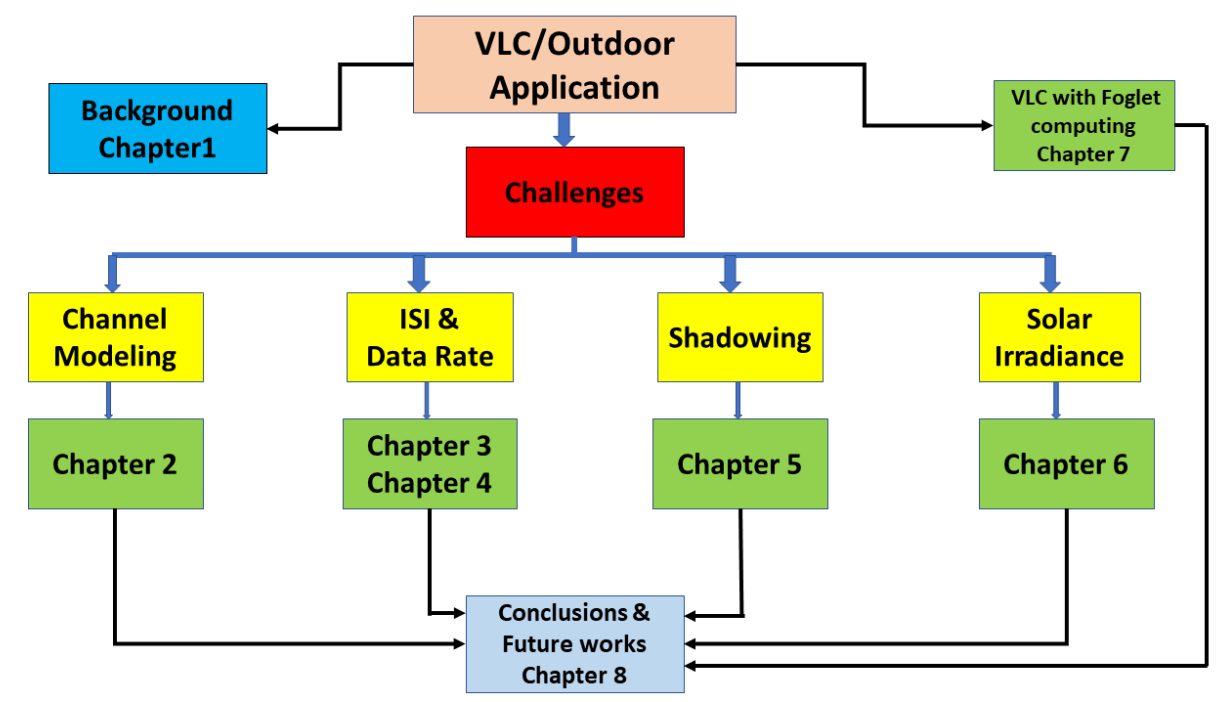

Figure 1.6: Summary of the thesis outline.

We analyze the proposed algorithm with numerical simulation and prove its superiority by performing a comparative analysis with conventional modulation schemes. Data rates up to 50 Mbps with reduced BER is achieved with the proposed algorithm.

- In chapter 4 , we investigate precoding and equalizing scheme for the V2V-VLC system considering flickering/dimming control and the nature of the outdoor VLC channel as a frequency selective channel to enhance the system's performance. We present the precoding matrix, as well as the equalization matrix for Zero Forcing (ZF), Maximum Likelihood (ML), and Minimum Mean-Square Error (MMSE) schemes. We compare the system performance of the three schemes, where the MMSE scheme shows a noticeable improvement.

- In chapter 5, we study the shadowing effect on the V2V-VLC system. We propose a method to take advantage of the optical diffraction phenomenon to overcome shadowing by employing a receiver with a wide FOV. We have modeled the shadowing effect for visible light by a bimodal distribution and derived the probability of error for different types of shadowing.

- The solar irradiance effect on the V2V-VLC system and different denoising schemes are 
presented in chapter 6. In this chapter, we explore the performance of the V2V-VLC systems during the daylight (under sunlight effect). The performance of the system is investigated in terms of SNR, BER, and data rate under three different scenarios. Firstly, the solar irradiance is collected without any optical filtering in front of the receiver. Secondly, the solar irradiance is collected with an optical filtering in front of the receiver. Thirdly, the solar irradiance is collected under our proposed method for filtering the received signal by the differential receiver. We compare the results of the three scenarios where the differential receiver method shows noticeable improvement in the system performance. Also, we propose denoising scheme by MLE technology. We discuss and propose efficient method to overcome the effect of the solar irradiance in VLC link used for V2V-VLC system. We propose kNN algorithm-MLE based adaptive filter to combat the effect of solar irradiance. Moreover, we use supervised algorithm in the analysis. Both denoising schemes are discussed in details.

- We use the VLC link between Foglets and smart vehicles and discuss its safety and suitability for the road assets infrastructure as compared with conventional RF links in chapter 7. We model this VLC link and discuss its luminosity and BER characteristics.

- Finally, chapter 8 reports the concluding part of the thesis. Some guidelines for future study and investigation are also discussed.

\subsection{Chapter Summary}

This chapter introduces the concept, the architecture design, the advantages and the drawbacks of VLC system. Also, different types of noise and SNR are introduced. The potential usage and the role of the VLC in the ITS have been discussed. In ITS, VLC appears to be the solution, especially for urban high-traffic densities. This chapter, also, highlights the current trends in the development of VLC systems and the challenges in the domain. 


\section{Chapter 2}

\section{Multipath Channel Model for V2V-VLC System}

Accurate VLC channel models are necessary for better system design. Using VLC in outdoor applications has few challenges such as severe weather conditions, sunlight, and ambient light. Weather conditions such as heavy fog or snow could decrease the communication range. These degrading effects can be minimized by using highly sensitive receivers. Another strong concern is direct sunlight or strong ambient light, which could saturate the receiver [39].

In literature, most of the optical wireless works in the VLC environment are focusing on indoor channel modeling, while there is little work on outdoor applications, specially multipath mobile V2V channel.

In this chapter, we investigate a novel V2V-VLC system in a rich scattering environment considering both LOS and NLOS paths. We employe a pair of headlights as transmitters in one vehicle, and a pair of taillights as receivers in the adjacent vehicle, making the system analogous to a $2 \times 2$ MIMO communication link. A closed-form expression of the CIR is derived, and various channel parameters are obtained. Further, the results of the V2V-VLC model are compared with other models found in the literature. The results show a high degree of accuracy in BER and received power. Also, the results show from contributions of the emitted light, double-bounce reflections and up can be neglected for outdoor applications. 
Moreover, it is shown that the V2V channel RMS delay spread is similar to a Rician distribution. Finally, the V2V-VLC channel coherence time is found to be at least an order of magnitude larger than that of RF channels, indicating that the V2V-VLC channels are much stable.

Our contributions in this chapter are as follows:

1. Developing a $2 \times 2$ MIMO channel for V2V-VLC by considering LOS and NLOS components from the reflectors in a typical street environment based on upcoming IEEE 802.15.7r1.

2. Combining the effects of infinite number of reflections analytically into a mathematical formulation using standard numerical methods.

3. Utilizing the analytical model to derive and investigate some statistical properties such as BER, received power, mean access delay, RMS delay spread, coherence bandwidth, and coherence time in both directions of vehicles movement.

\subsection{Related Work in Channel Modeling for VLC}

In this section, some of the efforts done in the VLC in vehicular applications will be reviewed.

A mathematical model for the V2V-VLC system was presented in [7]. They used a market-weighted headlamp beam pattern considering both the LOS and the NLOS links. They considered the impact of NLOS path coming from the road surface reflection only. Also, they considered a good weather condition in their analysis. In [40], the authors extended their work in [7], but they assumed a wet surface road and the reflection is not Lambertian. They investigated the system's performance in terms of BER and the hight of the receiver.

The authors in [41] proposed a measurement-based time variant non-clear sky channel model for the I2V-VLC system. Also, they consider the dynamic characteristics of background radiation to enable more realistic and accurate prediction of the VLC system performance in an outdoor application. Moreover, they introduced a new receiver design with dual-reception and 
effective ambient-light rejection capabilities which employs the selection diversity technique in order to mitigate the impact of ambient-light noise due to daylight.

In [42], a time function of the V2V channel path loss caused by vehicle mobility had been obtained using the video data collected from cameras mounted on driven vehicles. A computer vision techniques were used to identify the location of the taillights of the transmitting vehicle with respect to the receiving vehicle.

The authors in [43] used a ray-tracing scheme employing commercial light tools software to evaluate channel delay profiles obtained from CIR for multiple LOS links and a less number of NLOS delay taps for crossroad and metropolitan street under the V2V and V2I communication links. They concluded that the delay profile from the V2I link and metropolitan scenario had more dispersive channel characteristics due to the reflection and the diffusion of the visible light.

In [44], a channel model of OWC systems during rainfall was proposed. The authors considered outdoor optical wireless systems where optical transmitter communicates directly along point-to-point LOS propagation links. They considered the scattering of optical light through raindrops in the propagation channel and derived the PDF of the received signal power.

The authors in [45] evaluated the VLC channel model in the crossroad and metropolitan scenarios based on the practical VLC light sources. They used light tools and CATIA V5 tools to simulate their work.

In [46], the authors used an image sensor instead of PDs as a receiver, where this technique considers as an attractive solution for the outdoor mobile applications. They introduced the pinhole camera model concept to express a motion of the VLC transmitters. Moreover, they used the motion models to simulate and analyze VLC channel fluctuation. 


\section{$2.2(2 \times 2)$ MIMO V2V-VLC System Model}

This section discusses the system model in detail. The V2V-VLC system which is shown in Figure. 2.1 is comprised of two vehicles communicating with each other by utilizing headlights and taillights. Headlights of the first vehicle act as a transmitter and taillights of the second vehicle act as a receiver. Since each vehicle has two headlights and two taillights, a $2 \times 2$ MIMO link can be considered. The LOS and NLOS paths are considered in this work.

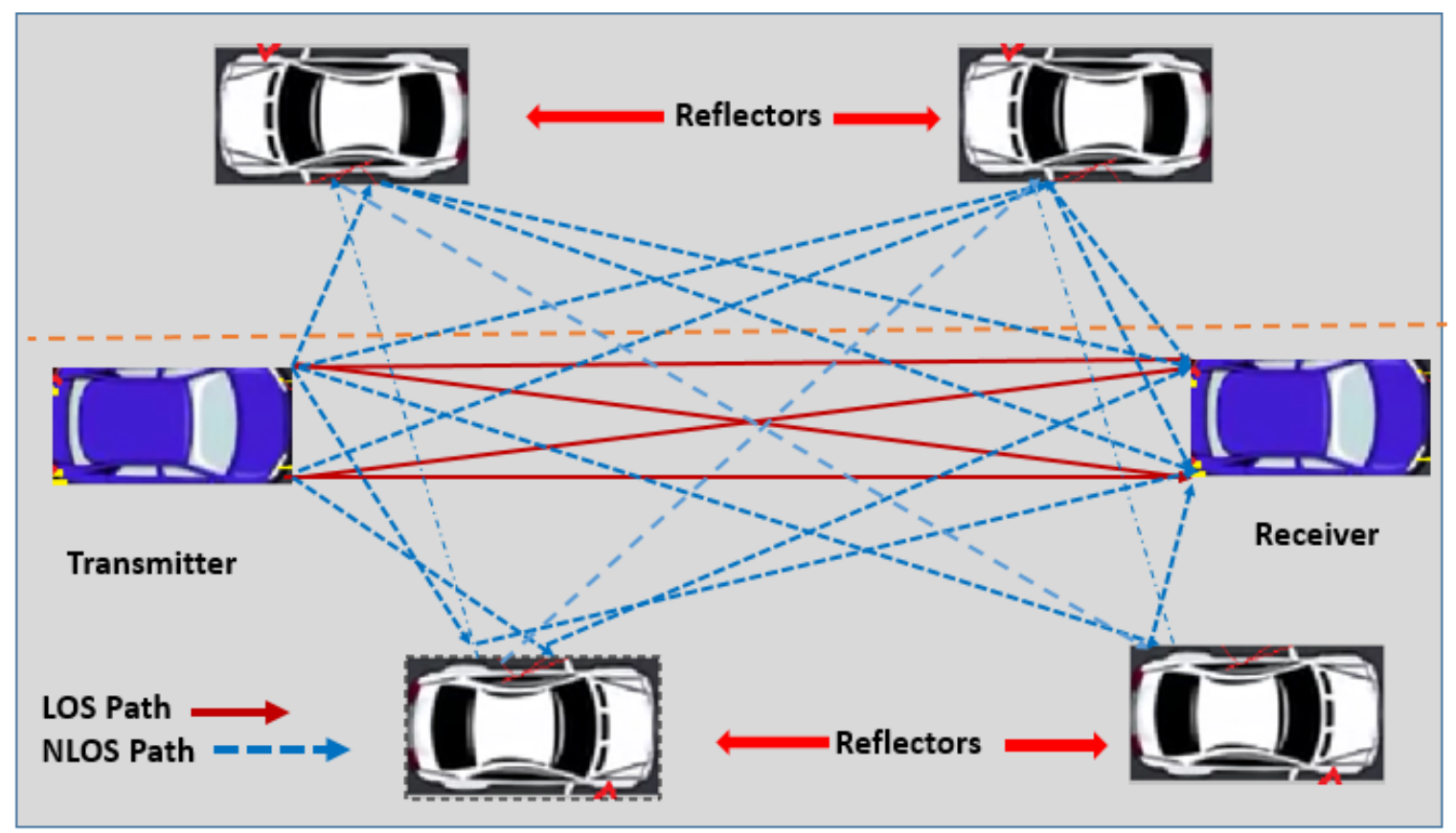

Figure 2.1: $(2 \times 2)$ MIMO model for V2V-VLC showing the LOS and NLOS paths.

\subsubsection{The V2V-VLC System Considerations}

LEDs at the transmitter side and APDs at the receiver side are considered. The market-weighted head-lighting pattern source for the transmitter and receiver [47] is used in the simulation. These types of lights provide good road illumination, as well as very good anti-glare properties [48]. Moreover, practical outdoor VLC links are considered in this work including parked and passing vehicles acting as reflectors. 
Each reflector acts as a receiver when the signal is received from the transmitting vehicle and subsequently as a transmitter when the signal is reflected to the receiving vehicle. Because of the reflectance characteristics depend on the reflector's nature and physical state, and it also changes with weather conditions, a Bidirectional Reflectance Distribution Function (BRDF) is used to model the reflectors in this work. A BRDF model can effectively model the polarimetric signatures of the object surface such as paints or metal [49]. The BRDF is given by [49]

$$
f\left(\theta_{i n c}, \phi_{i n c}, \theta_{r e f}, \phi_{r e f}, \lambda\right)=\frac{d L_{r}\left(\theta_{r e f}, \phi_{r e f}\right)}{d E_{i}\left(\theta_{i n c}, \phi_{i n c}\right)}
$$

where $\theta_{i n c}, \phi_{i n c}$ respectively denote the incident polar and azimuth angles, $\theta_{r e f}, \phi_{r e f}$ respectively denote the reflected polar and azimuth angles, $\lambda$ is the wavelength, $L_{r}$ is the irradiance scattering from the surface, and $E_{i}$ is the irradiance incident onto the surface.

As outdoor VLC channels undergo fast fading effects, a frequency selective channel model is considered in this work for modeling the system [39].

Referring to the Ministry of Transportation of Canada regulations [50], and by using the data provided in [47], and the headlight radiation pattern shown in Figure. 2.2, we observe the following

1. For the effective luminous intensity of 1000 candela (cd), the road width should be about $10 \mathrm{~m}$ and the longitudinal distance should be ranging from 80 to $100 \mathrm{~m}$.

2. The safe distance between vehicles traveling at a speed of $80 \mathrm{~km} / \mathrm{h}$, and moving in the same lane must be at least $23 \mathrm{~m}$.

3. The safe distance between vehicles in parallel lanes must be at least $3 \mathrm{~m}$.

Due to the above regulations, the V2V-VLC model can have up to 6 reflecting vehicles within the testing area and only one LOS path. 
(a) Isocandela diagram (cd)

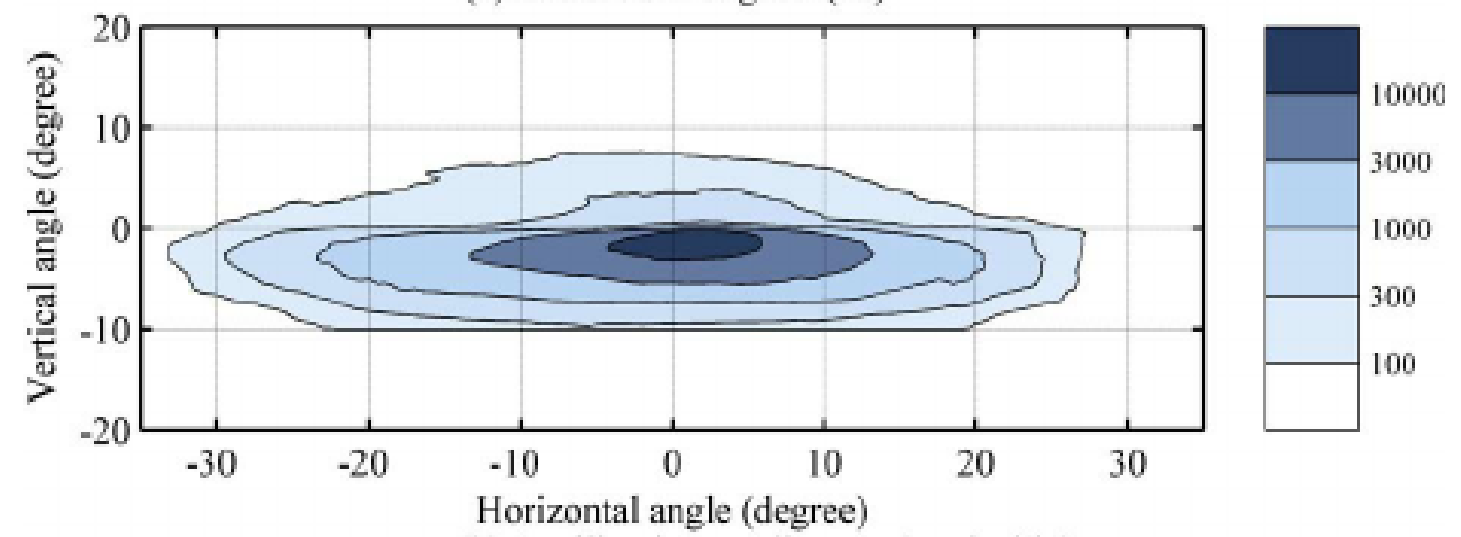

(b) Iso-illuminance diagram (vertical $\mathrm{lx}$ )

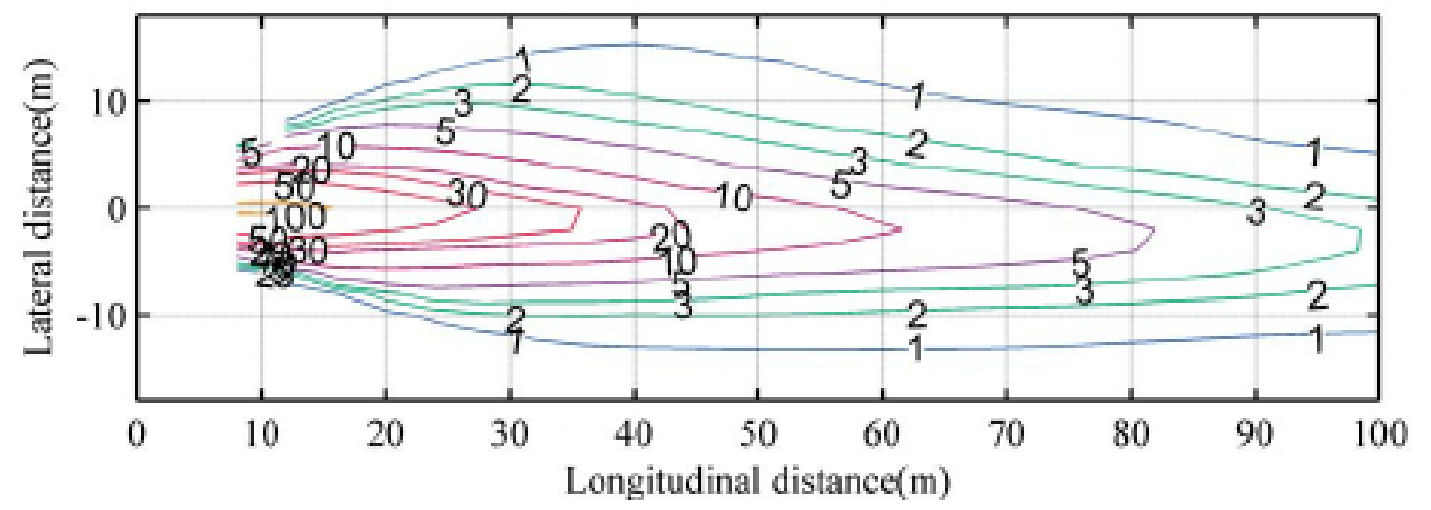

Figure 2.2: Headlight optical radiation pattern [7]

\subsubsection{System Model}

The block diagram of the V2V-VLC system is shown in Figure. 2.3. The transmitted serial data stream of the $2 \times 2$ MIMO model is converted into two parallel streams using a Serial-to-Parallel (S/P) converter. Each data stream is intensity modulated and transmitted through LED headlights. At the receiver taillights, PDs convert the light into electrical signals using direct detection. The received signal can be written as

$$
y_{r}(t)=\gamma x(t) \otimes h(t)+n(t)
$$




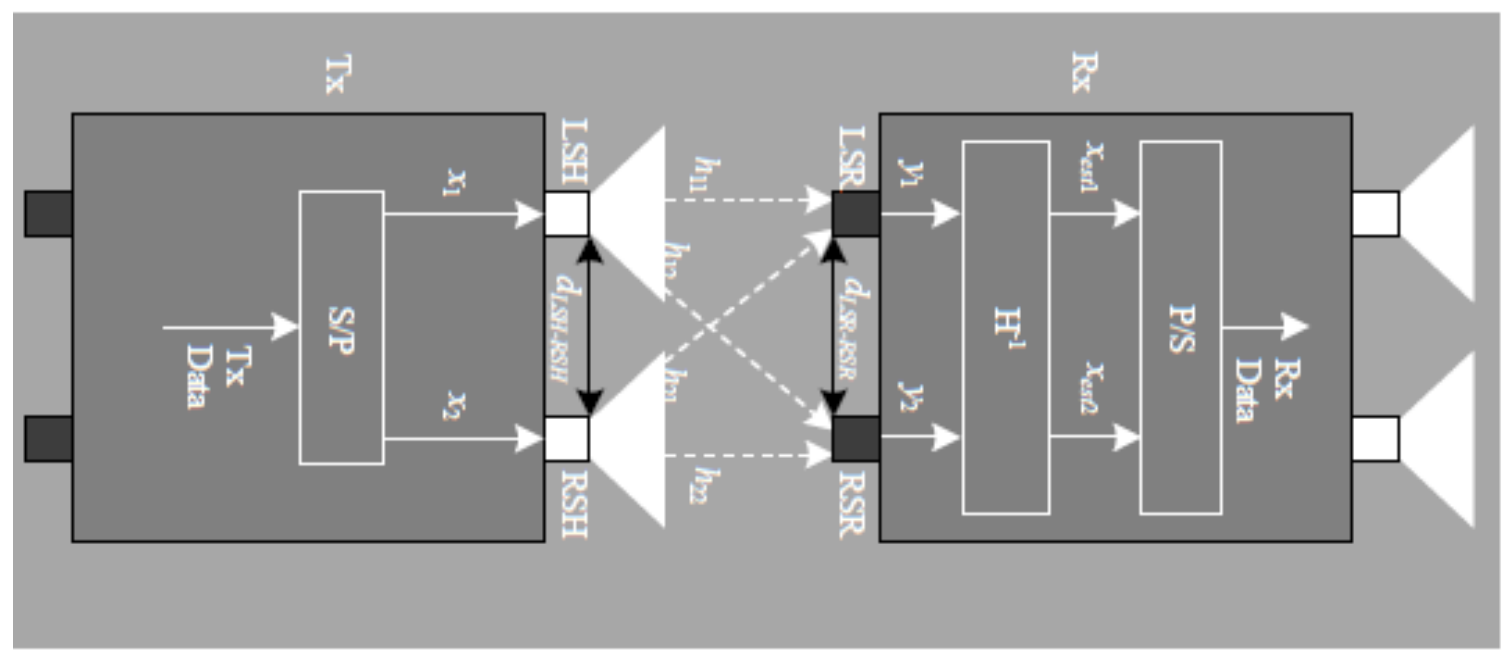

Figure 2.3: Block diagram of a $2 \times 2$ MIMO link of V2V-VLC system

where $x(t)$ is the transmitted signal, $y_{r}(t)$ is the received signal, $h(t)$ is the CIR, $\gamma$ is the PD responsivity, $\otimes$ is the convolution sign, and $n(t)$ is the noise.

In vector notation (2.2) is given by

$$
\mathbf{y}_{\mathbf{r}}=\gamma \mathbf{H x}+\mathbf{n}
$$

where $\mathbf{x}$ and $\mathbf{y}_{\mathbf{r}}$ are transmitted and received vectors, respectively, $\mathbf{H}$ is the channel matrix which consists of the LOS and the NLOS components, and $\mathbf{n}$ is the noise vector.

For a $2 \times 2$ MIMO system, (2.3) can be rewritten as

$$
\begin{aligned}
& \mathbf{y}_{\mathbf{r}}=\left[\begin{array}{ll}
y_{1} & y_{2}
\end{array}\right]^{\mathbf{T}} \\
& \mathbf{x}=\left[\begin{array}{ll}
x_{1} & x_{2}
\end{array}\right]^{\mathbf{T}} \\
& \mathbf{n}=\left[\begin{array}{ll}
n_{1} & n_{2}
\end{array}\right]^{\mathbf{T}}
\end{aligned}
$$


The channel matrix is given by

$$
\mathbf{H}=\left[\begin{array}{ll}
h_{11} & h_{12} \\
h_{21} & h_{22}
\end{array}\right]
$$

where $x_{1}$ and $x_{2}$ are the transmitted signals from first and second transmitters $s_{1}$ and $s_{2}$, respectively, $y_{1}$ and $y_{2}$ are the received signals by both receivers $r_{1}$ and $r_{2}$, while $n_{1}$, and $n_{2}$ represent the Gaussian noise at receiver $r_{1}$, and $r_{2}$, respectively.

\subsection{Channel Impulse Response and Transfer Function}

The total CIR of the V2V-VLC model, $h(t)$ is given by

$$
h(t)=h(t)_{L O S}+h(t)_{N L O S}
$$

By performing Fast Fourier Transform (FFT) on (2.8) we get

$$
H(\omega)=H(\omega)_{L O S}+H(\omega)_{N L O S}
$$

\subsubsection{Impulse Response and Transfer Function of the LOS Component}

The $2 \times 2$ impulse response matrix $\mathbf{H}_{L O S}$ between the transmitters and the receivers for the LOS path can be written as

$$
\mathbf{H}_{L O S}=\left[\begin{array}{ll}
h_{11} & h_{12} \\
h_{21} & h_{22}
\end{array}\right]
$$

Any element in $\mathbf{H}_{L O S}$ is expressed as

$$
h_{r s}(t)=G_{r s} \delta\left(t-\tau_{r s}\right) \quad r=1,2, \text { and } s=1,2 .
$$


where $G_{r s}$ denotes the gain factor accounting for the losses and gains between the source $s$ and the receiver $r, \tau_{r s}=R_{r s} / c$, denotes the time delay, $R_{r s}$ denotes the distance between the source $s$ and receiver $r, c$ is the speed of light, and $\delta()$ denotes the impulse function.

The gain factor, $G_{r s}$, is given by [51]

$$
G_{r s}=\frac{\cos \left(\phi_{r s}\right) \cos \left(\theta_{r s}\right) A_{r} T_{s}\left(\theta_{r s}\right) g\left(\theta_{r s}\right)}{\pi R_{r s}^{2}}
$$

where $\phi_{r s}$ denotes the emitting angle between source $s$ and receiver $r, \theta_{r s}$ denotes the incident angle between source $s$ and receiver $r, A_{r}$ denotes the effective area of the receiver, $T_{s}\left(\theta_{r s}\right)$ denotes the signal transmission coefficient of an optical filter, and $g\left(\theta_{r s}\right)$ denotes the concentrator gain. Figure. 2.4 shows the relevant angles for the transmitter, the receiver, and the reflector.

In the frequency domain, the LOS transfer function of (2.11) is given by

$$
H_{r s}(\omega)=G_{r s} e^{-j \omega \tau_{r s}}
$$

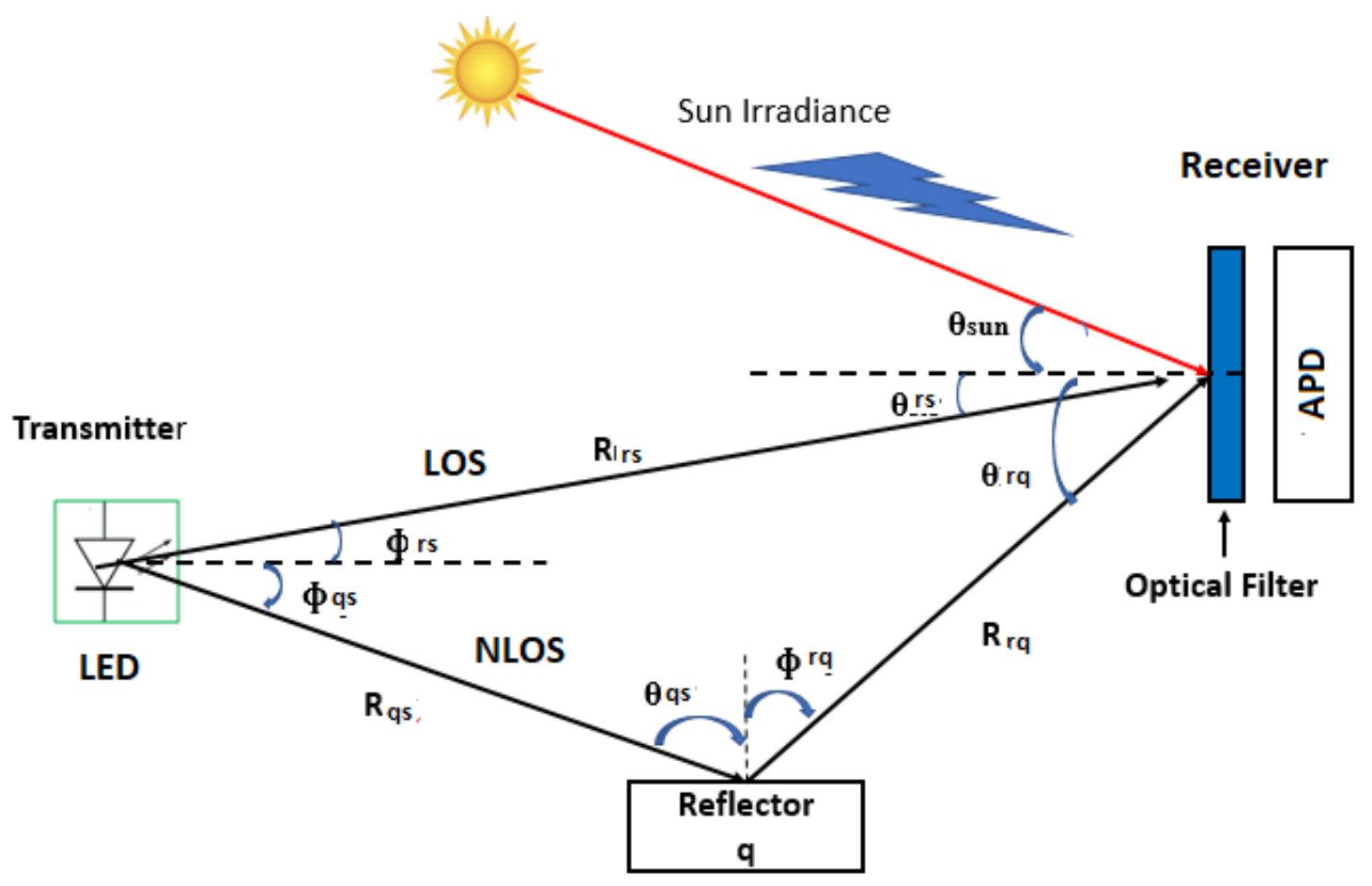

Figure 2.4: Emitting and incident angles and other related parameters. 


\subsubsection{Impulse Response and Transfer Function of the NLOS Component}

In this work, we assume each reflector surface can be divided into $N$ small surfaces (subsurfaces) elements numbered from $(1,2, \ldots, N)$ with equal areas $A_{i}$ and equal reflectivity factors $\rho_{i}<1$ ( $i=$ $1,2, \ldots, N)$ as shown in Figure. 2.5.

When the transmitted light incidents on the reflector surface, the surface element will be considered as a receiver. Thus, the impulse response matrix $\mathbf{A}$ between the transmitters $(s=$ $1,2)$ and the subsurfaces of any reflector and as shown in Figure. 2.5 is given by

$$
\mathbf{A}=\left[\begin{array}{cc}
h_{11} & h_{12} \\
h_{21} & h_{22} \\
\cdots & \cdots \\
h_{N 1} & h_{N 2}
\end{array}\right]
$$

Each element $h_{i s}$ in the matrix $\mathbf{A}$ is given by:

$$
h_{i s}(t)=G_{i s} \delta\left(t-\tau_{i s}\right) \quad s=1,2 \text {, and } i=1,2, \ldots, N
$$

The gain factor between the transmitter and any subsurface $G_{i s}$ is given by [51]:

$$
G_{i s}=\frac{\cos \left(\phi_{i s}\right) \cos \left(\theta_{i s}\right) A_{i}}{\pi R_{i s}^{2}}
$$

where $\phi_{i s}$ denotes the emitting angle between source $s$ and reflector subsurface $i, \theta_{i s}$ denotes the incident angle between source $s$ and reflector subsurface $i, R_{i s}$ denotes the distance between source $s$ and reflector subsurface $i$, and $A_{i}$ denotes the effective area of the reflector subsurface $i$.

The transfer function of (2.15) $H_{i s}(\omega)$ is given by (2.13), with $r$ replaced by $i$.

Assuming a high order reflection system, i.e. the transmitter $s$ emits the light to the reflector 1, which then reflects it to reflector 2 and from 2 to 3 and so on. This process 


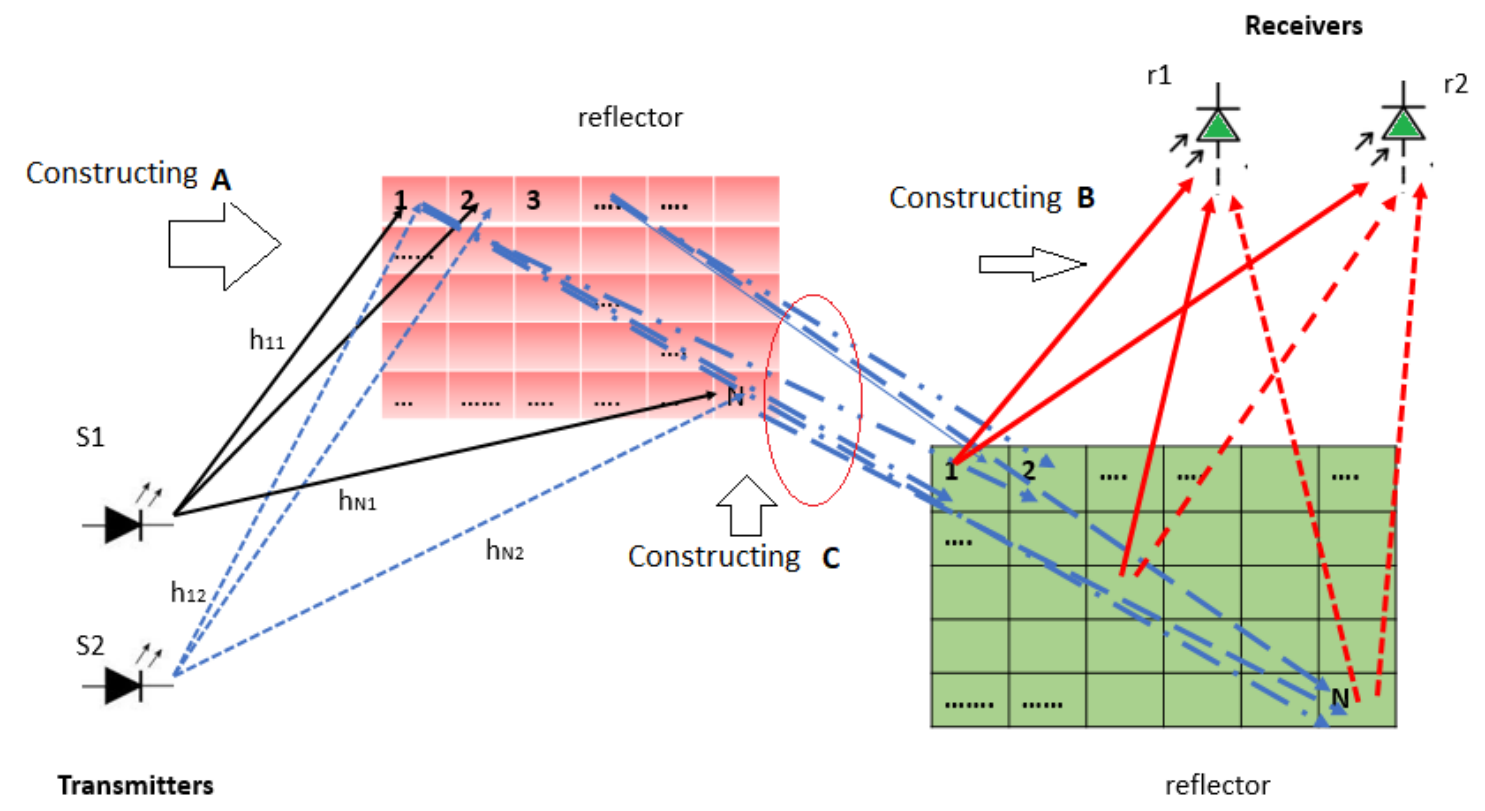

Figure 2.5: Illustration of how the NLOS path's matrices ( A, B, and $\mathbf{C}$ ) are constructed.

repeats until the last reflector radiates the power to the receiver $r$ directly. In every reflection, the optical power is reduced by the reflectivity factor $\rho_{i}$.

The impulse response matrix $\mathbf{B}$ between the receiver $r(r=1,2)$, and the last reflector (who has $\mathrm{N}$ subsurfaces) is given by

$$
\mathbf{B}=\left[\begin{array}{llll}
h_{11} & h_{12} & \ldots & h_{1 N} \\
h_{21} & h_{22} & \ldots & h_{2 N}
\end{array}\right]
$$

The gain factor $G_{r i}$, and the transfer function $H_{r i}(\omega)$ of each element in (2.17) are given by (2.11) and (2.13), respectively, but with the proper replacements of $i$ instead of $s$.

The corresponding impulse response matrix between any two reflectors (each with $\mathrm{N}$ subsurfaces) $\mathbf{C}$ is 


$$
\mathbf{C}=\left[\begin{array}{ccccc}
h_{11} & h_{12} & \ldots & \ldots & h_{1 N} \\
h_{21} & h_{22} & \ldots & \ldots & h_{2 N} \\
\ldots & \ldots & \ldots & \ldots & \ldots \\
h_{N 1} & h_{N 2} & \ldots & \ldots & h_{N N}
\end{array}\right]
$$

where $h_{k i}$, and the gain $G_{k i}$, are given by (2.11) and (2.12), respectively $(i, k=1,2, \ldots, N,(i$ and $k)$ are the subsurface's index of the first and second reflector respectively. Also, the transfer function $H_{k i}(\omega)$ is given by (2.13), after proper replacement of $k$ with $r$, and $i$ with $s$. Figure. 2.5 illustrates how the NLOS path's matrices (A, B, and $\mathbf{C})$ are constructed.

The total impulse response $h_{N L O S}(t)$ for the NLOS components is the sum of the contributions from the reflections up to a given order $L[52]$

$$
h_{N L O S}(t) \approx \sum_{l=1}^{L} h_{N L O S}^{(l)}(t)
$$

As an example, the first order reflection system (i.e. one reflector with $\mathrm{N}$ subsurfaces $(i=$ $1,2, \ldots, N)$ has an impulse response given by [51]

$$
h_{N L O S}^{(1)}(t)=\sum_{i=1}^{N} \rho_{i} h_{r i}(t) \otimes h_{i s}(t)
$$

The second order reflection system (i.e. two reflectors with $\mathrm{N}$ subsurfaces each $(i, k=$ $1,2, \ldots, N)$ is given by

$$
h_{N L O S}^{(2)}(t)=\sum_{i=1}^{N} \sum_{k=1}^{N} \rho_{k} \rho_{i} h_{k r}(t) \otimes h_{k i}(t) \otimes h_{i s}(t)
$$

The third order reflection system (i.e. three reflectors with $\mathrm{N}$ subsurfaces each $(i, k, l=$ 
$1,2, \ldots, N),(l$ is the subsurface's index of the third reflector) is given by

$$
h_{N L O S}^{(3)}(t)=\sum_{l=1}^{N} \sum_{i=1}^{N} \sum_{k=1}^{N} \rho_{k} \rho_{i} \rho_{l} h_{k r}(t) \otimes h_{l i}(t) \otimes h_{k l}(t) \otimes h_{i s}(t)
$$

and so on.

Many previous works such as [53] and [54] used recursive and iterative formulas to approximate (2.19). By the end, they got an exponential decay model for the VLC outdoor environment which is given by

$$
h_{N L O S}(t, \tau)=\frac{1}{\tau} e^{\frac{-t}{\tau}}
$$

where the time constant $\tau=2 \tau_{R M S}$ and $\tau_{R M S}$ is the channel RMS delay spread.

\subsubsection{Derivation of NLOS Transfer Function}

The frequency domain version of (2.14) is given by

$$
\mathbf{A}(\omega)=\left[\mathbf{h}_{i 1}(\omega) \quad \mathbf{h}_{i 2}(\omega)\right]
$$

where $\mathbf{h}_{i 1}(\omega)$ and $\mathbf{h}_{i 2}(\omega)$ are two vectors that indicate the transfer functions of the first and second transmitter and the reflector subsurfaces, respectively.

Any vectors in (2.24) can be expanded to

$$
\mathbf{h}_{i s}(\omega)=\left[\begin{array}{lll}
G_{1 s} e^{-j \omega \tau_{1 s}} & G_{2 s} e^{-j \omega \tau_{2 s}} & \left.\ldots G_{N s} e^{-j \omega \tau_{N s}}\right)
\end{array}\right]^{T} \quad s=1,2
$$


Similarly, the frequency domain version of (2.17) is given by

$$
\left.\mathbf{B}(\omega)^{T}=\left[\mathbf{h}_{1 i}(\omega) \quad \mathbf{h}_{2 i}(\omega)\right)\right]
$$

where $\mathbf{h}_{r i}(\omega)$, and $\mathbf{h}_{2 i}(\omega)$ are two vectors that indicate the transfer functions of the first and second receiver with the last reflector surface.

The vectors in (2.26) can be expanded to

$$
\mathbf{h}_{r i}(\omega)^{T}=\left[\begin{array}{lll}
G_{r 1} e^{-j \omega \tau_{r 1}} & \left.G_{r 2} e^{-j \omega \tau_{r 2}} \ldots G_{r N} e^{-j \omega \tau_{r N}}\right)
\end{array}\right]^{T} \quad r=1,2
$$

The $N \times N$ frequency domain version of (2.18) can be written as

$$
\mathbf{C}(\omega)=\left[\begin{array}{cccc}
G_{11} e^{-j \omega \tau_{11}} & G_{12} e^{-j \omega \tau_{12}} & . . & G_{1 N} e^{-j \omega \tau_{1 N}} \\
G_{21} e^{-j \omega \tau_{21}} & G_{22} e^{-j \omega \tau_{22}} & . . & G_{2 N} e^{-j \omega \tau_{2 N}} \\
. & & . . & . . \\
G_{N 1} e^{-j \omega \tau_{N 2}} & G_{N 2} e^{-j \omega \tau_{N 2}} & . & G_{N N} e^{-j \omega \tau_{N N}}
\end{array}\right]
$$

To include the reflectivity factors $\rho_{i}$ of the surfaces, we define the $N \times N$ reflectivity matrix as follows

$$
\mathbf{G}_{\rho}=\operatorname{diag}\left[\rho_{1} \rho_{2} \ldots \rho_{N}\right]
$$

In this work, we assume all the reflection coefficients are equal $\left(\rho_{1}=\rho_{2}=\ldots=\rho_{N}\right)$.

To have a closed form expression for the NLOS link transfer function, we have to find the FFT of (2.20), (2.21) and (2.22) till $l^{\text {th }}$ reflection order, then applying Carl Neumann's 
result on matrices as follows, for first order reflection

$$
\mathbf{H}^{(\mathbf{1})}{ }_{N L O S}(\omega)=\mathbf{B}(\omega) \mathbf{G}_{\rho} \mathbf{A}(\omega)
$$

For second-order reflection

$$
\mathbf{H}^{(2)}{ }_{N L O S}(\omega)=\mathbf{B}(\omega) \mathbf{G}_{\rho} \mathbf{C}(\omega) \mathbf{G}_{\rho} \mathbf{A}(\omega)
$$

For third order reflection

$$
\mathbf{H}^{(\mathbf{3})}{ }_{N L O S}(\omega)=\mathbf{B}(\omega) \mathbf{G}_{\rho}\left(\mathbf{C}(\omega) \mathbf{G}_{\rho}\right)^{2} \mathbf{A}(\omega)
$$

For the $l^{\text {th }}$ order reflection

$$
\mathbf{H}^{(1)}{ }_{N L O S}(\omega)=\mathbf{B}(\omega) \mathbf{G}_{\rho}\left(\mathbf{C}(\omega) \mathbf{G}_{\rho}\right)^{l-1} \mathbf{A}(\omega)
$$

The total NLOS transfer function is given by the infinite series

$$
\begin{aligned}
\mathbf{H}_{N L O S}(\omega) & =\sum_{l=1}^{\infty} \mathbf{H}_{N L O S}^{(l)}(\omega) \\
& =\mathbf{H}^{(\mathbf{1})}{ }_{N L O S}(\omega)+\mathbf{H}^{(\mathbf{2})}{ }_{N L O S}(\omega)+\mathbf{H}^{(\mathbf{3})}{ }_{N L O S}(\omega)+\ldots+\mathbf{H}^{(1)}{ }_{N L O S}(\omega)+\ldots \\
& =\mathbf{B}(\omega) \mathbf{G}_{\rho} \mathbf{A}(\omega)+\mathbf{B}(\omega) \mathbf{G}_{\rho} \mathbf{C}(\omega) \mathbf{G}_{\rho} \mathbf{A}(\omega)+\mathbf{B}(\omega) \mathbf{G}_{\rho}\left(\mathbf{C}(\omega) \mathbf{G}_{\rho}\right)^{2} \\
& \mathbf{A}(\omega)+\ldots+\mathbf{B}(\omega) \mathbf{G}_{\rho}\left(\mathbf{C}(\omega) \mathbf{G}_{\rho}\right)^{l-1} \mathbf{A}(\omega)+\ldots \\
& =\mathbf{B}(\omega) \mathbf{G}_{\rho} \sum_{m=0}^{\infty}\left(\mathbf{C}(\omega) \mathbf{G}_{\rho}\right)^{m} \mathbf{A}(\omega)
\end{aligned}
$$

Applying Carl Neumann's result on matrices $\left(\left(\mathbf{I}_{\mathbf{n}}-\mathbf{F}\right)^{-\mathbf{1}}=\sum_{\mathbf{m}=\mathbf{0}}^{\infty} \mathbf{F}^{\mathbf{m}}\right)$, then, the expression in (2.34) can be summed up to 


$$
\sum_{m=0}^{\infty}\left(\mathbf{C}(\omega) \mathbf{G}_{\rho}\right)^{m}=\left(\mathbf{I}-\mathbf{C}(\omega) \mathbf{G}_{\rho}\right)^{-1}
$$

where $\mathbf{I}$ denotes the unity matrix.

Rewrite (2.34) to get the final expression for the NLOS transfer function which is given by the matrix product as

$$
\mathbf{H}_{N L O S}(\omega)=\mathbf{B}(\omega) \mathbf{G}_{\rho}\left(\mathbf{I}-\mathbf{C}(\omega) \mathbf{G}_{\rho}\right)^{-1} \mathbf{A}(\omega)
$$

Equation (2.36) gives a closed form expression for the NLOS path, where the infinite number of reflections shown by (2.34) becomes not an issue.

The impulse response $h_{N L O S}(t)$ can then be obtained by performing Inverse Fast Fourier Transform (IFFT) on the transfer function $\mathbf{H}_{\mathrm{NLOS}}(\omega)$. Therefore, the total impulse response of the suggested model $h(t)$ can then be obtained by performing IFFT on the transfer function given by $(2.9)$.

\subsection{Performance Analysis of the V2V-VLC Channel Model}

\subsubsection{The Received Optical Power and SNR}

The total received optical power $P_{r}$ can be calculated as follows:

$$
P_{r}=P_{r}^{L O S}+P_{r}^{N L O S}
$$


The received optical power from the LOS path is given by

$$
P_{r}{ }^{\operatorname{LOS}}= \begin{cases}\left(\sum_{r=1}^{2} \sum_{s=1}^{2} \frac{I_{s}\left(\alpha_{s}, \beta_{s}\right) A_{r}}{(L E R) R_{r s}^{2}} \cos \left(\phi_{r s}\right) \cos \left(\theta_{r s}\right)\right) & \text { if } 0 \leq \theta_{r s} \leq \Psi_{r s} \\ 0, & \text { if } \theta_{r s}>\Psi_{r s}\end{cases}
$$

where $I_{s}\left(\alpha_{s}, \beta_{s}\right)$ is the luminous intensity of the transmitter $s$ from the direction $\left(\alpha_{s}, \beta_{s}\right)$ and its measured in candela $(\mathrm{cd}),\left(\alpha_{s}, \beta_{s}\right)$ are the vertical and horizontal angles of the headlight respectively, $\Psi$ is the receiver's FOV, $L E R$ is luminous efficacy of radiation of a high power LED.

The received optical power from the NLOS paths is given by

$$
P_{r}{ }^{N L O S}= \begin{cases}\left(\sum_{r=1}^{2} \sum_{s=1}^{2} \sum_{q=1}^{Q} \frac{I\left(\alpha_{s}, \beta_{s}\right) \cos \left(\theta_{q r}\right) A_{q} A_{r}}{(L E R) R_{q s}^{2}\left(R_{r q}^{2}+h_{i}^{2}\right)}\right. & \\ \left.\rho_{q} \cos \left(\phi_{r q}\right) \cos \left(\theta_{r q}\right)\right) & \text { if } 0 \leq \theta_{r q} \leq \Psi_{r q} \\ 0 & \text { if } \theta_{r q}>\Psi_{r q}\end{cases}
$$

where $Q$ is the total number of the reflectors, $\theta_{q s}$ denotes the incident angle between reflector $q$ and receiver $r, A_{q}$ denotes the reflector effective area, $h_{i}$ denotes the hight of the receiver, $R_{q s}$ denotes the distance between the transmitter and the reflector, $R_{r q}$ denotes the distance between the reflector and the receiver, $\phi_{r q}$ denotes the emission angle between the reflector and receiver, $\theta_{r q}$ denotes the incident angle between the receiver and the reflector, and $\rho_{q}$ denotes the reflection factor of reflector $q$. The illustration of these parameters can be seen in Figure. 2.4.

In this chapter, we assume the OOK modulation scheme and both transmitters have the same power. Thus, at the receiver, the SNR is given by [53], [55]

$$
S N R=\frac{\left(\gamma P_{r}\right)^{2}}{\sigma_{n}^{2}}
$$


where $\sigma_{n}^{2}$ is the noise power given by (1.7).

\subsubsection{Calculating the BER}

BER is the probability that an error may occur in a bit in the pulse train, i.e., a "1" bit turns into a "0" bit or vice versa.

$$
B E R=P(0) P(e / 0)+P(1) P(e / 1)
$$

where $P(0)$ and $P(1)$ are the probability of transmit "0" and "1" respectively, $P(e / 0)$ and $P(e / 1)$ are the conditional probability for receiving "1" while "0" was transmitted and receiving "0" while "1" was transmitted respectively.

In this work, we considered OOK-NRZ transmission and random noise Gaussian distribution. Moreover, we assumed both symbols have same variances and they have same probability to be transmitted (equally likely) i.e. $P(0)=P(1)=1 / 2$, in this case, the threshold level is set to be the halfway between the symbols, i.e at $V_{m} / 2$ as shown in Figure. 2.6.

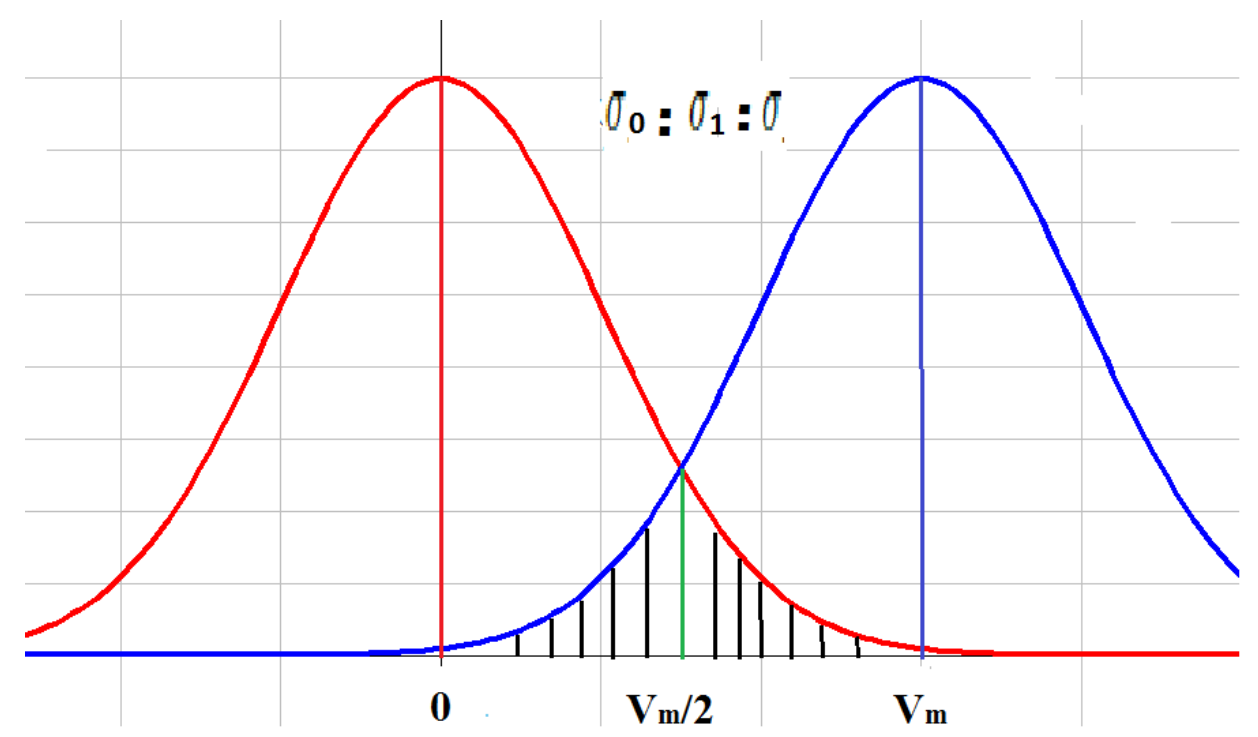

Figure 2.6: Conditional probability density function with OOK modulation

The conditional probability functions can be calculated as 


$$
\begin{aligned}
& P(e / 1)=\frac{1}{\sigma \sqrt{2 \pi}} \int_{-\infty}^{\frac{V_{m}}{2}} e^{\frac{-\left(x-\mu_{1}\right)^{2}}{2 \sigma^{2}}} d x \\
& P(e / 0)=\frac{1}{\sigma \sqrt{2 \pi}} \int_{\frac{V_{m}}{2}}^{\infty} e^{\frac{-\left(x-\mu_{0}\right)^{2}}{2 \sigma^{2}}} d x
\end{aligned}
$$

where $\mu_{0}=0$ and $\mu_{1}=V_{m}$ for NRZ signal.

substituting (2.42) and (2.43) in (2.41)

$$
\begin{aligned}
B E R_{O O K} & =\frac{1}{2 \sigma \sqrt{2 \pi}}\left(\int_{-\infty}^{\frac{V_{m}}{2}} e^{\frac{-\left(x-V_{m}\right)^{2}}{2 \sigma^{2}}} d x+\int_{\frac{V_{m}}{2}}^{\infty} e^{\frac{-(x-0)^{2}}{2 \sigma^{2}}} d x\right) \\
& =\frac{1}{2}\left(\frac{1}{2} \operatorname{erfc}\left(\frac{1}{2 \sqrt{2}} \sqrt{S N R}\right)+\frac{1}{2} \operatorname{erfc}\left(\frac{1}{2 \sqrt{2}} \sqrt{S N R}\right)\right) \\
& =\frac{1}{2} \operatorname{erfc}\left(\frac{1}{2 \sqrt{2}} \sqrt{S N R}\right) \\
& =\frac{1}{2} \operatorname{erfc}\left(\frac{1}{2 \sqrt{2}} \sqrt{\frac{\left(\gamma P_{r}\right)^{2}}{\sigma_{n}^{2}}}\right)
\end{aligned}
$$

where $\operatorname{erfc(.)}$ is the complementary error function used to calculate the tail probability of the standard Gaussian distribution, its given by

$$
\operatorname{erfc}(x)=\frac{2}{\sqrt[2]{\pi}} \int_{x}^{\infty} e^{-y^{2}} \mathrm{~d} y
$$

\subsubsection{Time Dispersion Parameters for the Channel}

By using the derived CIR given by (2.8), we can calculate several channel parameters such as RMS delay spread, mean excess delay, frequency correlation function, coherence time, and coherence bandwidth. 
The RMS delay spread and mean excess delay are given by [56]

$$
\tau_{R M S}=\sqrt{\frac{\int_{0}^{\infty}(t-\bar{\tau})^{2} h^{2}(t) d t}{\int_{0}^{\infty} h^{2}(t) d t}}
$$

where $\bar{\tau}$ is the mean excess delay and it's given by

$$
\bar{\tau}=\frac{\int_{0}^{\infty} t h^{2}(t) d t}{\int_{0}^{\infty} h^{2}(t) d t}
$$

For V2V-VLC environment, the frequency correlation function $H(\triangle \omega)$ can be obtained from the CIR as $[57]$

$$
H(\triangle \omega)=\int_{-\infty}^{\infty} h(t) e^{-j \Delta \omega t} d t
$$

Once the frequency correlation function is calculated, the coherence bandwidth $B_{c}$ for a VLC channel can be found as [58]

$$
B_{c}=|\min \triangle(\omega)|
$$

Since V2V-VLC system is good for short distance communications. The effective velocity of both the transmitter and the receiver $\left(V_{e f f}=\sqrt{V_{s}^{2}+V_{r}^{2}}\right)$ compared to the velocity of light is very small, thus, a correlation level of 0.9 is typically chosen [58], i.e $|H \triangle(\omega)|=0.9$. 
Table 2.1: Simulation parameters

\begin{tabular}{|c|c|}
\hline \hline Parameter & Value \\
\hline Number of reflectors & $2-6$ \\
\hline Modulation type & OOK \\
\hline Distance between the reflectors & $3-80 \mathrm{~m}$ \\
\hline Distance between the transmitter and the receiver & $20-120 \mathrm{~m}$ \\
\hline Receiver area & $4 \mathrm{~cm}^{2}$ \\
\hline Total area of the reflector & $0.5 \mathrm{~m}^{2}$ \\
\hline LED luminance intensity & $1000-2500 \mathrm{~cd}$ \\
\hline Transmitted and received angles & $5-45^{\circ}$ \\
\hline Responsivity of PD $\gamma$ & $0.54 \mathrm{~A} / \mathrm{W}$ \\
\hline Reflectivity $\rho$ & 0.8 \\
\hline Gain of the optical filter T( $\Psi)$ & 1 \\
\hline Gain of the optical concentrator $\mathrm{g}$ & 5 \\
\hline Frequency correlation level & 0.9 \\
\hline Speeds of the transmitter and receiver, respectively & $30 \mathrm{Km} / \mathrm{h}, 25 \mathrm{Km} / \mathrm{h}$ \\
\hline Data rate & $35 \mathrm{Mbps}$ \\
\hline Receiver hight & $70 \mathrm{~cm}$ \\
\hline
\end{tabular}

\subsection{Simulation and Results}

MATLAB software is used to simulate the V2V-VLC system. The simulation parameters are shown in Table. 1.2, and Table. 2.1. Multi-path propagation is considered. We assume all reflector surfaces to have the same reflectively factor and same areas. We consider the moving speed of $30 \mathrm{Km} / \mathrm{h}$ for the transmitter and $25 \mathrm{Km} / \mathrm{h}$ for the receiver( whether they move in the same direction, or in the opposite direction). We use Philips Ultinon LED 12985BWX2 as transmitter [59]. Also, we use APD model (Si) Hamamatsu $S 8664-1010$ as a receiver [21]. We analyze the system performance considering various parameters.

Figure. 2.7 shows the CIR of the LOS and the NLOS components when the transmitter and the receiver are moving in the same directions, but with different velocities. The CIR of the LOS path is represented by an impulse, while the CIR of the NLOS components has an exponential decay behavior. Moreover, Figure. 2.7 shows that, as the distance between the transmitter and the receiver increases, the delay increases but the channel gain decreases. The low gain shown in Figure. 2.7 for both paths is due to the nature of V2V-VLC system where the channel is changing very fast. In 

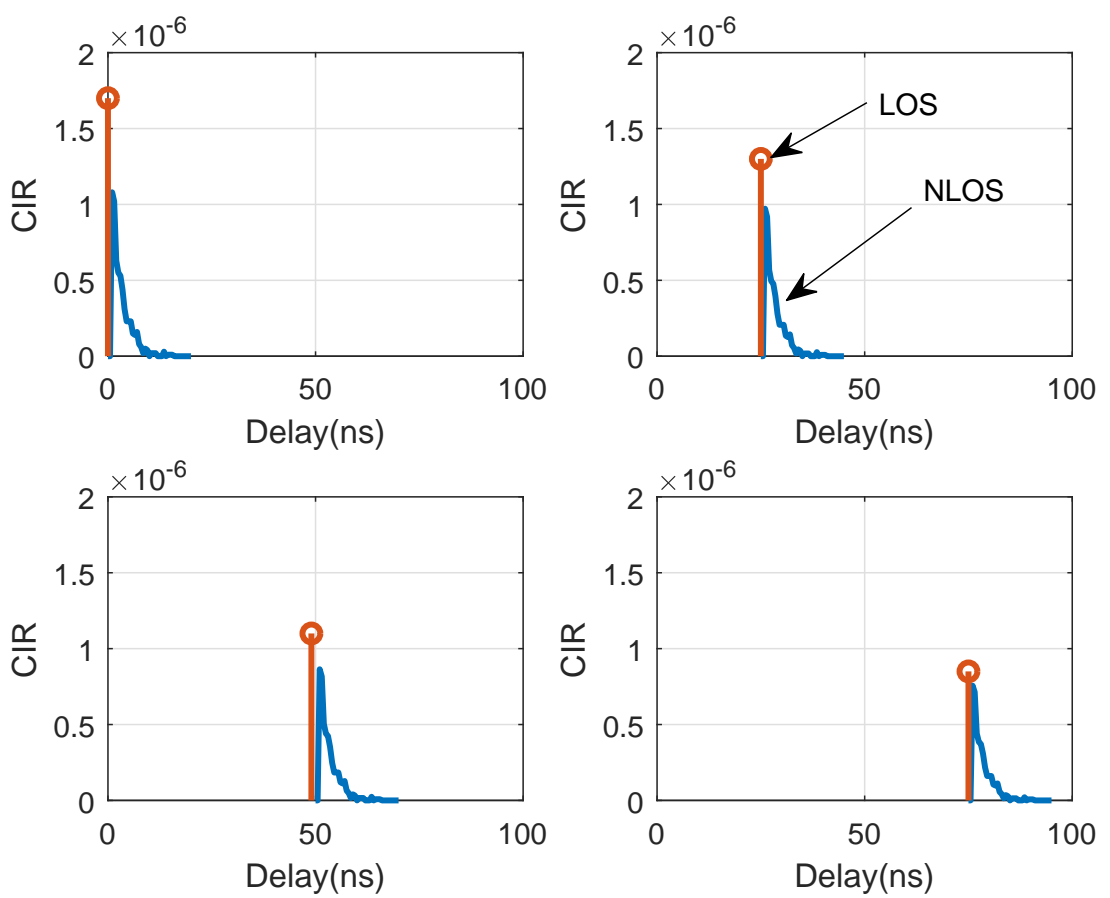

Figure 2.7: Channel impulse response of the LOS and NLOS components when vehicles are moving in the same direction.

addition, both the transmitter and the receiver are moving affecting both the angle of emitting, as well as, the angles of incidence. The importance of the channel gain in the V2V-VLC system rising from it determines the achievable SNR for fixed transmitting power. Figure. 2.8 shows the transfer function of the V2V-VLC channel. We use (2.36) to simulate the NLOS paths for up to six reflectors, while we use (2.12) and (2.13) to simulate the gain of the LOS path. From Figure. 2.8, we see that the gain of the channel depends not only on frequency but also on other factors such as emitting angle, incident angle, and the reflectivity of the surrounding objects. Also, Figure. 2.8 shows the difference between LOS gain and NLOS gain is inversely proportional to a number of reflections. This is mean that, we need a high number of reflection to make the difference close to zero. The exponential decay of the CIR for the diffusion path shown in Figure. 2.7 is obtained after performing IFFT for the transfer function shown in Figure. 2.8. Smoothing operation has to be done for the transfer function (such as raised cosine window) before applying the IFFT. The simulation results show acceptable similarity with the mathematical model for CIR given by (2.23) as shown in Figure. 2.9. 


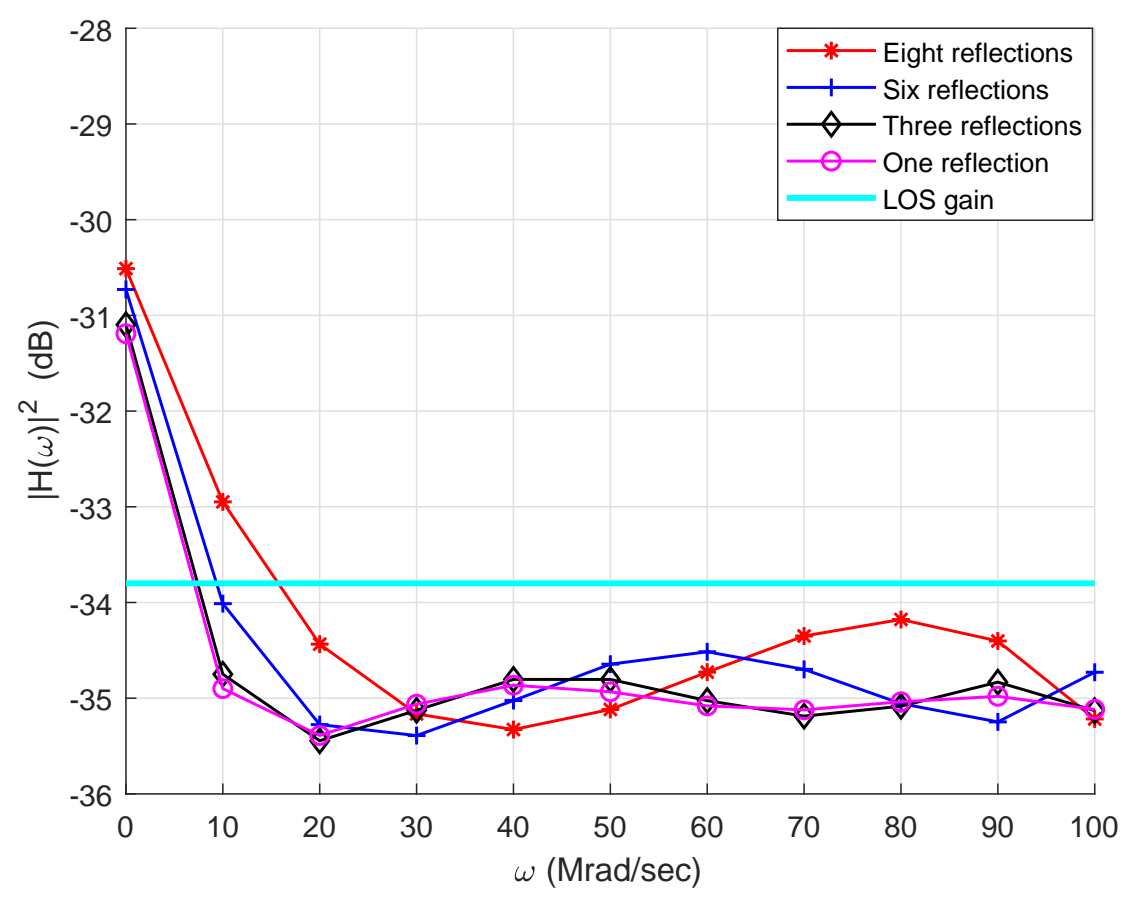

Figure 2.8: The LOS and NLOS paths transfer functions.

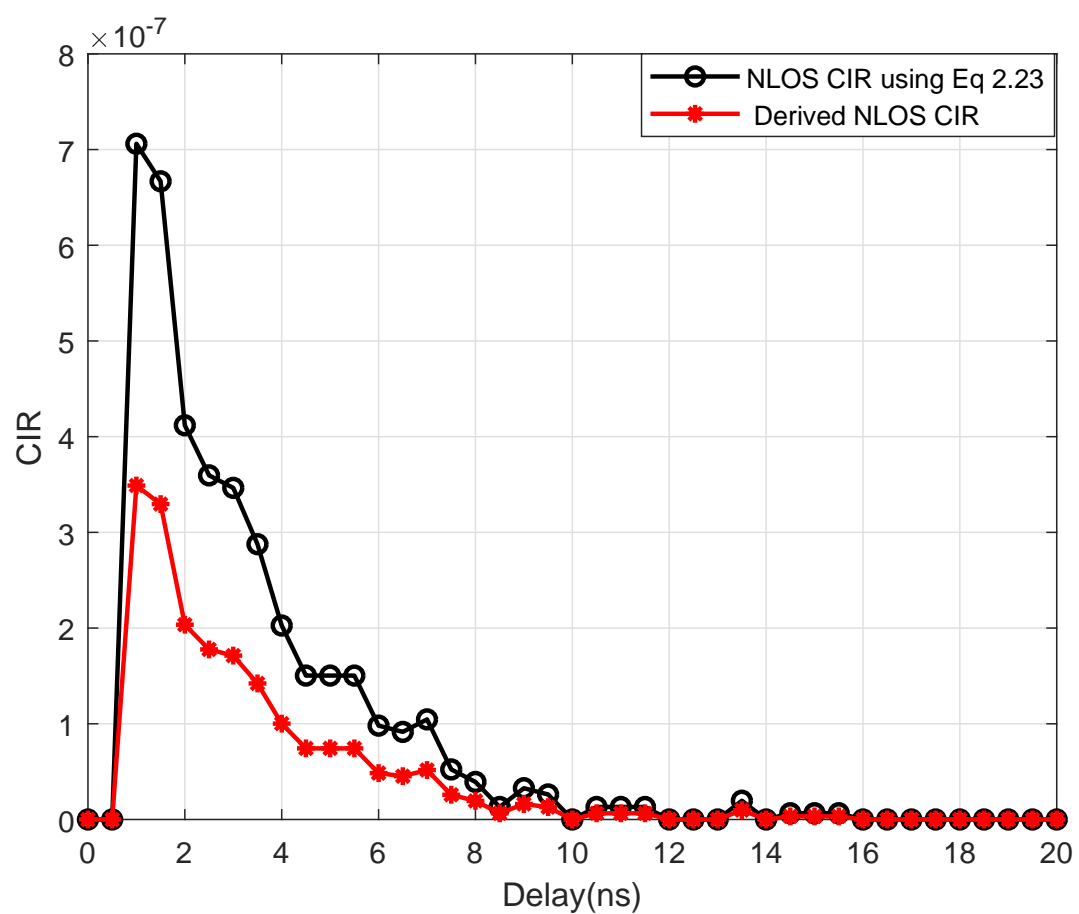

Figure 2.9: Comparison between derived NLOS CIR and NLOS CIR given by EQ (2.23). 


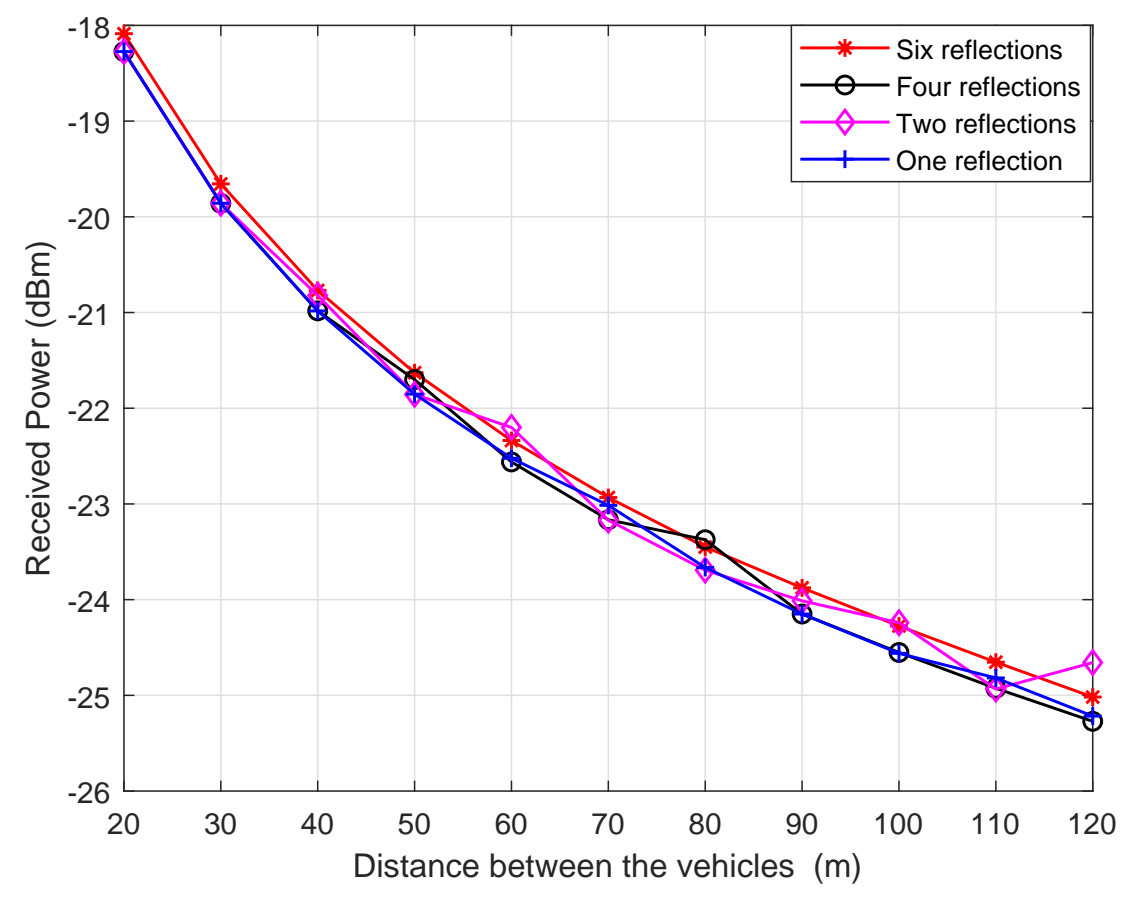

Figure 2.10: Received power versus the distance between vehicles.

Figure. 2.10 shows the received power versus the distance between the vehicles for up to six reflectors. The received power is inversely proportional to the distance. Furthermore, it can be seen that the contribution by the high order reflections $(l>2)$ are not dominant. The dominant components are coming from the LOS path as well as from the first and second reflections. This is because of the scattering property of the light and the absorption by the reflectors and other surrounding objects reduce the received power strength. BER versus different values for $R_{r s}$ at a data rate of $35 \mathrm{Mbps}$ is shown in Figure. 2.11. It can be seen that, as the distance increases, the system BER performance decreases. Also, we can see from Figure. 2.11 that, the value of the BER degrades from $10^{-6}$ at $R_{r s}=30 \mathrm{~m}$ to $3.5 \times 10^{-5}$ at $R_{r s}=80 \mathrm{~m}$. This happens because when $R_{r s}$ changes from 30 to $80 \mathrm{~m}$, the received optical power reduces by more than 3 times (e.g., it decreases from $-24 \mathrm{dBm}$ to $-20 \mathrm{dBm}$ ) according to Figure. 2.10.

The investigated model shows better performance than the results obtained in [7] and[40], since we consider the passing by, and parking cars as reflectors. Also, Figure. 2.11 emphasizes that the high order number of reflections will not cause significant difference in the performance of the system. 


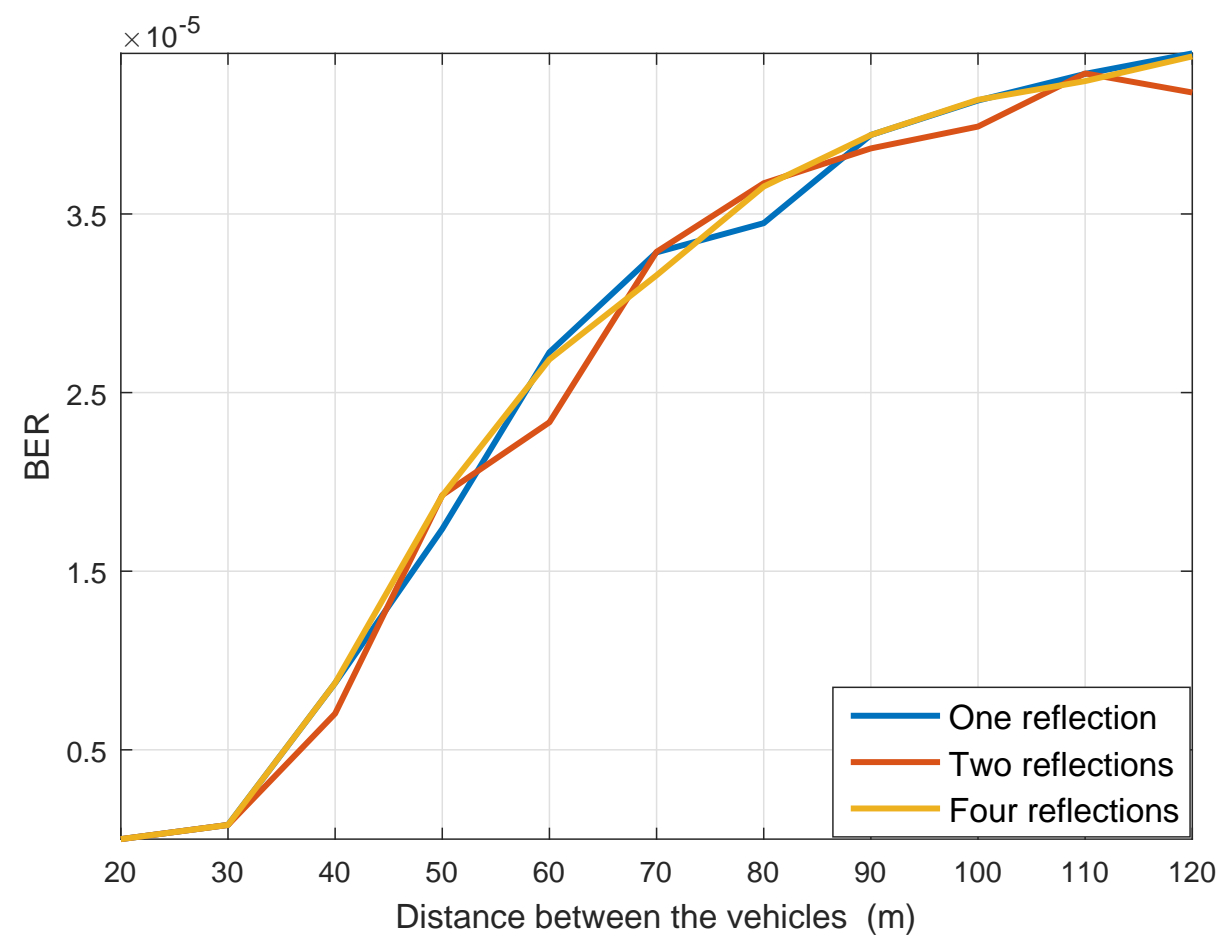

Figure 2.11: BER versus the distance between vehicles moving in same direction.

Figure. 2.12 shows the PDF of the delay spread for the V2V-VLC channel. It can be seen that the best fitted distribution for the RMS of the channel in the case of the same direction movement is similar to Rician distribution. The mean value of the delay spread of V2V-VLC model is about $12 \mathrm{~ns}$, while the authors in [16] found it to be 10-15 ns as shown in Table. 2.2. This is attributed to the fact that the distance is short while the signal speed is very high in V2V-VLC systems, which produce a dispersive channel. In addition, motion introduces additional Doppler spreading and shifting.

Table 2.2: RMS delay spread comparison

\begin{tabular}{|c|c|}
\hline \hline Reference & Delay Spread (ns) \\
\hline V2V $[16]$ & 10 \\
\hline V2I $[16]$ & 15 \\
\hline Infrared channels $[60]$ & 2 \\
\hline For this work when vehicles move in the same direction & 12 \\
\hline
\end{tabular}

Figure. 2.13, and Figure. 2.14 depict the average of $50 \%$ and $90 \%$ coherence time of the suggested channel when the transmitter and the receiver are moving in the same direction, and in 


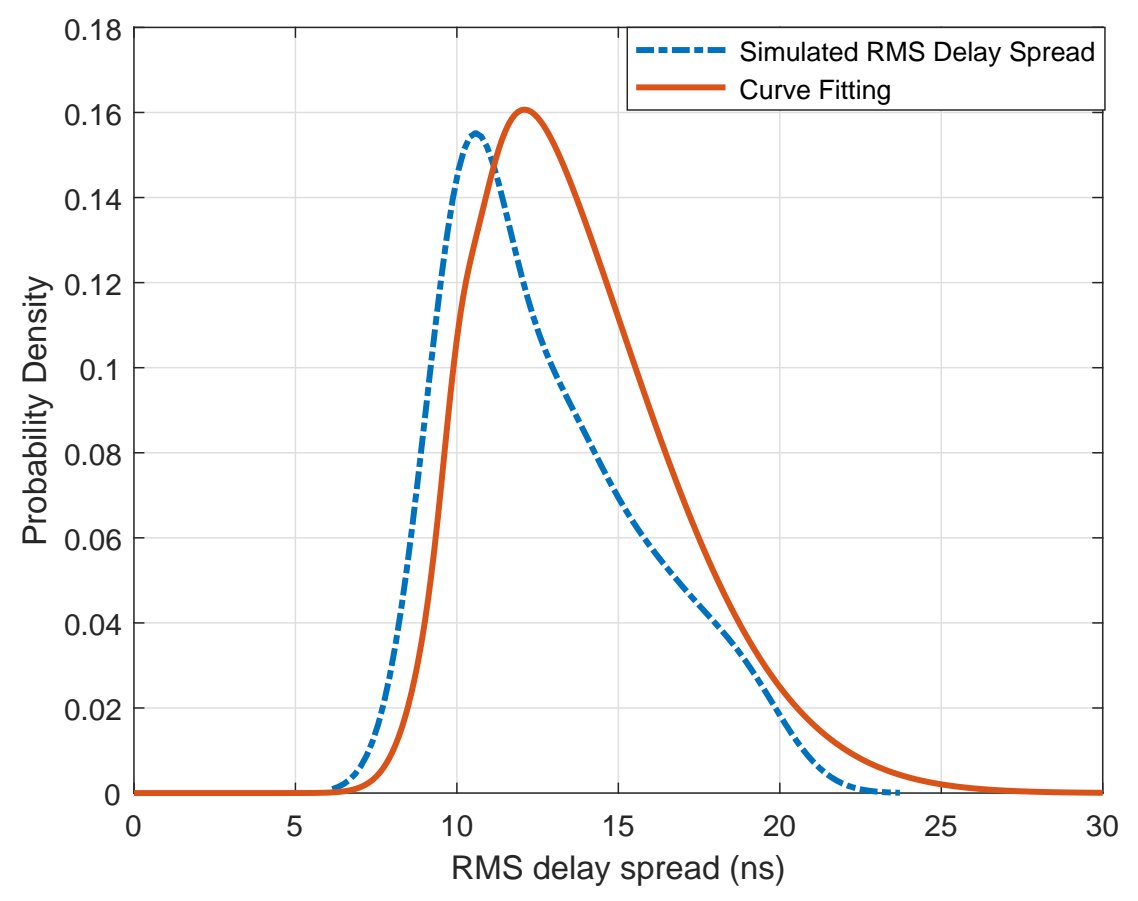

Figure 2.12: RMS delay spread for one reflection system.

opposite direction, respectively. There is a decreasing trend in coherence time with effective speed for both directions, as expected, which is consistent with the Doppler spread analysis. The VLC channel shows a larger coherence time than the values reported for a V2V-RF channel in the urban scenarios [61], [62]. Table. 2.3 compares the median coherence time of (VLC and RF) obtained from this work and several previous works. Moreover, the V2V-VLC model shows that $2 \times 2$ MIMO-VLC links have much slower channel time variation compared to RF V2V links, and other VLC models. Also, the V2V-VLC channel coherence time is found to be at least an order of magnitude larger than that of RF channels, indicating that the V2V-VLC channels are much stable.

Table 2.3: Median coherence time comparison (units in $(m s)$ )

\begin{tabular}{|c|c|c|}
\hline \hline Channel & $50 \%$ & $90 \%$ \\
\hline RF channel [62] & 103 & 3.6 \\
\hline VLC channel [63] & 164.7 & 46.8 \\
\hline VLC channel [42] & 367 & 33 \\
\hline For this work when vehicles move in the same direction & 220 & 50 \\
\hline For this work when vehicles move in the opposite direction & 100 & 25 \\
\hline
\end{tabular}




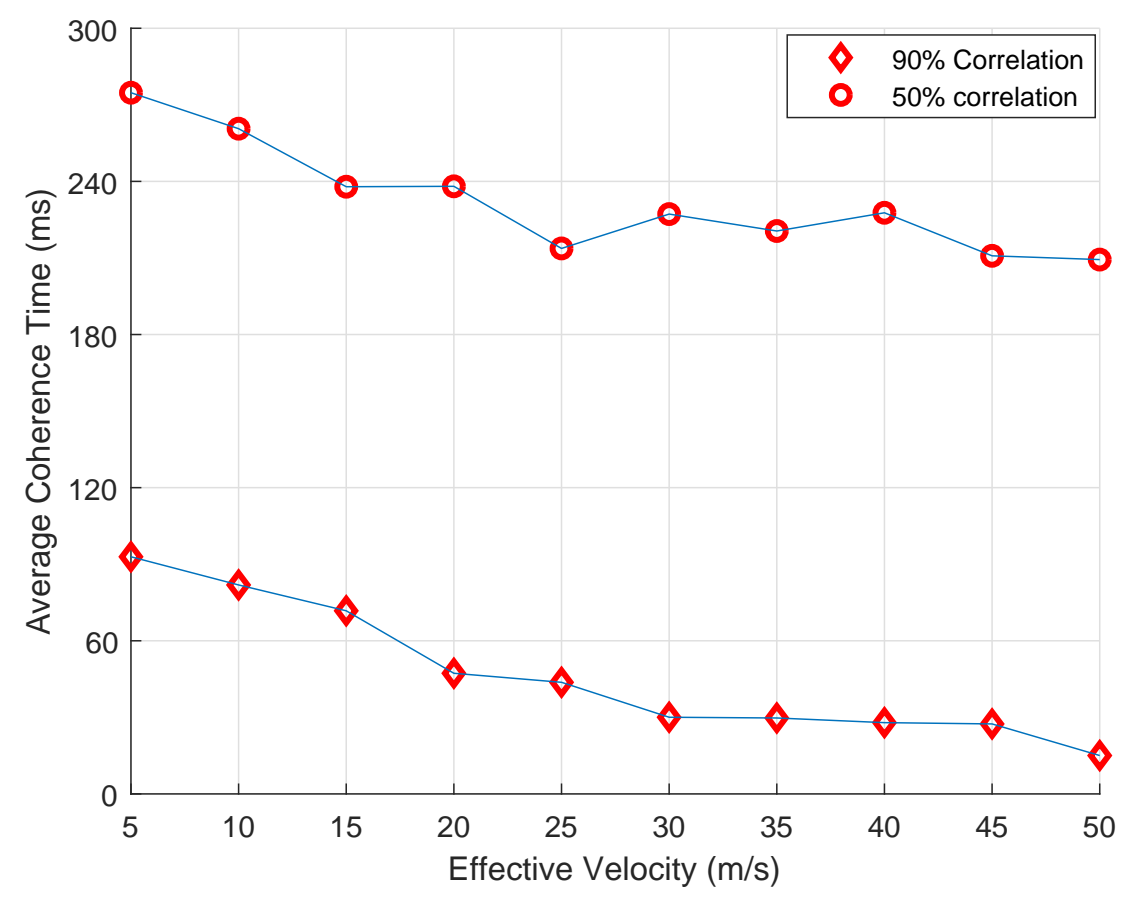

Figure 2.13: Channel coherence time when both vehicles move in the same direction.

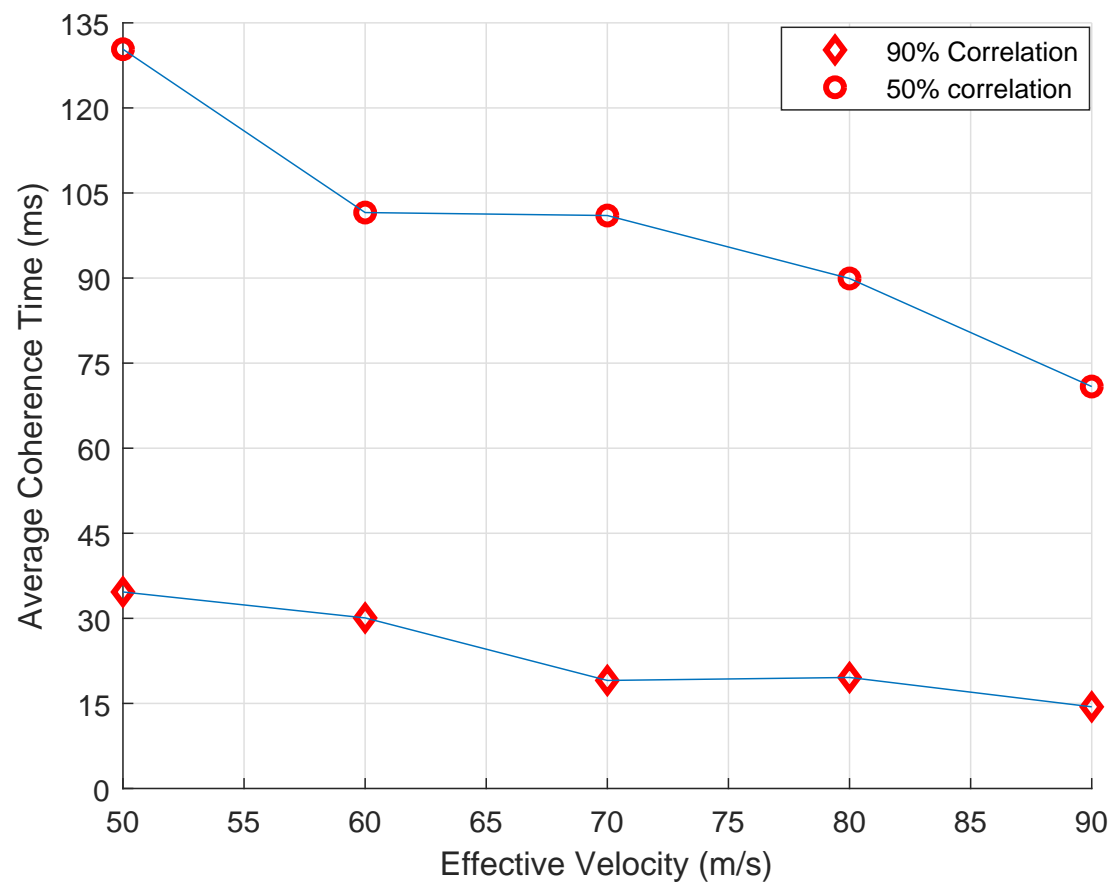

Figure 2.14: Channel coherence time when both vehicles move in the opposite direction. 


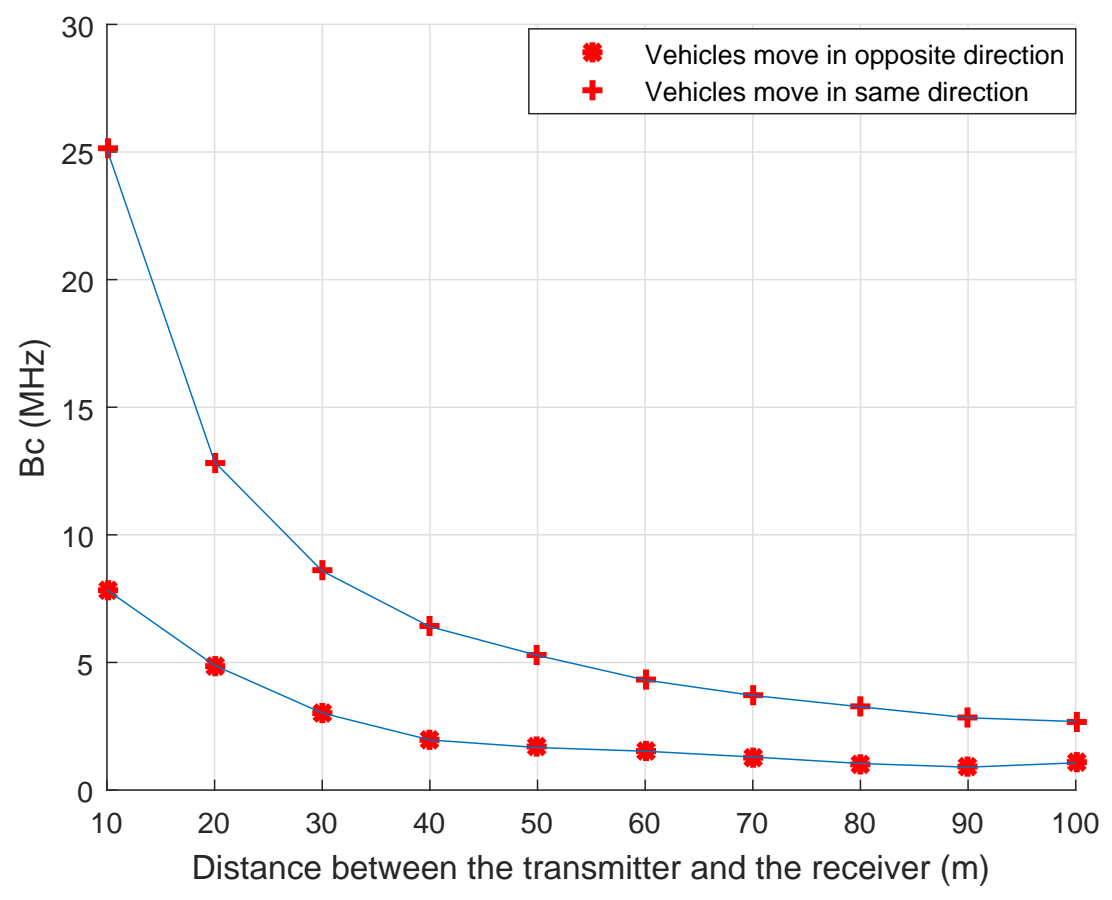

Figure 2.15: Coherence bandwidth for one reflection system with 0.9 correlation level when vehicles move in same and opposite direction.

In this work, the coherence time was computed directly from received signals instead of assuming a reciprocal time-frequency relationship. The fact that the coherence time of the VLC system is high, specially when both vehicles move in the same direction, is attributed to the very high frequency of light. The movement in the opposite direction appears to have lower channel coherence time than the movement in the same direction. This is because the effective speed of both vehicles will be higher, and the dominant path is the NLOS only. We can suggest that the V2V-VLC channel can be considered as a selective fading channel. Note that the simulated coherence time does not continue to decrease monotonically with distance since VLC signal strength varies rapidly, it is plausible that small perturbations in the environment could lead to relatively large changes in the channel gain.

The coherence bandwidth values as a function of separation distances are shown in Figure. 2.15, where the instantaneous coherence bandwidth is inversely proportional to the separation distance. The highest coherence bandwidth values are available for the lowest 
RMS delay spread values, and higher values can be seen for the same direction movement. The coherence bandwidth values range between 3-7 MHz for the opposite direction movement, and $4-25 \mathrm{MHz}$ for the same direction movement.

Low RMS delay spread values lead to higher coherence bandwidth because in the same direction movement, the NLOS path can be considered dominant. Also, this result shows the importance of the scattering environment of the channel, as the paths take less time to reach the receiver, so the coherence bandwidth of the channel increases.

\subsection{Chapter Summary}

In this chapter, we model the V2V-VLC communication system as $2 \times 2$ MIMO, considering the real-time environment, practical reflectors, and real ambient noise. Then, the channel of communication is modeled considering LOS and NLOS paths based on upcoming IEEE 802.15.7r1. We derive the CIR expressions for LOS and NLOS paths. Finally, we calculate the channel parameters such as BER, SNR, RMS delay spread, coherence bandwidth, and coherence time when the vehicles move in the same direction and when they move in the opposite direction. 


\section{Chapter 3}

\section{Performance Analysis of Adaptive}

\section{OFDM Modulation Scheme in VLC}

\section{Vehicular Communication Network}

VLC channels have inherent scattering and reflection properties and undergo destructive effects of multipath fading and frequency selective fading. This results in ISI and leads to a reduced data rate. In order to mitigate ISI, Time Domain Equalization (TDE) technique is used in single carrier and OFDM is used in multi-carrier communication systems [39]. OFDM has been implemented in many wireless RF and wireline standards and has also proved to be beneficial in VLC systems [9]. O-OFDM schemes have better optical power efficiency, reduced ISI and very low BER, compared to conventional optical modulation schemes such as OOK and Pulse Position Modulation (PPM) [64].

MIMO systems have gained considerable attention in VLC networks due to their high data rate capabilities over longer distances despite multipath fading and interference effects. The combination of OFDM and MIMO is considered as a powerful physical layer solution for high-speed vehicular VLC systems especially for accommodating bandwidth-hungry applications [65].

Previously, the combination of MIMO and OFDM has been mostly used for non-adaptive 
systems or for single modulation scheme. Few existing works have realized V2V-VLC systems as MIMO systems or used optical OFDM. Adaptive transmission in VLC has been explored before, but mostly for indoor applications [66]. Researchers have only assumed a Gaussian channel with a fixed modulation scheme, with an aim to increase the data rate.

In this chapter, we propose a novel VLC algorithm for $\mathrm{V} 2 \mathrm{~V}$ communication system using the channel model given in section 2.2. We use O-OFDM with adaptive modulation schemes to mitigate ISI, and also to improve data rate. We consider DCO-OFDM and ACO-OFDM schemes for our VLC system. We consider practical reflectors, sunlight as a main noise source, and the effect of clipping noise. Moreover, the performance of the V2V-VLC system is optimized by Singular Value Decomposition (SVD) technique. Also, we apply bit-loading algorithm to improve the performance of the system. We analyze our proposed algorithm with numerical simulation and show its superiority by performing a comparative analysis with conventional modulation schemes. Data rate of up to $50 \mathrm{Mbps}$ with reduced BER, is achieved.

\subsection{Related Work in Adaptive Modulation for VLC}

In literature, there has been limited research in O-OFDM-V2V-VLC systems. In this section, some of these works will be reviewed. Performance of VLC system using Space-Time Block Coding (STBC)-OFDM technique for ITS was investigated in [53]. They modeled the outdoor

optical channel as a Rician channel. They proposed STBC-OFDM coding to reduce the influence of multi-path dispersion associated with the channel to achieve BER of $10^{-6}$ even at a low SNR. The authors in [67] presented a Single-Input Single-Output (SISO)-V2V-VLC system with DCO-OFDM. They proposed a new automotive VLC system based on the Optical Communication Image Sensor (OCI) as a receiver. Then, they applied DCO-OFDM to achieve more flexible and effective signal transmission. Also, they considered the characteristics of the LED and OCI to select the appropriate parameter values for signal processing to achieve higher data rates. The authors in [68] introduced the concept of an environment-adaptive VLC receiver for a vehicular communication system in a visible light environment to mitigate 
the effects of problematic conditions such as supporting long-distance communications in dynamic traffic situations and in unfriendly atmospheric conditions. In [69], the authors suggested DCO-OFDM based MIMO transmission scheme for vehicular communication. They evaluated the performances of different MIMO modes such as spatial multiplexing, and repetition code with different modulation techniques and different transmitter-receiver selection. They found the spatial multiplexing technique suffers from channel correlation, while repetition code is unsuitable for high modulation orders.

\subsection{Optical OFDM Scheme of V2V-VLC System}

This section outlines the investigation scheme for V2V-VLC channel and analyzes it with various types of OFDM techniques. In this work, the model presented in section 2.2 and shown in Figure. 2.1 is modified to include O-OFDM with an adaptive modulation scheme. This reduces ISI and increases the data rate. Figure. 3.1 shows the block diagram of the proposed Optical OFDM scheme for V2V-VLC system.

In Figure. 3.1, a $\mathrm{S} / \mathrm{P}$ converter is used to split a binary source of data into two data streams $\mathbf{x}_{\mathbf{1}}, \mathbf{x}_{\mathbf{2}}$. Both streams are modulated using Quadrature Amplitude Modulation (QAM) and the resulting symbols are assigned to subcarriers. Afterward, IFFT is performed and data is converted back to a single stream using a Parallel-to-Serial $(\mathrm{P} / \mathrm{S})$ converter after adding a Cyclic Prefix (CP). Then, the signal is changed to unipolar using one of the O-OFDM schemes. Finally, the data is converted to analog signal $s(t)$ using a Digital-to-Analog (D/A) converter along with low pass filter (shaping filter).

\subsubsection{DCO-OFDM Modulation Scheme}

DCO-OFDM is a form of O-OFDM with a DC bias added. In such systems, the frequency domain signal is transformed into the time domain by using the inverse discrete Fourier transform, which can be implemented by using an IFFT. In this work, the length of the IFFT is assumed to be N. The modulated signal in the frequency domain must be conjugate 


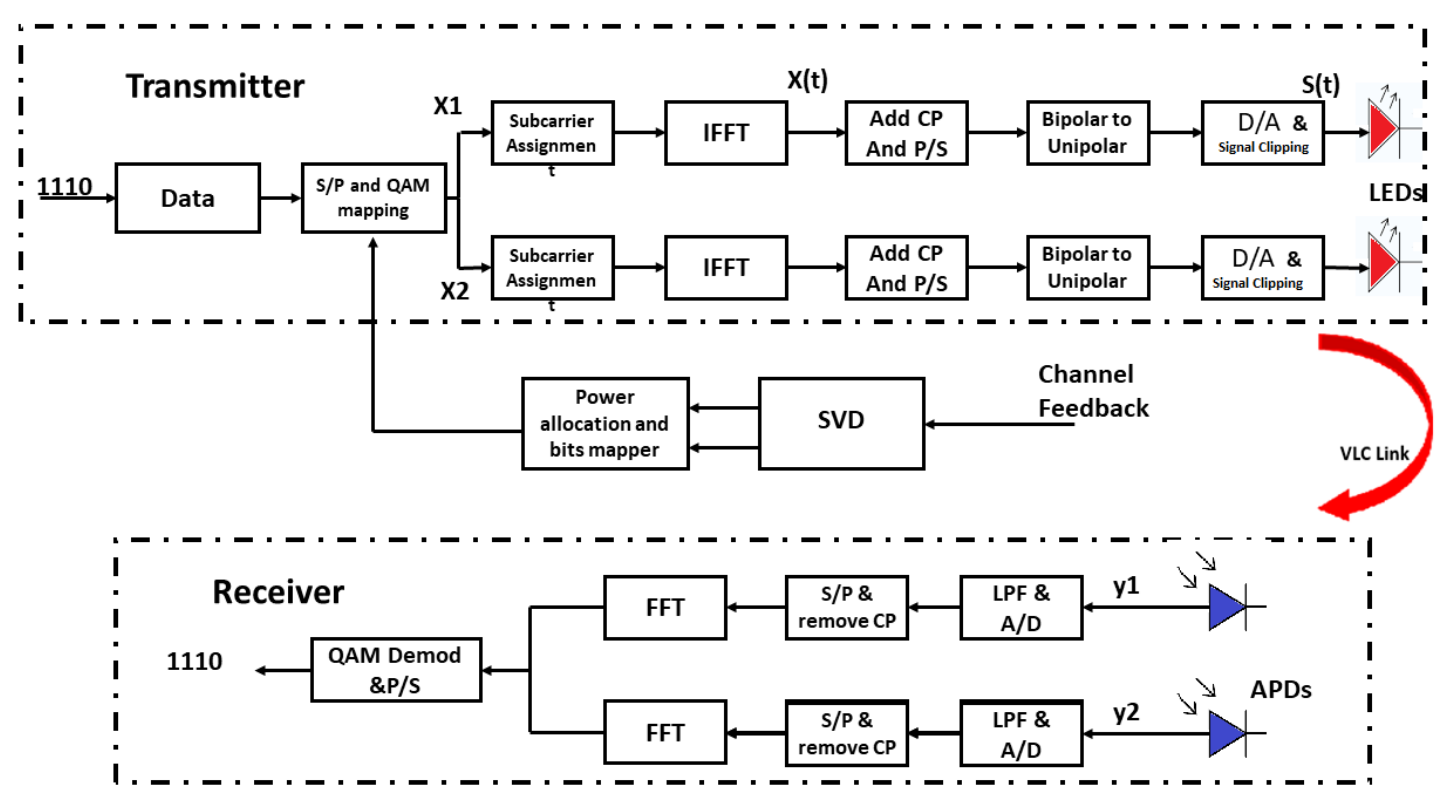

Figure 3.1: Simplified diagram of the V2V-VLC-OFDM scheme.

symmetric to ensure that the time domain signal is real. Moreover, a DC bias is added to guarantee that the transmitted signals are nonnegative.

In the frequency domain, the modulated signals satisfy the following conditions

$$
\left\{\begin{array}{l}
X(0)=X\left(\frac{N}{2}\right)=0 \\
X(K)=X^{*}(N-K)
\end{array} \quad K=1,2, \ldots, N-1\right.
$$

where $N$ is the size of IFFT, and $*$ is conjugate sign.

Thus, the input vector to the IFFT block in Figure. $3.1 \mathrm{x}$ can be given as

$$
\mathrm{x}=\left[\begin{array}{llllllllll}
0 & \mathrm{~s}_{1} & \mathrm{~s}_{2} & \ldots & \mathrm{s}_{\mathrm{N} / 2-1} & \mathbf{0} & \mathrm{s}_{\mathrm{N} / 2-1}^{*} & \ldots & \mathrm{s}_{2}^{*} & \mathrm{~s}_{1}^{*}
\end{array}\right]
$$


The time domain signal can be obtained by performing IFTT and it can be expressed as

$$
\begin{aligned}
x[n] & =\frac{1}{N} \sum_{K=0}^{N-1} X(K) e^{\frac{j 2 \pi K n}{N}} \\
& =\frac{1}{N} \sum_{K=0}^{\frac{N}{2}-1} X(K) e^{\frac{j 2 \pi K n}{N}}+\frac{1}{N} \sum_{\frac{N}{2}+1}^{N-1} X(K) e^{\frac{j 2 \pi K n}{N}} \\
& =\frac{1}{N} \sum_{K=1}^{\frac{N}{2}-1}\left(X(K) e^{\frac{j 2 \pi K n}{N}}+X^{*}(K) e^{\frac{-j 2 \pi K n}{N}}\right) \\
& =\frac{2}{N} \sum_{K=1}^{\frac{N}{2}-1}\left(a(K) \cos \left(\frac{2 \pi K n}{N}\right)-b(K) \sin \left(\frac{2 \pi K n}{N}\right)\right)
\end{aligned}
$$

It is assumed that $X(K)=a(K)+j b(k)$ is a zero mean complex random variable with variance $\sigma_{K}^{2}, X(K), K=1, \ldots, N / 2-1$, are independent, and $a(K)$ and $b(K)$ are independent real random variables with a zero mean and variance $\sigma_{K}^{2} / 2$.

According to the central limiting theorem, $\mathrm{x}[\mathrm{n}]$ is a Gaussian random variable with zero mean, that is $E[x(n)]=0$, where $E[$.$] stands for expectation.$

The variance of $\mathrm{x}[\mathrm{n}]$ can be derive as

$$
\begin{aligned}
\sigma_{x}^{2} & =E\left[x[n] x^{*}[n]\right] \\
& =\frac{4}{N^{2}} \sum_{K=1}^{\frac{N}{2}-1}\left(\frac{\sigma_{K}^{2}}{2} \cos ^{2}\left(\frac{2 \pi K n}{N}\right)+\frac{\sigma_{K}^{2}}{2} \sin ^{2}\left(\frac{2 \pi K n}{N}\right)\right) \\
& =\frac{2}{N^{2}} \sum_{k=1}^{N / 2-1} \sigma_{k}^{2}
\end{aligned}
$$

In DCO-OFDM technique, data is assigned to both odd and even subcarriers. A real signal can be then obtained by imposing the Hermitian symmetry property as shown in (3.1). This property implies that half of the OFDM subcarriers are utilized to generate the real-time domain signal [70].

The output of D/A converter is an analog real-time signal which is shown in Figure. 3.2 (a). This signal cannot be used to modulate the LEDs because of its bipolar nature. A certain 

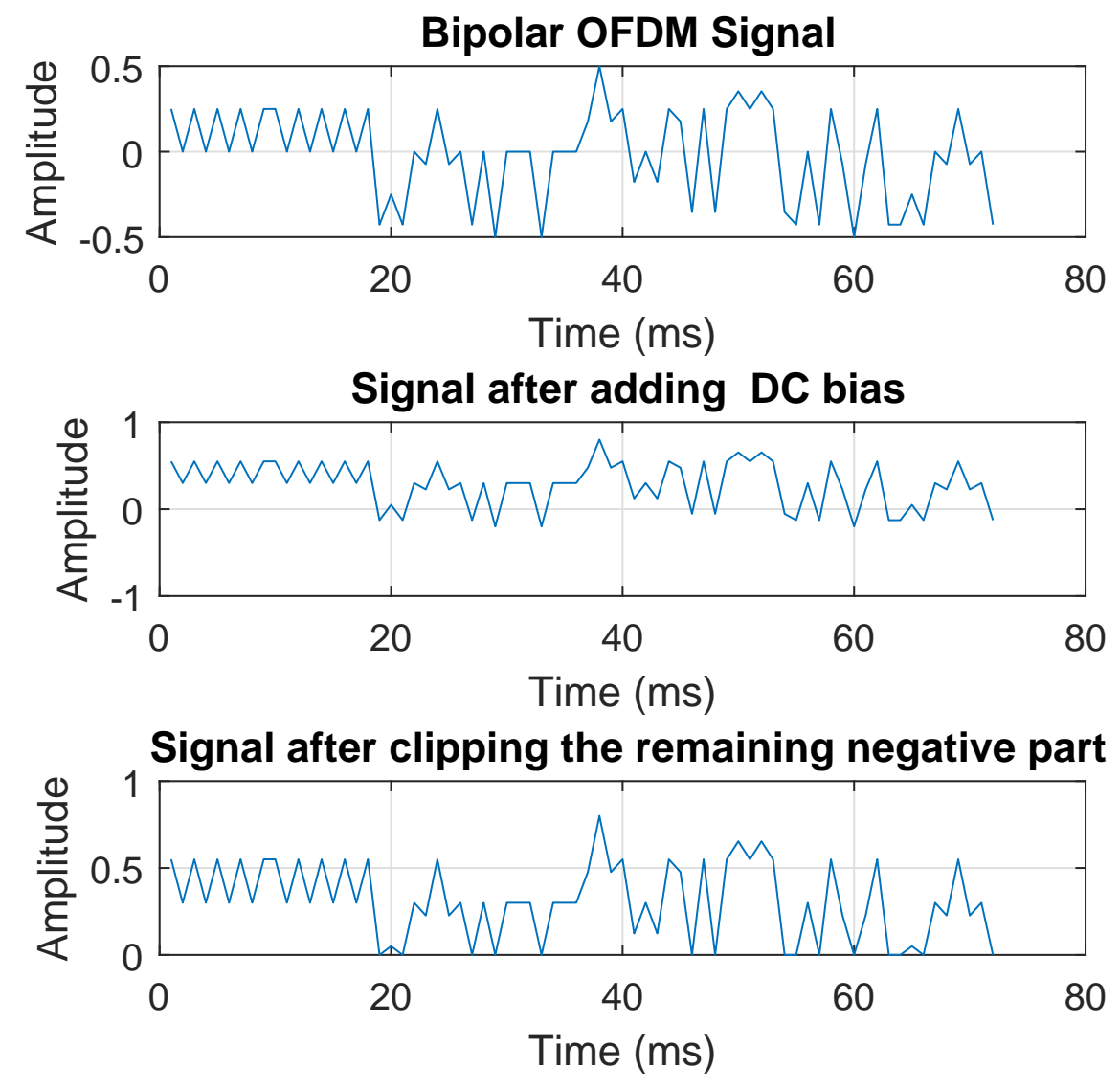

Figure 3.2: (a) OFDM bipolar signal (b) The signal after adding DC bias voltage (c) The signal after clipping the remaining negative.

bias value is added to this signal to convert it to unipolar and is plotted in Figure. 3.2 (b). Afterward, any remaining negative values are clipped at zero and is plotted in Figure. 3.2 (c), which introduces distortion noise. The added DC value increases the transmitter power requirement, while the clipping introduces clipping noise in both the even and odd subcarriers.

The transmitted signal $s(t)$ can be expressed as

$$
s(t)=x(t)+b_{d c}-e_{c}(t)
$$

where $b_{d c}$ is the bias voltage, and $e_{c}(t)$ is clipping noise with a variance $\sigma_{c_{D C O}}^{2}$. 
To minimize the clipping noise, the bias voltage $b_{d c}$ is given by [71]

$$
b_{d c}=2 \sigma_{x} \zeta
$$

where $\zeta$ is the biasing ratio and is given by [71]

$$
\zeta=\frac{b_{d c}}{c_{u}-c_{l}}=\frac{-c_{l}}{c_{u}-c_{l}}
$$

where $c_{u}$ and $c_{l}$ denote the upper and the lower clipping level respectively.

The variance of the clipping noise $\sigma_{c_{D C O}}^{2}$ is given by [72]

$$
\begin{aligned}
\sigma_{c_{D C O}}^{2}=\sigma_{x}^{2}+\left(b_{d c}^{2}-\sigma_{x}^{2}\right) Q\left(\frac{b_{d c}}{\sigma_{x}}\right)-b_{d c} \sigma_{x} g\left(\frac{b_{d c}}{\sigma_{x}}\right) & \\
& -\sigma_{x}^{2} Q\left(\frac{-b_{d c}}{\sigma_{x}}\right)^{2}-\left(\sigma_{x} g\left(\frac{b_{d c}}{\sigma_{x}}\right)-b_{d c} Q\left(\frac{b_{d c}}{\sigma_{x}}\right)\right)^{2}
\end{aligned}
$$

where $Q($.$) is the tail probability of the standard Gaussian distribution, g($.$) is the PDF of$ the clipping noise which follows Gaussian distribution and is given by [20]

$$
g(u)=\frac{1}{\sqrt{2 \pi \sigma_{x}^{2}}} e^{-\frac{\left(u-\frac{\zeta}{2} \sigma_{x}\right)^{2}}{2 \sigma_{x}^{2}}}
$$

The average transmitted optical power is expressed as

$$
E[s(t)]=\sigma_{x}\left(\frac{\zeta}{2}+\frac{1}{\sqrt{2 \pi}} e^{\frac{-\zeta^{2}}{8}}-\frac{\zeta}{2} Q\left(\frac{\zeta}{2}\right)\right)
$$

For fixed modulation scheme, the maximum channel capacity (bps/Hz) of the DCO-OFDM-VLC system can be given as

$$
R_{D C O}=\frac{1}{N+N_{c p}} \sum_{k=1}^{N / 2-1} \log _{2}\left(1+\frac{|H(k)|^{2} \sigma_{k}^{2}}{\sigma_{n}^{2}+|H(k)|^{2} \sigma_{c_{D C O}}^{2}+\sigma_{I S I}^{2}}\right)
$$

where $|H(k)|$ indicates the $k^{t h}$ channel transfer function, $N_{c p}$ indicates the number of cycling 
prefix of the OFDM, $\sigma_{n}^{2}$ indicates the ambient noise power which is given by (1.7), and $\sigma_{I S I}^{2}$ is ISI noise and is given by

$$
\sigma_{I S I}^{2}=\gamma^{2}\left(\sum_{k=1}^{N} \int_{T_{d}}^{\infty} h_{k}(t) \otimes s(t) d(t)\right)^{2}
$$

where $T_{d}$ denotes the transmitted optical pulse duration, and $k$ is channel index.

The maximum channel capacity is subject to (optical power constraint) $0 \leq E[s(t)] \leq P_{t}$, where $P_{t}$ is the transmitted power.

\subsubsection{ACO-OFDM Modulation Scheme}

In the ACO-OFDM technique, the modulated data is assigned to the odd indexed subcarriers, while the even indexed subcarriers are set to zero. Also, signals in this scheme can be transmitted without any DC-bias.

Referring to Figure. 3.1, the input vector $\mathbf{x}$ of the IFFT block can be given as

$$
\mathrm{x}=\left[\begin{array}{lllllllllllll}
0 & \mathrm{~s}_{0} & 0 & \mathrm{~s}_{1} & \ldots & \mathrm{s}_{\mathrm{N} / 4-1} & 0 & \mathrm{~s}_{\mathrm{N} / 4-1}^{*} & \ldots & 0 & \mathrm{~s}_{1}^{*} & 0 & \mathrm{~s}_{0}^{*}
\end{array}\right]
$$

After performing IFFT, the signal can be written as

$$
x\left[n+\frac{N}{2}\right]=-x[n] \quad n=0,1, \ldots, \frac{N}{2}-1
$$

Also, $x[n]$ can be considered a Gaussian random variable with zero mean and variance is given by

$$
\sigma_{x}^{2}=\frac{2}{N^{2}} \sum_{k=1}^{N / 4} \sigma_{2 k-1}^{2}
$$

In the ACO-OFDM scheme, bipolar to unipolar conversion is achieved by clipping the 
negative amplitudes which will not introduce any distortion to the transmission. LED turn on voltage $V_{\text {tov }}$, is added to shift the unipolar signal to the dynamic range of the LED. However, any amplitude levels exceeding the upper limit of the LED dynamic range $V_{\max }$ must be clipped, thus resulting into clipping noise.

The transmitted signal in an ACO-OFDM is written as

$$
s(t)= \begin{cases}x(t) & x(t)>0 \\ 0 & x(t) \leq 0\end{cases}
$$

where $s(t)$ is a clipped Gaussian signal.

The PDF of $s(t)$ is written as [73]

$$
f_{s(t)}(u)= \begin{cases}0.5 & u=0 \\ \frac{1}{\sqrt{2 \pi \sigma_{x}^{2}}} e^{\frac{-u^{2}}{2 \sigma_{x}^{2}}} & u>0\end{cases}
$$

The average power of $s(t)$ can be calculated as

$$
\begin{aligned}
E[s(t)] & =0.5 \times 0+\int_{0}^{\infty} u \frac{1}{\sqrt{2 \pi \sigma_{x}^{2}}} e^{\frac{-u^{2}}{2 \sigma_{x}^{2}}} d u \\
& =\frac{\sigma_{x}}{\sqrt{2 \pi}}
\end{aligned}
$$

The channel capacity of fixed modulation scheme for the ACO-OFDM is given by

$$
R_{A C O}=\frac{1}{N+N_{c p}} \sum_{k=1}^{N / 4} \log _{2}\left(1+\frac{|H(k)|^{2} \sigma_{2 k-1}^{2}}{4 \sigma_{n}^{2}+|H(k)|^{2} \sigma_{c_{A C O}}^{2}+\sigma_{I S I}^{2}}\right)
$$

where $\sigma_{c_{A C O}}^{2}$ is the variance of the clipping noise and is given by [20] 


$$
\sigma_{c_{A C O}}^{2}=\frac{1}{2} \sigma_{x}^{2} Q(\sqrt{2} \eta)\left(1+\eta^{2}\right)-\frac{\sigma_{x}^{2} \eta}{2 \sqrt{\pi}} e^{-\eta^{2}}
$$

where $\left(\eta=\frac{I_{\max }-I_{\text {bais }}}{\sigma_{x}}\right)$ is a dimming level, $I_{\max }$ and $I_{\text {bais }}$ are the maximum input current and bias current of the LED, respectively.

Equation (3.19) shows that, the spectral efficiency of ACO-OFDM is reduced by half as compared to DCO-OFDM, since only the odd subcarriers are used in transmission.

In this scheme, also, the maximum channel capacity is subject to (optical power constraint) $0 \leq$ $E[s(t)] \leq P_{t}$

\subsection{Performance Analysis and Bit Loading Algorithm}

In this section, we present a performance analysis of the V2V-VLC system using bit loading algorithm. In this model, we use DCO-OFDM and ACO-OFDM schemes for both LOS and NLOS signal paths. APD at receiver convert optical signal to electrical signal with an addition noise. The parameters values of APD can be found in Table. 1.2 and in [21].

\subsubsection{SNR Estimation of Subcarriers Using SVD}

The matched filter $\mathrm{p}(\mathrm{t})$ is applied to the received signal $y_{r}(t)$ and then its output is sampled at the rate of $T_{s}, y_{r}[n]=y\left(n T_{s}\right)$. The discrete signal can be written as

$$
\left.y_{r}[n]=\sum_{s=1}^{2}\left(\gamma\left(s_{s}[n]+e_{c}[n]\right) \otimes h_{r s}[n]\right)+n_{r}[n]\right)
$$

where $s, r$ are the transmitter and the receiver respectively with values $(1,2)$.

The discrete signal in frequency domain can be written as 


$$
Y_{r}(k)=\sum_{s=1}^{2}\left(\gamma H_{r s}(k)\left(S_{s}(k)+E_{c}(k)\right)+N_{r}(k)\right)
$$

The estimated channel matrix of $k^{\text {th }}$ subcarrier can be expressed as

$$
\mathbf{H}(\mathbf{k})=\left[\begin{array}{ll}
H_{11}(k) & H_{12}(k) \\
H_{21}(k) & H_{22}(k)
\end{array}\right]
$$

\subsubsection{SVD Based Precoding Design}

As RF and VLC have completely different system setups, the optimal structure of the user precoding matrix is also different. SVD based structure is optimal for RF communication systems [74], but is not optimal for VLC communication, especially when a large number of LEDs are used with a requirement of a large biasing voltage. Since we have assumed a small number of LEDs with small bias requirements in this work, SVD technique can be employed to calculate the gain of each sub-carrier. Also, this structure is practical to use in many respects; it can distribute the MIMO channel into a set of parallel non-interference channels [75]. Moreover, SVD-precoder scheme solves the channel correlation problem which causes low spatial multiplexing gain in visible light MIMO communication.

For SVD-precoder, the precoding matrix is given as

$$
\mathbf{H}=\mathrm{UD} \mathrm{V}^{*}
$$

where $*$ denotes Hermitian transpose, $\mathbf{V}$ is $2 \times 2$ unitary matrix, used in the transmitter to pre-process transmitted signal, $\mathbf{U}$ is an $2 \times 2$ is the receive combining unitary matrix, and $\mathbf{D}$ is $2 \times 2$ diagonal matrix with nonnegative diagonal elements (singular values of matrix $\mathbf{H})$. 
Therefore, the channels are decomposed into two independent parallel subchannels. The equivalent channel gain of the two independent subchannels is given as

$$
\mathbf{G}_{\mathbf{o}}=\left[\begin{array}{cccc}
\lambda_{11} & \lambda_{12} & \ldots & \lambda_{1 k} \\
\lambda_{21} & \lambda_{22} & \ldots & \lambda_{2 k}
\end{array}\right]
$$

The gain of each subcarrier can be found from the matrix $\mathbf{G}_{\mathbf{o}}$. Therefore, $S N R[k]$ of $k^{\text {th }}$ subcarrier can be calculated as

$$
S N R[k]=\frac{\lambda_{k}}{\sigma_{n}^{2}(k)}
$$

By considering a targeted BER (BER[k]) of the $k^{t h}$ subcarrier, the modulation order $M_{k}$ can be found using (3.27) [9].

$$
B E R[k]=\frac{\left(\sqrt{M_{k}}-1\right)}{\sqrt{M_{k}} \log _{2}\left(\sqrt{M_{k}}\right)} \operatorname{erfc}\left(\sqrt{\frac{3 S N R[k]}{2\left(M_{k}-1\right)}}\right)
$$

\subsubsection{Adaptive Transmission and Bit Loading Scheme}

Bit-loading is performed with respect to the frequency-selective channel and specific modulation order to improve the system's performance. Therefore, subcarriers having high attenuation are re-modulated with lower modulation orders. Similarly, the subcarrier having low attenuation are re-modulated with higher orders. The subcarriers of the O-OFDM signal are modulated by $M-Q A M$; the constellation order $M$ can differ from other subcarriers on the basis of SNR. We use Table. 3.2 to assign the bits for each subcarrier.

The number of bits per symbol of each subcarrier is between 1 and 8 . By obtaining $M_{k}$ for

each subcarrier, the transmission rate (bps) for the DCO-OFDM-VLC can be calculated as 


$$
R_{D C O_{\text {Adapive }}}=\frac{1}{\left(N+N_{c p}\right) T_{s}} \sum_{k=1}^{N / 2-1} \log _{2} M_{k}
$$

For the ACO-OFDM-VLC, the transmission rate can be given as

$$
R_{A C O_{\text {Adapive }}}=\frac{1}{\left(N+N_{c p}\right) T_{s}} \sum_{k=1}^{N / 4-1} \log _{2} M_{k}
$$

\subsection{Results and Discussions}

\subsubsection{Simulation Environment}

MATLAB software is used to model the V2V-VLC system, and parameters/values used in simulations are summarized in Tables. 1.2, 2.1, and 3.1. Multi-path propagation with one reflection system is considered assuming that all the reflectors surfaces have same reflective factor. Moreover, summer season with clear sky is considered. Also, vehicles are moving at same speed in the same direction. The city of Toronto is considered for sunlight intensity calculations, whose location coordinates are given in Table. 3.1. We do Monte Carlo simulation for 5,000 iterations for simulating the V2V-VLC channel. To reduce the impact of the channel causing signal distortion, truncated sinc pulse filter is used for pulse shaping. Also, We use Philips Ultinon LED 12985BWX2 as transmitter [59]. Moreover, Hamamatsu-S8664 APD is considered as a receiver [21]. The scheme proposed in [76] is used to model the nonlinearity of LEDs I-V property. Figure. 3.3 shows that, simulated I-V property fits well with the data obtained from the LED datasheet. Moreover, the linear I-V curve is obtained by linear regression of the data. 


\subsubsection{Results and Analysis}

The system performance is analyzed by considering various parameters for both types of the O-OFDM schemes. The distributions of bit- and power-loading of the DCO-OFDM for 64 subcarriers at luminance intensity $(1000-2500(c d))$ with an $8 \mathrm{~dB}$ and $10 \mathrm{~dB}$ bias is shown in Figure. 3.4. 128 QAM (7 bits/symbol) is assigned to subcarriers having an SNR $\geq 14 d B$, Binary Phase Shift Keying (BPSK) (1 bit/symbol) is chosen for the subcarriers having SNR $=7 d B$ (as seen with $8 \mathrm{~dB}$ bias) and no bits assigned for SNR $<7 d B$. The transmitter can obtain the modulation order $M_{k}$ to be employed for each subcarrier by using Table. 3.2. Also, Figure. 3.4 shows, clipping noise is decreased with the increase in bias. Therefore, for $10 \mathrm{~dB}$ bias, as the clipping noise is less, the total noise power will depend more on channel frequency response rather on $8 \mathrm{~dB}$ bias. As a result of $8 \mathrm{~dB}$ bias, the bit allocation

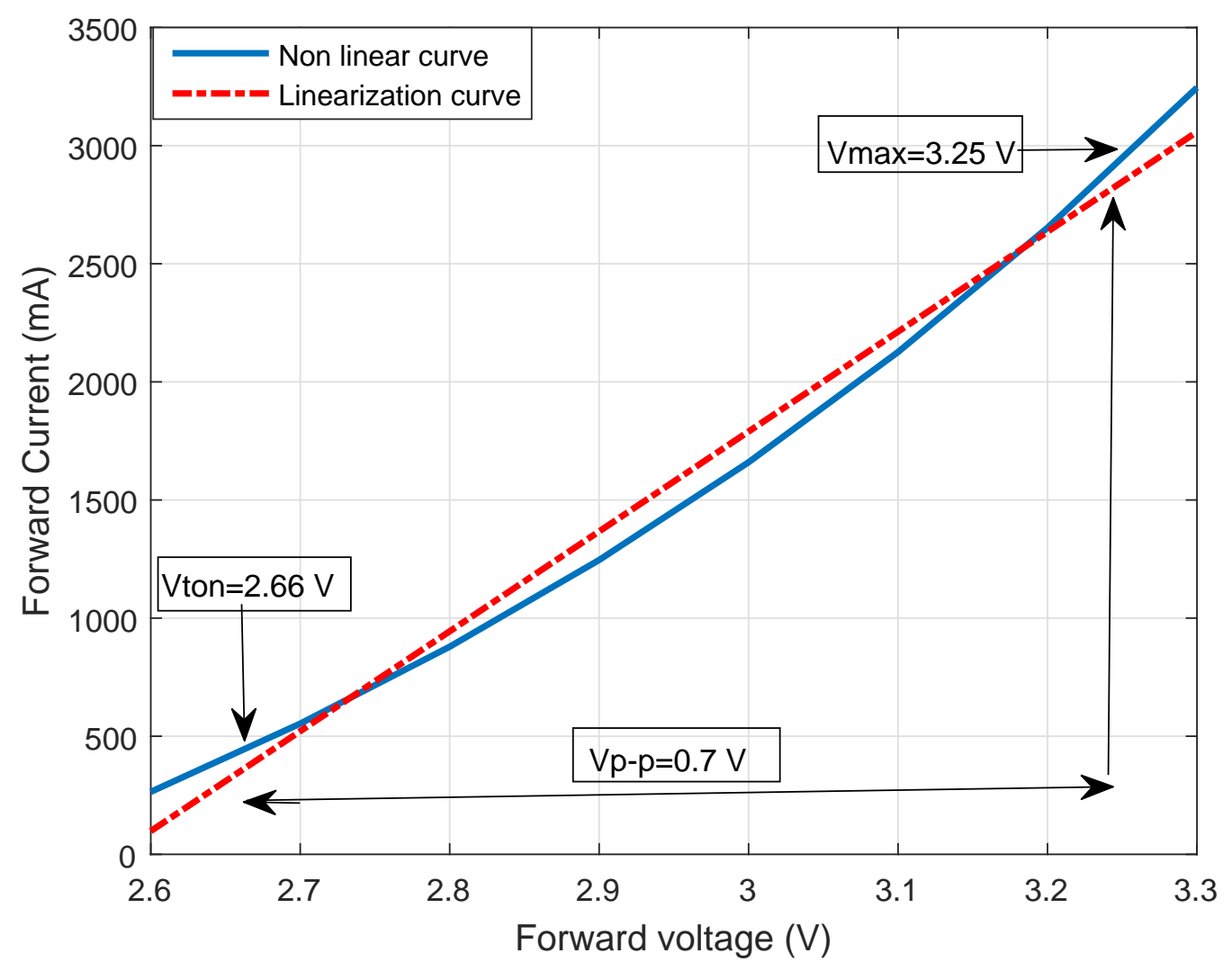

Figure 3.3: I-V characteristic curve of the LED. 
Table 3.1: Simulation parameters

\begin{tabular}{|c|c|}
\hline \hline The Parameter & Value \\
\hline Toronto location & $43.6532 N, 79.3832 \mathrm{~W}$ \\
\hline Weather & Clear Sky \\
\hline Number of FFT points & 64 \\
\hline Subcarrier modulation scheme & 4-QAM, 16-QAM,...256-QAM \\
\hline Signal bandwidth & $50 \mathrm{MHz}$ \\
\hline Cyclic prefix length $C P$ & 8 \\
\hline Targeted BER & $10^{-5}$ \\
\hline Biased voltage (V) & $8 \mathrm{~dB}, 10 \mathrm{~dB}$ \\
\hline Vehicles speed (same direction) & $30 \mathrm{Km} / \mathrm{h}$ \\
\hline
\end{tabular}

decreases by two bits over 64 subcarriers. Moreover, Figure. 3.4 shows the power distribution and modulation order of the subcarriers has decreasing trends at high frequency spectrum. Reason for this type of behavior depends upon the low-pass property of the LED. In Figure. 3.5, bit loading and power distribution for different subcarriers for the ACO- OFDM adaptive modulation scheme is presented, under similar channel conditions as in the DCO-OFDM with an $8 \mathrm{~dB}$ bias and $10 \mathrm{~dB}$ bias respectively. In this technique, only the odd subcarriers are used for data transmission. Also, the power distribution and modulation order of the subcarriers are decreasing with the high frequency spectrum. Moreover, a maximum of 256 QAM (8 bits/symbol) is assigned to subcarriers having an SNR of about $12 d B$, whereas the BPSK (1 bit/symbol) format is chosen for the subcarriers having SNR $=3 d B$, and no bits assigned for $\mathrm{SNR}<3 d B$. Note that, the number of assigned bits to the ACO-OFDM scheme is higher than the DCO-OFDM scheme. This is because the DCO-OFDM experiences higher clipping distortion compared to ACO-OFDM at certain illuminance intensity. Therefore, the number of loaded bits per subcarrier for the DCO-OFDM is generally lower than in the ACO-OFDM. Also, Figure. 3.5 shows that increasing the bias decreases the clipping noise. As a result of an $8 \mathrm{~dB}$ bias, the bit allocation decreases by one bit over 32 subcarriers. 
Table 3.2: Look-Up-Table

\begin{tabular}{|c|c|c|}
\hline \hline \multicolumn{2}{|c|}{ Number of bits /Subcarrier } \\
\hline$M_{k}$ & DCO-OFDM-SNR (dB) & ACO-OFDM-SNR (dB) \\
\hline 0 & $<7$ & $<3$ \\
\hline 1 & 7 & 3 \\
\hline 2 & 8 & 4 \\
\hline 3 & 9 & 5 \\
\hline 4 & 10 & 6 \\
\hline 5 & 11 & 7 \\
\hline 6 & 12 & 8 \\
\hline 7 & $>=14$ & $>=12$ \\
\hline 8 & NA & $>$ \\
\hline
\end{tabular}
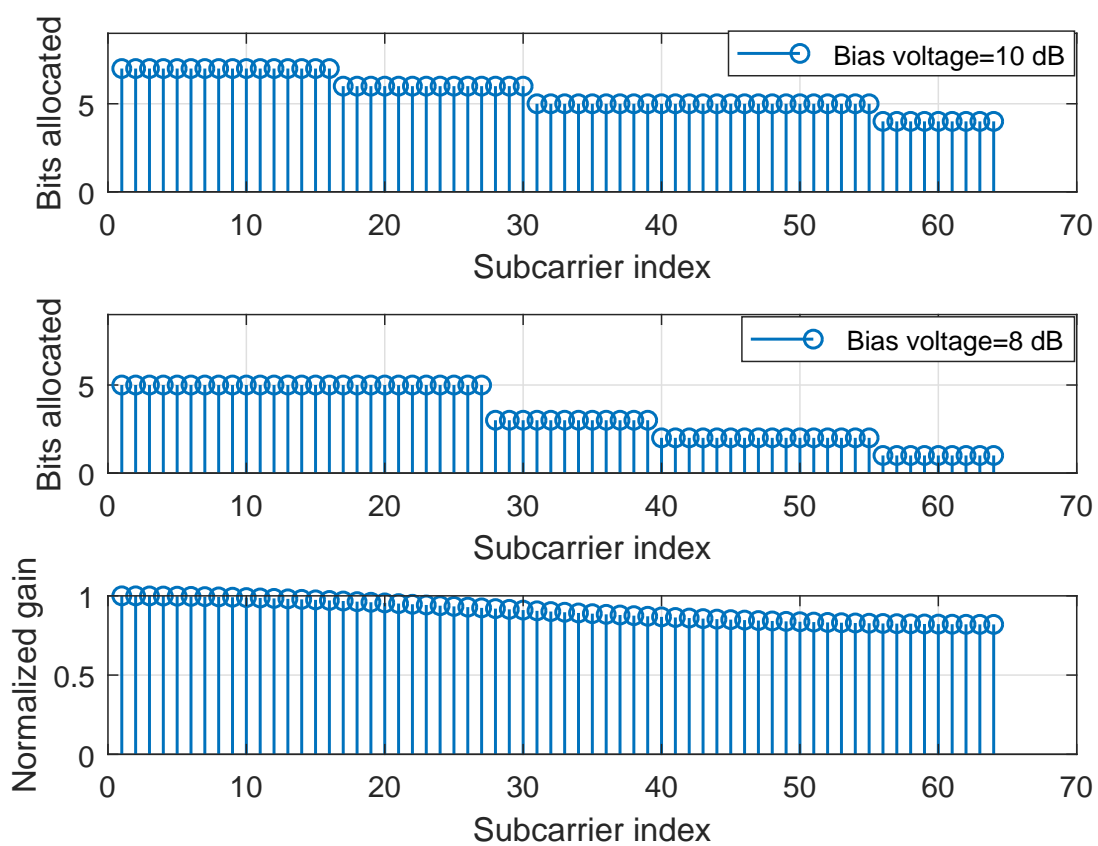

Figure 3.4: Number of loaded bits for DCO-OFDM when $I_{T}\left(\alpha_{T}, \beta_{T}\right)=1500(c d)$.

\subsubsection{Transmission Rate Analysis}

In Figure. 3.6, and Figure. 3.7, transmission rates for adaptive DCO-OFDM and ACO-OFDM, are presented. The adaptive transmissions are compared with the fixed modulation schemes while maintaining the desired BER of $10^{-5}$. In Figure. 3.6, it is shown that by employing adaptive transmission in the DCO-OFDM, data rate is significantly improved, compared 

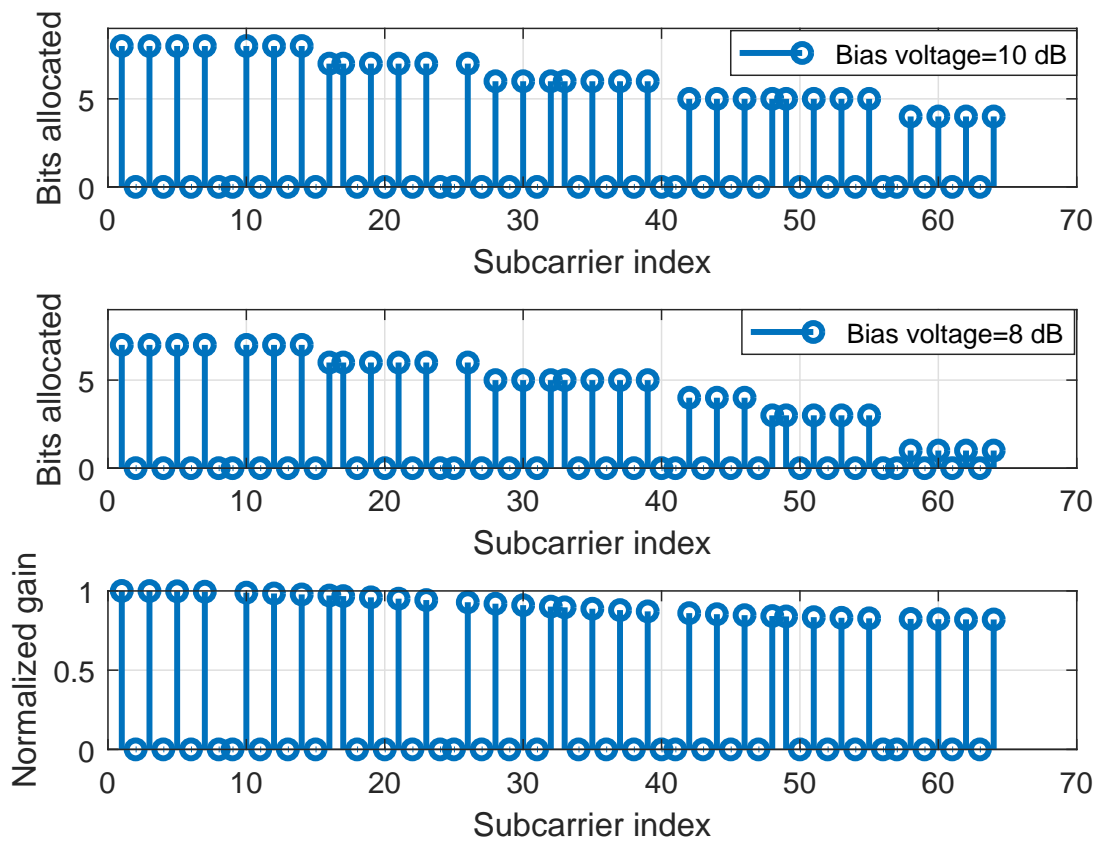

Figure 3.5: Number of loaded bits for ACO-OFDM when $I_{T}\left(\alpha_{T}, \beta_{T}\right)=1500(c d)$.

to fixed modulation. Furthermore, the clipping distortion affects the performance of the DCO-OFDM, above luminance intensity of $1600(c d)$. In Figure. 3.7, the transmission rate for the ACO-OFDM is calculated and it is observed that the clipping distortion degrades the performance when the luminance intensity exceeds $2000(c d)$. Moreover, the maximum achievable rate for the ACO-OFDM is lower than the DCO-OFDM for both types of transmissions schemes;i,e. adaptive and fixed. As the ACO-OFDM utilizes a dynamic range more effectively due to half the number of data subcarriers.

\subsubsection{BER Analysis}

BER performance for both, the ACO-OFDM and the DCO-OFDM is investigated, for adaptive and fixed modulation schemes. Figure. 3.8 and Figure. 3.9, show better SNR performance for adaptive modulation than fixed modulation, for both schemes. Moreover, it can be seen that adaptive modulation schemes can meet the target of BER at less SNR value than other QAM schemes. Also, the BER performance is not recorded for SNR range of (0 - 


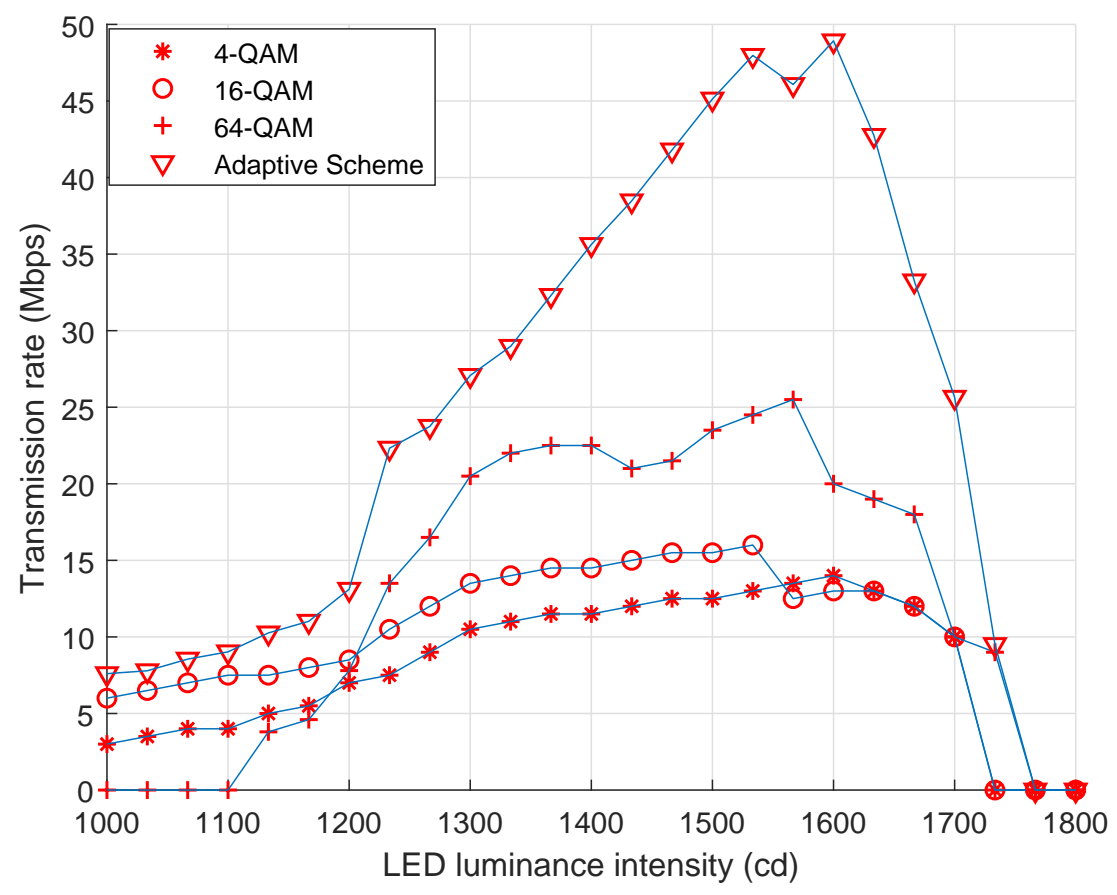

Figure 3.6: The transmission rate of DCO-OFDM with adaptive modulation.

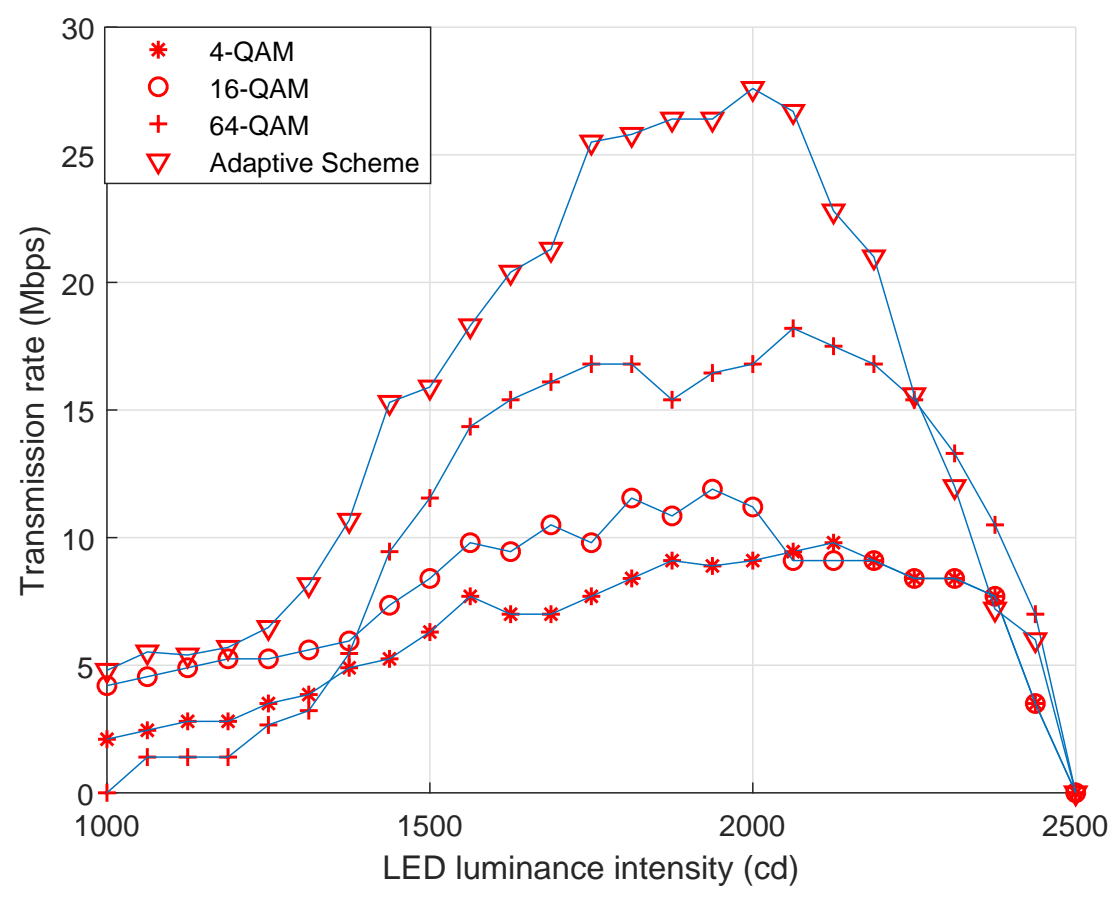

Figure 3.7: The transmission rate of ACO-OFDM with adaptive modulation. 


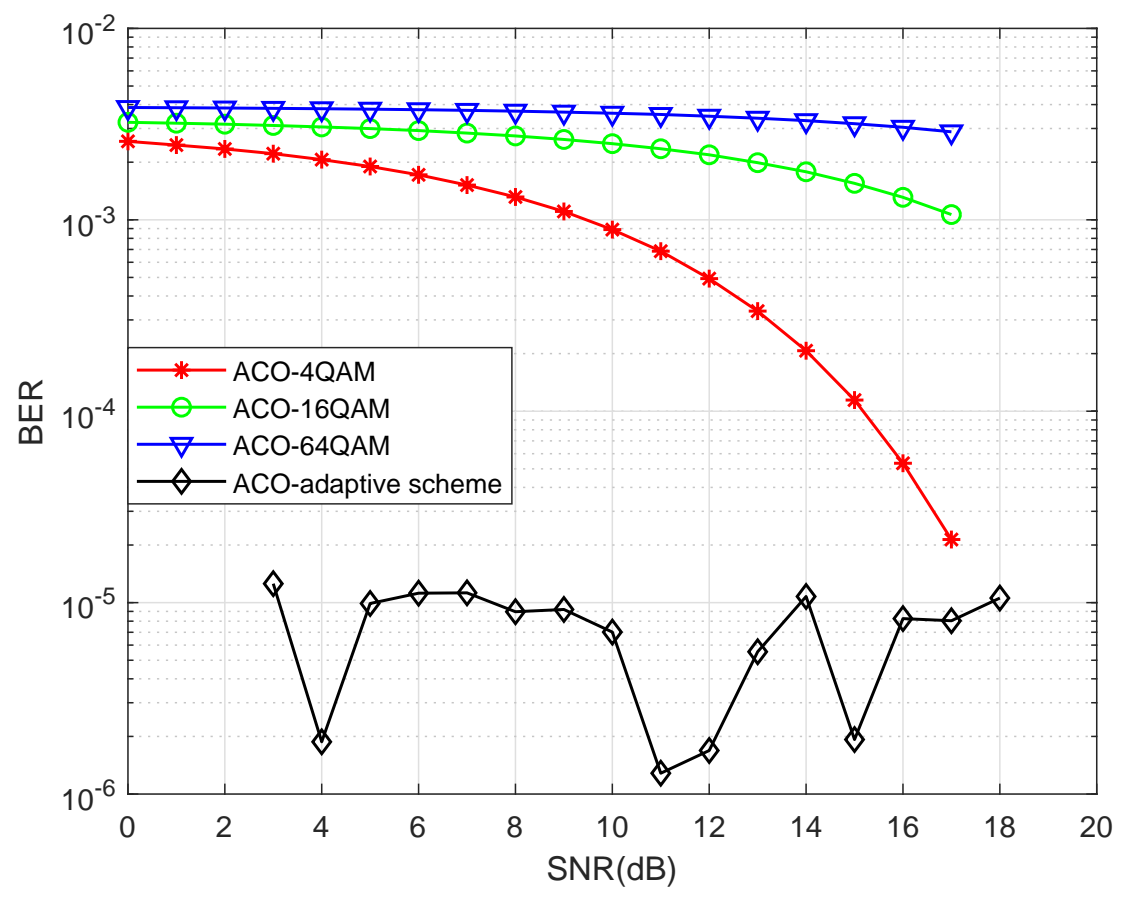

Figure 3.8: SNR vs. BER for ACO-OFDM with FFT size=64.

$3 \mathrm{~dB}$ ) for the ACO-OFDM, and for SNR range of (0 - $7 \mathrm{~dB})$ for the DCO-OFDM because no bits are transmitted at all in these ranges ( according to the bit loading algorithm). Moreover, the BER performance is not recorded for SNR $>18 d B$ for both schemes because the clipping distortion degrades the performance. Although, Figure. 3.8 and Figure. 3.9 show a better performance of the ACO-OFDM comparing with the DCO-OFDM. This happens due to the inherent characteristics of the DCO-OFDM, being sensitive to clipping noise. 


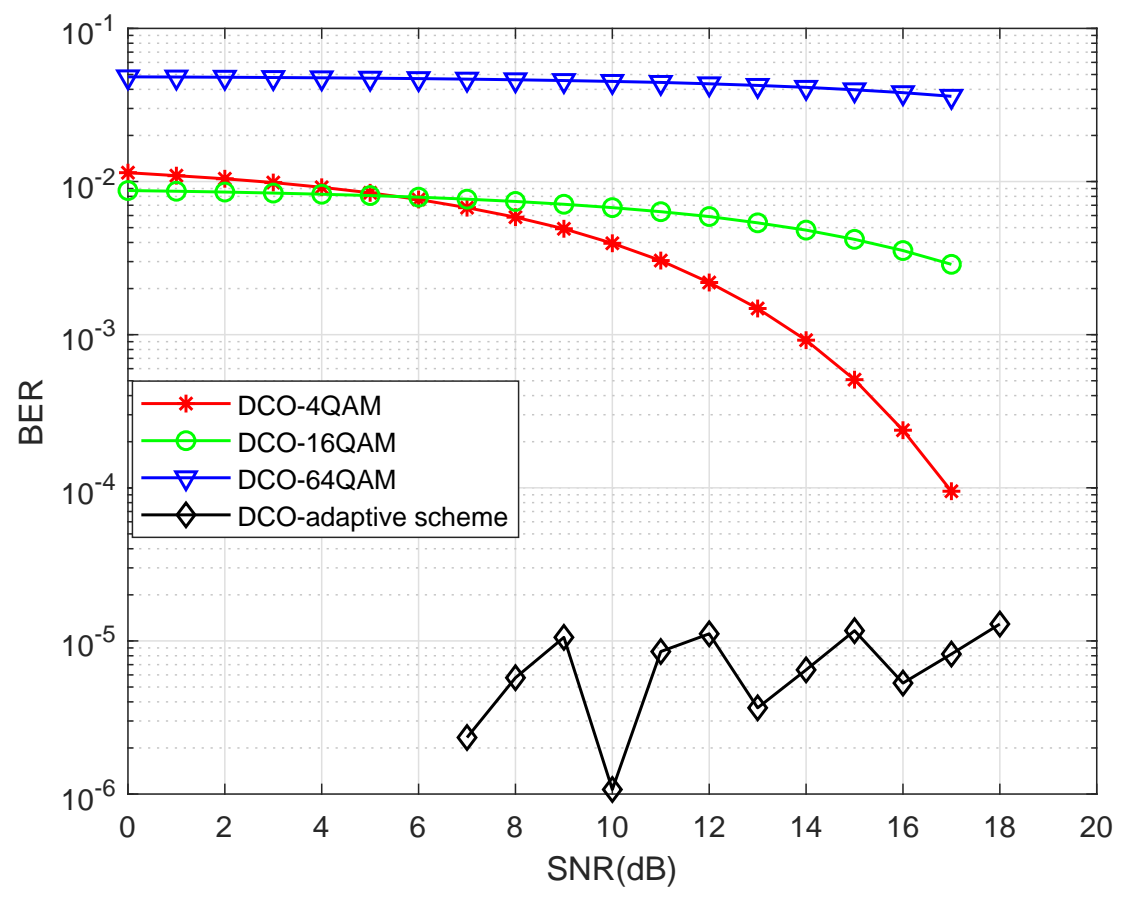

Figure 3.9: SNR vs. BER for DCO-OFDM with FFT size $=64$.

\subsection{Chapter Summary}

This chapter investigates and analyzes adaptive modulation schemes for O-OFDM based V2V-VLC system considering the practical noise environment to fully exploit the transmission resources. Two modulation schemes are used, DCO-OFDM and ACO-OFDM to combat ISI and to improve the data rate. We compare the performance of the adaptive modulation schemes with fixed modulation schemes in terms of BER, and data rate, where the adaptive schemes show superiority in performance. 


\section{Chapter 4}

\section{Proposed Precoder and Equalizer in $2 \times 2$ MIMO VLC Systems for Vehicular Application}

Precoding is a technique which exploits transmit diversity by weighting the information stream, i.e. the transmitter sends the coded information to the receiver to achieve pre-knowledge of the channel. VLC channels inherently exhibit frequency-selective and multi-path fading effects that result in ISI and thus reduces the data rate. To this end, optical MIMO can provide spatial diversity and thus achieve a high data rate. Inspired by these facts, we investigate different precoding and equalization schemes for $2 \times 2 \mathrm{MIMO}$ for the V2V-VLC system. In this work, we consider three detection schemes, ZF, ML, and MMSE. Also, we consider flickering/dimming control and the nature of outdoor VLC channel as a frequency selective channel. Moreover, we investigate a transceiver considering perfect Channel State Information (CSI) based on different detection algorithms. Simulation results of the investigated transceiver demonstrate improvements in BER and Mean Squared Error (MSE) for the MMSE scheme. 


\subsection{Related Work in Precoding and Equalization for VLC}

There is limited published work in precoding and equalization areas in MIMO-V2V-VLC System. Most of the existing work on the precoding and equalization of VLC systems are mainly limited to indoor environments. Tomas et al. [77] designed transceiver model for realistic simulation of vehicular VLC. They tested the design of VLC transceiver prototype using a modified version of 802.11 MAC protocol, showing that a MAC protocol specifically

designed for VLC should take into account its specific physical properties. In [78], the authors studied a linear transceiver design for indoor VLC with multiple LEDs, they showed that for MIMO-VLC, the optimal precoding reduces to a simple LED selection strategy. Corresponding channel structure and simple receiver design for VLC systems were also discussed in [79]. The MIMO transceiver was optimized to improve the system performance in [80]. The power and the positive offset are jointly designed to improve the spectral efficiency by taking BER requirement, nonnegativity constraint and sum optical power constraint of the transmit signals. The authors in [81] investigated a joint precoding matrix and receiving matrix design via a convergence guaranteed iterative algorithm by considering the positive constraint on the transmit signals into account. In another work [81], Ying et al. investigated the design of optical wireless MIMO precoder and equalizer systems for indoor VLC. The authors studied the system with perfect channel state and imperfect channel state information. In [82], a multi-user downlink channel was considered and the corresponding precoding design was optimized. The authors imposed the ZF structure on the precoding matrix. In [83], power and offset allocation with adaptive modulation were well discussed in SVD-based MIMO systems for optical wireless channels, but dimming control was not supported. Authors in [84] proposed a modified SVD-VLC MIMO system to meet the illumination constraints, but additional adjusting should be applied to the input signals so that both the nonnegativity of the intensity modulation and dimming control can be 


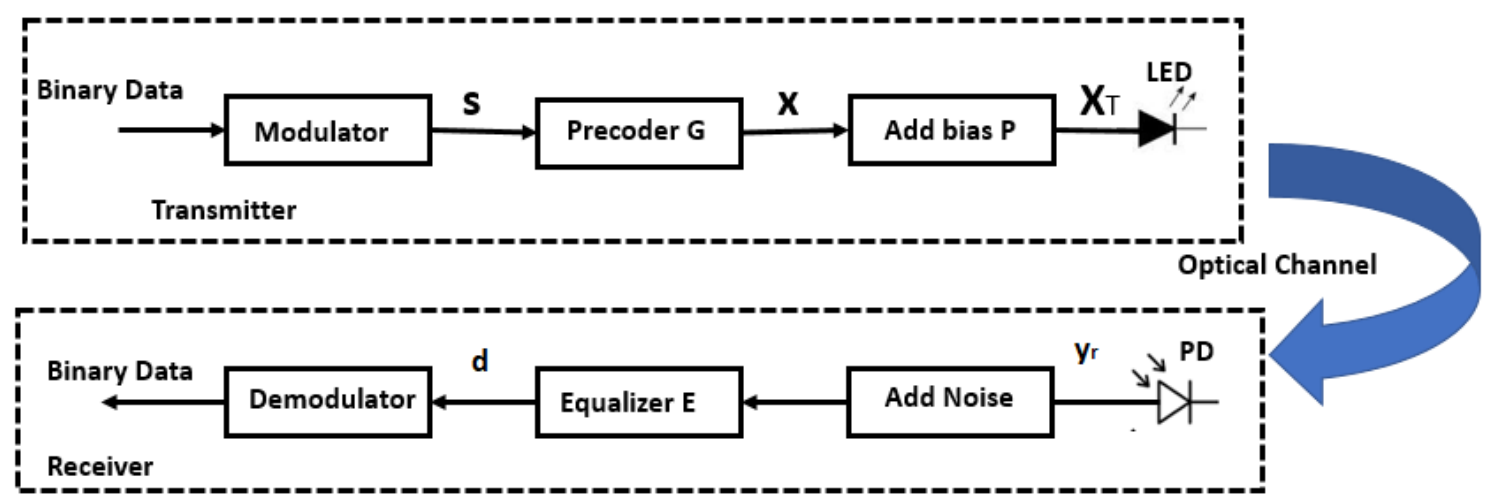

Figure 4.1: Illustration of the investigated precoder and equalizer system.

satisfied. In the area of MIMO VLC system, the precoder and equalizer design are still open to researchers especially in outdoor-VLC applications.

\subsection{System Model}

Considering the system given in section 2.2 and shown in Figure. 2.1, the precoder-equalizer system for the V2V-VLC system is shown in Figure. 4.1, where the binary data modulated, then precoded by the precoder $\mathbf{G}$, then DC-biased is added to ensure that the transmitted signal is positive. To this end, the signal is transmitted through an optical channel. In the receiver, the received signal is equalized by equalizer $\mathbf{E}$, then passes to be demodulated to predict the transmitted data.

\subsubsection{Investigating the Transmitter}

Since we have $2 \times 2$ MIMO systems, the original information bits are modulated into source data vector denoted by

$$
\mathbf{s}(t)=\left[\begin{array}{ll}
s_{1}(t) & s_{2}(t)
\end{array}\right]^{T}
$$

where any bit $s_{i}$ assumed to be Return to Zero (RZ) with zero mean and two levels $[-A, A]$. The source data vector will be multiplied by an $2 \times 2$ precoding matrix $\mathbf{G}$, where $\mathbf{G}$ is given 


$$
\mathbf{G}=\left[\begin{array}{ll}
g_{11} & g_{12} \\
g_{21} & g_{22}
\end{array}\right]
$$

Each entry of $\mathbf{G}$ denotes the precoding weight of the transmitted signal between the transmitter $s$ and the receiver $r,(r, s=1,2)$.

The precoded data vector $\mathbf{x}$ is given by

$$
\mathbf{x}=\mathrm{G} \mathbf{s}
$$

It is worth noting that since VLC is based on intensity modulation, the vector $\mathbf{x}$ should be positive. Thus, a DC biasing vector $\mathbf{p}=\left[\begin{array}{ll}p_{1} & p_{2}\end{array}\right]^{T}$ is added to the transmitted signal to ensure that the transmitted signal is positive which guarantees that each transmitted vector $\left(\mathbf{x}_{\mathbf{T}_{1}}, \mathbf{x}_{\mathbf{T}_{\mathbf{2}}}\right)$ is positive.

$$
\mathbf{x}_{\mathbf{T}}=\mathbf{G} \mathbf{s}+\mathbf{p}
$$

The transmitted data vector $\mathbf{x}_{\mathbf{T}}$ is calculated for the VLC system as follows

$$
\left[\begin{array}{l}
x_{T_{1}} \\
x_{T_{2}}
\end{array}\right]=\left[\begin{array}{ll}
g_{11} & g_{12} \\
g_{21} & g_{22}
\end{array}\right]\left[\begin{array}{l}
s_{1} \\
s_{2}
\end{array}\right]+\left[\begin{array}{l}
p_{1} \\
p_{2}
\end{array}\right]
$$

Let $l$ and $u$ be the lower and the upper levels of the LED dynamic range respectively. For each LED, we assume that $[l, u](0<l)$. It is the dynamic-range constraint, and in order to meet the dimming control, i.e., to achieve the illumination requirement by controlling the output power, the following condition must be fulfilled, $l \leq x_{T_{1}}, x_{T_{2}} \leq u$. 
Thus, the mean value of any transmitted vector is given by

$$
E\left[x_{T_{s}}\right]=\sum_{s=1}^{2} \sum_{r=1}^{2} g_{r s} E\left[s_{s}\right]+E\left[p_{s}\right]=p_{s}
$$

Equation (4.6) shows that the transmitted signal average is positive and it thus ensures that the transmitted signal is positive, $E\left[s_{s}\right]=0$ is the mean value of the transmitted bit, $E\left[p_{s}\right]$ is the mean value of the added bias voltage, and $s$ indicates the transmitter and $r$ indicates the receiver with values 1 or 2 .

To keep the transmitted signal within the LED dynamic range, the following two constraints should be satisfied $\sum_{s=1}^{2} \sum_{r=1}^{2}\left|g_{r s}\right| A \leq u-p$, and $\sum_{s=1}^{2} \sum_{r=1}^{2}\left|g_{r s}\right| A \leq p-l$.

To meet the brightness control of the vehicle's headlights

$$
a b s(\mathbf{G}) A \leq \min (u-p, p-l)
$$

The DC bias vector can affect the performance of precoder and equalizer. The BER

performance is best when the DC bias is the midpoint of $l$ and $u$. That is, $p=\frac{1}{2}(l+u)$, we can get a high-performance precoder and equalizer under the constraint of (4.7) [81].

\subsubsection{Investigating the Receiver}

At the receiver, PDs generate an electric signal proportional to the intensity of the received optical signals. Then, the received signal vector can be expressed as follows

$$
\mathbf{y}_{\mathbf{r}}=\gamma \mathbf{H} \mathbf{x}_{\mathbf{T}}+\mathbf{n}
$$

where $\mathbf{y}_{\mathbf{r}}$ is the received vectors, $\mathbf{H}$ indicates the channel matrix comprises of LOS and NLOS components and $\mathbf{n}$ is the total noise vector. Here, we consider an Additive White Gaussian Noise (AWGN) following the distribution of $N\left(0, \sigma^{2} I\right)$. 
For $2 \times 2$ MIMO, the channel matrix $\mathbf{H}$ is given by

$$
\mathbf{H}=\left[\begin{array}{ll}
h_{11} & h_{12} \\
h_{21} & h_{22}
\end{array}\right]
$$

Since we have LOS path and NLOS path coming by the reflectors within the test area, each entry of matrix $\mathbf{H}$ can be given as

$$
h(t)=h_{L O S}(t)+h_{N L O S}(t)
$$

Considering a two dimensional (2-D) system, i.e all sources and receivers lie in the same plane, each entry of LOS path in $\mathbf{H}$ is given by

$$
h_{r s}=\frac{\cos \left(\phi_{r s}\right) \cos \left(\theta_{r s}\right) A_{r} T_{s}\left(\theta_{r s}\right) g\left(\theta_{r s}\right)}{\pi R_{r s}^{2}} .
$$

where $\phi_{r s}$ denotes the emitting angle between source $s$ and receiver $r, \theta_{r s}$ denotes the incident angle between source $s$ and receiver $r, A_{r}$ denotes the effective area of the receiver, $T_{s}\left(\theta_{r s}\right)$ denotes the signal transmission coefficient of an optical filter, and $g\left(\theta_{r s}\right)$ denotes the concentrator gain.

Any entry in the channel matrix $\mathbf{H}$ for NLOS path between transmitter $s$ and receiver $r$ through reflector $q$ can be calculated as follows

$$
h_{r s(N L O S)}=h_{q s}+h_{r q}
$$

It is to be noted that the NLOS gain factor denoted by $h_{r s}(N L O S)$ consists of two components, the gain between the transmitter $s$ and reflector $q$, denoted by $h_{q s}$, and the gain between the reflector $q$ and the receiver $r$, denoted by $h_{r q}$. These gains are calculated as follows 


$$
h_{q s}=\frac{\cos \left(\phi_{q s}\right) \cos \left(\theta_{q s}\right) A_{q}}{\pi R_{q s}^{2}}
$$

where $\phi_{q s}$ denotes the emitting angle between source $s$ and reflector surface $q, \theta_{q s}$ denotes the incident angle between source $s$ and reflector surface $q, R_{q s}$ denotes the distance between source $s$ and reflector surface $q, A_{q}$ denotes the effective area of the reflector surface $q$.

The gain factor $h_{r q}$ is given by

$$
h_{r q}=\rho \frac{\cos \left(\phi_{r q}\right) \cos \left(\theta_{r q}\right) A_{r} T_{r}\left(\theta_{r q}\right) g\left(\theta_{r q}\right)}{\pi R_{r q}^{2}} .
$$

In the above equation, $\phi_{r q}$ is the emitting angle between reflector $q$ and receiver $r, \theta_{r q}$ is the incident angle between receiver $r$ and reflector surface $q, R_{r q}$ denotes the distance between receiver $r$ and reflector surface $q$, and $\rho$ is the reflectivity of the reflector surface.

Equation (4.8) can be expanded to two received signals, one from the LOS, and another one from NLOS, $\mathbf{y}_{\mathbf{r}}=\mathbf{y}_{\mathbf{r}_{\mathbf{L O S}}}+\mathbf{y}_{\mathbf{r}_{\mathrm{NLOS}}}$, where $\mathbf{H}=\mathbf{H}_{L O S}+\mathbf{H}_{N L O S}$.

The received signal which is given by (4.8) can be written as

$$
\mathbf{y}_{\mathbf{r}}=\gamma \mathbf{H}(\mathbf{G} \mathbf{s}+\mathbf{p})+\mathbf{n}
$$

At the receiver side, the term $\mathbf{H p}$ is subtracted from $\mathbf{y}_{\mathbf{r}}$ before the equalization. After this subtraction, the communication model can be written as

$$
\hat{\mathbf{y}_{\mathbf{r}}}=\gamma \mathbf{H G s}+\mathbf{n}
$$

The estimated symbols by the equalizer can be expressed as follows 


$$
\begin{aligned}
\hat{\mathbf{s}} & =\mathbf{E}\left(\mathbf{y}_{\mathbf{r}}-\mathbf{H P}\right) \\
& =\mathbf{E}(\gamma \mathbf{H G} \mathbf{s}+\mathbf{n})
\end{aligned}
$$

\subsubsection{Equalization Matrix Design}

An equalizer is a digital filter that is used to mitigate the effects of intersymbol interference that is introduced by a time-dispersive channel. In order to recover the source data from the received signal, we can formulate the problem as follows "design a precoder matrix $\mathbf{G}$ at the transmitter and an equalizer matrix $\mathbf{E}$ at the receiver to minimize the MSE between the transmitted data and the recovered data", that is,

$$
\begin{array}{ll}
\underset{\mathbf{G}, \mathbf{E}}{\operatorname{MSE}} & (\mathbf{d}, \mathbf{s}, \mathbf{G}, \mathbf{E}) \\
\text { subject to } & a b s(\mathbf{G}) A \leq \min (u-p, p-l)
\end{array}
$$

where $\mathbf{d}=\gamma \mathbf{E} \mathbf{H} \mathbf{G} \mathbf{s}+\mathbf{E} \mathbf{n}$.

Here, we assume that the channel matrix $\mathbf{H}$ is perfectly known. Also, we assume the data vector $\mathbf{s}_{\mathbf{s}}$ is taken from one of a M-Pulse Amplitude Modulation (PAM) symbol with $M=2^{k}$ and $k$ is the number of bits per symbol.

\subsubsection{Zero Forcing Equalizer (ZF)}

The ZF equalizer is a linear equalization algorithm used in communication systems, which inverts the frequency response of the channel. This algorithm applies the inverse of the channel to the received signal, to restore the signal before the channel. In this method, a linear time-invariant filter is used and the ISI component at the output of the equalizer is forced to zero. This will be useful when ISI is significant compared to noise.

The ZF equalization matrix is given by [85] 


$$
\mathbf{E}_{Z F}=\left(\mathbf{H}^{\mathbf{H}} \mathbf{H}\right)^{-\mathbf{1}} \mathbf{H}^{\mathbf{H}}
$$

The $\mathbf{E}_{\mathbf{Z F}}$ matrix should have non-zero off diagonal elements and hence this cancel out the interference signal. In fact, it is simple to implement and easy to analyze. The main disadvantage of this equalizer is amplifying the noise. Using ZF equalization, the estimated transmitted symbol vector is [85]

$$
\begin{aligned}
\hat{\mathbf{s}} & =\gamma \mathbf{E}_{\mathbf{Z F}} \mathbf{y}_{\mathbf{r}} \\
& =\gamma\left(\mathbf{H}^{\dagger} \mathbf{H} \mathbf{G} \mathbf{s}+\mathbf{H}^{\dagger} \mathbf{n}\right) \\
& =\gamma\left(\mathbf{G} \mathbf{s}+\mathbf{H}^{\dagger} \mathbf{n}\right)
\end{aligned}
$$

where $(.)^{\dagger}$ means the Pseudo inverse of a matrix.

\subsubsection{Maximum Likelihood Equalizer (ML)}

ML detection calculates the Euclidean distance between the received signal vector and the product of all possible transmitted signal vectors with the given channel $\mathbf{H}$, and finds the one with the minimum distance. ML detection determines the estimate of the transmitted signal vector. The maximum of the likelihood function is achieved when $\left|\mathbf{y}_{\mathbf{r}}-\mathbf{H} \hat{\mathbf{s}}\right|^{2}$ reaches the minimum value, therefore the estimation of ML detector is given by [86]

$$
\hat{\mathbf{S}} \hat{\mathbf{M} L}=\arg \min _{\mathbf{s}_{\hat{\mathrm{M} L} \in \mathbf{S}^{\mathrm{M}}}}\left\|\mathbf{y}_{\mathbf{r}}-\mathbf{H} \hat{\mathbf{s}}\right\|^{2}
$$

\subsubsection{Minimum Mean Squared Error Equalizer (MMSE)}

For MMSE equalizer we have [75] 


$$
\begin{aligned}
& M S E(\mathbf{d}, \mathbf{s}, \mathbf{G}, \mathbf{E}) \\
& =E\left[\|\mathbf{d}-\mathbf{s}\|^{\mathbf{2}}\right] \\
& =E\left[\|(\mathbf{E H G}-\mathbf{I}) \mathbf{s}+\mathbf{E n}\|^{\mathbf{2}}\right] \\
& =\operatorname{Tr}\left((\mathbf{E H G}-\mathbf{I}) \mathbf{D}(\mathbf{E H G}-\mathbf{I})^{\mathbf{H}}\right)+\operatorname{Tr}\left(\mathbf{E R}_{\mathbf{n}} \mathbf{E}^{\mathbf{H}}\right) \\
& \left.=\operatorname{Tr}\left((\mathbf{E H G D})(\mathbf{E H G})^{\mathbf{H}}\right)\right)+\operatorname{Tr}\left(\mathbf{E} \mathbf{R}_{\mathbf{n}} \mathbf{E}^{\mathbf{H}}\right)+\operatorname{Tr}(\mathbf{D})-\operatorname{Tr}(\mathbf{E H G D})-\operatorname{Tr}\left(\mathbf{D}(\mathbf{E H G})^{\mathbf{H}}\right)
\end{aligned}
$$

where $\mathbf{D}=E\left[\mathbf{s ~ s}^{\mathbf{H}}\right], \mathbf{R}_{\mathbf{n}}=\mathbf{E}\left[\mathbf{n n}^{\mathbf{H}}\right]=\sigma_{\mathbf{n}}^{\mathbf{2}} \mathbf{I}_{\mathbf{2}}$, and $\mathbf{D}=\operatorname{diag}[D, D]$, where $D$ is given by

$$
D=\frac{A^{2}(M+1)}{3(M-1)}
$$

Here, we assume the data vector $\mathbf{s}_{\mathbf{s}}$ is taken from one of a M-PAM symbol with $M=$ $2^{k}$ and $k$ is the number of bits per symbol.

The optimal $\mathbf{E}$ for given $\mathbf{G}$ satisfies the following condition

$$
\frac{d M S E(\mathbf{d}, \mathbf{s}, \mathbf{G}, \mathbf{E})}{d \mathbf{E}}=0
$$

Then the MMSE equalizer is given by

$$
\mathbf{E}=\mathbf{D ~ G}^{\mathbf{H}} \mathbf{H}^{\mathbf{H}}\left(\mathbf{H ~ G ~ D ~ G}{ }^{\mathbf{H}} \mathbf{H}^{\mathbf{H}}+\sigma_{\mathbf{n}}^{2} \mathbf{I}_{2}\right)^{-1}
$$

The related MSE covariance matrix can be given as

$$
\begin{aligned}
\Re & =E\left[(\mathbf{s}-\hat{\mathbf{s}})(\mathbf{s}-\hat{\mathbf{s}})^{\mathbf{T}}\right] \\
& =\left(\mathbf{D}^{-\mathbf{1}}+\frac{\mathbf{1}}{\sigma_{\mathbf{n}}^{2}} \mathbf{G}^{\mathbf{H}} \mathbf{H}^{\mathbf{H}} \mathbf{H} \mathbf{G}\right)^{-\mathbf{1}}
\end{aligned}
$$




\subsubsection{Precoding Matrix}

\subsubsection{MMSE Precoder}

Since MMSE equalization technique is investigated, here we will use the corresponding precoder matrix which is given by [87]

$$
\mathbf{G}_{M M S E}=\sqrt{\frac{1}{2}} \mathbf{V} \triangle \widetilde{\mathbf{D}}
$$

where $\mathbf{V}$ comes from the decomposition of channel matrix $\mathbf{H}^{*} \boldsymbol{\Phi}_{\mathbf{n n}}^{-\mathbf{1}} \mathbf{H}=\mathbf{V} \boldsymbol{\Theta} \mathbf{V}^{*}, \boldsymbol{\Phi}_{\mathbf{n n}}=$ $\mathbf{2} \sigma_{\mathbf{n}}^{\mathbf{2}} \mathbf{I}$ is the noise matrix, $\widetilde{\mathbf{D}}$ is a normalized discrete Fourier Transform matrix, and $\boldsymbol{\Delta}$ is a diagonal matrix with the diagonal elements determined by [88]

$$
\left\|m_{i i}\right\|=\frac{1}{2}\left(1+\sum_{j=1}^{2} \theta_{j}^{-1}\right)-\theta_{i}^{-1}
$$

where $\theta_{i}$ are diagonal elements of $\Theta$ coming from the eigenvalue decomposition $\mathbf{H}^{*} \mathbf{\Phi}_{\mathbf{n n}}^{-\mathbf{1}} \mathbf{H}=$ $\mathbf{V} \Theta \mathbf{V}^{*}$.

\subsubsection{ZF Precoder}

The precoder for ZF Equalization is [85]

$$
\mathrm{G}_{\mathrm{ZF}}=\sqrt{\frac{1}{2 \operatorname{tr}\left(\Theta^{-1 / 2}\right)}} \mathrm{V} \Theta^{-1 / 4} \widetilde{\mathrm{D}}
$$




\subsubsection{ML Precoder}

The precoder for ML Equalization is given by [86]

$$
\mathbf{G}_{\mathbf{M L}}=\left[\begin{array}{ll}
g_{m 11} & g_{m 12} \\
g_{m 21} & g_{m 22}
\end{array}\right]
$$

subject to the following constraints

$g_{m 11} g_{m 21}+g_{m 12} g_{m 22}=0$ and $g_{m 11}^{2}+g_{m 12}^{2}+g_{m 21}^{2}+g_{m 22}^{2}=0$.

\subsection{Simulation and Results}

We use MATLAB to model the system and simulation parameters are summarized in Tables. 1.2 and Table. 2.1. In the simulations, we consider multi-path propagation with one reflection system where all reflectors surfaces assume to have same reflective factor. Moreover, without loss of generality, we consider a clear sky and the vehicles are moving at uniform speed in the same direction. We use Philips Ultinon LED 12985BWX2 as transmitter [59]. Also, we use APD model (Si) Hamamatsu S8664 - 1010 as a receiver [21]. Moreover, we also consider 4-PAM modulated symbols and the constellation of 4-PAM is formed in the range of $[-3,3]$ (i.e. $A=3)$.

The investigated optimal precoder and equalizer can be easily applied to the practical design. We generate and fix the NLOS gain as given by (2.36). Both the signals and the noises are assumed to be independent, which is also reasonable in V2V-VLC practical systems. Additionally, we consider the ambient noise model used in [89]. To measure the performance of the different schemes, we compare the performance of the investigated precoders and equalizers in terms of BER and MSE. Notice that the power constraint in (4.7) is still valid for all the obtained simulations.

Figure. 4.2 shows the comparison of the investigated schemes from BER standpoint. The MMSE precoding scheme shows better improvement in terms of BER. For MMSE and ML 
detectors, we can see here that performance curve of these two systems are close to each other when SNR is low, but gap gets larger when SNR gets higher, while the performance of ZF shows a gap with ML and MMSE for all values of SNR. For high SNR the gap is about $2 \mathrm{~dB}$ between MMSE and ML, while the gap between ZF and the two other schemes is about $8 \mathrm{~dB}$. When SNR is less that means noise is large and the different detection schemes have almost same behavior. When the SNR is large, the post detection of SNR may have affected by channel matrix $\mathbf{H}$.

Figure. 4.3 shows a comparison of the suggested detection algorithms in terms of MSE. The MSE is a measure of the quality of an estimator, it is always non-negative as shown in Figure. 4.3. However, the MSE values for all detection schemes are very close and very low which mean those schemes can be valid to use with V2V-VLC system as the values closer to zero are better.

In general, MMSE shows the best performance than ML and ZF because MMSE detector dependent on the noise variance and the condition number of the channel matrix,

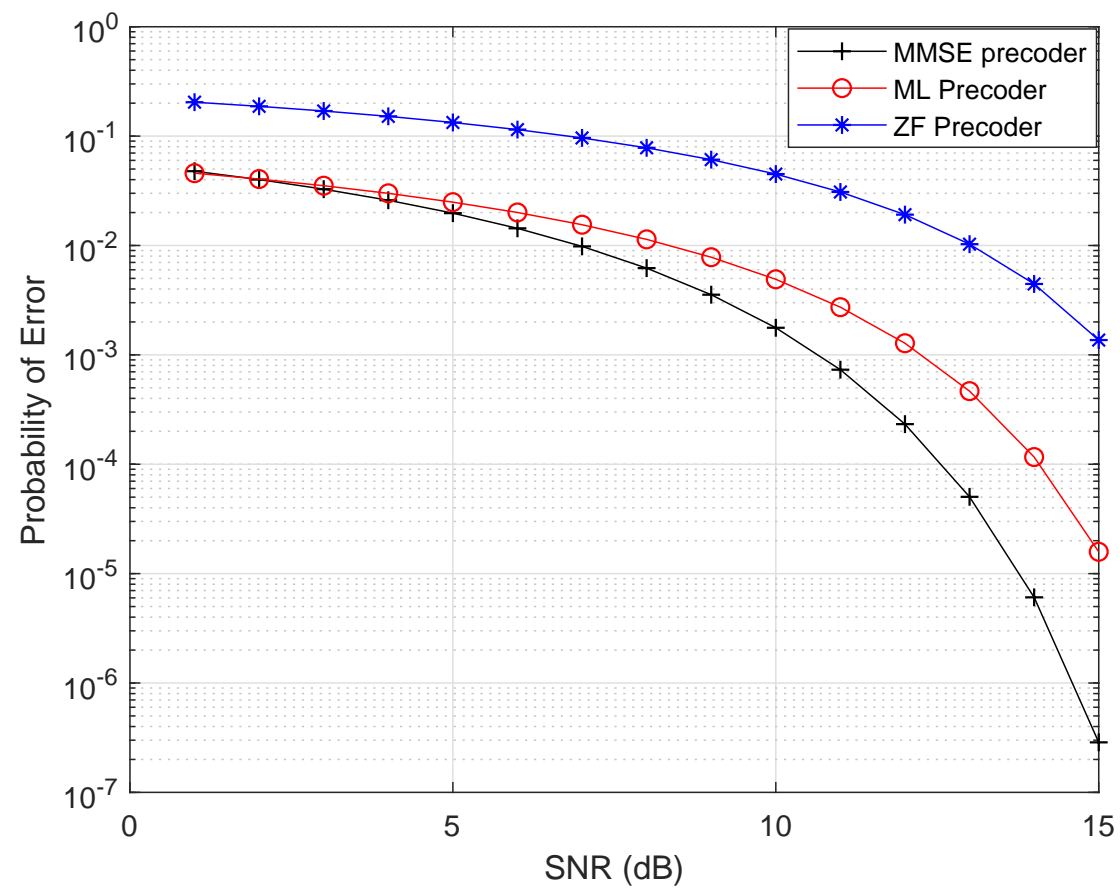

Figure 4.2: Comparison of the suggested detection algorithms in terms of BER. 


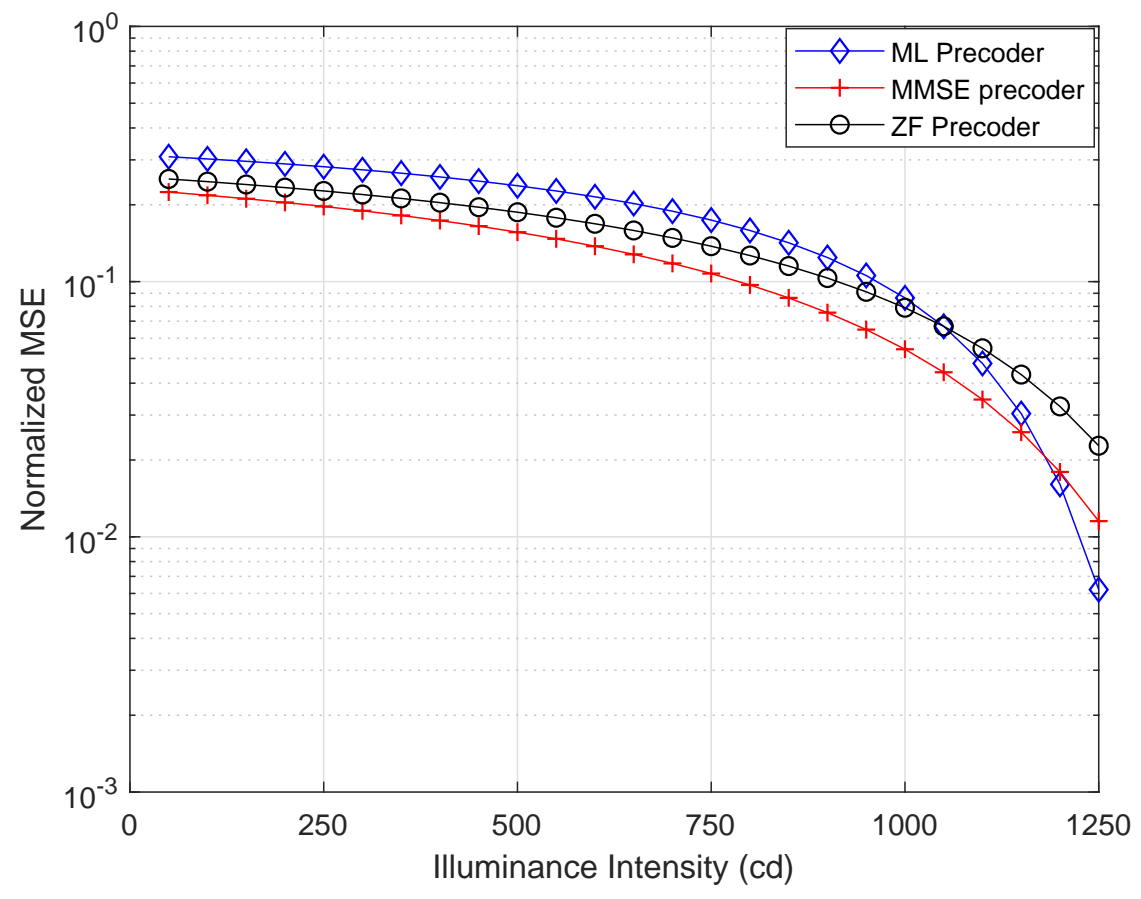

Figure 4.3: Comparison of the suggested detection algorithms in terms of MSE.

\subsection{Chapter Summary}

This chapter investigates the precoding and equalizing scheme for the V2V-VLC system considering flickering/dimming control and the nature of the outdoor VLC channel as a frequency selective channel to enhance the system's performance. We present the precoder matrix, as well as the equalizer matrix. We compare the system performance of MMSE, ZF, and ML precoders, where the MMSE scheme shows a noticeable improvement. 


\section{Chapter 5}

\section{Shadowing Effects on V2V-VLC}

Due to the nature of the visible light spectrum, shadowing is a significant issue especially over short distances and small FOVs. This can seriously impair communications in both quasi-stationary indoor and fast varying outdoor $\mathrm{V} 2 \mathrm{~V}$ environments.

However, by employing transmitters with a wide FOV as well as effectively utilizing the diffraction phenomenon of the light, the issues caused by shadowing can be alleviated. Note it is much better to implement diffuse transmitters which radiate the light over a wide solid angle, which does not have as severe pointing and shadowing problems when compared to point-to-point links. The diffused transmitter does not need to be aimed directly at the receiver since the radiated optical waves reflect from multiple surfaces.

In this chapter, we will discuss the effect of the shadowing on V2V-VLC. We proposed a method to take advantage of the optical diffraction phenomenon to overcome shadowing by employing a receiver with a wide FOV. We have modeled the shadowing effect for visible light by a bimodal distribution and derived the probability of error for no, moderate and severe shadowing conditions. The bimodal distribution effectively represents separate propagation modes caused by different polarizations. This approach is useful in both indoor and outdoor environments. 


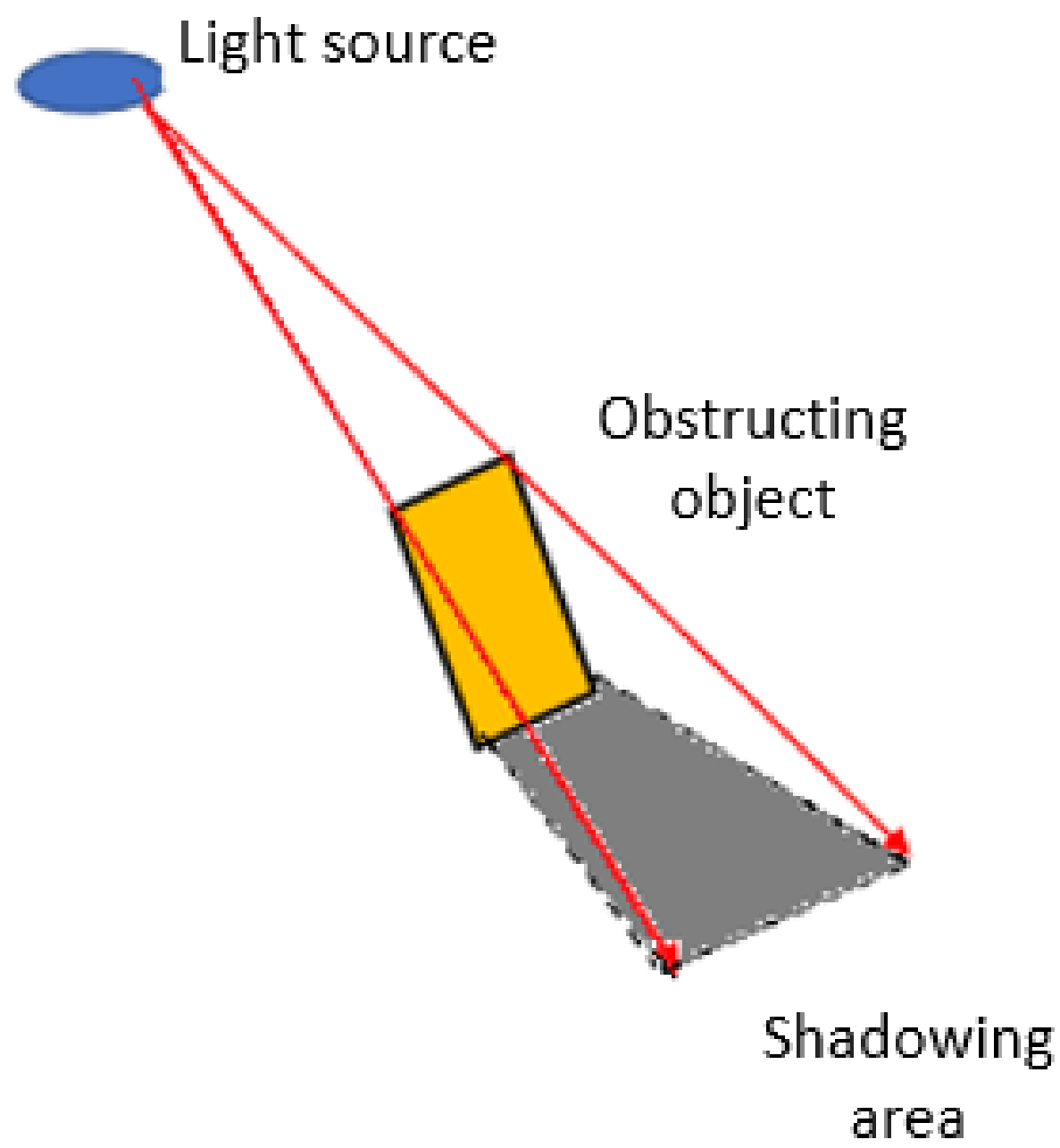

Figure 5.1: Outdoor shadowing

\subsection{Channel Impulse Response with Shadowing}

To mitigate the effect of shadowing, spatial diversity is commonly implemented where multiple transmitters and receivers are deployed around the shadowed area [79]. However, having such a setup is not always guaranteed and sustainable, henceforth it is necessary to study the effect of shadowing without having spatial diversity. This is necessary to effectively design a VLC system, along with obtaining the parameters that control its performance determined by the signal error probability under various shadowing conditions.

In this work, we are interested in the cases of no (absent), moderate and severe shadowing. 
In a typical VLC system, the channel impulse response for a bimodal obstruction is

$$
\begin{aligned}
& h_{1}(t)=\frac{\left(\frac{l+1}{2 \pi}\right) A_{r} \cos ^{l}(\phi) \cos (\theta)}{R_{1}{ }^{2}} \delta\left(t-\frac{R_{1}}{c}\right) \\
& h_{2}(t)=\frac{\left(\frac{l+1}{2 \pi}\right) A_{r} \cos ^{l}(\phi) \cos (\theta)}{R_{2}{ }^{2}} \delta\left(t-\frac{R_{2}}{c}\right)
\end{aligned}
$$

where $l$ denotes the order of the source, $R_{1}$ and $R_{2}$ are the total distance from the lighting source to the receiver, taking into account the light diffraction around the obstructing object from the first edge and the second edge of the obstructing object, respectively, $\phi$ is the emission angle, $\theta$ is the incident angle, and $c$ is the speed of the light.

The received signal energy $E_{r}$ is,

$$
E_{r}=\int_{0}^{T}\left|x(t) \otimes\left(h_{1}(t)+h_{2}(t)\right)\right|^{2} \mathrm{~d} t .
$$

where $x(t)$ denotes the transmitted signal, $T$ denotes the overall bit duration, and $\otimes$ means convolution sign.

\subsection{Shadowing Effect}

In a standard VLC system without shadowing, it can be assumed that the transmitted signal encounters AWGN, hence the distribution at the receiver remains Gaussian. Since shadowing plays a significant role in VLC, the final distribution will result in a multi-mode distribution depending on the severity of the shadowing effect as well as the complexity of the obstructing object (i.e. non-uniform objects with complex cavities cause secondary interference effects thereby varying the channel gain) but we will only consider a bimodal distribution here for 
simplicity.

As apparent in Figure. 5.1 (assuming the light only passes over two edges), we can assume a bimodal power distribution. With a bimodal approach, and assuming the PD receiver holds a large FOV, the optical power will be received from two distinct edges passing the object, each with different polarization.

Therefore, depending on the severity/complexity of the object surface, each polarizing path passing the object will appear as a local maximum.

Since we are considering the simple case of the light passing only from two sides of the object, this can be represented as a bimodal distribution with two peaks represent bit "1" and bit "0" respectively. The separation between the peaks of this distribution in a time-varying environment can be represented by two means and variances which both depend on time.

The distance between the means of the two normal functions which is roughly the distance between the two peaks is given by [90]

$$
S(t)=\frac{\mu_{1}(t)-\mu_{0}(t)}{2\left(\sigma_{0}(t)+\sigma_{1}(t)\right)}
$$

where $\mu_{0}, \sigma_{0}$, are the mean and the standard deviation of the first Gaussian distribution (bit "0"), $\mu_{1}, \sigma_{1}$ are the mean and the standard deviation of the second Gaussian distributions (bit "1"), and $\mu_{1}>\mu_{0}, S(t)$ is a bimodal separation at a certain time.

As shown in Figure. 5.2, the Bimodal distribution has the following PDF [91]

$$
\begin{aligned}
& f\left(z, \mu_{0}, \sigma_{0}, \mu_{1}, \sigma_{1}\right)= \frac{1}{2 \sqrt{2 \pi\left(\sigma_{0}\right)^{2}}} \exp \left(\frac{-\left(z-\mu_{0}\right)^{2}}{2\left(\sigma_{0}\right)^{2}}\right) \\
&+\frac{1}{2 \sqrt{2 \pi\left(\sigma_{1}\right)^{2}}} \exp \left(\frac{-\left(z-\mu_{1}\right)^{2}}{2\left(\sigma_{1}\right)^{2}}\right)
\end{aligned}
$$

Notice that when $S(t)$ decreases with a uniform ideal obstruction with minimal polarizing effects, the distribution will approach a standard Gaussian distribution and the received 


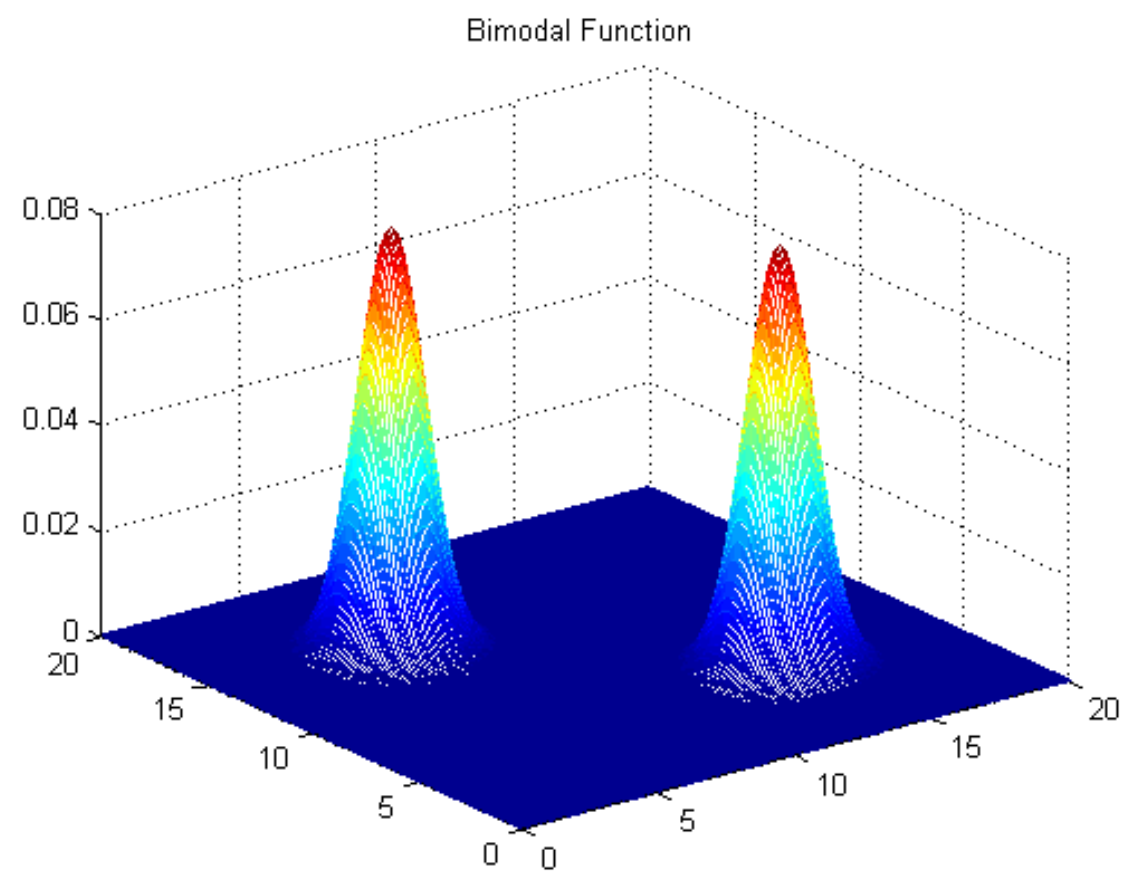

Figure 5.2: Bimodal function with equal variances

power will contain only a single maxima. 


\subsection{Error Probability}

In short-distance LOS links, a multipath dispersion is insignificant. Hence, LOS links channel are often only modeled with a linear attenuation and delay [92]. Optical LOS links are considered as non-frequency selective where the path loss depends on the square of the distance between the transmitter and the receiver.

Now, we will consider the error probability derivation with shadowing effect under a bimodal distribution case assuming OOK modulation is employed, also, we assume that a LEDs have a Lambertian radiation pattern [93]. This can, however, be extended to a multi-modal case.

To derive the probability of error, $P_{e}$ of the received signal for the three cases of shadowing: no-shadowing, moderate and severe shadowing, we will consider Figure. 5.3, Figure. 5.4, and Figure. 5.5 to illustrate their effect.

In this work, we present a closed formula for the probability of error for each case of shadowing, hence, we will show the derivation for the first case, while, the other cases can be derived using a similar procedure.

It is important to note that in the case of no shadowing, the transmitted light will be received without being significantly deteriorated by the shadowing object, thus, the received signal will be modeled as a Gaussian distribution for both cases of transmitting 1 and 0 represented by $s_{1}$ and $s_{0}$, with average values $\mu_{0}, \mu_{1}$ respectively as illustrated in Figures 5.3.

In the case of having moderate or severe shadowing, the light will be more affected by the shadowing object, hence the signal will be received from both sides which will result in a bimodal distribution. This is clearly depicted in Figure. 5.4, and Figure. 5.5, where two Gaussian distributions are created for each received bit, thereby resulting in a bimodal distribution.

The average expected received values can be represented by four vertical lines, with two possible average values for the case of receiving $s_{1}$, represented with average values $\mu_{1}, \mu_{1}^{\prime}$ and two possible average values for the case of receiving $s_{0}$ represented by $\mu_{0}, \mu_{0}^{\prime}$. 


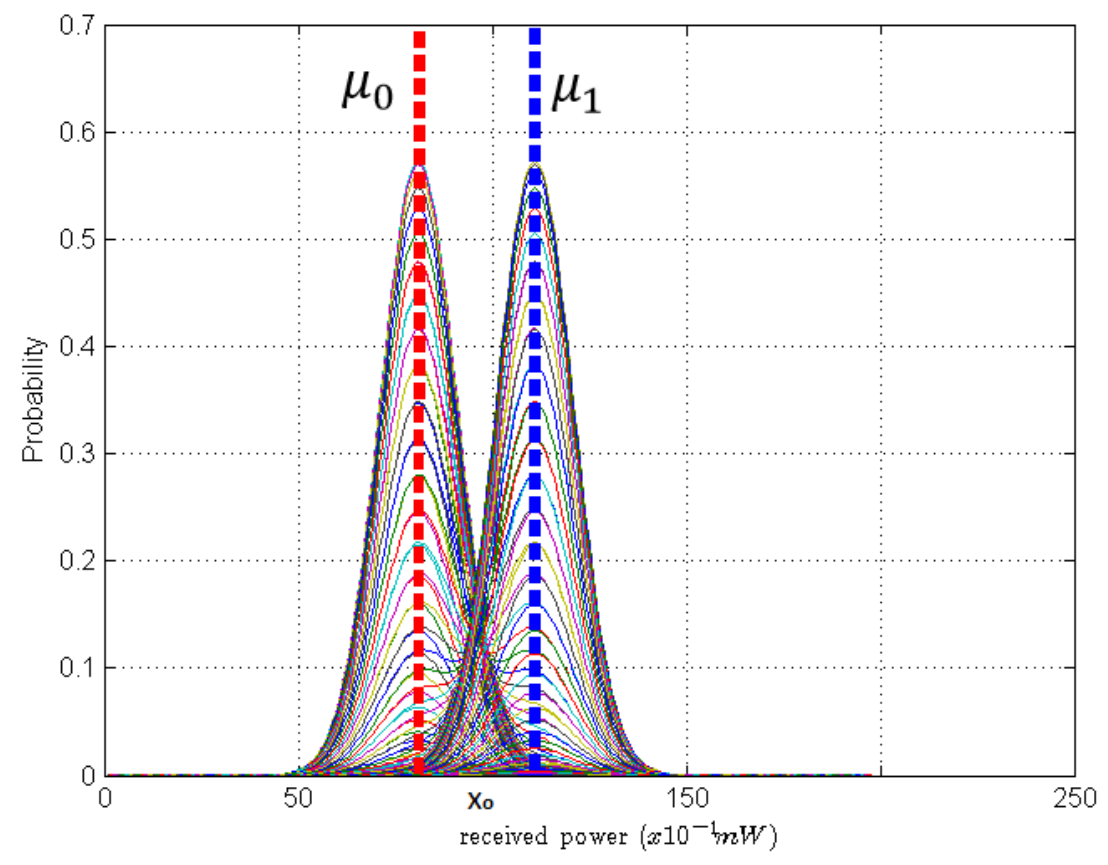

Figure 5.3: The PDF of no shadowing

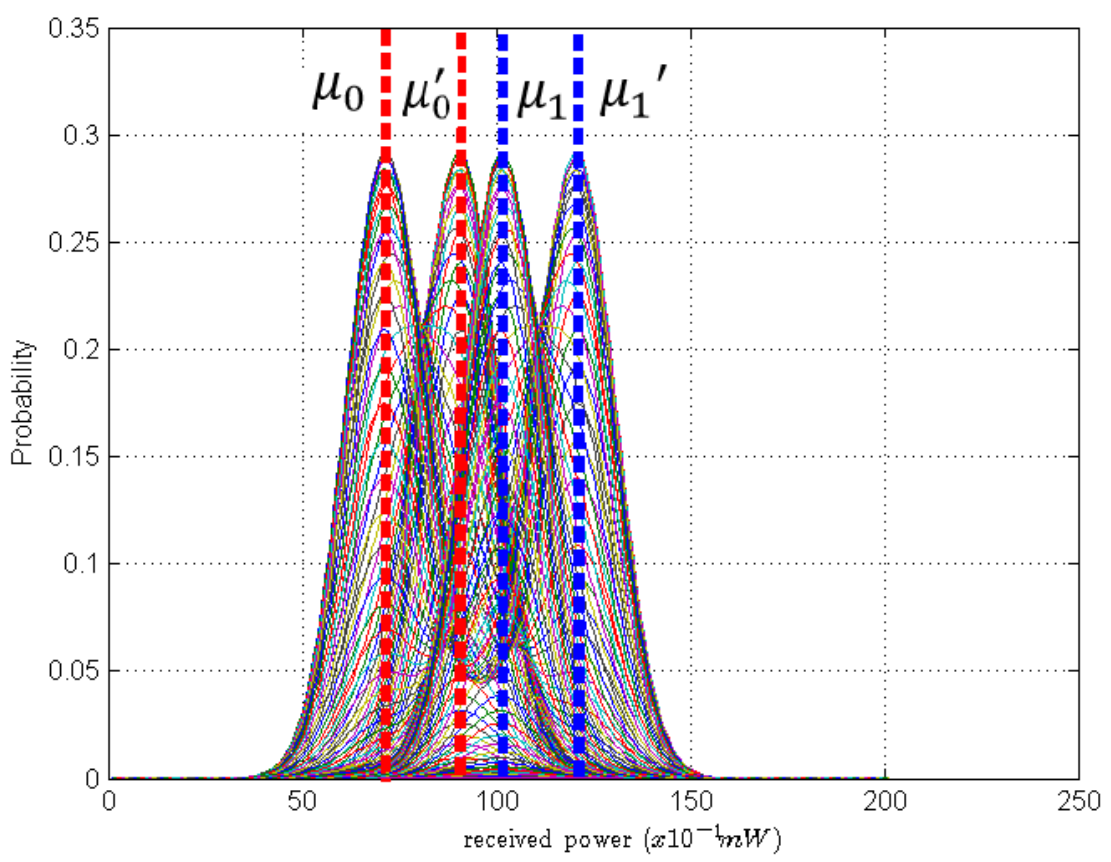

Figure 5.4: The PDF of moderate shadowing 
However, considering the fact that the shadowing is moderate, the two average values of receiving $s_{0}$ are close to each other which also applies for the case of receiving $s_{1}$. Therefore, we have two decision regions for the probability of error calculation.

With respect to severe shadowing, the average values of the two modes are shifted significantly from each other. In this case, we assume the shift is large enough to approximate the error by representing each mode as a normal distribution. We assume that when the separation, $S(t)>1$ between modes, then the distribution becomes two normal distributions as shown in Figure. 5.5.

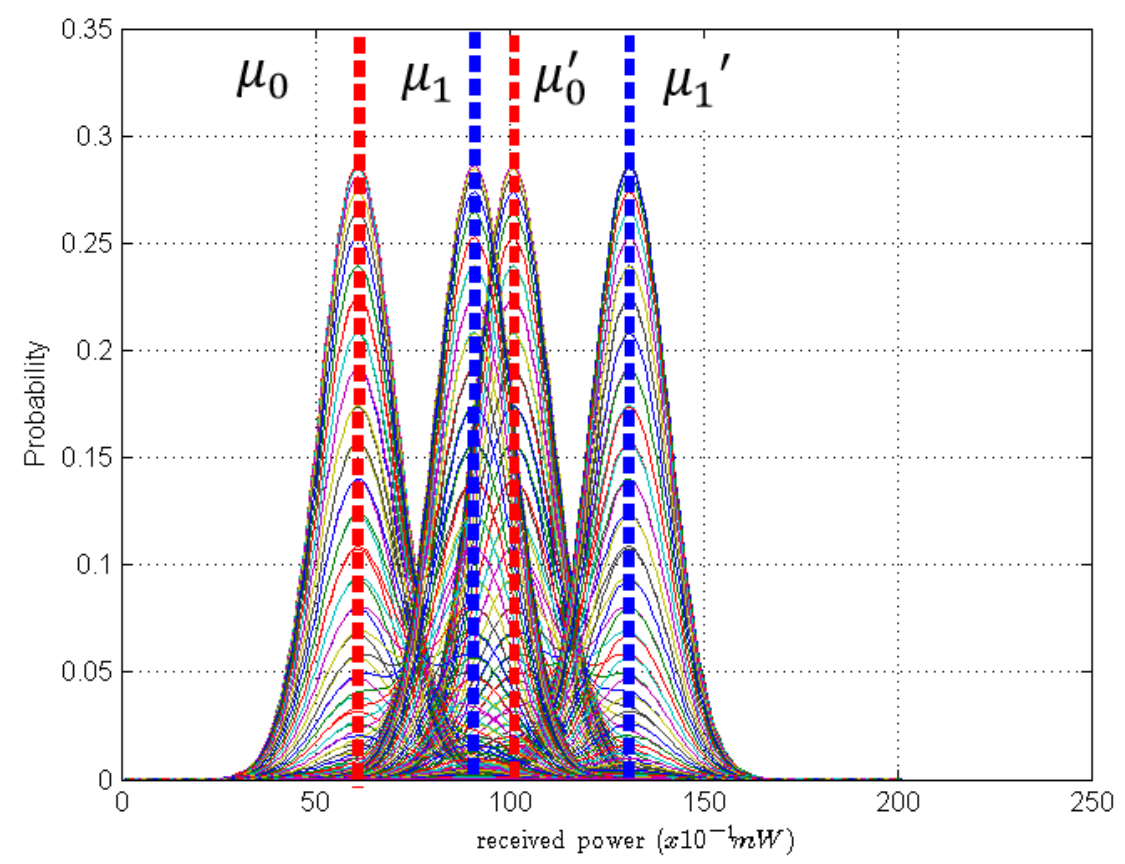

Figure 5.5: The PDF of severe shadowing

Moreover, due to the severe shadowing case, the Gaussian distribution of $s_{0}$ and $s_{1}$ becomes close to each other which significantly affects the probability of error which will be verified in the simulation section.

Furthermore, due to the obstruction effects, we will assume that the width of each Gaussian distribution is halved when compared to the case of no shadowing due to the transmitted signal power being distributed between the two paths around the obstructing 
object.

In the following, we derive the probability of error $P_{e}$

$$
\left.P_{e}=P(0) P(e \backslash 0)\right)+P(1) P(e \backslash 1)
$$

where $P(0)$ and $P(1)$ are the probability of transmitting " 0 " and "1" respectively, $P(e \backslash 0)$ and $P(e \backslash 1)$ are the conditional probability for receiving "1" while "0" was transmitted and receiving "0" while "1" was transmitted respectively, which is depicted by the shadowing in Figure. 5.3.

Due to symmetry, one can conclude that $P(e \backslash 0)=P(e \backslash 1)$.

As depicted in Figure. 5.3, the two Gaussian distributions have average values $\mu_{0}$, and $\mu_{1}$ representing the bits 0 and 1 respectively,

Define the decision region as

$$
x_{0}=\frac{\mu_{0}+\mu_{1}}{2}
$$

where $x_{0}$ is the mid-point between the two means as shown in Figure. 5.3.

Then $P(e / 0)$ is defined as

$$
P(e \backslash 0))=\int_{x o}^{\infty} \frac{1}{\sqrt{2 \pi \sigma^{2}}} \exp \left(\frac{-\left(x-\mu_{0}\right)^{2}}{2 \sigma^{2}}\right) \mathrm{d} x
$$

Also, $P(e \backslash 1)$ is defined as

$$
P(e \backslash 1)=\int_{-\infty}^{x_{0}} \frac{1}{\sqrt{2 \pi \sigma^{2}}} \exp \left(\frac{-\left(x-\mu_{1}\right)^{2}}{2 \sigma^{2}}\right) \mathrm{d} x
$$

Recall the $Q$ function as 


$$
Q_{x}=\int_{x}^{\infty} \frac{1}{\sqrt{2 \pi}} \exp \left(\frac{-y^{2}}{2}\right) \mathrm{d} y
$$

let $y=\frac{x-\mu_{0}}{\sigma}$, then, $d y=\frac{d x}{\sigma}$,

By Changing the integration limits, from (5.8), we will obtain $P(e / 0)$ as

$$
P(e \backslash 0)=\int_{\left(\frac{x_{0}-\mu_{0}}{\sigma}\right)}^{\infty} \frac{1}{\sqrt{2 \pi}} \exp \left(\frac{-y^{2}}{2}\right) \mathrm{d} y
$$

then

$$
P(e \backslash 0)=Q\left(\frac{x_{0}-\mu_{0}}{\sigma}\right)=Q\left(\frac{\frac{\mu_{0}}{2}+\frac{\mu_{1}}{2}-\mu_{0}}{\sigma}\right)
$$

Equation (5.12) can be simplified to

$$
P(e \backslash 0)=Q\left(\frac{\mu_{1}-\mu_{0}}{2 \sigma}\right)
$$

We can calculate $P(e \backslash 1)$ following same steps, where $P(e / 1)=Q\left(\frac{\mu_{1}-\mu_{0}}{2 \sigma}\right)$.

Recall (5.6), the probability of error for no shadowing will be given by

$$
P_{e}=Q\left(\frac{\mu_{1}-\mu_{0}}{2 \sigma}\right)
$$

To find the probability of error for moderate and severe shadowing when the intensity modulation is employed, the probability of error will be based on 4 average values due to the bimodal distribution as shown in Figure. 5.4 and Figure. 5.5. Then by applying the same procedure, as shown above, the probability of error for moderate and severe shadowing can 
be given by

$$
\begin{gathered}
P_{e}=2\left(Q\left(\frac{\mu_{1}-\mu_{0}}{4 \sigma}\right)+Q\left(\frac{\mu_{1}^{\prime}-\mu_{0}}{4 \sigma}\right)\right) \\
P_{e}=4\left(Q\left(\frac{\mu_{1}^{\prime}-\mu_{0}^{\prime}}{4 \sigma}\right)+Q\left(\frac{\mu_{0}-\mu_{1}^{\prime}}{4 \sigma}\right)+Q\left(\frac{\mu_{1}-\mu_{0}}{4 \sigma}\right)\right)
\end{gathered}
$$

Notice that in the case of moderate shadowing, we will still have two decision regions for detecting $s_{1}$ and $s_{0}$, while in the case of severe shadowing, the error probability is further increased as shown before due to the crossing of decision regions for detecting $s_{1}$ or $s_{0}$ which results in four decision regions.

\subsection{Simulations}

Matlab software was used to execute the simulation of the three cases of shadowing. We assumed Gaussian channel and the symbols $s_{1}$ or $s_{0}$ have equivalent variances but different means. Additionally, we are assuming intensity modulation is employed in the system where a stationary object with two main edges is obstructing the LOS signal, hence each modulation level will exhibit two modes. The error significantly increases when shadowing is in effect.

Figures. 5.3, Figure. 5.4, and Figure. 5.5 illustrate the density functions with the effects of shadowing. Here, blue and red lines represent modulation levels for $s_{1}$ and $s_{0}$, respectively.

Figures. 5.3 illustrates a clear distinction between each modulated intensity level without exhibiting dual modes, hence there may be an obstruction with a uniform surface resulting in low shadowing or even no shadowing.

Figures. 5.4 clearly shows the bimodal shadowing effect as there are two modes for each modulated intensity level. 
In Figures. 5.5, the overlap between the respective modes resulting from severe shadowing becomes visible. It is shown that the respective intensity levels begin to overlap.

Shadowing becomes more severe as shown in Figures. 5.5. Hence, the decision region of bits 0 and 1 significantly overlaps thereby greatly increasing the BER.

To simulate the probability of error for different cases of shadowing, we used Matlab by varying the received signal energies as well as its variances for the bimodal distribution. The simulation results clearly show that the error probability significantly increases due to the interleaving signal modes with each respective modulation.

Figure. 5.6 depicts the result of our simulation which compares the error probabilities with different shadowing effects. Clearly, the severe shadowing has the worst error probability when compared to the no (absent) and moderate shadowing cases. It is also clear that for all shadowing cases, when the received signal power is increased, as a result of increasing the LED transmitted power, the probability of error decreases.

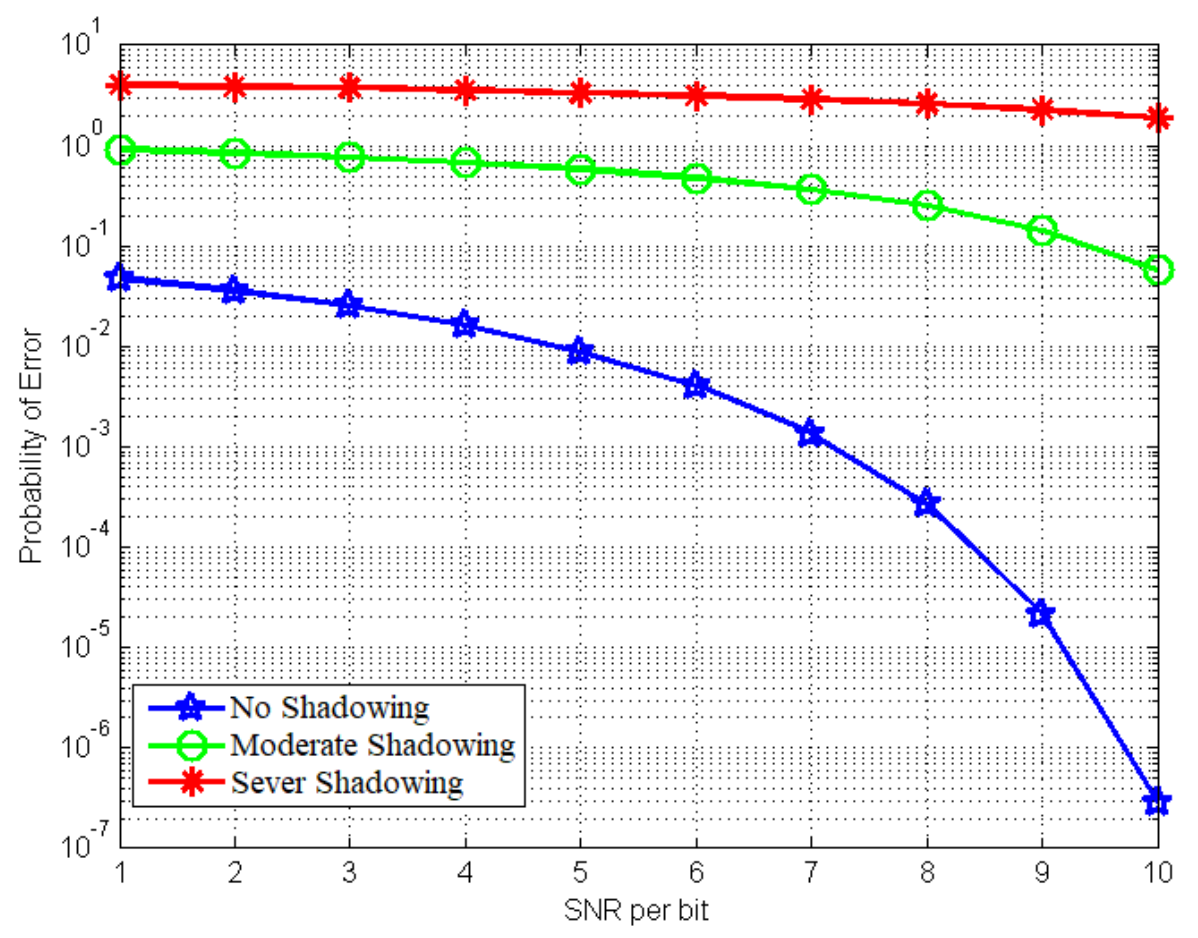

Figure 5.6: Error probability with different shadowing effects 


\subsection{Chapter Summary}

In this chapter, the shadowing effect on the V2V-VLC system is studied. We propose a method to take advantage of the optical diffraction phenomenon to overcome shadowing by employing a receiver with a wide FOV. We model the shadowing effect for visible light by a bimodal distribution and derived the probability of error for different types of shadowing. 


\section{Chapter 6}

\section{Sunlight Effect on V2V-VLC System and Denoising Schemes}

Its well-known fact that $47 \%$ of the total solar irradiance falls within the visible light frequency band of the spectrum [13] as shown in Figure. 6.1. The main meteorological conditions in network locations determine the level of sunlight. Therefore, the major challenge of the outdoor VLC system is the strong influence of the ambient-light noise because of sunlight. Sunlight represents unmodulated sources, that can be received at an average power much larger than the desired signal, even when optical filtering is employed. However, the noise due to the solar irradiance and other surrounding light sources, is a major concern that degrades the performance of the VLC system in outdoor application in terms of SNR and BER.

In this chapter, the effect of solar irradiance and other external sources are investigated for V2V-VLC system with regard to SNR, BER, and data rate. Then, we propose two schemes to combat the effect of sunlight on the V2V-VLC system. Firstly, we propose the differential receiver as an efficient denoising scheme, then, we propose a kNN-algorithmmachine learning-based adaptive filter scheme. In the second scheme, our smart system can adapt itself according to varying noise conditions and help to achieve acceptable BER in support of reliable communications. 


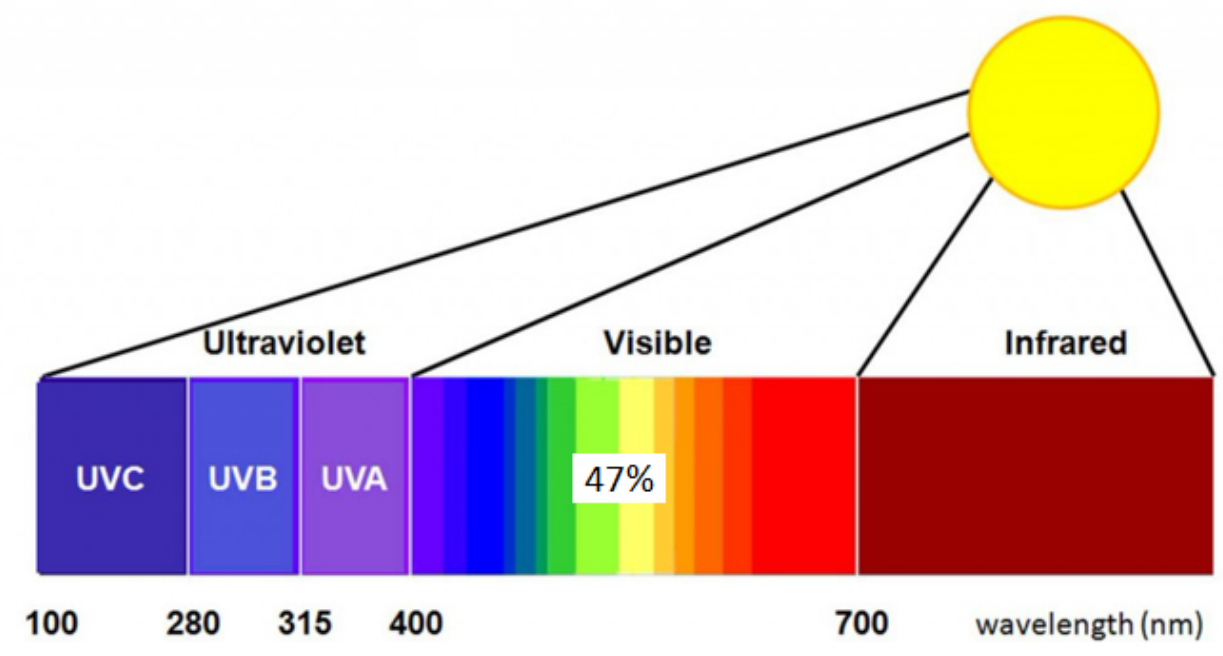

Figure 6.1: Sunlight spectrum [8]

\subsection{Solar Irradiance}

Solar irradiance defines as the amount of solar power in any place. The earths rate of rotation, the difference in local longitude, and the standard meridian for the local time zone are considered the main keys in irradiance calculations. Measuring irradiance correctly needs high accurate apparatuses. In general, solar irradiance can be calculated from clear sky solar radiation on a horizontal surface in $W / m^{2}$ [94].

Most important parameter to measure direct solar radiation (irradiance) is $q$ factor and is given by [94]

$$
q_{\text {sun }}=\left(1350.3\left[1+0.099 \cos \left(\frac{360 n}{365}\right)\right][\sin \varphi \sin \zeta+\cos \varphi \cos \zeta \cos \omega]\right)
$$

where $\zeta$ is solar declination, $\omega$ is the angular displacement, $\varphi$ is the location longitude, and $n$ is the day of the year.

However, calculating solar irradiance is beyond the scope of this work, instead, the effect of solar irradiance on the V2V-VLC system will be addressed. 


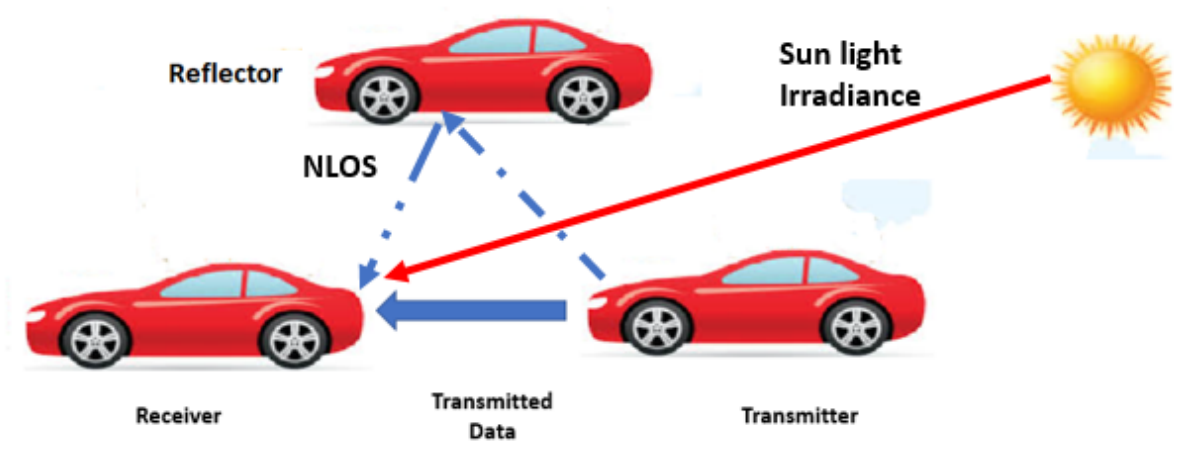

Figure 6.2: The effect of solar irradiance on V2V-VLC

\subsection{SNR of V2V-VLC System}

In this work, we consider the system given in section 2.2. Also, we choose the city of Toronto as a reference to calculate solar irradiance during a full year (12 months) period to cover the worst and best cases of irradiance. We assume the sunlight incident angle within the FOV of the receiver all the time. Furthermore, OOK modulation scheme is used. The values of solar irradiance are given in Table 6.1, while Table 6.2 gives classes of natural light in different metrological condition and different time.

Figure. 6.2 shows the scenario of solar irradiance effect on V2V-VLC, while Figure. 6.3 shows the response of the APD of predicted spectral irradiance of the sunlight for different visible light wavelength in the city of Toronto. Here, APD response is given without any optical filter scheme. To show the strong effect of the solar irradiance on the V2V-VLC system, we calculate the SNR for the V2V-VLC system without any denoising scheme. Figure. 6.4 shows the strong effect of sunlight without any filtering scheme on SNR of the system during different months of the year. The summer's months shows worst SNR, where SNR approaches $\simeq-3 d B m$, and in winter, SNR approaches $\simeq 9 \mathrm{dBm}$. 
Table 6.1: Solar irradiance at the city of Toronto [1]

\begin{tabular}{|c|c|}
\hline \hline & $\begin{array}{c}\text { Average Solar Insolation } \\
\text { Measured in } \mathrm{kWh} / \mathrm{m}^{2} / \text { day } \\
\text { with Solar Panel direction } \\
\text { southwest } 45^{\circ} \text { from south }\end{array}$ \\
\hline January & 1.5 \\
\hline February & 2.35 \\
\hline March & 3.29 \\
\hline April & 4.35 \\
\hline May & 5.12 \\
\hline June & 5.88 \\
\hline July & 5.87 \\
\hline August & 5.02 \\
\hline September & 3.92 \\
\hline October & 2.64 \\
\hline November & 1.55 \\
\hline December & 1.24 \\
\hline \hline
\end{tabular}

Table 6.2: Classes of natural light [2].

\begin{tabular}{|c|c|}
\hline \hline Natural Light Class & Intensity (lx) \\
\hline Sunlight not considered & 0 \\
\hline Clear night, full moon & 0.3 \\
\hline Winter's day, overcast sky & $900-2000$ \\
\hline Summer's day, overcast sky & $4000-20000$ \\
\hline Winter's day, clear sky & Up to 9000 \\
\hline Summer's day, clear sky & Up to 100000 \\
\hline
\end{tabular}




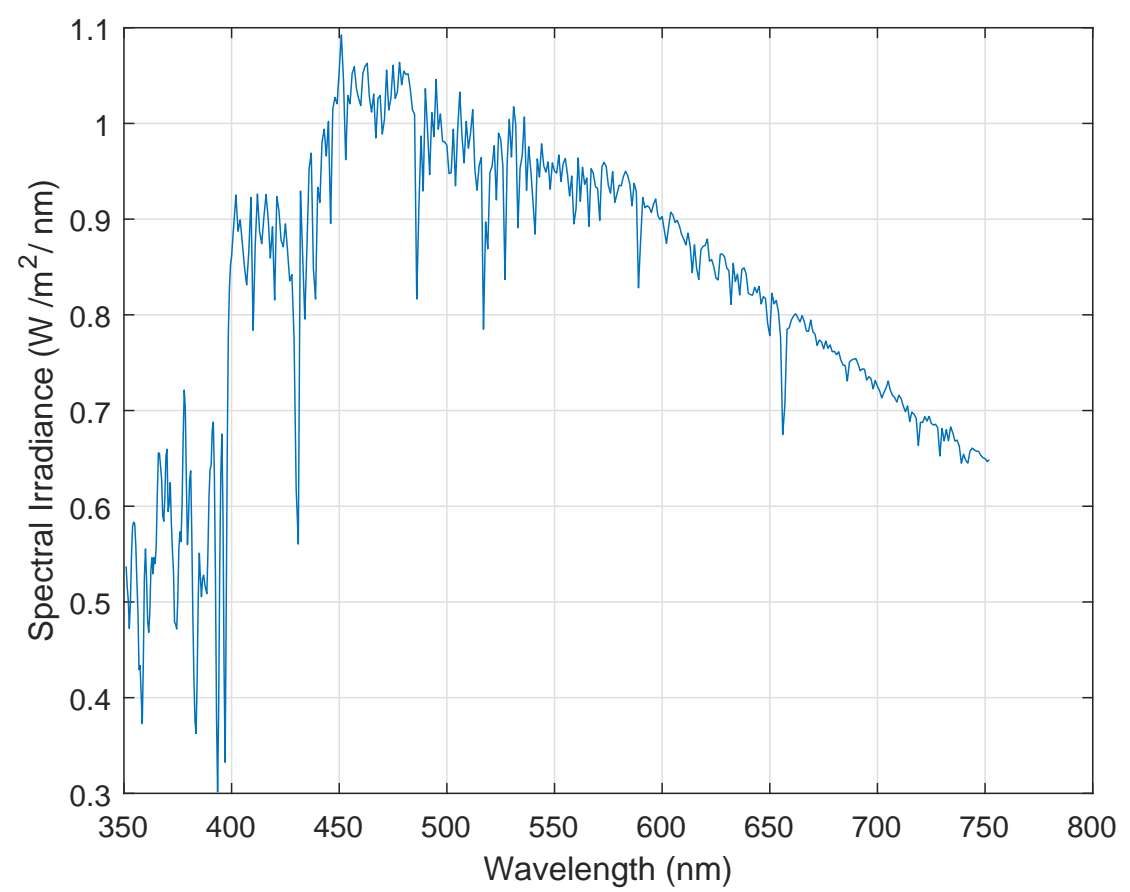

Figure 6.3: Average predicted solar irradiance at the city of Toronto in month of June [1].

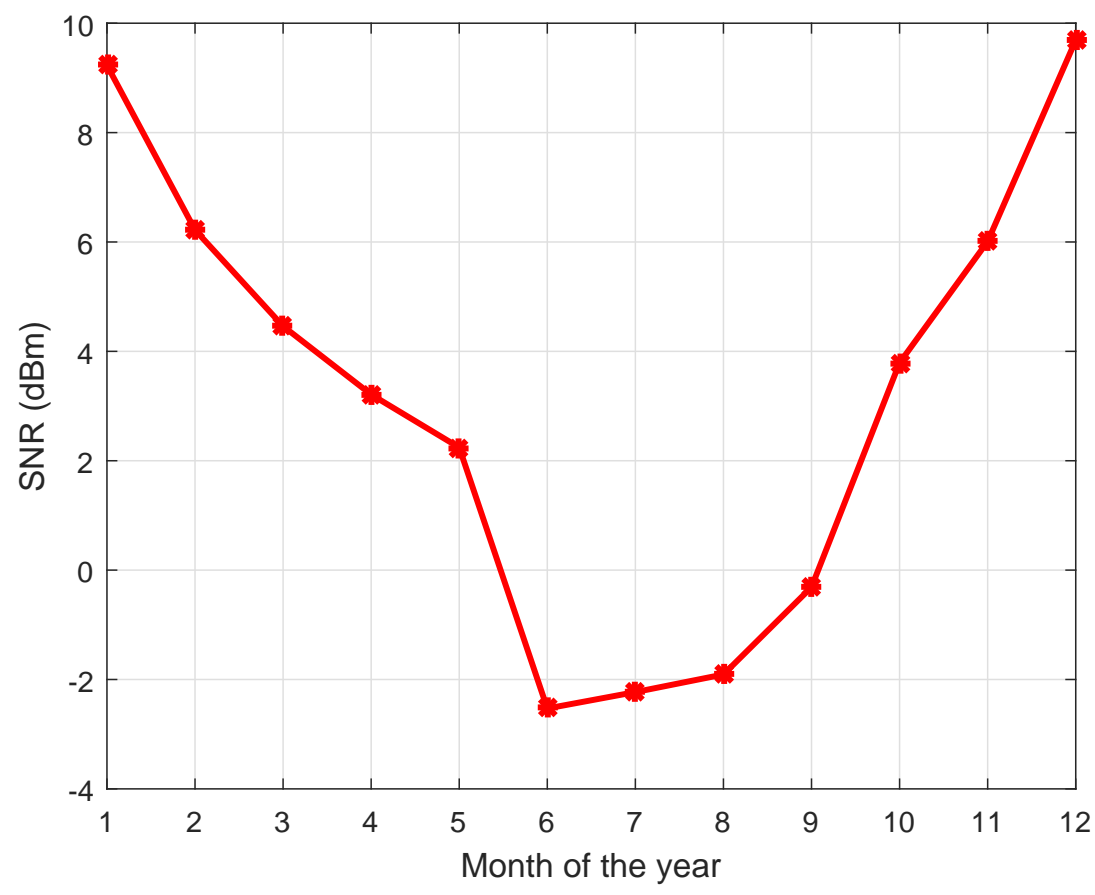

Figure 6.4: SNR without any filtration scheme at the city of Toronto when the LED illuminance intensity is $1750(c d)$ [1]. 


\subsection{Related Work in Denoising Schemes for VLC}

Most of the previous works for the outdoor-VLC system did not consider the effect of a practical ambient light noise which varies from time to time throughout the year, and from location to location. Few work has been addressed the effect of sunlight in the outdoor-VLC system. In [13], the authors considered the impact of sunlight as a function of location, time and for different surfaces over the four seasons of the year and analyzed the solar irradiance. Moreover, they investigated the effect of solar irradiance in the outdoor-VLC system in terms of data rate and BER degradation. The authors in [95] presented a daylight noise model based on a modified Blackbody radiation model to capture the effect of ambient-light noise. Also, they presented a new receiver using the selective combining technique to reduce the effect of background noise. The authors in [41] proposed a measurement-based time variant non-clear sky channel model for the I2V-VLC system which considers the dynamic characteristics of background radiation to enable more realistic and accurate prediction of the VLC system performance on the outdoor application. Moreover, they introduced a new receiver design with dual-reception and effective ambient-light rejection capabilities which employs the selection diversity technique in order to mitigate the impact of ambient-light noise due to daylight. In [96], the authors presented an analytical analysis of solar irradiance. They investigated the effect of solar irradiance on the VLC system by using optical filter in terms of data rate and BER degradation.

MLE has also been applied to the VLC field. The authors in [97] showed the applicability and feasibility of different MLE techniques based on iris recognition through smart phone captured images. Authors trained different classifiers and used histogram equalization processes to maximize accuracy. In [98], the authors proposed a novel object tracking framework based on an online learning scheme which can work robustly in challenging scenarios. They also proposed, a learning-based particle filter with color and edge-based features. Moreover, a multiple classifiers fusion localization techniques using the received signal strengths of visible light is proposed in [99] in which, LEDs are used to transmit various 
intensity modulated sinusoidal signals to be received by a PDs placed at various grid points.

\subsection{Noise Calculations}

At the receiver, the received signal consists of the transmitted signal and the noise signal. The photocurrent $I_{o}$ due to the received optical power consists of two components, the LOS component and the NLOS component, i.e, $\left(I_{o}=I_{o_{L O S}}+I_{o_{N L O S}}\right)$ and is given as

$$
I_{o}=G A_{r}\left(\int_{\lambda_{1}}^{\lambda_{2}} P_{r}^{L O S}(\lambda) \gamma(\lambda) T_{o}(\lambda) d \lambda+\int_{\lambda_{1}}^{\lambda_{2}} P_{r}^{N L O S}(\lambda) \gamma(\lambda) T_{o}(\lambda) d \lambda\right)
$$

where $\lambda_{1}, \lambda_{2} \in[350750](n m)$ are the visible light wavelengths, $P_{r}^{L O S}(\lambda), P_{r}^{N L O S}(\lambda)$ are the received power and are given in (2.38), and (2.39) respectively for certain wavelength $\lambda, G$ is $\mathrm{APD}$ gain, $\gamma(\lambda)$ is the responsivity of the APD given in $\mathrm{A} / \mathrm{W}, A_{r}$ is the effective area of the receiver, and $T_{o}(\lambda)$ is the transmittance of the bandpass optical filter.

Similarly, the photocurrent $I_{s}$ produced at APD due to the solar irradiance is given as

$$
I_{s}=G A_{r} \cos \left(\theta_{\text {sun }}\right) \int_{\lambda_{1}}^{\lambda_{2}} P_{s}(\lambda) \gamma(\lambda) T_{o}(\lambda) d \lambda
$$

where $P_{s}(\lambda)$ is the solar irradiance given in $W / m^{2} / n m, \theta_{\text {sun }}$ is the incident angle of the sunlight on the receiver surface.

The random arrival of the incident photons from both LED light, and sunlight result into shot noise. This type of noise can be modeled by Poisson process. Moreover, when the number of incident photons is large, shot noise is approximated by a Gaussian process. (using central limiting theorem). 
According to [15] the shot noise variance is given as

$$
\sigma_{\text {Shot }}^{2}=2 q_{e} G^{2} F\left(I_{o}+I_{s}\right) B
$$

where $q_{e}$ is the electron charge, $F$ is the excess noise; $F=k_{n} G+\left(2-\frac{1}{G}\right)\left(1-k_{n}\right)$ where $k_{n}$ is the holes/electrons ionization rate [100], and $B$ is the APD bandwidth.

\subsubsection{Other Noise Sources}

In addition to the shot noise, there are many different types of noises degrading the performance of the VLC system such as dark noise. Dark noise is caused due to avalanche effect of the APD and is given as [15]

$$
\sigma^{2}{ }_{\text {Dark }}=2 q_{e} G^{2} F I_{d g} B+2 q_{e} I_{d s}
$$

where $I_{d s}$ is the surface dark current and $I_{d g}$ is the bulk dark current that experiences the avalanche effect of the APD.

Also, due to the random thermal motion of charge carriers, thermal noise is generated, the variance of thermal noise is given as [15]

$$
\sigma_{\text {Thermal }}^{2}=4\left(\frac{K_{B} T_{k}}{R_{L}}\right) F_{n} B
$$

where $K_{B}$ is Boltzmann constant, $T_{k}$ is the temperature in Kelvin, $R_{L}$ is the load resistance given as $50 \Omega$, and $F_{n}$ is the photodiode noise figure.

Due to variances summation principle which is given in section (1.3), the total noise variance is given by

$$
\sigma_{n}^{2}=\sigma_{\text {Shot }}^{2}+\sigma_{\text {Dark }}^{2}+\sigma_{\text {Thermal }}^{2}
$$

In this work, we consider Hamamatsu S8664 model for APD [21]. The parameters and 
their values are given in Table. 1.2.

\subsubsection{Performance Metrics}

The SNR for the system is

$$
S N R=\frac{I_{o}}{\sigma_{n}^{2}}
$$

The maximum achievable data rate defined as

$$
R_{\max }=B \log _{2}(1+S N R)
$$

For OOK modulation scheme, BER for certain SNR is given by (2.44).

\subsection{Differential Receiver as a Denoising Scheme to Improve the Performance of V2V-VLC Systems}

Achieving high SNR, high data rate, and minimizing multipath dispersion are the most challenges during the receiver design for any system. In order to design an efficient optical filtering system for the outdoor V2V-VLC system in the presence of sunlight and other artificial lights, eye safety should additionally be taken into account. Many previous works considered bandpass blue optical filter for denoising process [96], [101]. Such schemes include APD with collimate lens on the top of the APD to focus incident light onto the surface of APD. The optical filter is laid on this lens for blocking unwanted lights as shown in Figure. 6.5.

In this section, a differential receiver with optical filter scheme is proposed as a solution to combat the effect of the sunlight noise. We investigate the system performance for three scenarios: without optical filter, with optical filter, and with the proposed differential receiver. Simulation results show that differential receiver can predict about $60 \%$ of solar irradiance 


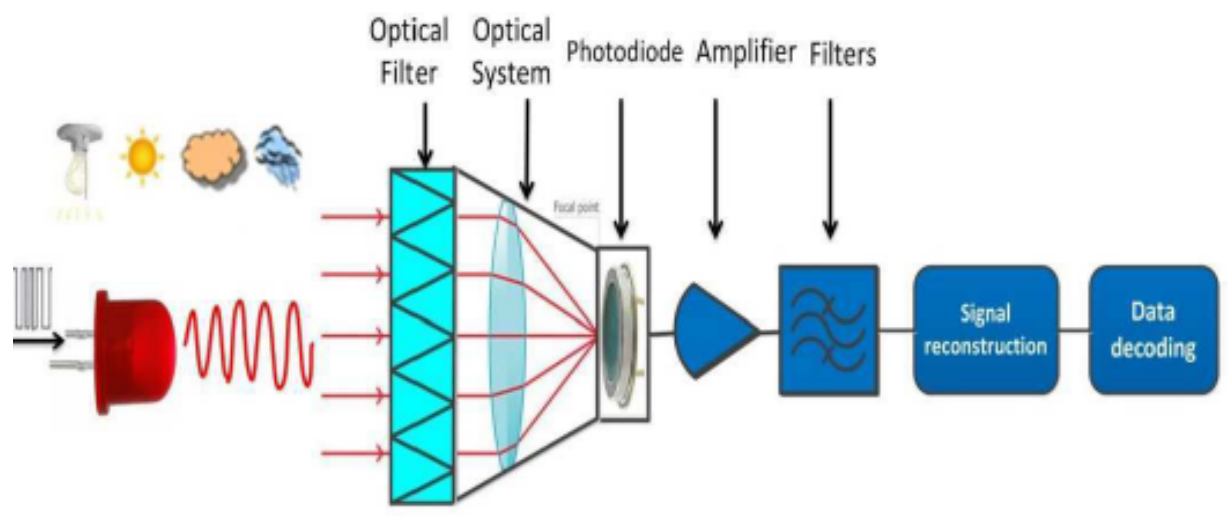

Figure 6.5: The receiver architecture of the VLC system.

and can improve the BER from $5 \times 10^{-4}$ up to $5 \times 10^{-5}$. Moreover, the results depict the V2V-VLC system can still work under solar irradiance where the worst achievable BER during June is $\simeq 5 \times 10^{-4}$.

\subsubsection{Differential Filtering Scheme for $2 \times 2$ MIMO-V2V-VLC}

Differential filtering scheme is a well-known idea in communication field [102], [103]. In this work, we apply this scheme with some modifications to the system to mitigate the effect of sunlight noise. The differential filtering circuit is shown in Figure. 6.6. Each transmitter source transmits with different wavelength, i.e., transmitter 1 uses $\lambda_{1}$ and transmitter 2 uses $\lambda_{2}$, where the difference between $\lambda_{1}$ and $\lambda_{2}$ is several tens of nanometers, thus, we can assume the channel coefficients for two transmitters are equal. We consider red shift phenomena to adjust the wavelengths $\lambda_{1}$ and $\lambda_{2}[104]$.

For $2 \times 2$ MIMO, the received signal at any receiver $\mathbf{y}_{\mathbf{r i}}$ is give by

$$
\mathbf{y}_{\mathbf{r i}}=\left[\begin{array}{ll}
h_{i 1} & h_{i 2}
\end{array}\right]\left[\begin{array}{ll}
\mathbf{x}_{\mathbf{1}} & \mathbf{x}_{\mathbf{2}}
\end{array}\right]^{\mathbf{T}}+\mathbf{n}_{\mathbf{i}}
$$

where $(i=1,2), \mathbf{x}_{\mathbf{1}}, \mathbf{x}_{\mathbf{2}}$ indicate the transmitted vector of first and second transmitter respectively with wavelength $\lambda_{1}$, and $\lambda_{2} . \mathbf{n}_{\mathbf{i}}$ indicates the noise vectors including shot noise, 


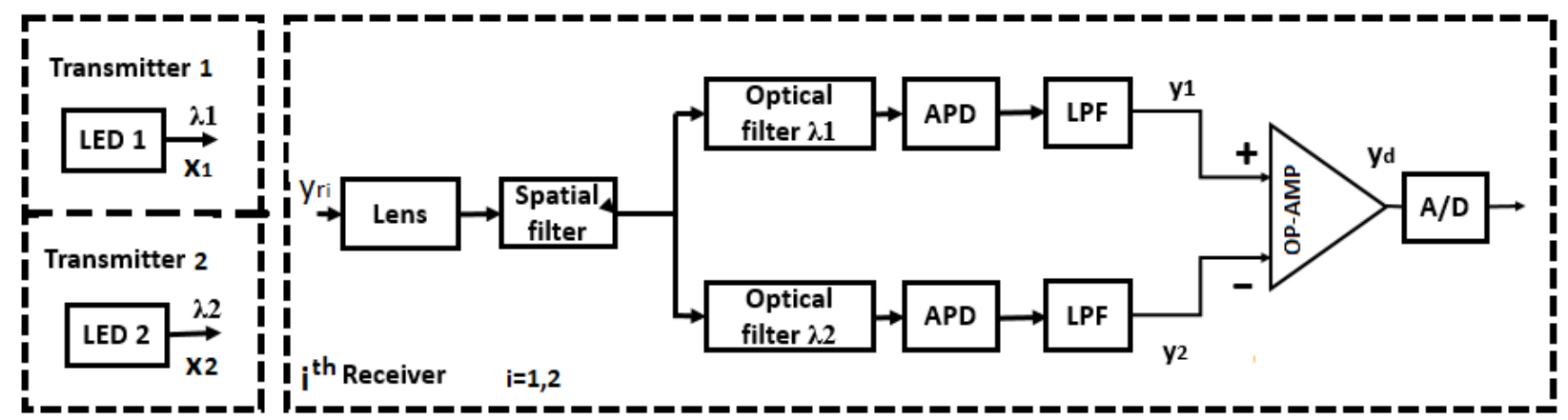

Figure 6.6: Denoising differential scheme of VLC.

which is rising up from the sunlight in addition to thermal noise and dark noise. We will assume only the shot noise, as it is the dominant noise.

The received signal is collected by a lens as shown in Figure. 6.6. Thereafter, the signal passes to a spatial filter to alter the structure of light. Then, the signal passes through two parallel bandpass optical filters with equal bandwidth but different centre frequencies $\left(f_{1}=\frac{c}{\lambda_{1}}, f_{2}=\frac{c}{\lambda_{2}}\right)$, where $c$ is the speed of light. Afterward, the outputs of the optical filters pass to APDs and then the signal passes through Low Pass Filter (LPF). At the end, two parallel signals $\mathbf{y}_{\mathbf{1}}$, and $\mathbf{y}_{\mathbf{2}}$ are subtracted by an operational amplifier (OP-AMP) to form one signal $\left(\mathbf{y}_{\mathbf{d}}=\mathbf{y}_{\mathbf{1}}-\mathbf{y}_{\mathbf{2}}\right)$ to pass to Analog-to-Digital (A/D) converter, and finally to the signal detection process.

Since the optical filter will pass either $\mathbf{x}_{\mathbf{1}}$, or $\mathbf{x}_{\mathbf{2}}$ depending on the signal wavelength, $\mathbf{y}_{\mathbf{1}}$, and $\mathbf{y}_{\mathbf{2}}$ are given by

$$
\begin{aligned}
& \mathbf{y}_{1}=\mathbf{H}_{\mathbf{T}} \mathbf{x}_{1}+\mathbf{n}_{1} \\
& \mathbf{y}_{\mathbf{2}}=\mathbf{H}_{\mathbf{T}} \mathbf{x}_{\mathbf{2}}+\mathbf{n}_{\mathbf{2}}
\end{aligned}
$$

where $\mathbf{H}_{\mathbf{T}}$ is the transfer function of the channel of one branch of the differential receiver. In this work, $\mathbf{H}_{\mathbf{T}}$ can be considered the same for two parallel branches. 
The output of the OP-AMP, $\mathbf{y}_{\mathbf{d}}$ is given by

$$
\begin{aligned}
\mathbf{y}_{\mathbf{d}} & =\mathbf{y}_{\mathbf{1}}-\mathbf{y}_{\mathbf{2}} \\
& =\mathbf{H}_{\mathbf{T}} \mathbf{x}_{\mathbf{d}}+\mathbf{n}_{\mathbf{d}}
\end{aligned}
$$

where $\left(\mathbf{x}_{\mathbf{d}}=\mathbf{x}_{1}-\mathbf{x}_{2}\right)$, and $\left(\mathbf{n}_{\mathrm{d}}=\mathbf{n}_{1}-\mathbf{n}_{\mathbf{2}}\right)$.

\subsubsection{Signal Filtration by Differential Receiver}

Assuming the sunlight noise is a stochastic stationary random process. The background noise component after the LPF will be cancelled due to the differential detection system.

The sunlight noise spectrum, whose envelope denotes by $S$, is spread over a very large spectral band $B_{s}$. Because we assume each headlight transmit signal with different $\lambda$, we need two Band Pass Filter (BPF) (optical filters) at suggested differential detector with central frequencies $f_{1}$ and $f_{2}$. Their impulse response functions are $h_{1}(t)$ and $h_{2}(t)$, respectively and the corresponding transfer functions are $H_{1}(f)$ and $H_{2}(f)$, respectively. Also, let the two filters have same bandwidth given by $B_{f}$. Since $B_{s}>>B_{f}$, the output of the filters $S_{1}$ and $S_{2}$ can be considered flat.

Assuming the optical filter is a linear time-invariant system, $S_{1}$ and $S_{2}$ are given by

$$
S_{i}(t)=\int_{-\infty}^{\infty} h_{i}(\tau) S(t-\tau) d \tau \quad i=1,2 .
$$

The filters output $S_{1}$ and $S_{2}$ are passed to the APD. At the output of $i^{\text {th }} \mathrm{APD}$, the light intensity is given by

$$
I_{S i}(t)=\int_{T_{s}}\left|S_{i}(t)\right|^{2} d t
$$

where $T_{s}$ is the symbol period of the assigned modulation scheme. 
Hence, $\left|S_{1}(t)\right|^{2}$ is given by

$$
\left|S_{1}(t)\right|^{2}=\int_{-\infty}^{\infty} \int_{-\infty}^{\infty} h_{1}\left(\tau_{1}\right) S\left(t-\tau_{1}\right) h_{1}\left(\tau_{2}\right) S\left(t-\tau_{2}\right) d \tau_{2} d \tau_{1}
$$

For first APD, substitute (6.16) in (6.15), we get

$$
I_{S 1}(t)=\int_{-\infty}^{\infty} \int_{-\infty}^{\infty} h_{1}\left(\tau_{1}\right) h_{1}\left(\tau_{2}\right) \int_{T_{s}} S\left(t-\tau_{1}\right) S\left(t-\tau_{2}\right) d \tau_{2} d \tau_{1} d t
$$

Since $S$ is considered to be an ergodic stationary random process, then the third integral in (6.17) is the autocorrelation function of $S(t)$. Thus, we can write it in terms of expected value $E[$.$] as$

$$
E\left(S\left(t-\tau_{1}\right) S\left(t-\tau_{2}\right)\right)=\int_{T_{s}} S\left(t-\tau_{1}\right) S\left(t-\tau_{2}\right) d t=R_{s}\left(\tau_{2}-\tau_{1}\right)
$$

Assuming that $H_{1}$ is an ideal BPF, its impulse response is $h_{1}(t)=\operatorname{sinc}\left(2 \pi B_{f} t\right) \cos \left(2 \pi f_{1} t\right)$. The impulse response $h_{1}(t)$ is a sinc function with its first zero at $\frac{1}{\Delta f}\left(\Delta f=f_{2}-f_{1}\right)$, modulated by $\cos \left(2 \pi f_{1} t\right)$. On the other hand, assuming a rectangular-shaped Power Spectral Density (PSD) for $\mathrm{S}$ (just for the sake of demonstration simplicity), its autocorrelation function will be a sinc function with its first zero at $\frac{1}{B_{s}}$. Given that $B_{s}>>\Delta f$ in practice, this autocorrelation can relatively be considered as a Dirac delta function. In other words, $\mathrm{S}$ can be considered as a white noise in the calculation of $I_{S 1}$. Figure. 6.7 shows an illustration for the PSD of the background noise. Also, it shows bandpass filtering of bandwidth $B_{f}$ around $f_{1}$ and $f_{2}$, corresponding to the bandpass background noises field envelopes $S_{1}$ and $S_{2}$.

The autocorrelation function in (6.18) can be given by

$$
R_{s}\left(\tau_{2}-\tau_{1}\right)=\sigma_{s}^{2} \delta\left(\tau_{2}-\tau_{1}\right)
$$




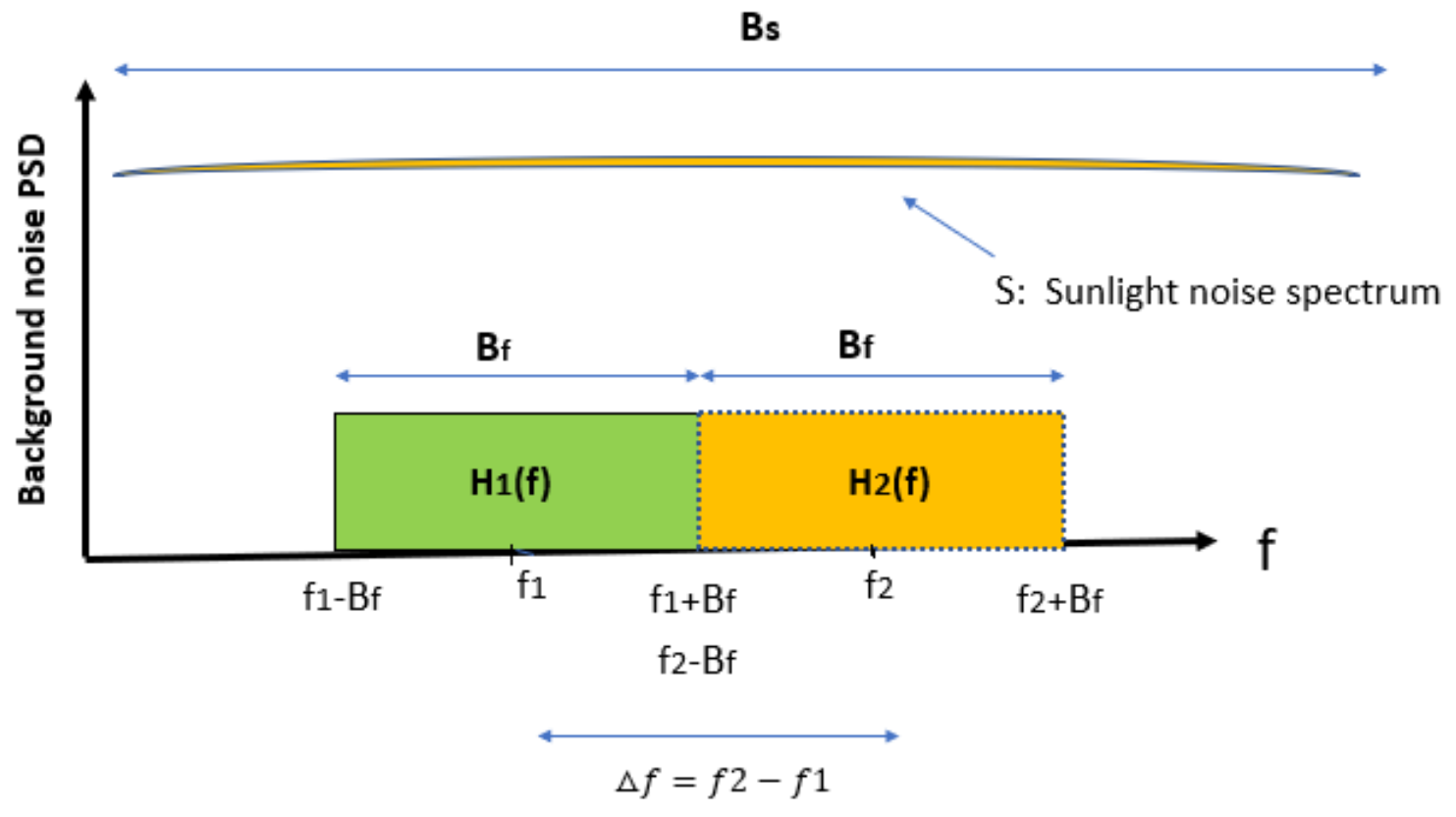

Figure 6.7: Background noise PSD.

where $\sigma_{s}^{2}$ is the variance of the noise and $\delta$ is Dirac delta function.

Furthermore, (6.15) can be written as

$$
I_{S 1}(t)=\sigma_{s}^{2} \int_{-\infty}^{\infty} h_{1}^{2}(\tau) d \tau
$$

Considering both filters are identical, at the operational amplifier (OP-AMP), recall Figure. 6.6, two light intensities $I_{S 1}$ and $I_{S 2}$ will be subtracted, thus

$$
I_{S 1}-I_{S 2}=\sigma_{s}^{2} \int_{-\infty}^{\infty}\left[h_{1}^{2}(\tau)-h_{1}^{2}(\tau)\right] d \tau
$$

This is what we had denoted by $n_{d}$ in (6.13). Using the Parseval equality, we obtain

$$
n_{d}=\sigma_{s}^{2} \int_{-\infty}^{\infty}\left[\left|H_{1}(f)\right|^{2}-\left|H_{2}(f)\right|^{2}\right] d f=0
$$

Equation (6.22) indicates that the shot noise, which is rises by the sunlight, in V2V-VLC can be neglected by using differential detector. 


\subsubsection{Simulation Results}

We use Matlab to simulate the proposed system and consider the city of Toronto for calculating solar irradiance. Simulation parameters are given in Tables. 1.2, 2.1, 3.1, and 6.1.

Figure. 6.8 shows the response of the APD of the predicted spectral irradiance of the sunlight for different visible light wavelength. Here, APD response is given for three cases: without optical filter, with blue optical filter, and with differential receiver. Moreover, Figure. 6.8 shows that the violet and blue lights have less irradiance, while yellow and green lights have the highest irradiance. Differential receiver capture up to $60 \%$ of solar irradiance while blue filter capture up to $75 \%$, which means the proposed scheme shows good improvement in the system performance.

The proposed system performance is studied by investigating the average BER versus the sunlight irradiance for different months of the year for the three scenarios as shown in Figure. 6.9. A significant improvement is achieved when the differential receiver and blue

filter are used. Differential receiver can improve the BER up to $\simeq 5 \times 10^{-5}$, while blue filter scenario can improve the BER up to $\simeq 1 \times 10^{-4}$.

Figure. 6.10 shows the data rate versus the sunlight irradiance for the three considered scenarios. It is shown that the proposed scheme improves the data rate three times comparing without any filtering scheme, and by one and half time compared to traditional filtering scheme. It is also shown by Figure. 6.9 and Figure. 6.10 that the V2V-VLC system can still work under solar irradiance, where the worst achievable BER during June (highest irradiance value) is $\simeq 5 \times 10^{-4}$. 


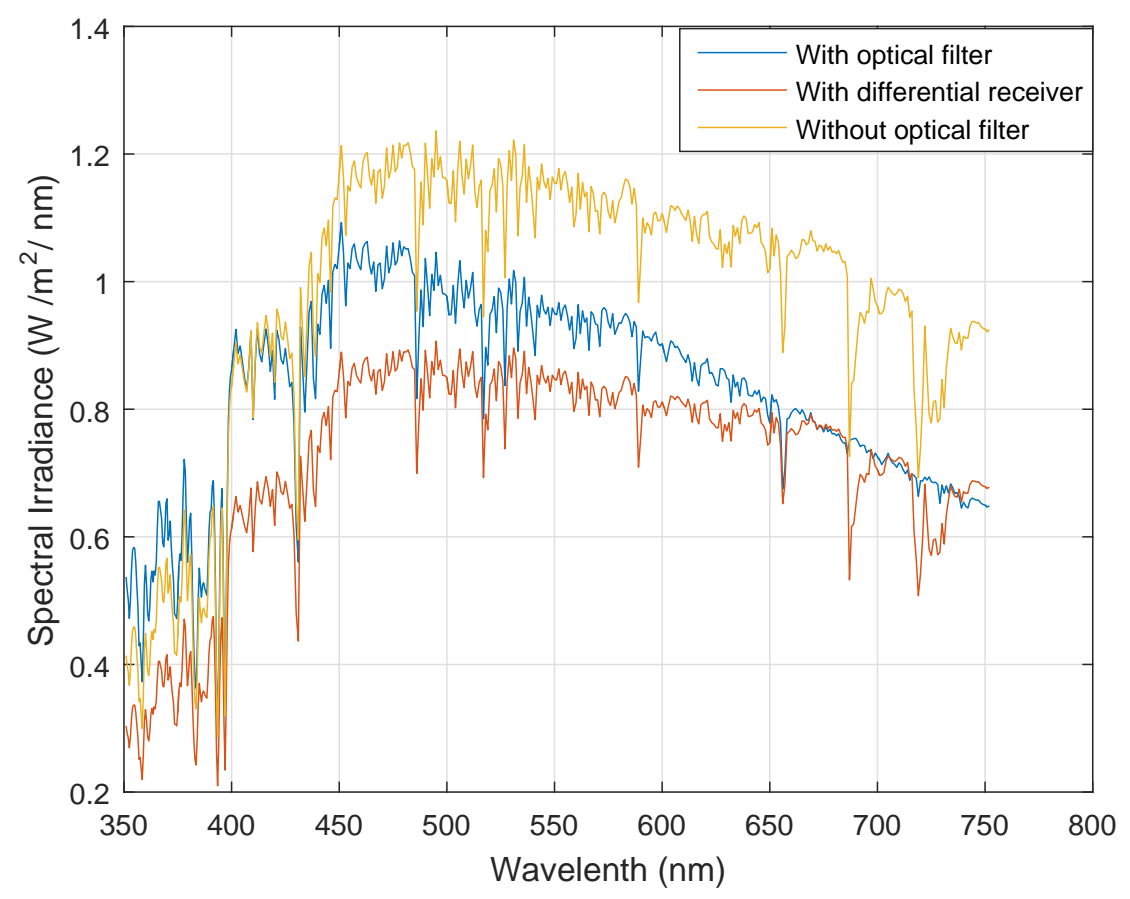

Figure 6.8: Average predicted solar irradiance at Toronto city in month of June with the denoising schemes.

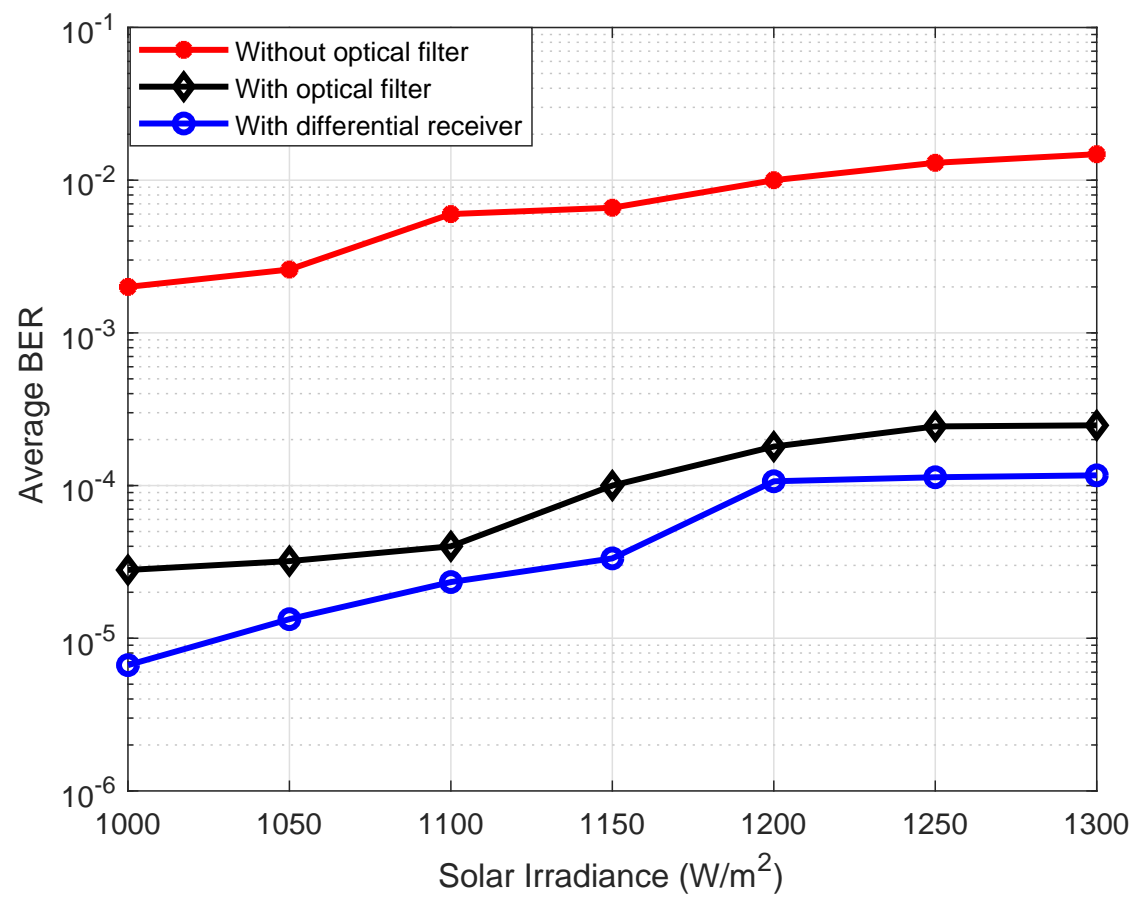

Figure 6.9: BER with sunlight irradiance considering city of Toronto. 


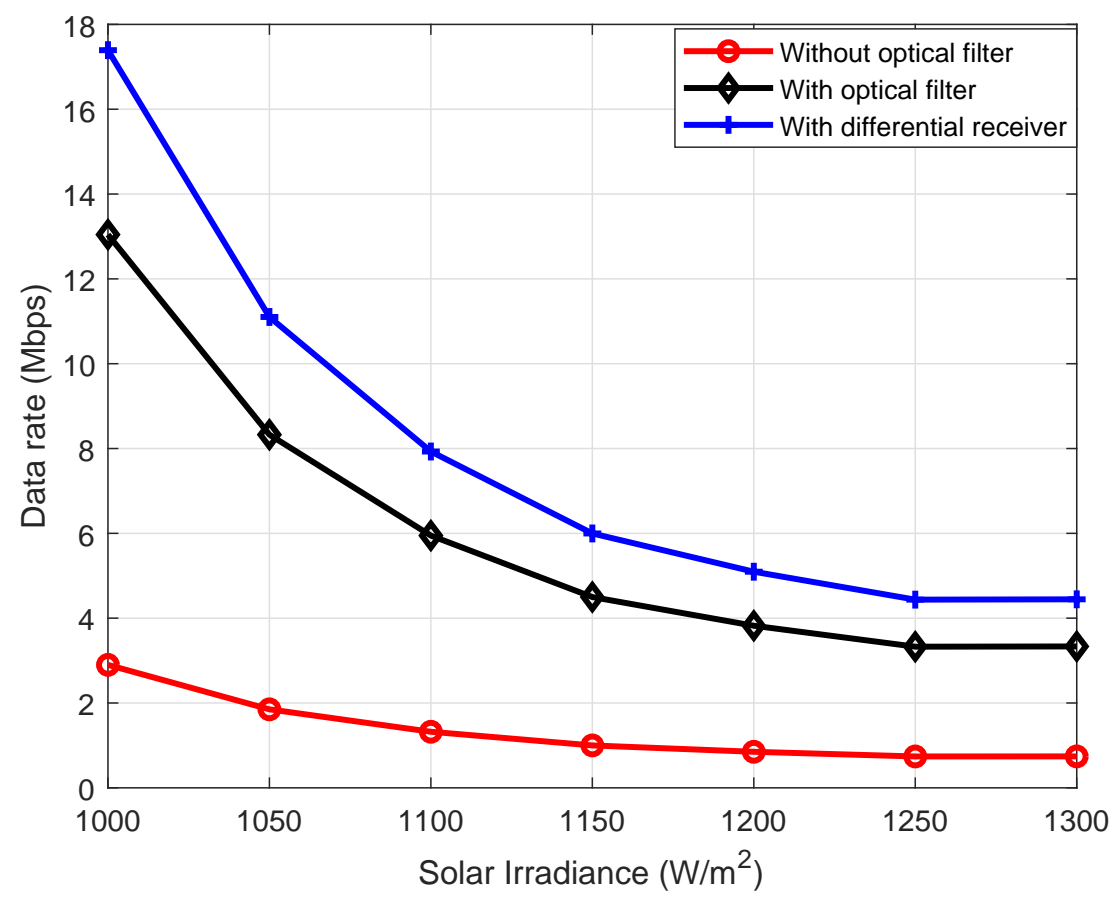

Figure 6.10: Achievable data rate with sunlight irradiance considering city of Toronto.

\subsection{Denoising Scheme to Improve the Performance of V2V-VLC Systems by Machine Learning}

Machine learning (MLE) is a powerful sub-field of Artificial Intelligence and is being used for model training purposes in data mining, pattern recognition, and image processing. Centralized and distributed algorithms are required for data fusion and automated decision making during smart sensing/monitoring. MLE is used to develop technologies for machines/devices, for monitoring present behavior and predicting future behaviors. It has also been utilized in optical communication systems for indoor localization and failure prediction.

MLE techniques are becoming pervasive and they can play a key role in all stages of smart VLC networks-from data analysis to behavior prediction. In this work, we use kNN supervised learning technique, which is a simple and powerful algorithm intended to solve classification and clustering problems to combat the solar irradiance effect on V2V-VLC 
system. It is a straightforward and effective method to find and indicates associations within a given data set.

\subsubsection{System Model}

In this work, we consider the same model which is given by section 2.2 and shown in Figure. 6.2 to apply MLE for performance enhancement of VLC-based V2V systems under a sunlight effect. Moreover, we have chosen the city of Toronto as a reference location for consideration of solar irradiance during the summer season. We consider the solar irradiance listed in Table. 6.1, and OOK modulation scheme for VLC signal.

\subsubsection{Machine Learning and Adaptive Filtering}

We apply an MLE algorithm for the training of an optical filter. This filter is installed within the taillights (the receiver) of the receiving vehicle. This filter adaptively modifies itself responding to physical conditions of the VLC link-attempting to achieve an acceptable BER.

Depending upon the environmental conditions, the values for solar irradiance will change with weather and overtime day. The distance between two communicating vehicles will also vary due to the relative speeds between them. The FOV is expected to change with vehicle size and make, style and width of headlights and taillights. All of these factors effect the reception of the VLC signal and, in turn, effect the received BER.

We train our system using supervised learning. We use the Nearest Neighbour Algorithm, which belongs to "Supervised Learning" MLE class.

\subsubsection{Why kNN Algorithm}

$\mathrm{kNN}$ algorithm is one of the simplest classification algorithms and it is one of the most used learning algorithms. Also, $\mathrm{kNN}$ is a non-parametric, lazy learning algorithm. Its purpose is to use a database in which the data points are separated into several classes to predict the classification of a new sample point. 


\subsubsection{Advantages}

- Simple to implement

- Flexible to feature/distance choices

- Naturally handles multi-class cases

- Can do well in practice with enough representative data

\subsubsection{Disadvantages}

- Large search problem to find nearest neighbours

- Storage of data

- Must know we have a meaningful distance function

- The accuracy of kNN can be severely degraded with high-dimension data because there is little difference between the nearest and farthest neighbor.

In our research, we use $\mathrm{kNN}$ because we are dealing with two variables only (FOV, and solar irradiance) so we do not need too much storage data nor high dimension data, thus, the calculation time will be reasonable. Also, we suggest a weighed voting factor to remedy skewed class distributions.

\subsubsection{Problem Classification}

We train the system using the nearest neighbor supervised MLE algorithm. We use data for the city of Toronto for various values of solar irradiance which is shown in Table. 6.1. After developing the data set for various parameters affecting the VLC signal, the nearest neighbor algorithm was applied to find the closest match of the actual transmitted VLC signal. Our algorithm finds the closest neighbor to the transmitted signal and finds the effective BER. If this BER is over a certain threshold, our system modifies itself to achieve the desired acceptable rate. 


\subsubsection{Adaptive Filtering}

We don't give detailed of the filter design in this work and leave it for the future work. There are many factors that can effect BER (transmitted power, incident angle, received angle, distance, solar irradiance), but for now, we consider only two parameters, i.e, FOV, and solar irradiance. We train our system using $\mathrm{kNN}$, and it will adjust itself according to the difference between achieved and desired BER. The system can perform two actions, depending upon the received VLC signal and achieved BER.

- Blocking Solar Irradiance: We assume our filter is comprised of cascading films/blinds and can automatically make these films appear before APDs, for irradiance noise filtering from the VLC signal. The filter will adjust the number of films/blinds according to the intensity of solar irradiance. If the effect of solar irradiance is greater, the received BER is expected to be worse, therefore, the smart filter will automatically add more blinds to nullify the effect of ambient noise.

- Modifying the field of View Angle: FOV can affect the VLC signal, as the incident angle of the received signal changes with different inclinations of taillight mounts. Changes in the incident angle will change the received signal strength and effect the BER. If achieved BER is not acceptable, our system will modify the inclination angle of taillights for proper FOV to achieve a better BER. The sequence of operations for our algorithm is given in Figure. 6.11.

\subsection{6 k-Nearest Neighbour Algorithm and Distance weighted kNN rule}

$\mathrm{kNN}$, is a well-known algorithm used in the pattern recognition literature. According to this algorithm, an unclassified pattern (sample, instance) is assigned to the class represented by a majority of its K-nearest neighbours. This rule is usually called the voting kNN rule. The 


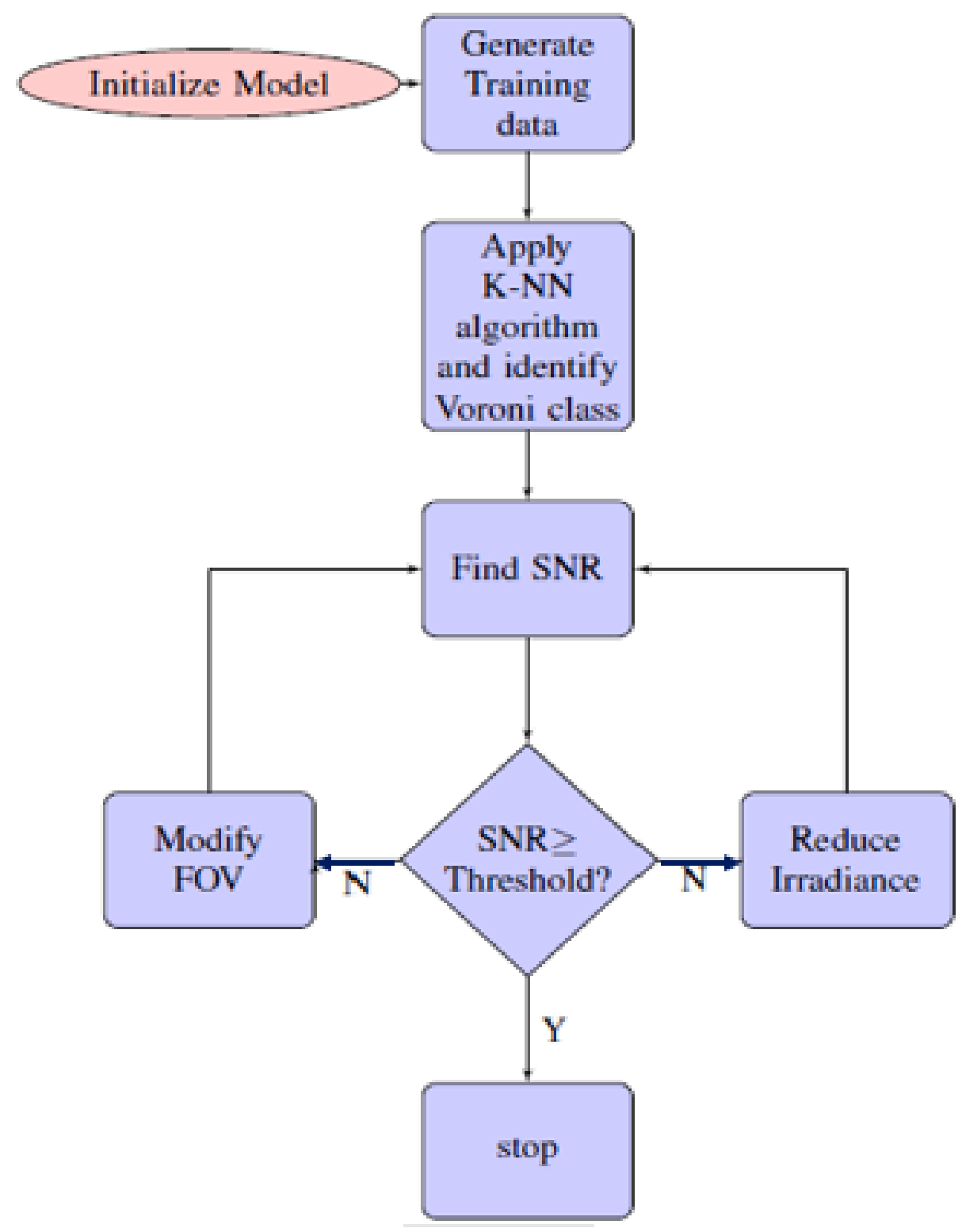

Figure 6.11: Adaptive filtering using kNN algorithm

number $\mathrm{N}$ of patterns and $\mathrm{k}$ are related such that, $K / N \mapsto 0$, the error rate of the kNN rule approaches the optimal Bayes error rate.

$\mathrm{kNN}$ regression is a nonparametric regression method, where the information derived from the observed data is applied to forecast the amount of predicted variable in real time without defining a predetermined parametric relation between predictor and predicted variables. Also, in voting $\mathrm{kNN}$, the $k$ neighbours are implicitly assumed to have equal weight in the decision, 
regardless of their distances to the observed data $x$ to be classified, or, otherwise, the weight should be define for each neighbour. The basis of this method is on calculating the similarity (neighborhood) of the real-time number of predictors $X_{r}=x_{1 r}, x_{2 r}, x_{3 r}, x_{m r}$ with the amount of predictors for each historical observations $X_{t}=x_{1 t}, x_{2 t}, x_{3 t}, x_{m t}$ via Euclidean distance function $D_{E c}$ or any other distance method. In this work, we use two methods, Euclidian distance and City block method (Manhattan distance) in seek of comparison the performance.

Euclidian distance is given as follows

$$
D_{E c}=\sqrt{\sum_{i=1}^{m} w_{i}\left(x_{i r}-x_{i t}\right)^{2}} \quad t=1,2,3, \ldots, n
$$

where $w_{i}(i=1,2, \ldots, m)$ are the weights of the predictors, summation of which is equal to one.

The City block distance between two points is calculated as

$$
E_{b o}=\sum_{i=1}^{K}\left|\left(x_{i r}-x_{i t}\right)\right|
$$

In this work, the weight $w_{i}$ is calculated as follows

$$
w_{i}= \begin{cases}\frac{d\left(x_{k}, x\right)-d\left(x_{i}, x\right)}{d\left(x_{k}, x\right)-d\left(x_{1}, x\right)} & \text { if } \quad d\left(x_{k}, x\right) \neq d\left(x_{1}, x\right) \\ 1 & \text { if } \quad d\left(x_{k}, x\right)=d\left(x_{1}, x\right)\end{cases}
$$

where $d\left(x_{k}, x\right)$ is the distance between the $k^{t h}$ neighbor and the observation $x$.

More formally, given a positive integer $\mathrm{K}$, an unseen observation $x$ and a similarity metric distance, kNN classifier performs the following two steps:

Step A : Distance between Data Points For certain value of K, the distance between observed data and its neighbors can be calculated using different methods such as Minkowsky, Euclidian, Cityblock, etc... Afterward, measured distances are sorted to determine the nearest 
neighbor based on the $k^{t h}$ minimum distance. Thereafter, the categories of the nearest neighbors are gathered to do the voting. The algorithm runs through the entire data set, computing the distance $D$ between $x$ and each training data element. $\mathrm{K}$ points in training data closest to $x$ are saved in set A.

Step B : Categorial Assignment For an instance x (observed data), assume we have $N_{i}$ instances belong to class $Y_{i}$ in the neighborhood. Then we define

$$
P\left(Y_{i} / x\right)=\frac{N_{i}+s}{K+C s}
$$

where $K$ is the total number of instances in the neighborhood, $\mathrm{C}$ is the total number of classes, and $\mathrm{s}$ is the smoothing parameter. The smoothing is used to avoid 0 probabilities.

A detailed self-explanatory description of our proposed algorithm is given in algorithm 1 .

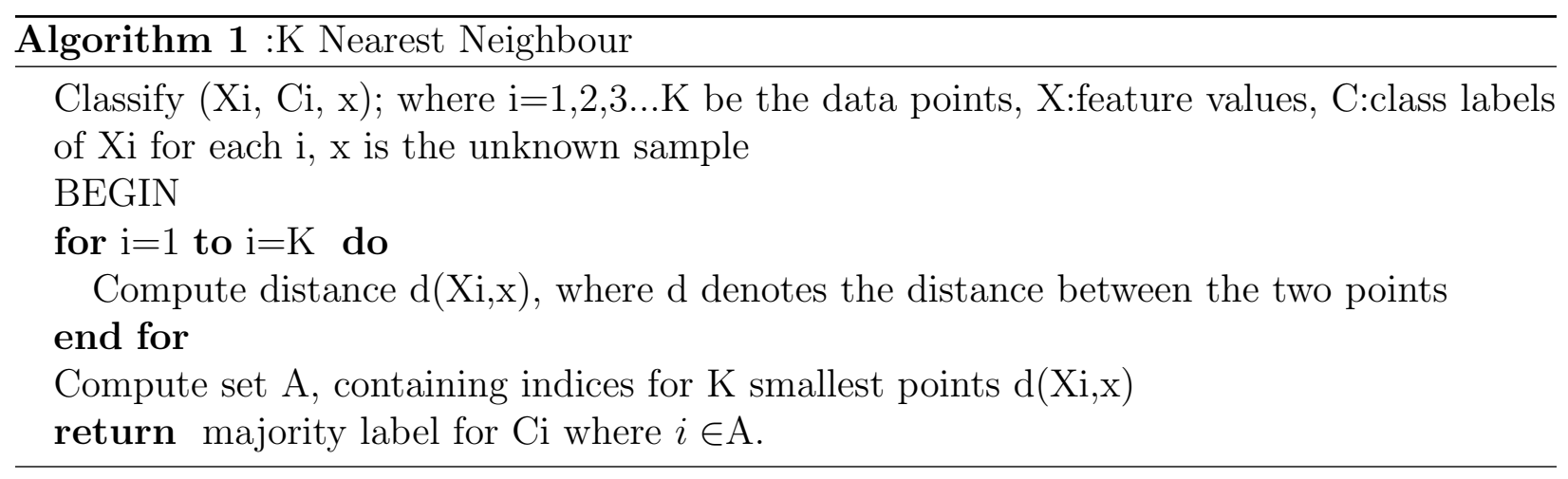

\subsubsection{Simulation Results}

In the simulation, we use the MLE toolbox in the Matlab suit of software. The simulation parameters are shown in Tables. 1.2, 2.1, 3.1, and 6.1.

\subsubsection{Data Sets and Classes}

In Figure. 6.12, the Voronoi diagram is shown to represent the training and the observed data and their distribution to various classes on the basis of calculated BER. We plot data 


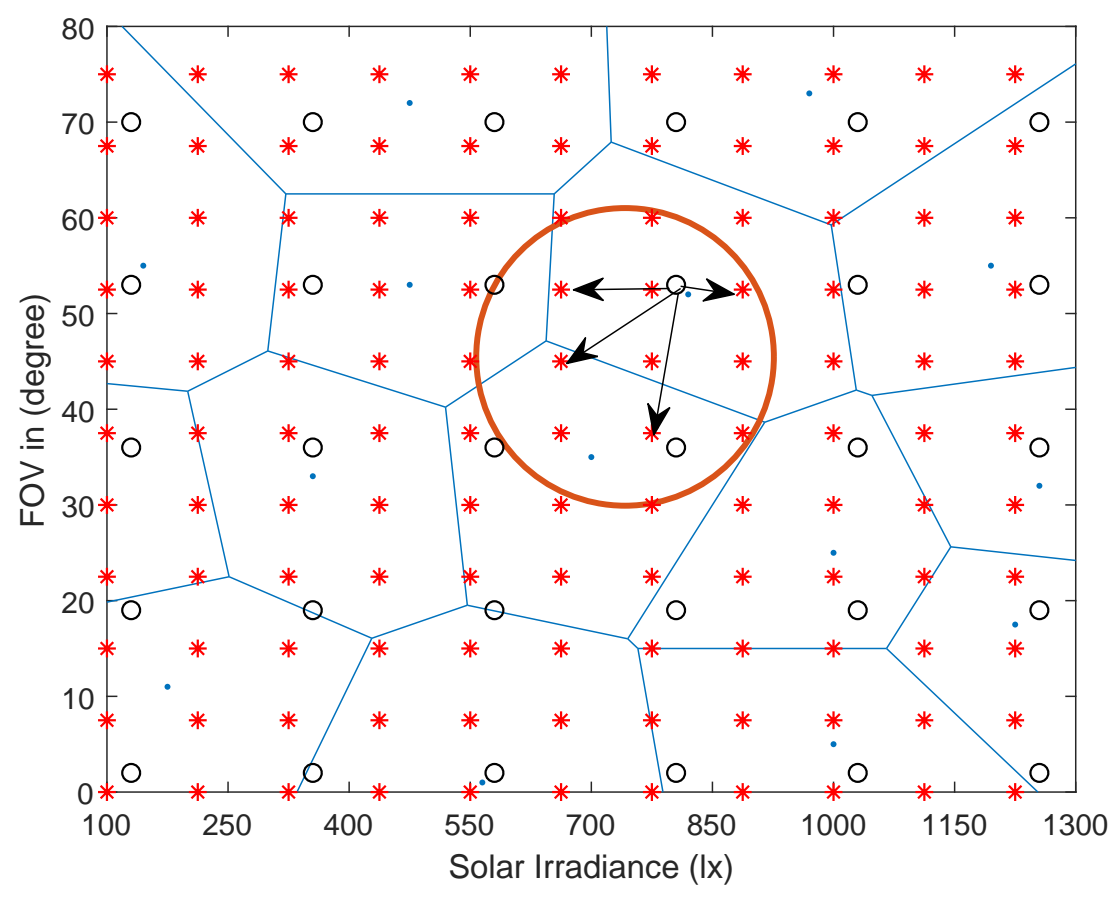

Figure 6.12: Training data and observed data in Voronoi diagram.

sets as a Voronoi diagram to make it easy to recognize the boundaries of decisions areas. The classification of a data sets is based on the classes of its nearest neighbours. Since each data set consists of two variables; solar irradiance, and FOV, the x-axis represents the irradiance and the y axis represents the FOV.

The functionality of $\mathrm{kNN}$ algorithm can be explained by considering one element of the data set. We denote the observed data in Voronoi, with a small circle'o', and the training data by small '*', for a certain observed data point. For a certain value of $\mathrm{k}$, the distance between observed data and its neighbors can be calculated. Afterward, measured distances are sorted to determine the nearest neighbor based on the $k^{\text {th }}$ minimum distance.

\subsubsection{Choice of $\mathrm{k}$}

$\mathrm{K}$ in $\mathrm{kNN}$ is the number of instances that we take into account for the determination of affinity with classes. So we need to investigate the performance of kNN near rule-of-thumb-value and make a decision about the optimal one using any algorithm for performance testing. 
In Figure. 6.13, the effect of the numbers of selected neighbors on the decision accuracy is presented. We use the Euclidian distance formula and City block distance formula to generate this figure, and use a Monte Carlo method to average it over 2000 iterations. It is shown that optimal value of $\mathrm{K}$ is 12 with Euclidian distance and $\mathrm{K}$ is 6 with City block distance.

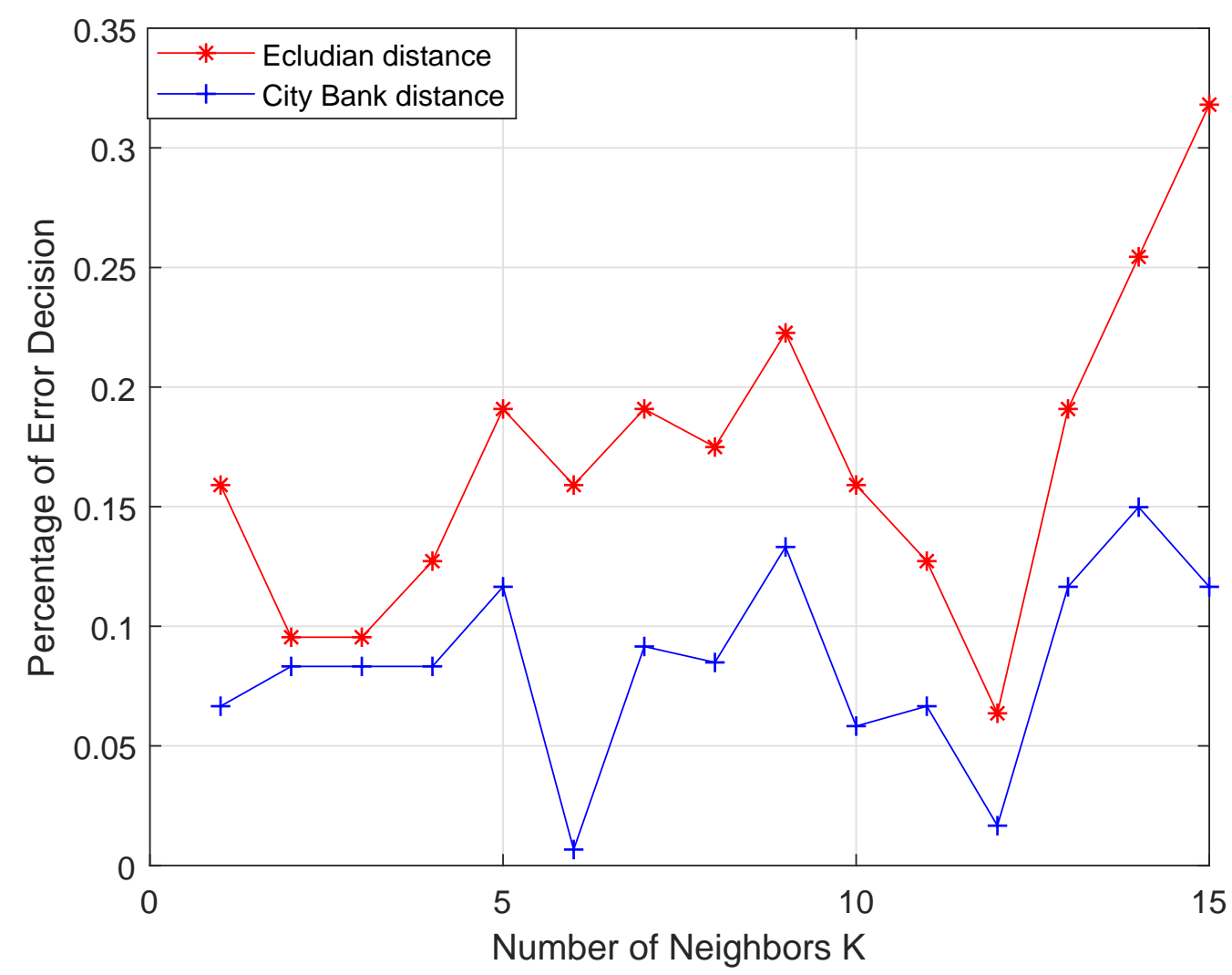

Figure 6.13: The relation between the number of neighbors and the percentage of decision error.

\subsubsection{BER Discussion}

Figure. 6.14 represents the BER of the proposed system before applying the denoising scheme and after applying the scheme. Figure. 6.14 shows improvement in terms of BER after applying the denoising scheme. We can notice an improvement in BER from $10^{-2}$ to $10^{-5}$, thus, reflecting the stability and quality of the communication link.

Figure. 6.15 shows the probability of error of each action (add optical filter or change the 
FOV) at each daytime hours. Because choosing the action is randomly, this result may help to determine which action of the system has to be chosen at each hour of the day. Moreover, Figure. 6.15 shows the probability of adding optical filter is higher than changing the FOV, the reason is that while times change, the position of the sun also changes and the Irradiance value will change.

\subsubsection{Execution Time}

In Figure. 6.16, we discuss the execution time for the proposed scheme. We consider two methods to calculate the distance between the instance and its neighbors, Euclidian distance, and City block distance. Also, we use Intel(R) Core (TM) i5-5200U CPU@ 2.20 GHz laptop to calculate the execution time for the data sets. Figure. 6.16 shows both methods almost have the same time of execution for a low numbers of data sets 0.05 sec for 5000 data set, while Cityblock method is better for a high number of data sets $0.32 \mathrm{sec}$ for 50000 data set.

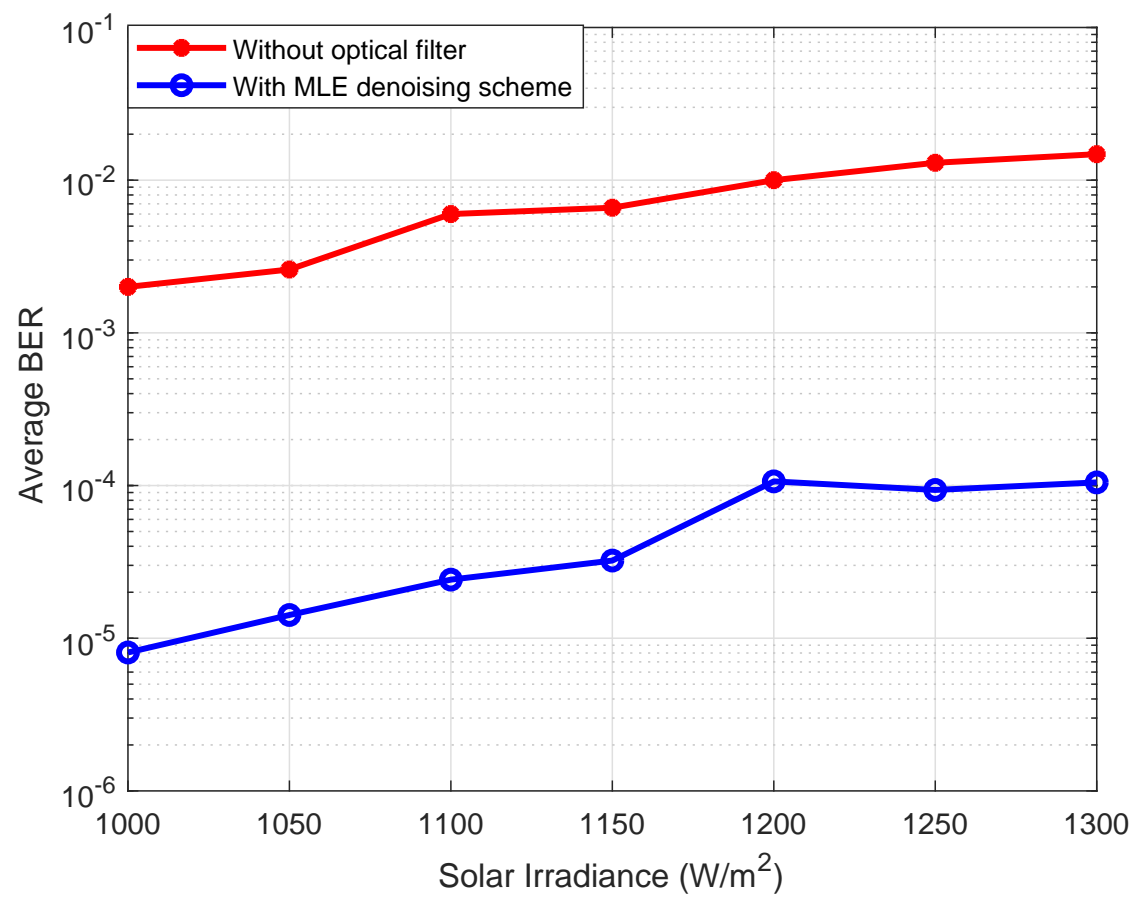

Figure 6.14: BER of the V2V-VLC system with and without denoising scheme. 


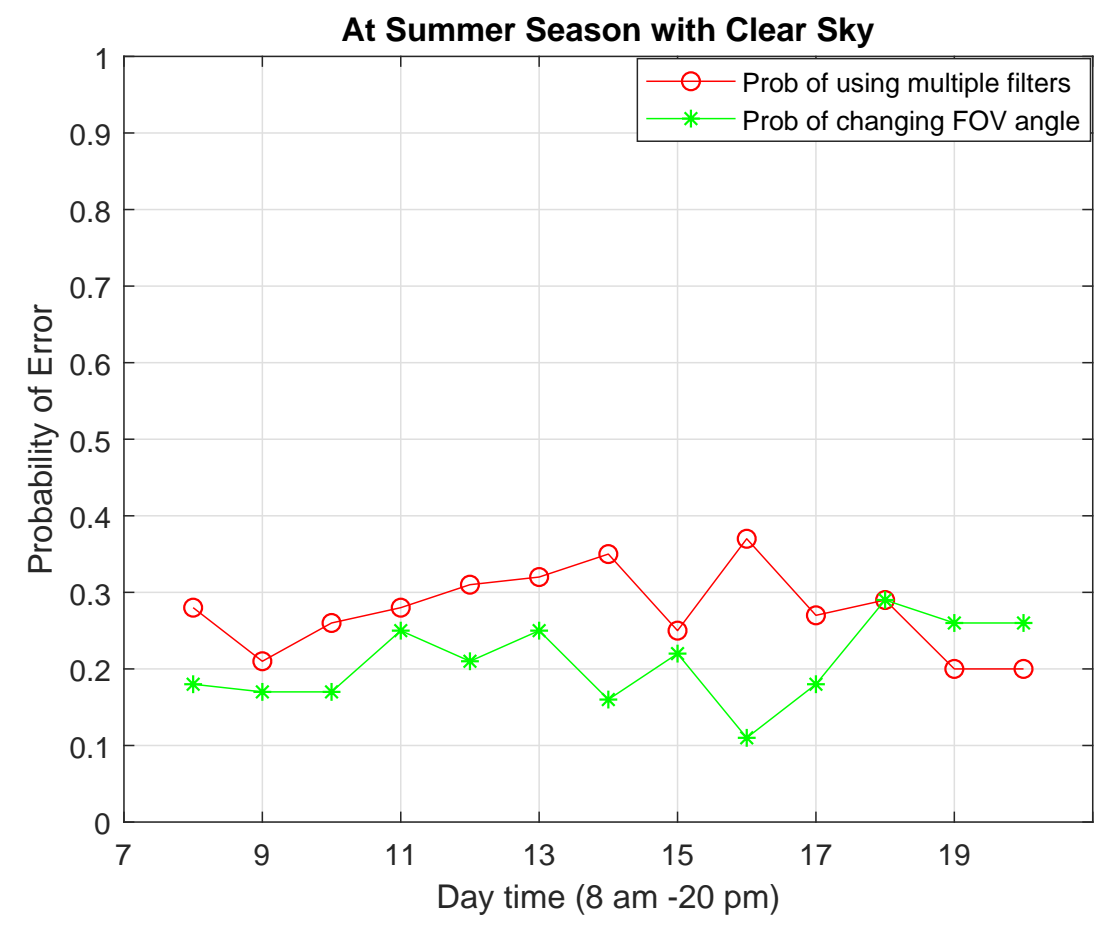

Figure 6.15: Probability of each action with day hours.

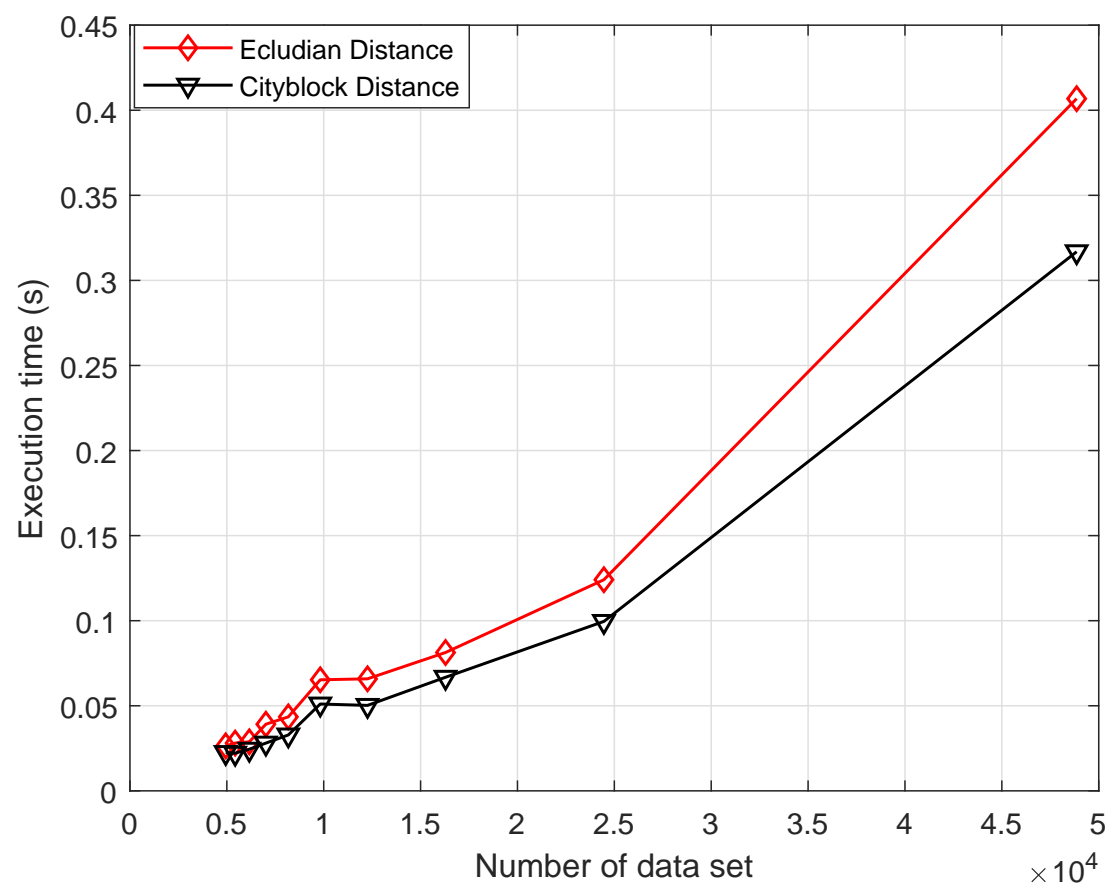

Figure 6.16: Execution time for the proposed scheme. 


\subsection{Chapter Summary}

This chapter proposes two denoising schemes to combat the effect of solar irradiance, thus, improve the performance of V2V-VLC systems under sunlight effect. The first proposed denoising scheme is by using a differential receiver, while the second proposed scheme is by using a kNN algorithm as machine learning based adaptive filter. Simulation results of both schemes show good improvement in SNR, which make the communication link more stable even in months of summer. 


\section{Chapter 7}

\section{Applications on the Proposed Channel Model}

Because VLC systems have a dual purpose of illumination and communication, we get this advantage to present an important application in ITS field using the channel model given in chapter 2. In this chapter, we present a reporting architecture for emergency situations and, management through Foglets and VLC as a part of ITS. We propose the use of Foglets near roadside to improve information processing and Quality of Service (QoS) and to reduce communication delay. We use the VLC link between Foglets and smart vehicles and discuss its safety and suitability for the road assets infrastructure, as compared to conventional RF links. We model this VLC link and discuss its luminosity and BER characteristics.

\subsection{VLC Enabled Foglets Assisted Road Asset Reporting}

There has been a lot of work on emergency reporting in smart transportation systems, but we find less information about roadside asset reporting and management. Currently, available mechanisms do not efficiently handle asset management and associated emergency reporting. Asset management is based on either reactive maintenance (reported by people) or preventative scheduled maintenance (scheduled). In this work, we present a reporting 
architecture for emergency situations and management through Foglets and VLC as an important part of ITS. We propose the use of Foglets near roadside to improve information processing and QoS and to reduce communication delay. We use the VLC link between Foglets and smart vehicles and discuss its safety and suitability for the road assets infrastructure, as compared to conventional RF links. We model this VLC link and discuss its luminosity and BER characteristics. We emphasize that how Fog computing will enable the road asset management and hazard reporting by utilizing the smart vehicles. It has a wide application spectrum as it will support public security, infrastructure management, and emergency medical aid.

\subsection{VLC Enabled Foglets}

New applications, such as augmented reality techniques, self-driving, smart vehicles and transportation etc.; all deal with complex data processing, and storing operations. It requires a higher level of data communication, computation, and storage capabilities.

Communication capacity is of great significance in smart transportation infrastructure as information exchanges cannot happen without reliable communications. To deal with the higher level of data communication, VLC has proved itself to be a promising technique in terms of data rate and negligible electromagnetic interference.

In order to deal with the computation and storage requirement, Fog computing is a promising computing platform [105]. It is based on distributed computing and deployments in contrast to the cloud; which is centralized. Since it is localized, it provides low-latency communication and more context awareness.

We combine the best of two worlds to provide instant asset and incident reporting. We benefit from the high data rates of VLC and greater computational speed of Foglets to report for the hit and run cases and asset damage reporting. We use smart vehicles and employ them as infrastructure, and moving features of vehicles carry the information from one place to another place. Moving vehicles become good message carriers and can continuously 
transmit information by building up new connections with accessible Foglets. Instead of sending information to the cloud servers, damage reporting tasks are completed by utilizing computational and communication resources locally inside Foglets. All these bring less delay, lower power and lower cost of operation.

\subsubsection{Fog Computing}

Fog computing is used to enable applications on billions of connected devices, already connected in the Internet of Things (IoT), to run directly at the network edge [106]. Both the cloud and the fog provide data, compute, storage, and application services to end-users. The distinguishing fog characteristics are its proximity to end users, its dense geographical distribution, and its support for mobility in Fog networks (FogNets). The main characteristics of FogNet include ubiquity, de-centralized management, and cooperation [107]. A large amount of devices are connected to the internet in these FogNets. These devices form many "mini clouds" at the edge of the network and manage themselves in a distributed way. FogNet, therefore, may significantly alleviate the computing and routing burdens in the cloud-part of networks to achieve the scalability. The users need not download the data from the core network, instead, they just download the required data from their neighbors, and hence reduction of end-to-end latency is obtained. The Fog vision is conceived to address applications and services that do not fit well in the paradigm of the cloud [108]. It includes but not limited to:

- Applications that require very low and predictable latency such as gaming and video conferences. Fog frees the user from precise knowledge of where the computation and storage take place.

- Geo-distributed applications (pipeline monitoring, sensor networks to monitor the environment).

- Fast mobile applications (smartly connected vehicle, connected rail). 
- Large-scale distributed control systems (smart grid, connected rail, smart traffic light systems).

The Fog computing paradigm is well positioned for real-time big data analytics, supports densely distributed data collection points, and provides advantages in entertainment, advertising, personal computing and other applications. Fog computing based architectures can be roughly modeled by a simple three-level hierarchy, where each smart thing is attached to one of Fog devices, Fog devices could be interconnected, and each of them is linked to the cloud.

\subsubsection{Challenges and Motivation}

To solve the communication and computational capacity problem for smart vehicles and latency sensitive application, existing methods include $3 \mathrm{G}$ and $4 \mathrm{G}$ cellular networks, RSU, and mobile cloud computing. However, these are not sufficient as cellular networks provide supportive communication but are controlled primarily by network operators. This is not efficient or particularly effective from the application perspective. RSUs enlarge the network communication capacity but are expensive and are not applicable for delay sensitive applications.

As high quality of network connections are required when uploading real-time information otherwise it will be time consuming to exchange information from remote servers. Mobile cloud computing can bring rich computational resources to mobile users but is costly and time consuming. Therefore, it remains a great challenge for researchers and engineers to deal with communication and computational demands efficiently and conveniently.

\subsubsection{Foglets and Distributed Data Processing}

Most of IoT applications and specifically our presented infrastructure requires processing

closer to the road, and a tighter coupling between events and actions. We might introduce unnecessary latency and greater potential for network failure, by utilizing the centralized cloud for events requiring immediate actions. We propose to bring computing and storage of 
network resources near the smart vehicles and roads. It will improve efficiency and reducing bandwidth requirement and latency, however, coordination between resources and devices for distributed processing is challenging.

\subsubsection{Why VLC for Foglets}

VLC technology has many essentials advantages over the RF based devoted short range communication technology, which could be adopted for ITS applications. Offering low complexity and a low cost, particularly in places where LED lamps are already installed in vehicles, traffic lights and street lights are considering as a first advantage, while high precision positioning, owing to the high directional LOS propagation characteristics be a second advantage. The VLC based positioning technology is able to reduce the positioning error to tens of centimetres, which is more accurate than the RF based positioning technology. One more advantage is the scalability, where the high scalability [109], as vehicle density increases, e.g. during rush hours, traditional RF will typically experience undesirable packet collisions and longer delays as well as poor packet reception rate. However, integration of VLC technology with ITS faces two important challenges, the metrological issues like (rain, fog, dust, etc.) and the background noise which will be reflected on the maximum data rate that can be transmitted.

\subsection{Road Asset Management and Incident Reporting}

We propose road asset reporting with the help of smart vehicles and Foglets as shown in Figure 7.1. Foglets are true enabler for services requires low latency such as connected vehicles and accident reporting. Smart vehicles will interact with roadside Foglets and also with

other smart vehicles. As these vehicles are smart vehicles, we assume that they have unique identification or IP address. Communication link between smart vehicles and Foglets is VLC. Headlights and taillights are used as transmitters for information transfer, while camera in Foglets are the receivers of this transmitted information. Vehicle lights have started to utilize 


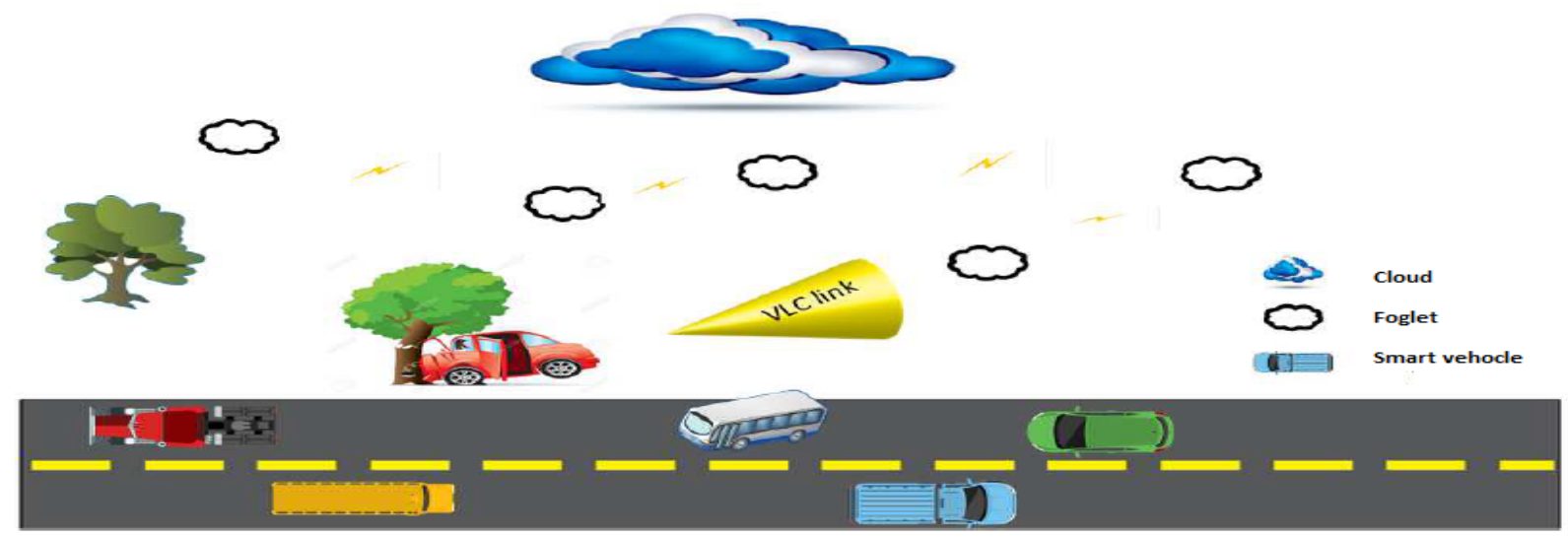

Figure 7.1: VLC Assisted asset management infrastructure.

LEDs and due to their shorter response time, they can be easily modified to become VLC transmitters.

We have the following goals to be achieved:

- Accident reporting and hit and run cars identifications.

- Maintenance and incident reporting of road side asset like traffic lights, water reservoir, trees etc.

- Collection of relevant data to evaluate and improve the system.

We want to divide theses tasks between Foglet and the centralized cloud. Tasks which are not handled well by Fogs are assigned to clouds.

\subsubsection{Infrastructure}

Proposed infrastructure is shown in Figure 7.1. We require Foglets to be installed at roadside at certain distances. We are not investigating the placement of these Foglets rather we assume that these optimally placed and serve the wide area under observation. We suggest their placement at barren places where there is no other reporting mechanism for potential disaster. We have smart vehicles which can communicate with Foglets and which in turns 


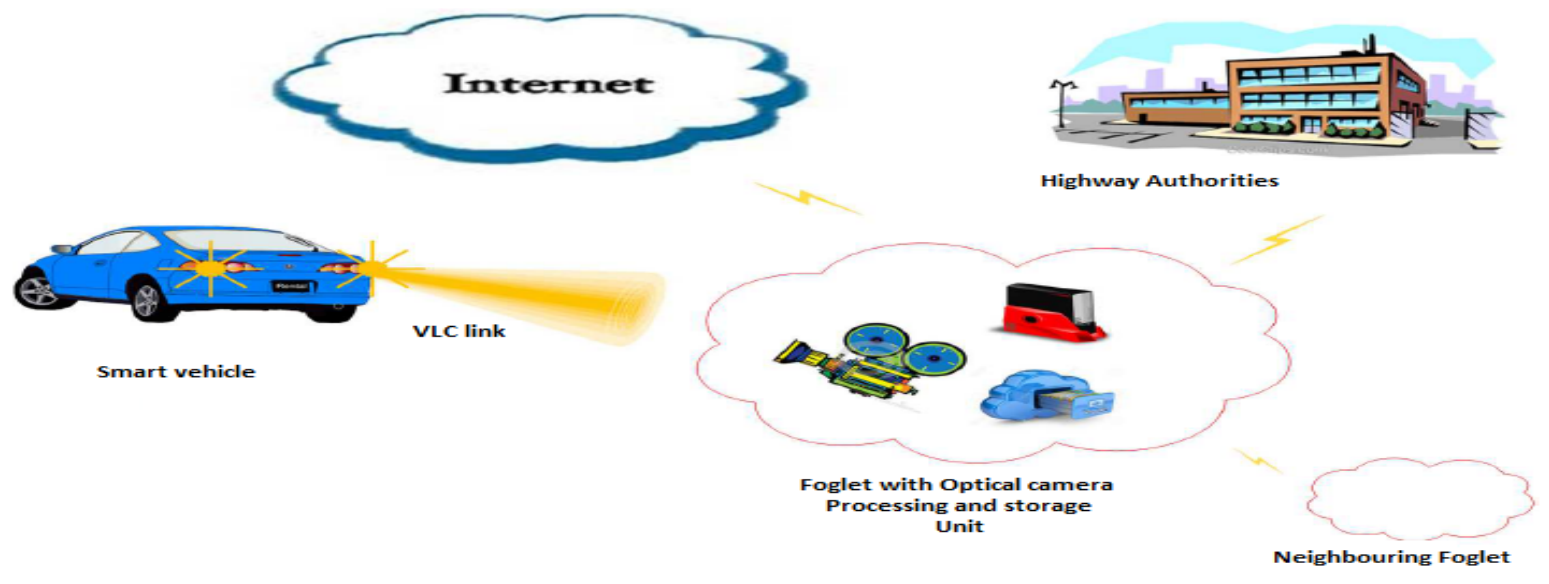

Figure 7.2: Foglets architecture.

are in communication with the centralized cloud. These Foglets are also in communication with each other thus creating an interconnected network of distributed nodes.

\subsubsection{Smart Vehicle}

Smart vehicles, through their advanced communication capabilities, are able to interact not only with navigation and broadcast satellites, but also with passenger smart phones, roadside units and other smart vehicles.

\subsubsection{Foglets Infrastructure}

Detailed Foglet architecture is shown in Figure 7.2. Foglets are comprised of cameras with special optical sensors. These Foglets are connected to one another and to centralized cloud through cellular connectivity.

\subsubsection{Rolling Shutter Cameras}

Cameras have been used for safety and comfort applications in the automotive field [33]. Therefore, using the camera as the optical signal receiver is reasonable and straightforward. Moreover, the camera receiver provides the non-interference communication capability due to the excellent spatial separation capability of the image sensor mounted in the camera. 
Therefore, non-crosstalk communication with multi-LEDs without a complicated protocol and processing is achieved by using a camera (image sensor). It also prevents optical signals from being mixed with noise (incident sunlight) and enables simple link designs.

Rolling shutter is a method of image acquisition in which each frame is recorded not from a snapshot obtained at a single point of time, but rather by scanning across either vertically or horizontally [110]. The transmitting LED light switches on and off at very high frequencies according to the modulation, and the pixels of the camera sensor activates sequentially (by row). Therefore does not get the entire image simultaneously. When the rows of pixels are activated, they are exposed to the light at that time and then their values are stored. After the procedure is completed, the scan lines captured at different time are merged together to form a single image.

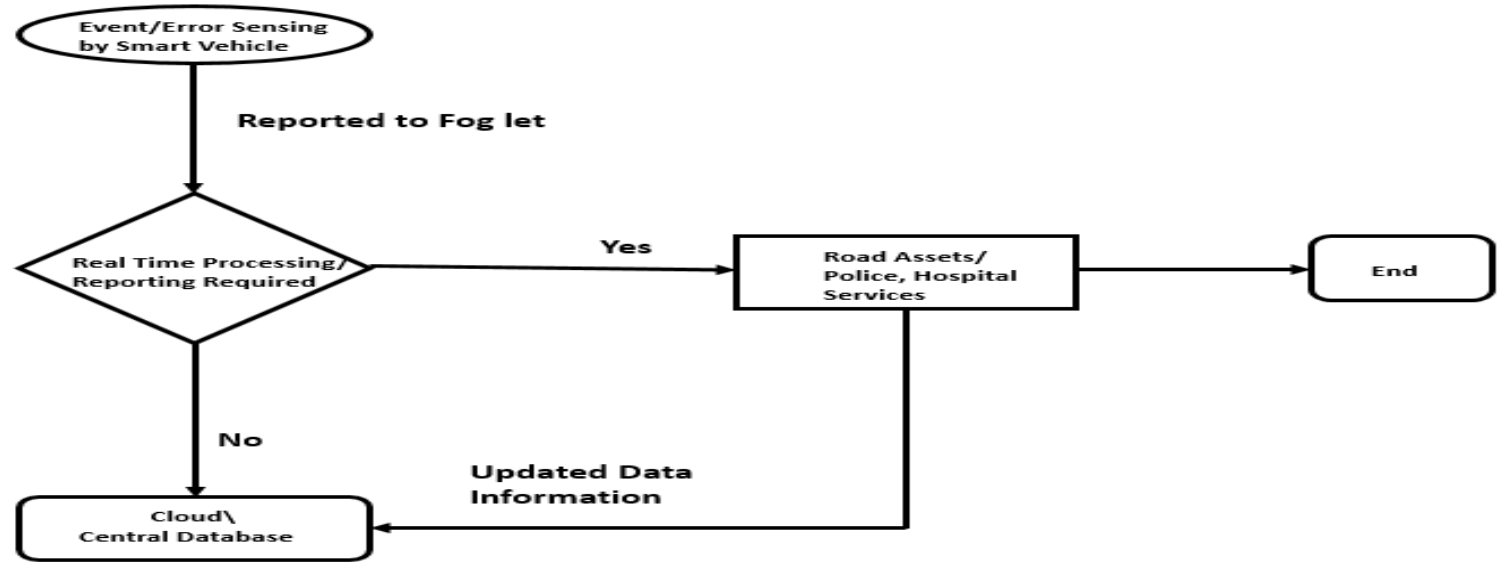

Figure 7.3: Sequence of Foglets tasks

\subsubsection{Optical Communication Sensors}

When we use cameras to capture images from vehicles, achieved data rate and accurate and quick LED detection are the main concerns. The data rates per pixel of typical camera receivers are in the tens of $\mathrm{kb} / \mathrm{s}$ or less. However, for transmitting multi-media data from high speed vehicle, higher data rates such as few $\mathrm{Mb} / \mathrm{s} /$ pixel are required. Additionally, the receiver system has to find the LED transmitters in captured images via image processing 
techniques. However, it is very hard to correctly and promptly detect LED transmitters from images under outdoor lighting environments with a low computing cost. Therefore, we purpose Optical communication image sensors [32] to be used in the cameras mounted in Foglets. These sensors have a non-conventional pixel, a communication pixel, which is specialized for high-speed optical signal reception. Additionally, it has an output circuit for a non-conventional image, which only reacts to high-intensity light sources such as LEDs and thus facilitates the LED detection. It is specially designed for receptions of high-speed optical signals. It provides substantially improved response speed to light intensity variations and has $20 \mathrm{Mb} / \mathrm{s}$ optical signal reception per pixel.

\subsubsection{Foglets Reporting}

Traffic and hazard information from fog devices is forwarded to the cloud for global coordination. Fog devices are decision makers and coordinate locally with neighboring fog devices and smart vehicles on the roads. If we elaborate further on our target goals (as listed earlier), we can see that all of them has different requirements in terms of time, processing and need. The first goal of accident reporting and hit and run cases require real time processing. The second objective of Asset management requires real time reporting and also global analysis and management. The third objective relates to the collection and analysis of global data over long periods. We assign data accusation and processing of first two tasks to Foglets and third to cloud. Figure 7.3 shows the sequence of tasks performed by our proposed system.

\subsection{Investigate VLC Channel between Vehicle and Foglets}

In this section, suggested VLC channel is presented. In our model, we consider the vehicle as a transmitter and the Foglet as a receiver for VLC link. We consider a number of cars passing through the road and the road surface act as a reflector. a BRDF is used to model 
the reflectors in this work. Optical wireless channel involved noise can be modelled as[52]

$$
y(t)=\gamma x(t) \otimes h(t)+n(t)
$$

where $x(t)$ denotes the transmitted signal and $y(t)$ represents the received signals, respectively, $\gamma$ denotes the conversion efficiency between optical and electrical signals, $h(t)$ denotes the channel impulse response, $\otimes$ means convolution and $n(t)$ denotes the Gaussian noise. In the proposed model, there are two paths for the light, the LOS between the smart vehicle and the Foglet unit, and the NLOS path between the smart vehicle and the Foglet unit through any reflector. The channels gain of LOS is given by (4.11), and the NLOS path transfer function through any reflector is given by (4.12), (4.13), and (4.14).

\subsubsection{BER of VLC Link}

To study the performance of our proposed system, we need to find the BER and inturns the noise power. The shot noise induced by the solar radiation remains the main source of noise for VLC links during the daytime, as the artificial light induced interference has lower intensity than the solar radiation. However, the interference due to the artificial lights is the dominant noise source during the night time [111]. In our proposed system, we assumed OOK modulation and a Gaussian noise having a total variance that is the sum of contributions from the shot noise and thermal noise association with the receiver.

\subsubsection{Results and Discussion}

We used Matlab software and realize parameters to model the VLC channel for the proposed system. Before we perform the comparative analysis of VLC and RF link between Foglets and smart vehicles, we try to find the optimum positioning of Foglets to obtain better reception and BER. For this purpose, we find the luminous intensity of head/tail lamps for various distances from the road. In Figure. 7.4, the isocandela and isoilluminance diagrams of the 
road surface from a pair of high-beam headlamps with luminous intensities at $1500 \mathrm{~cd}$, are shown. It is apparent that for high beam headlamps, a narrow and flat beam is projected in a horizontal direction a few degrees to the left, providing a symmetrical illumination pattern on the road. However, the high-beam headlamps provide a symmetrical pattern designed to offer adequate forward and lateral illumination. This way, we can predict proper location to position the Foglet station to be within the headlight boundaries. Which in turns increase the optical power reception by improving the LOS path.

Although VLC link is less susceptible to multipath fading, we still want to consider direct and reflected path for accurate calculation of received optical power. In Figure 7.5 total received power is plotted versus the communication distance between Foglets and head/tail lamps. Also, we plot the LOS path power in addition to, the NLOS path power considering first order reflection system. Also, from Figure. 7.4 and Figure. 7.5, we notice that the good distance for communication between the vehicle and the Foglet station is up to $80-100 \mathrm{~m}$ when using the low-beam headlamps and about 120-150 m when using high-beam headlamp, where the illuminance will be about $1000(\mathrm{~cd})$.

Figure 7.6 shows the BER for the VLC link as a function of the LED illuminance intensity. It is evident that as the illuminance intensity increases the BER increases which help in design the distance between the transmitter and receiver. 


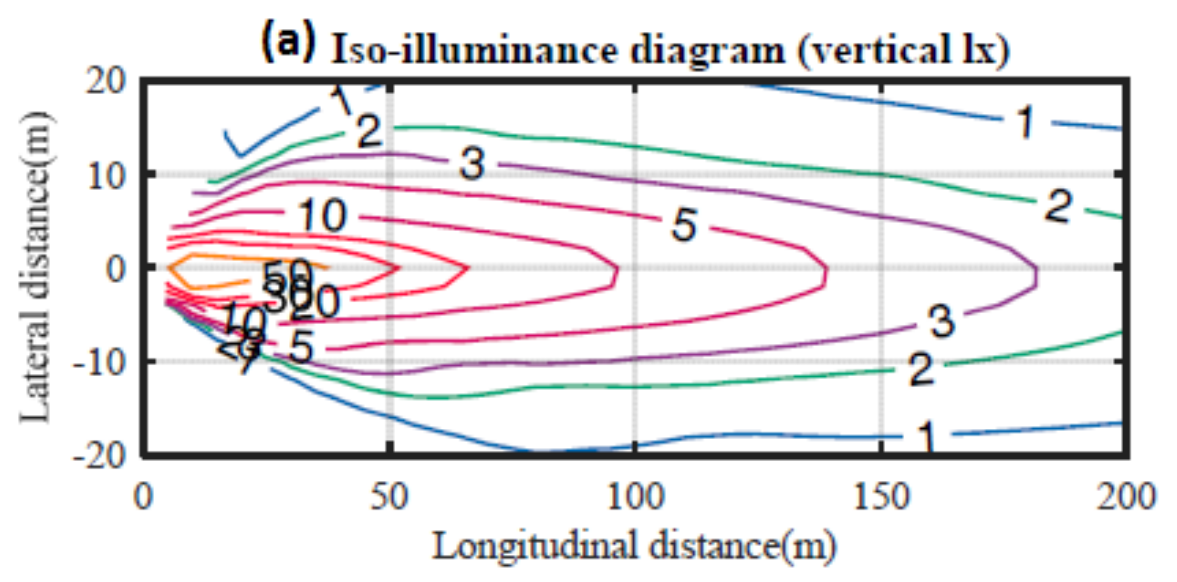

1003001000300010000

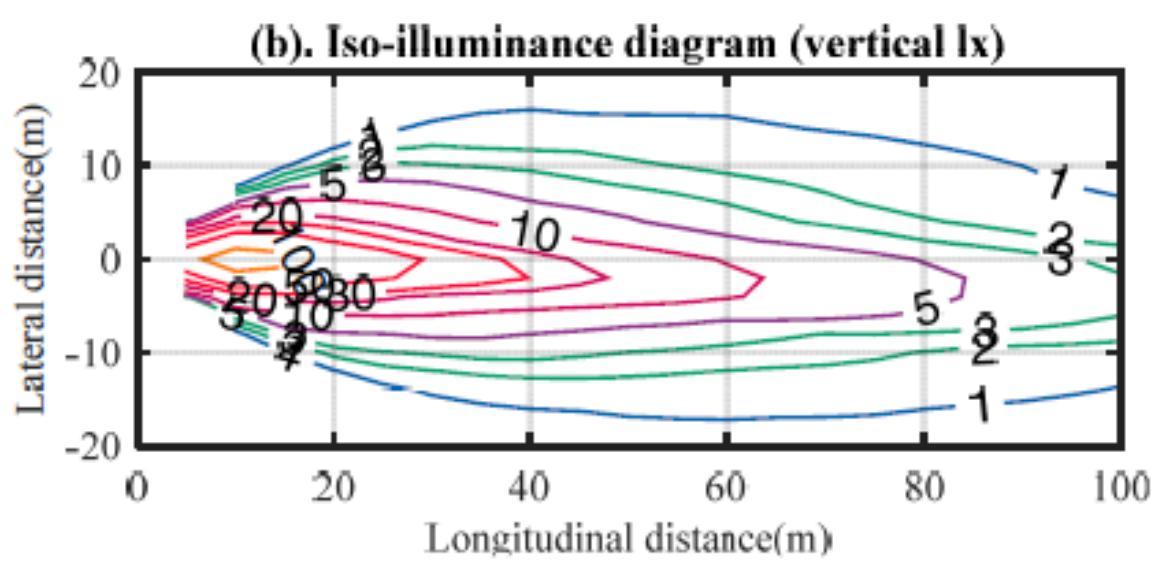

Figure 7.4: Isocandela diagrams of the road surface from a pair of low beam and high beam headlamp [7]. 


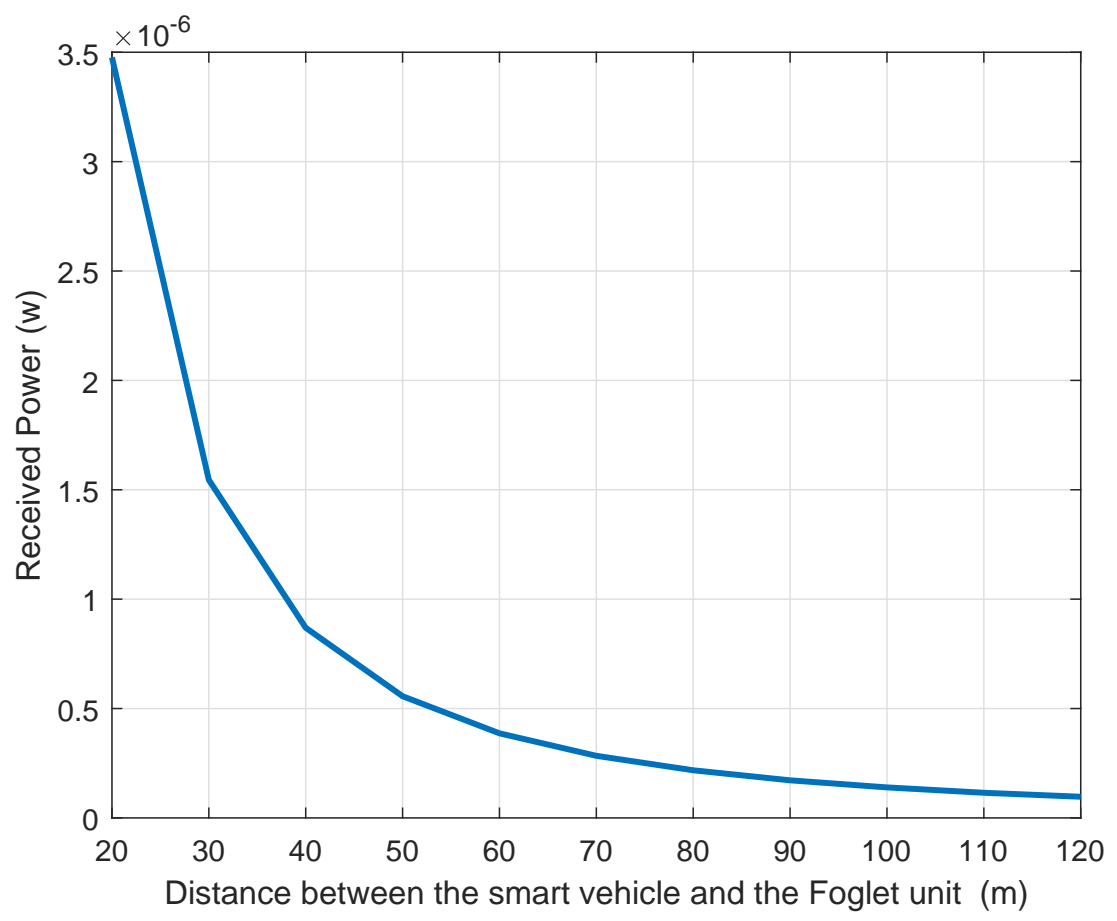

Figure 7.5: Received power vs the distance between the vehicle and the Foglet station.

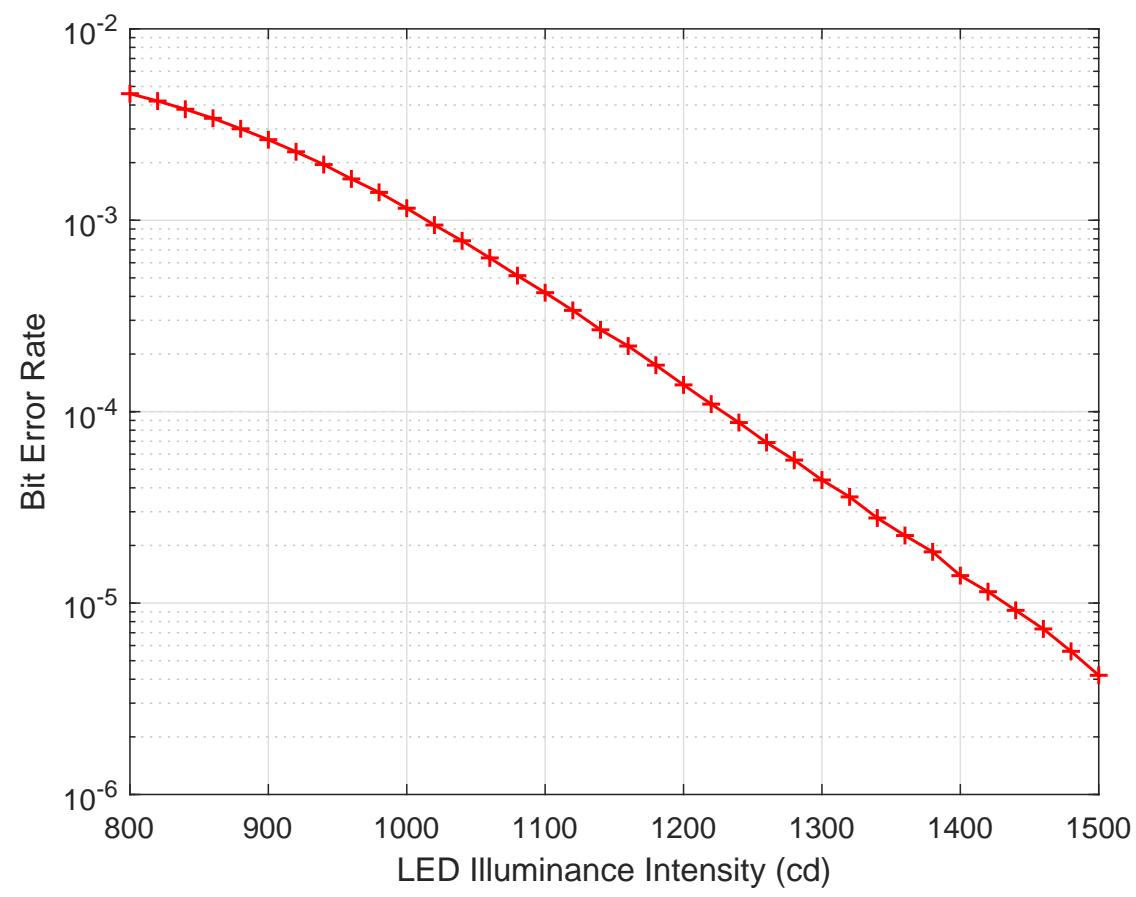

Figure 7.6: The BER vs the LED illuminance intensity 


\subsection{Chapter Summary}

In this chapter, we test the VLC channel model with another ITS application. we present a road asset reporting with the help of smart vehicles and Foglets visible light based to be a part of ITS. The performance of the system is investigated in term of BER and received power. 


\section{Chapter 8}

\section{Discussion and Future Work}

In this thesis, an efficient V2V-VLC system was studied, investigated, and evaluated by taking the following steps:

- We investigated the V2V communication system as a $2 \times 2 \mathrm{MIMO}$, taking into consideration the real-time environment, practical reflectors, and real ambient noise.

- We simulated the channel of communication and derived the CIR expressions for LOS path and NLOS path. Furthermore, we calculated many channel parameters such as: BER, SNR, RMS delay spread, coherence bandwidth, and coherence time when the vehicles move in the same direction and when they move in opposite direction.

- The O-OFDM was introduced to the V2V-VLC system. We proposed adaptive modulation schemes using DCO-OFDM and ACO-OFDM to combat ISI to improve the performance in terms of SNR, BER, and data rate.

- We investigated different precoding and equalizing schemes for the V2V-VLC system considering flickering/dimming control and the nature of the outdoor VLC channel as a frequency selective channel to enhance the system's performance.

- Because shadowing is an important issue in VLC systems and it could deteriorate the communication link, we studied the effect of the shadowing on the V2V-VLC system 
and we proposed the solution.

- To reduce the effect of sunlight on the outdoor VLC channel, we proposed two denoising schemes: the differential receiver with optical filter scheme, and kNN- MLE algorithm scheme.

- We simulated the VLC channel with another application of ITS by proposing a road asset reporting with the help of smart vehicles and Foglets visible light based.

All these contributions were preceded by a deep analysis of the existing VLC literature. This allowed the identification of the advantages, weaknesses, trends, and challenges related to the VLC development.

\subsection{Conclusions}

In this thesis, the main conclusions are:

- We studied a $2 \times 2$ MIMO channel model for V2V-VLC system considering LOS path and NLOS path. We introduced a new mathematical formula for the transfer function of the channel using the multiplication of matrices and calculated the channel impulse response using IFFT, where we prove that calculating infinite number of reflections are not needed. We simulated the V2V-VLC CIR of the LOS and the NLOS components and found the CIR of the LOS path is represented by an impulse while the CIR of the NLOS components has an exponential decay behavior. The simulation results showed that the gain of the LOS path is about $1.5 \times 10^{-6}$, and that of NLOS path is about $0.8 \times 10^{-6}$. The low gain for CIR for both paths is due to the nature of V2V-VLC system where the channel is changing very frequently.

The CIR is then used to find many channel parameters such as SNR, BER, RMS delay spread, coherence time, and coherence bandwidth. The simulation result showed the 
received power is inversely proportional with the distance between the transmitter and the receiver and it varies between $(-26 \mathrm{dBm}$ to $-18 \mathrm{dBm})$. Also, BER showed a good improvement compared with [7] because we consider MIMO link and we consider more practical reflectors in our work. Moreover, the PDF of the delay spread was found to be similar to Rician distribution with a mean value of $12 \mathrm{~ns}$, while the authors in [16] found it to be 10-15 ns. Moreover, we compared our results with that in literature as shown in Table. 2.2. Also, we found a 50\% and $90 \%$ coherence time of the suggested channel when the transmitter and the receiver are moving in the same direction and in the opposite direction, respectively. Hence, there is a decreasing trend in coherence time with effective speed for both directions, as expected, which is consistent with the Doppler spread analysis. The VLC channel showed a larger coherence time than the values reported for a V2V-RF channel in the urban scenarios. We compared our results with other results given in literature as shown in Table. 2.3. Moreover, the V2V-VLC model showed that $2 \times 2$ MIMO-VLC links have much slower channel time variation compared to RF V2V links, and other VLC models.

- A VLC-based V2V system using the O-OFDM technique was investigated. Practical channel environment by employing various types of noise and reflectors was considered based on IEEE 802.15.7r1. An adaptive modulation scheme was used to fully utilize the transmission resources. The proposed model showed performance improvement (along with clipping distortion) for both the DCO-OFDM as well as the ACO-OFDM. A performance comparison between fixed and adaptive modulation schemes was performed and superiority in terms of BER and data rate was shown for the later. Moreover, we showed that a DCO-OFDM adaptive scheme has better data rate performance, while an ACO-OFDM scheme exhibits better BER characteristics. For instance, data rates of up to 50 Mbps can be achieved by using the DCO-OFDM scheme when the targeted BER is $10^{-5}$. Also, the simulation results showed that the number of assigned bits in the ACO-OFDM scheme is higher than in the DCO-OFDM scheme. Also, we studied 
the effect of the bias on the proposed system, where the simulation results showed that increasing the bias decreases the clipping noise and increases the number of bits assigned to each subcarrier.

- We investigated a precoder and equalization system for $2 \times 2 \mathrm{MIMO}-\mathrm{V} 2 \mathrm{~V}-\mathrm{VLC}$ system. We investigated a precoder and equalizer of three schemes, ZF, ML, and MMSE. We compared the investigated schemes performance in terms of BER and MSE. MMSE shows significant improvement comparing with ZF and ML precoders, where the BER is about $10^{-6}$ at $\mathrm{SNR}=15 \mathrm{~dB}$ and the normalized MSE is about $0.7 \times 10^{-2}$ when illuminance intensity $=1250 \mathrm{~cd}$.

- It is well known that the effect of shadowing on a VLC system is detrimental and can completely deteriorate a communication link. Hence, it is necessary to appropriately model shadowing effects as a preliminary step which was done in this work. From our studies, a two-edged obstructing object with uniform surface results in a bimodal distribution of the received power. With more severe and complicated shadowing, multi-modal reception appears. In this work, we proposed a method to take advantage of the optical diffraction phenomenon to overcome shadowing by employing a receiver with a wide FOV. We have modelled the shadowing effect for visible light by a bimodal distribution and derived the probability of error for no,i.e (absent), moderate and severe shadowing conditions. Simulation results showed the system could fail in the moderate and severe shadowing case where the BER is about $10^{-1}$ if a receiver with narrow FOV is used.

- In this work, we have proposed two denoising schemes to improve the performance of the V2V-VLC system under sunlight effect. We investigated the system performance for three scenarios: without optical filter, with optical filter, and with a proposed differential receiver. Simulation results showed that differential receiver can predict about $60 \%$ of solar irradiance and can improve the BER from $5 \times 10^{-4}$ up to $5 \times 10^{-5}$. 
Moreover, the results depicted V2V-VLC system can still work under solar irradiance where the worst achievable BER during the month of June is $\simeq 5 \times 10^{-4}$.

- Also, we have proposed a denoising scheme for the VLC-based V2V system by using MLE to mitigate the effect of solar irradiance on the transmitted optical signal. We considered a supervised learning scheme in MLE approaches. We proposed a kNN algorithm based adaptive filter to combat the effect of solar irradiance. The proposed system can adapt itself according to varying noise conditions and helps to achieve acceptable BER up to $10^{-4}$ for reliable communication. The proposed algorithm has been shown to exhibit numerical stability in dealing with a real data set. Also, simulation results showed good improvement in SNR, which made the communication link more stable. Moreover, simulation results showed that the optimal value of $\mathrm{K}$ is 12 with Euclidian distance method and $\mathrm{K}$ is 6 with City block distance method. Also, we discussed the execution time for the proposed scheme for the two distance methods. The results showed both methods have almost the same time of execution for a low numbers of data sets i.e., $0.05 s$ for 5000 data set, while City block method is better for a high number of data sets i.e., $0.32 s$ for 50000 data set.

- We proposed an infrastructure of roadside asset management by using Foglets and suggest a VLC link between smart vehicles and Foglets. This VLC link is high data rate point to point link without suffering from multipath fading and electromagnetic interference, thus improving upon efficiency and BER. If RF is used instead of the VLC link, the desired BER is not achieved due to multipath interference. We have presented the channel model between head/tail lights of vehicle and Foglet by considering multiple paths and environmental reflectors. We concluded that maximum optical power is received from direct path between vehicle and Foglet. This helps us to optimize the placement of Foglets in the proposed infrastructure.

From this work, we conclude that VLC is a promising candidate for next generations of indoor and outdoor wireless communication system. The advantages of VLC systems 
includes low cost equipment, no eavesdropping, unlicensed communication bandwidth, and low energy consumption. But due to the current technology, the communication data rate is limited to several Mbps. Obtained simulation's results confirm good performances and further emphasize the suitability of VLC technology for automobile applications.

\subsection{Future Developments and Perspectives}

VLC is an emerging technology with huge potential. Its suitability in the automotive field has been demonstrated with theoretical analyses and with simulation results. However, even if there are numerous traffic applications and situations in which VLC is well suited, it cannot be stated that VLC is able to support all the requirements and applications imposed in vehicular communications. In order to fully comply with the usage in vehicular communications, VLC still needs to enhance its performances. In the following some of the key issues that should be approached towards this goal.

\section{- Communication range}

One of the most important issues that should be improved is the communication range. In this case, the problem is that the power of the signal and consequently the SNR drops significantly when the communication range increases. Increasing the receiver gain is a suitable solution but still, it has its limits, meaning that additional studies should be taken. Nowadays the receiving element is based on a single photosensitive element used for the entire visible light spectrum, from 380 to $780 \mathrm{~nm}$. Its spectral sensitivity gets higher as the wavelength increases. This fact resulted in a shorter communication range. An efficient way to increase the communication range would be to design a receiver that uses an array of photo detectors, each of them dedicated to a specific wavelength (e.g. red, yellow, green). This way, the additional light corresponding to the other colors is filtered using an optical filter, leaving just the wavelength containing the data signal. The SNR level can be thus significantly enhanced and the communication range increased. 


\section{- Data rate improvement}

In indoor and at short distance VLC proved to be able of achieving very high data rates that can go up to $3 \mathrm{~Gb} / \mathrm{s}$. The high data rates are obtained using more complex modulations like OFDM or multi-level codes. In outdoor applications, the data rates are rarely above few tens of $\mathrm{Mb} / \mathrm{s}$. To improve this situation, future research should investigate the behavior of the indoor modulation techniques in the outdoor scenario.

\section{- Adaptability of the data rate}

In vehicular communications the packet delivery ratio is more important than the data rate. The delivery ratio is even more essential when high priority messages are involved. Nevertheless, the data rate is not negligible and a higher one is desirable. In these conditions, the coming work should focus on harmonizing between the two requirements, meaning that the data rate should be increased without affecting the packet delivery ratio. A possible solution would be the implementation of a communication protocol in which the data rate is adapted depending on the priority of the message. This way, the high priority messages can be sent at a low data rate to ensure the safe delivery, whereas the low priority messages can be sent at a higher data rate. Another interesting issue would be the development and the implementation of a duplex VLC system in which the two transceivers adapt the data rate based on the SNR level and on the imposed BER requirements. The VLC receivers can easily determine the noise level by analyzing the input signal when no data is received.

All these improvements can further increase the performances of VLC and ensure it a bright future. However, VLC still needs the support of the solid state lighting industry and of the photodiode industry as well. Future faster switching LEDs will enable higher data rates in indoor and outdoor applications, whereas more sensitive photo elements will enable longer distances. 


\section{Bibliography}

[1] [Online]. Available: http://www.solarelectricityhandbook.com/index.html.

[2] [Online]. Available: http://www.skyeninstruments.com.

[3] [Online]. Available: https://greenbusinesslight.com/resources/lighting.

[4] A.-M. Cilean, Study, implementation and optimization of a visible light communications system. Application to automotive field. PhD Thesis, 2014.

[5] N. Kumar, Visible light communication systems for road safety applications. PhD Thesis, 2011.

[6] [Online]. Available: http://en.wikipedia.org/wiki/Automotive_lighting

[7] P. Luo, Z. Ghassemlooy, H. L. Minh, E. Bentley, A. Burton, and X. Tang, "Fundamental analysis of a car to car visible light communication system," in Communication Systems, Networks Digital Signal Processing (CSNDSP), 2014 9th International Symposium on, July 2014, pp. 1011-1016.

[8] [Online]. Available: http://www.shadesdaddyblog.com/are-all-sunglasses-uv-protected.

[9] H. E. R. Mesleh and H. Haas, "On the performance of different OFDM based optical wireless communication systems," IEEE/OSA Journal of Optical Communications and Networking, vol. 3, no. 8, pp. 620-628, August 2011. 
[10] D. Terra, N. Kumar, N. Loureno, L. N. Alves, and R. L. Aguiar, "Design, development and performance analysis of DSSS-based transceiver for VLC," in 2011 IEEE EUROCON - International Conference on Computer as a Tool, April 2011, pp. 1-4.

[11] H. L. Minh, D. O'Brien, G. Faulkner, L. Zeng, K. Lee, D. Jung, Y. Oh, and E. T. Won, "100-Mb/s NRZ Visible Light Communications Using a Postequalized White LED," IEEE Photonics Technology Letters, vol. 21, no. 15, pp. 1063-1065, Aug 2009.

[12] S. Hranilovic, "Wireless Optical Communication Systems," in New York : Springer, 2005., Jan 2005, ISBN 9781441919823.

[13] I. A. Mahmoud Beshr and M. Hussien, "The impact of sunlight on the performance of visible light communication systems over the year," in Proceedings of SPIE-The International Society for Optical Engineering, 2012.

[14] Shun-Hsiang Yu , , Shih, O., Hsin-Mu Tsai , Roberts, R, "Smart automotive lighting for vehicle safety," IEEE Communications Magazine, vol. 12, no. 51, pp. 50-59, Feb 2013.

[15] T. Komine and M. Nakagawa, "Fundamental analysis for visible-light communication system using LED lights," IEEE Transactions of Consumer Electronics, vol. 50, no. 1, pp. 100-107, Feb 2004.

[16] SeokJu Lee; Jae Kyun Kwon; Sung-Yoon Jung ; Young-Hoon Kwon., "Evaluation of visible light communication channel delay profiles for automotive applications," EURASIP Journal on wireless communications and networkin, pp. 1823-1826, 2012.

[17] J. P. Peyton Z., Probability, Random Variables, and Random Signal Principles. McGraw-Hill Book Co, Fourth Edition.

[18] G. P. AGRAWAL, Fiber-Optic Communication Systems. John Wiley and Sons, 2012, Fourth Edition. 
[19] P. vanRooyen and F. Solms, "Maximum entropy investigation of the inter user interference distribution in a DS/SSMA system," in Proceedings of 6th International Symposium on Personal, Indoor and Mobile Radio Communications, vol. 3, Sept 1995.

[20] Q. f. Lu, X. s. Ji, and K. z. Huang, "Clipping distortion analysis and optimal power allocation for ACO-OFDM based visible light communication," in 2014 4th IEEE International Conference on Information Science and Technology, April 2014, pp. $320-323$.

[21] [Online]. Available: http://www.hamamatsu.com/resources/ssd/s8664

[22] [Online]. Available: http://www.who.int/news-room/fact-sheets/detail.

[23] P. Papadimitratos, A. D. L. Fortelle, K. Evenssen, R. Brignolo, and S. Cosenza, "Vehicular communication systems: Enabling technologies, applications, and future outlook on intelligent transportation," IEEE Communications Magazine, vol. 47, no. 11, pp. 84-95, November 2009.

[24] A. Agarwal and T. D. C. Little, "Role of directional wireless communication in vehicular networks," in 2010 IEEE Intelligent Vehicles Symposium, June 2010, pp. 688-693.

[25] T. D. C. Little, A. Agarwal, J. Chau, M. Figueroa, A. Ganick, J. Lobo, T. Rich, and P. Schimitsch, "Directional communication system for short-range vehicular communications," in 2010 IEEE Vehicular Networking Conference, Dec 2010, pp. 231-238.

[26] B. K. E. W. Liu, C.; Sadeghi, "Enabling vehicular visible light communication (V2LC) networks," in Proceedings of the Eighth ACM international workshop on Vehicular inter-networking (VANET '11), ACM, New York, NY, USA, 2011, pp. 41-50.

[27] C. C. L. H. K. T. Pang, G.K.H., "Dual use of LEDs : Signaling and communications in ITS," in Proceedings of fifth World Congress on Intelligent Transport Systems, Paper 3035, Seoul, Korea, 1998, pp. 12-16. 
[28] G. Hugh Sing Liu; Pang, "Positioning beacon system using digital camera and LEDs," Vehicular Technology, IEEE Transactions, vol. 52, no. 2, pp. 406-419, March 2003.

[29] M. Wada, T. Yendo, T. Fujii, and M. Tanimoto, "Road-to-vehicle communication using LED traffic light," in IEEE Proceedings. Intelligent Vehicles Symposium, 2005., June 2005, pp. 601-606.

[30] T. Hara, S. Iwasaki, T. Yendo, T. Fujii, and M. Tanimoto, "A new receiving system of visible light communication for ITS," in 2007 IEEE Intelligent Vehicles Symposium, June 2007, pp. 474-479.

[31] T. Nagura, T. Yamazato, M. Katayama, T. Yendo, T. Fujii, and H. Okada, "Improved decoding methods of visible light communication system for ITS using LED array and high-speed camera," in 2010 IEEE 71st Vehicular Technology Conference, May 2010, pp. $1-5$.

[32] I. Takai, S. Ito, K. Yasutomi, K. Kagawa, M. Andoh, and S. Kawahito, "LED and CMOS image sensor based optical wireless communication system for automotive applications," IEEE Photonics Journal, vol. 5, no. 5, pp. 6801418-6801418, Oct 2013.

[33] T. Yamazato, I. Takai, H. Okada, T. Fujii, T. Yendo, S. Arai, M. Andoh, T. Harada, K. Yasutomi, K. Kagawa, and S. Kawahito, "Image-sensor-based visible light communication for automotive applications," IEEE Communications Magazine, vol. 52, no. 7, pp. 88-97, July 2014.

[34] N. Kumar, N. Loureno, D. Terra, L. N. Alves, and R. L. Aguiar, "Visible light communications in intelligent transportation systems," in 2012 IEEE Intelligent Vehicles Symposium, June 2012, pp. 748-753.

[35] D. C. O'Brien, L. Zeng, H. Le-Minh, G. Faulkner, J. W. Walewski, and S. Randel, "Visible light communications: Challenges and possibilities," in 2008 IEEE 19th 
International Symposium on Personal, Indoor and Mobile Radio Communications, Sept 2008, pp. 1-5.

[36] J. Hou and D. C. O’Brien., "Vertical handover-decision-making algorithm using fuzzy logic for the integrated Radio-and-OW system," IEEE Transactions on Wireless Communications, 2006.

[37] OMEGA, "Omega project," in available online :www.ict-omega.eu.

[38] S. Rajagopal, R. D. Roberts, and S. K. Lim, "IEEE 802.15.7 visible light communication: modulation schemes and dimming support," IEEE Communications Magazine, vol. 50, no. 3, pp. 72-82, March 2012.

[39] M. Uysal, Z. Ghassemlooy, A. Bekkali, A. Kadri, and H. Menouar, "Visible light communication for vehicular networking: Performance study of a V2V system using a measured headlamp beam pattern model," IEEE Vehicular Technology Magazine, vol. 10, no. 4, pp. 45-53, Dec 2015.

[40] P. L. et.al., "Performance analysis of a car-to-car visible light communication system," Applied Optics, vol. 54, no. 7, p. 16961706, Feb 2015.

[41] I. Lee, M. Sim, and F. Kung, "A dual-receiving visible-light communication system under time-variant non-clear sky channel for intelligent transportation system," in Networks and Optical Communications (NOC), 2011 16th European Conference on, July 2011, pp. 153-156.

[42] Z. Cui, C. Wang, and H.-M. Tsai, "Characterizing channel fading in vehicular visible light communications with video data," in Vehicular Networking Conference (VNC), 2014 IEEE, Dec 2014, pp. 226-229.

[43] S.-Y. J. SeokJu Lee, Jae Kyun Kwon and Y.-H. Kwon., "Evaluation of visible light communication channel delay profiles for automotive applications," EURASIP Journal on wireless communications and networking, vol. 1, pp. 370-379, Dec 2012. 
[44] N. Araki and H. Yashima, "A channel model of optical wireless communications during rainfall," in Wireless Communication Systems, 2005. 2nd International Symposium on, Sept 2005, pp. 205-209.

[45] S. Lee, J. Kwon, S. Jung, and Y. Kwon, "Simulation modeling of visible light communication channel for automotive applications," in Intelligent Transportation Systems (ITSC), 2012 15th International IEEE Conference on, Sept 2012, pp. 463-468.

[46] M. Kinoshita, T. Yamazato, H. Okada, T. Fujii, S. Arai, T. Yendo, and K. Kamakura, "Motion modeling of mobile transmitter for image sensor based I2V-VLC, V2I-VLC, and V2V-VLC," in Globecom Workshops (GC Wkshps), 2014, Dec 2014, pp. 450-455.

[47] S. K. M. Sivak, M. J. Flannagan and E. C. Traube, "A market weighted description of low-beam headlighting patterns in the us," Proceeding of the IEEE, vol. 1, no. 4, pp. $61-71,2005$.

[48] [Online]. Available: http://en.wikipedia.org/wiki/Headlamp

[49] J. P. C. Q. Geng, "Results of a new polarimetric BRDF simulation of metallic surfaces," Infrared Physics and Technology, 2015.

[50] [Online]. Available: www.mto.gov.on.ca/english/dandv/driver/handbook

[51] H. Schulze, "Frequency-domain simulation of the indoor wireless optical communication channel," IEEE Transactions on Communications, vol. 64, no. 6, pp. 2551-2562, June 2016.

[52] J. B. J.M. Kahn, "Wireless infrared communications," Proceeding of the IEEE, vol. 85, no. 2, pp. 265-298, Feb 1997.

[53] K. L. Changping Li, Ying Yi and K. Lee, "Performance analysis of visible light communication using the STBC-OFDM technique for intelligent transportation systems," International Journal of Electronics, vol. 101, no. 8, pp. 1117-1133, August 2014. 
[54] C. Wang, H. Y. Yu, and Y. J. Zhu, "A long distance underwater visible light communication system with single photon avalanche diode," IEEE Photonics Journal, vol. 8, no. 5, pp. 1-11, Oct 2016.

[55] T.-H. Do and M. Yoo, "Received power and SNR optimization for visible light communication system," in Fourth International Conference on Ubiquitous and Future Networks (ICUFN), 2012.

[56] E. Sarbazi, M. Uysal, M. Abdallah, and K. Qaraqe, "Ray tracing based channel modeling for visible light communications," in 2014 22nd Signal Processing and Communications Applications Conference (SIU), April 2014, pp. 702-705.

[57] F. Miramirkhani and M. Uysal, "Channel modeling and characterization for visible light communications," IEEE Photonics Journal, vol. 7, no. 6, pp. 1-16, Dec 2015.

[58] M. S. Varela and M. G. Sanchez, "RMS delay and coherence bandwidth measurements in indoor radio channels in the UHF band," IEEE Trans. Veh. Technol., vol. 50, no. 2, pp. 515-525, Mar 2001.

[59] [Online]. Available: http://www.powerbulbs.com/us/product/philips-x.

[60] J. B. Carruthers and J. M. Kahn, "Modeling of nondirected wireless infrared channels," IEEE Transactions on Communications, vol. 45, no. 10, pp. 1260-1268, Oct 1997.

[61] C. F. Mecklenbrauker, A. F. Molisch, J. Karedal, F. Tufvesson, A. Paier, L. Bernado, T. Zemen, O. Klemp, and N. Czink, "Vehicular channel characterization and its implications for wireless system design and performance," Proceedings of the IEEE, vol. 99, no. 7, pp. 1189-1212, July 2011.

[62] L. Cheng, B. E. Henty, D. D. Stancil, F. Bai, and P. Mudalige, "Mobile vehicle-to-vehicle narrow-band channel measurement and characterization of the $5.9 \mathrm{GHz}$ dedicated short range communication DSRC frequency band," IEEE Journal on Selected Areas in Communications, vol. 25, no. 8, pp. 1501-1516, Oct 2007. 
[63] A. L. Chen, H. P. Wu, Y. L. Wei, and H. M. Tsai, "Time variation in vehicle-to-vehicle visible light communication channels," in 2016 IEEE Vehicular Networking Conference (VNC), Dec 2016, pp. 1-8.

[64] X. Zhang, Y. F. Zhou, Y. P. Yu, P. C. Han, and X. R. Wang, "Comparison and analysis of DCO-OFDM, ACO-OFDM and ADO-OFDM in IM/DD systems," Applied Mechanics and Materials, vol. 701-702, pp. 1059-1062, 122014.

[65] Q. Wang, Z. Wang, and L. Dai, "Multiuser MIMO-OFDM for visible light communications," IEEE Photonics Journal, vol. 7, no. 6, pp. 1-11, 2015.

[66] J. D. Liang Wu, Zaichen Zhang and H. Liu, "Adaptive modulation schemes for visible light communications," Journal of Lightwave Technology, 2015.

[67] Y. Goto, I. Takai, T. Yamazato, H. Okada, T. Fujii, S. Kawahito, S. Arai, T. Yendo, and K. Kamakura, "A new automotive VLC system using optical communication image sensor," IEEE Photonics Journal, vol. 8, no. 3, pp. 1-17, June 2016.

[68] A.-M. Cailean and M. Dimian., "Toward environmental-adaptive visible light communications receivers for automotive applications: A review." IEEE Sensors Journal, vol. 16, no. 9, 2016.

[69] B. Turan, O. Narmanlioglu, S. C. Ergen, and M. Uysal, "Broadcasting brake lights with MIMO-OFDM based vehicular VLC," in 2016 IEEE Vehicular Networking Conference (VNC), Dec 2016, pp. 1-7.

[70] Y. H. a. V. E. Fernando, N., "Flip-OFDM for unipolar communication systems," IEEE Transactions on Communications, 2012.

[71] G. T. Z. Zhenhua Yu, Robert J. Baxley, "EVM and achievable data rate analysis of clipped OFDM signals in visible light communication," EURASIP Journal on Wireless Communications and Networking, 2012. 
[72] D. Z. Xintong LingJiaheng, WangXiao LiangZhi, "Offset and power optimization for DCO-OFDM in visible light communication systems," IEEE Transactions on Signal Processing, vol. 64, no. 2, pp. 349-363, Jan 2016.

[73] J. D. Liang Wu, Zaichen Zhang and H. Liu, "Adaptive modulation schemes for visible light communications," Journal of Lightwave Technology, vol. 33, no. 1, pp. 117-125, January 2015.

[74] D. P. Palomar, J. M. Cioffi, and M. A. Lagunas, "Joint Tx-Rx beamforming design for multicarrier MIMO channels: a unified framework for convex optimization," IEEE Transactions on Signal Processing, vol. 51, no. 9, pp. 2381-2401, Sept 2003.

[75] R. Wang, Q. Gao, J. You, E. Liu, P. Wang, Z. Xu, and Y. Hua, "Linear transceiver designs for MIMO indoor visible light communications under lighting constraints," IEEE Transactions on Communications, vol. 65, no. 6, pp. 2494-2508, 2017.

[76] H. Elgala, R. Mesleh, and H. Haas, "An LED model for intensity-modulated optical communication systems," IEEE Photonics Technology Letters, vol. 22, no. 11, pp. 835-837, June 2010.

[77] B. Toma, H. M. Tsai, and M. Boban, "Simulating vehicular visible light communication: Physical radio and MAC modeling," in 2014 IEEE Vehicular Networking Conference (VNC), Dec 2014, pp. 222-225.

[78] J. Y. E. L. P. W. Z. X. Rui Wang, Qian Gao and Y. Hua., "Linear transceiver designs for MIMO indoor visible light communications under lighting constraints." IEEE Transactions on Communications, vol. 65, no. 6, 2017.

[79] L. Zeng, D. O'brien, H. Minh, G. Faulkner, K. Lee, D. Jung, Y. Oh, and E. T. Won, "High data rate multiple input multiple output (MIMO) optical wireless communications using white LED lighting," Selected Areas in Communications, vol. 27, no. 9, pp. 1654-1662, December 2009. 
[80] Y.-C. K. Ki-Hong Park and A. M, "On the power and offset allocation for rate adaptation of spatial multiplexing in optical wireless MIMO channels," IEEE Transactions on Communications, vol. 61, no. 4, pp. 1535-1543, 2013.

[81] Q. H.-B. R. J. Ying, K. and S. Yao, "Joint optimization of precoder and equalizer in MIMO VLC systems." IEEE Journal on Selected Areas in Communications, vol. 33, no. $9,2015$.

[82] H. Ma, L. Lampe, and S. Hranilovic, "Robust MMSE linear precoding for visible light communication broadcasting systems," in 2013 IEEE Globecom Workshops (GC Wkshps), Dec 2013, pp. 1081-1086.

[83] Y.-C. K. Ki-Hong Park and M.-S. Alouini, "On the power and offset allocation for rate adaptation of spatial multiplexing in optical wireless MIMO channels," IEEE Transactions on Communications, vol. 61, no. 4, pp. 1535-1543, 2013.

[84] H. E. P. M. Butala and T. D. C. Little, "SVD-VLC: A novel capacity maximizing VLC MIMO system architecture under illumination constraints," in in Proc. IEEE Globecom Workshop OWC, 2013.

[85] Z.-Q. L. Yanwu Ding, T.N. Davidson and K. M. Wong, "Minimum BER block precoders for zero-forcing equalization," IEEE Transactions on Signal Processing, vol. 51, no. 9, pp. 2410-2423, Sept 2003.

[86] T. Ozaki, H. Iwai, and H. Sasaoka, "Improvement of transmission performance by variable precoding in MIMO transmission," IEICE Communications Express, vol. 3, no. 5, pp. 168-174, 2014.

[87] T. N. D. Scarlett S. Chan and K. M. Wong, "Asymptotically minimum BER linear block precoders for mmse equalisation," in Communications, IEE Proceedings, vol. 151, no. 4, 2004, pp. 297-304. 
[88] D. FANG, Optimal Precoder Design and Block-Equal QRS Decomposition for ML Based Successive Cancellation Detection. Master Thesis, 2013.

[89] Hasan Farahneh, Sikder Kamruzzaman, and Xavier Fernando, "Differential receiver as a denoising scheme to improve the performance of V2V-VLC systems," in $I C C$ 2018-USA, 2018.

[90] B. E. S. B. J. Zhang, Chidong; Mapes, "Bimodality in tropical water vapour," Quarterly Journal of the Royal Meteorological Society, vol. 129, no. 594, pp. 2847-2866, Oct 2003.

[91] N. H. B. P. Catherine Forbes, Merran Evans, "Statistical Distributions,4th Edition," in New York :Jhon Willy, 2005., 2005,ISBN 978-0470390634.

[92] J. B. Carruthers and J. M. Kahn, "Modeling of nondirected wireless infrared channels," IEEE Transaction on Communication, vol. 45, December 1997.

[93] F. Gfeller and U. Bapst., "Wireless in-house data communication via diffuse infrared radiation," in in Proc.IEEE, vol. 67, Nov 1979, pp. 1474-1486.

[94] J. A. Duffie and W. A. Beckman, Solar Engineering of Thermal Processes. Wiley, 2013.

[95] I. E. Lee, M. L. Sim, and F. W. L. Kung, "Performance enhancement of outdoor visible-light communication system using selective combining receiver," IET Optoelectronics, vol. 3, no. 1, pp. 30-39, Feb 2009.

[96] M. S. Islim and H. Haas, "An investigation of the sunlight irradiance effect on visible light communications," 2017.

[97] M. F. F. Khan, A. Akif, and M. A. Haque, "Iris recognition using machine learning from smartphone captured images in visible light," in 2017 IEEE International Conference on Telecommunications and Photonics (ICTP), Dec 2017, pp. 33-37. 
[98] Wei Sun, Chunyu, Zhao, Long Chen, Dajian Li, Yicheng Bai, Wenyan Jia, Mingui Sun, "Learning based particle filtering object tracking for visible-light systems," International Journal for Light and Electron Optics, vol. 126, no. 9, pp. 1830-1837, 2015.

[99] Xiansheng Guo, Sihua Shao, Nirwan Ansari, Abdallah Khreishah, "Indoor localization using visible light via fusion of multiple classiers," IEEE photonics journal, vol. 9, no. 6, 2017.

[100] G. Keiser, Optical Communications Essentials. McGraw-Hill, 2003.

[101] Y. H. Chung and S. b. Oh, "Efficient optical filtering for outdoor visible light communications in the presence of sunlight or articifical light," in 2013 International Symposium on Intelligent Signal Processing and Communication Systems, Nov 2013, pp. 749-752.

[102] M. Khaligh, F. Xu, Y. Jaafar, and S. Bourennane, "Double-laser differential signaling for reducing the effect of background radiation in free-space optical systems," IEEE/OSA Journal of Optical Communications and Networking, vol. 3, no. 2, pp. 145-154, Feb 2011.

[103] N. Karaboga, "Digital IIR filter design using differential evolution algorithm," EURASIP Journal on Advances in Signal Processing, vol. 2005, no. 8, pp. 1269-1276, May 2005.

[104] M. Z. W. W. J. H. Z. J. C. H. Deng Zhen, Jiang Yang, "A novel wavelength-adjusting method in ingan-based light-emitting diodes," Scientific Reports.

[105] A. Al-Fuqaha and et al., "Internet of Things: A Survey on Enabling Technologies, Protocols, and Applications," IEEE Communications Surveys and Tutorials, vol. 17, no. 4, pp. 2347-2376, November 2015.

[106] I. Stojmenovic, "Fog computing: A cloud to the ground support for smart things and machine-to-machine networks," IEEE Conference on Australasian Telecommunication Networks and Applications, pp. 117-122, November 2014. 
[107] Hung.et.al, "Architecture Harmonization Between Cloud Radio Access Networks and Fog Networks," IEEE Translations on Emerging Cloud-Based Wireless Communications and Networks, vol. 3, no. 5, pp. 3013-3034, January 2015.

[108] F. Bonomi and et al., "Fog computing: A platform for internet of things and analytics," Springer; A Roadmap for Smart Environments, Studies in Computational Intelligence 546, pp. 169-186, 2015.

[109] W. Viriyasitavat and et al., "Short paper: Channel model for visible light communications using off-the-shelf scooter taillight," in 2013 IEEE Vehicular Networking Conference, Dec 2013, pp. 170-173.

[110] I. Takai and M. Andoh, "Optical vehicle-to-vehicle communication system using LED transmitter and camera receiver," IEEE Photonic Journal, vol. 6, no. 5, pp. 1-14, October 2014.

[111] C. Hongsong and Z. Dongyan, "Security and Trust Research in M2M System," IEEE Vehicular Technology Conference, pp. 286-290, Janauary 2011. 